\title{
Floer homology of cotangent bundles and the loop product
}

\author{
Alberto ABBondandolo \\ MATTHIAS SCHWARZ
}

\begin{abstract}
We prove that the pair-of-pants product on the Floer homology of the cotangent bundle of a compact manifold $M$ corresponds to the Chas-Sullivan loop product on the singular homology of the loop space of $M$. We also prove related results concerning the Floer homological interpretation of the Pontrjagin product and of the Serre fibration. The techniques include a Fredholm theory for Cauchy-Riemann operators with jumping Lagrangian boundary conditions of conormal type, and a new cobordism argument replacing the standard gluing technique.
\end{abstract}

53D40, 57R58; 55N45

\section{Introduction}

Let $M$ be a closed manifold, and let $H$ be a time-dependent smooth Hamiltonian on $T^{*} M$, the cotangent bundle of $M$. We assume that $H$ is 1 -periodic in time and grows asymptotically quadratically on each fiber. Generically, the corresponding Hamiltonian system

$$
x^{\prime}(t)=X_{H}(t, x(t))
$$

has a discrete set $\mathscr{P}(H)$ of 1 -periodic orbits. The free abelian group $F_{*}(H)$ generated by the elements in $\mathscr{P}(H)$, graded by their Conley-Zehnder index, supports a chain complex, the Floer complex $\left(F_{*}(H), \partial\right)$. The boundary operator $\partial$ is defined by an algebraic count of the maps $u$ from the cylinder $\mathbb{R} \times \mathbb{T}$ to $T^{*} M$, solving the Cauchy-Riemann type equation

$$
\partial_{s} u(s, t)+J(u(s, t))\left(\partial_{t} u(s, t)-X_{H}(t, u(s, t))\right)=0, \quad \forall(s, t) \in \mathbb{R} \times \mathbb{T},
$$

and converging to two 1 -periodic orbits of (0-1) for $s \rightarrow-\infty$ and $s \rightarrow+\infty$. Here $J$ is the almost-complex structure on $T^{*} M$ induced by a Riemannian metric on $M$, and (0-2) can be seen as the negative $L^{2}$-gradient equation for the Hamiltonian action functional.

This construction is due to A Floer (eg $[21 ; 22 ; 23 ; 24])$ in the case of a closed symplectic manifold $P$, in order to prove a conjecture of Arnold on the number of 
periodic Hamiltonian orbits. The extension to noncompact symplectic manifolds, such as the cotangent bundles we consider here, requires suitable growth conditions on the Hamiltonian, such as the convexity assumption used by C Viterbo [52] or the asymptotic quadratic-growth assumption used by the authors [4]. The Floer complex obviously depends on the Hamiltonian $H$, but its homology often does not, so it makes sense to call this homology the Floer homology of the underlying symplectic manifold $P$, and to denote it by $H F_{*}(P)$. The Floer homology of a compact symplectic manifold $P$ without boundary is isomorphic to the singular homology of $P$, as proved by A Floer for special classes of symplectic manifolds, and later extended to larger and larger classes by several authors (the general case requiring special coefficient rings; see Hofer and Salamon [31], Liu and Tian [36] and Fukaya and Ono [30]). Unlike the compact case, the Floer homology of a cotangent bundle $T^{*} M$ is a truly infinite dimensional homology theory, being isomorphic to the singular homology of the free loop space $\Lambda(M)$ of $M$. This fact was first proved by C Viterbo [52] using a generating functions approach, later by D Salamon and J Weber [44] using the heat flow for curves on a Riemannian manifold, and then by the authors in [4]. In particular, our proof reduces the general case to the case of a Hamiltonian which is uniformly convex in the momenta, and for such a Hamiltonian it constructs an explicit isomorphism between the Floer complex of $H$ and the Morse complex of the action functional

$$
\mathbb{S}_{L}(\gamma)=\int_{\mathbb{T}} L\left(t, \gamma(t), \gamma^{\prime}(t)\right) d t, \quad \gamma \in W^{1,2}(\mathbb{T}, M),
$$

associated to the Lagrangian $L$ which is the Fenchel dual of $H$. The latter complex is the standard chain complex associated to the Lagrangian action functional $\mathbb{S}_{L}$. The domain of such a functional is the infinite dimensional Hilbert manifold $W^{1,2}(\mathbb{T}, M)$ consisting of closed loops of Sobolev class $W^{1,2}$ on $M$, and an important fact is that the functional $\mathbb{S}_{L}$ is bounded from below, has critical points with finite Morse index, satisfies the Palais-Smale condition, and, although in general it is not $C^{2}$, it admits a smooth Morse-Smale pseudo-gradient flow. The construction of the Morse complex in this infinite dimensional setting and the proof that its homology is isomorphic to the singular homology of the ambient manifold are described by the first author and Majer [1]. The isomorphism between the Floer and the Morse complex is obtained by coupling the Cauchy-Riemann type equation on half-cylinders with the gradient flow equation for the Lagrangian action. We call this the hybrid method.

Since the space $W^{1,2}(\mathbb{T}, M)$ is homotopy equivalent to $\Lambda(M)$, we get the required isomorphism

$$
\Phi^{\Lambda}: H_{*}(\Lambda(M)) \stackrel{\cong}{\longrightarrow} H F_{*}\left(T^{*} M\right)
$$


from the singular homology of the free loop space of $M$ to the Floer homology of $T^{*} M$.

Additional interesting algebraic structures on the Floer homology of a symplectic manifold are obtained by considering other Riemann surfaces than the cylinder as domain for the Cauchy-Riemann type equation (0-2). By considering the pair-of-pants surface, a noncompact Riemann surface with three cylindrical ends, one obtains the pairof-pants product in Floer homology (see the second author's thesis [46] and McDuff and Salamon [38]). When the symplectic manifold $P$ is closed and symplectically aspherical, this product corresponds to the standard cup product from topology, after identifying the Floer homology of $P$ with its singular cohomology by Poincaré duality, while when the manifold $P$ can carry $J$-holomorphic spheres, the pair-of-pants product corresponds to the quantum cup product of $P$ (see Piunikhin, Salamon and Schwarz [39] and Liu and Tian [37]).

The main result of this paper is that in the case of cotangent bundles, the pair-of-pants product is also equivalent to a product on $H_{*}(\Lambda(M))$ coming from topology, but a more interesting one than the simple cup product:

Theorem A Let $M$ be a closed oriented manifold. Then the isomorphism $\Phi^{\Lambda}$ in (0-3) is a ring isomorphism when the Floer homology of $T^{*} M$ is endowed with its pair-of-pants product, and the homology of the space of free parametrized loops of $M$ is endowed with its Chas-Sullivan loop product.

The latter is an algebraic structure which was recently discovered by $\mathrm{M}$ Chas and D Sullivan [9], and which is currently having a strong impact in string topology (see eg Cohen, Hess and Voronov [14] and Sullivan [50]). It is the free loop space version of the classical Pontrjagin product

$$
\text { \# : } H_{j}\left(\Omega\left(M, q_{0}\right)\right) \otimes H_{k}\left(\Omega\left(M, q_{0}\right)\right) \rightarrow H_{j+k}\left(\Omega\left(M, q_{0}\right)\right)
$$

on the singular homology of the space $\Omega\left(M, q_{0}\right)$ of loops based at $q_{0}$. As the Pontrjagin product, it is induced by concatenation and it can be described in the following way. Let $\Theta(M)$ be the subspace of $\Lambda(M) \times \Lambda(M)$ consisting of pairs of parametrized loops with identical initial point. If $M$ is oriented and $n$-dimensional, $\Theta(M)$ is both a co-oriented $n$-codimensional submanifold of the Banach manifold $\Lambda(M) \times \Lambda(M)$, as well as of $\Lambda(M)$ itself via the concatenation map $\Gamma: \Theta(M) \rightarrow \Lambda(M)$ :

$$
\Lambda(M) \times \Lambda(M) \stackrel{e}{\hookleftarrow} \Theta(M) \stackrel{\Gamma}{\hookrightarrow} \Lambda(M) .
$$


Seen as continuous maps, $e$ and $\Gamma$ induce homomorphisms $e_{*}, \Gamma_{*}$ in homology. Seen as $n$-codimensional co-oriented embeddings, they induce Umkehr maps

$$
\begin{aligned}
e_{!}: H_{j}(\Lambda(M) \times \Lambda(M)) & \rightarrow H_{j-n}(\Theta(M)), \\
\Gamma_{!}: H_{j}(\Lambda(M)) & \rightarrow H_{j-n}(\Theta(M)) .
\end{aligned}
$$

The loop product is the degree $-n$ product on the homology of the free loop space of $M$,

$$
\circ: H_{j}(\Lambda(M)) \otimes H_{k}(\Lambda(M)) \rightarrow H_{j+k-n}(\Lambda(M)),
$$

defined as the composition

$$
\begin{aligned}
H_{j}(\Lambda(M)) \otimes H_{k}(\Lambda(M)) \stackrel{\times}{\longrightarrow} H_{j+k}(\Lambda(M) \times \Lambda(M)) \stackrel{e_{!}}{\longrightarrow} H_{j+k-n}(\Theta(M)) \\
\stackrel{\Gamma_{*}}{\longrightarrow} H_{j+k-n}(\Lambda(M)),
\end{aligned}
$$

where $x$ is the exterior homology product. The loop product turns out to be associative, commutative, and to have a unit, namely the image of the fundamental class of $M$ by the embedding of $M$ into $\Lambda(M)$ as the space of constant loops. More information about the loop product and about its relationship with the Pontrjagin and the intersection product on $M$ are recalled in Section 1. Similarly, the composition $e_{*} \circ \Gamma_{\text {! gives a }}$ coproduct of degree $-n$ (for coefficients in a field), corresponding to the pair-of-pants coproduct on Floer homology. However, it is easy to see that this coproduct is almost entirely trivial, except for homology classes of dimension $n$, so we shall not consider it in this paper.

Coming back to Theorem A, it is worth noticing that the analogy between the pair-ofpants product and the loop product is even deeper. Indeed, we may look at the solutions $\left(x_{1}, x_{2}\right):[0,1] \rightarrow T^{*} M \times T^{*} M$ of the following pair of Hamiltonian systems

$$
x_{1}^{\prime}(t)=X_{H_{1}}\left(t, x_{1}(t)\right), \quad x_{2}^{\prime}(t)=X_{H_{2}}\left(t, x_{1}(t)\right),
$$

coupled by the nonlocal boundary condition

$$
\begin{aligned}
& q_{1}(0)=q_{1}(1)=q_{2}(0)=q_{2}(1), \\
& p_{1}(1)-p_{1}(0)=p_{2}(0)-p_{2}(1) .
\end{aligned}
$$

Here we are using the notation $x_{j}(t)=\left(q_{j}(t), p_{j}(t)\right)$, with $q_{j}(t) \in M$ and $p_{j}(t) \in$ $T_{q_{j}(t)}^{*} M$, for $j=1,2$. By studying the corresponding Lagrangian boundary value Cauchy-Riemann type problem on the strip $\mathbb{R} \times[0,1]$, we obtain a chain complex, the Floer complex for figure-8 loops $\left(F^{\Theta}\left(H_{1} \oplus H_{2}\right)\right.$, $\left.\partial\right)$ on the graded free abelian group generated by solutions of (0-5)-(0-6). Then we can show that:

(i) The homology of the chain complex $\left(F^{\Theta}\left(H_{1} \oplus H_{2}\right)\right.$, $\left.\partial\right)$ is isomorphic to the singular homology of $\Theta(M)$. 
(ii) The pair of pants product factors through the homology of this chain complex.

(iii) The first homomorphism in this factorization corresponds to the homomorphism $e_{!} \circ \times$, while the second one corresponds to the homomorphism $\Gamma_{*}$.

We also show that similar results hold for the space $\Omega\left(M, q_{0}\right)$ of loops which are based at $q_{0} \in M$. The Hamiltonian problem in this case is Equation (0-1) for $x=$ $(q, p):[0,1] \rightarrow T^{*} M$ with boundary conditions

$$
q(0)=q(1)=q_{0} .
$$

Since the fiber $T_{q_{0}}^{*} M$ is a Lagrangian submanifold of $T^{*} M$, this is a Lagrangian intersection problem, and one can associate to it a Floer homology, that we denote by $H F_{*}^{\Omega}\left(T^{*} M\right)$. On such a Floer homology there is a product

$$
\Upsilon_{*}^{\Omega}: H F_{j}^{\Omega}\left(T^{*} M\right) \otimes H F_{k}^{\Omega}\left(T^{*} M\right) \rightarrow H F_{j+k}^{\Omega}\left(T^{*} M\right),
$$

which is called the triangle product. Then we can prove the following:

Theorem B Let $M$ be a closed manifold. Then there is a ring isomorphism

$$
\Phi^{\Omega}: H_{*}\left(\Omega\left(M, q_{0}\right)\right) \stackrel{\cong}{\longrightarrow} H F_{*}^{\Omega}\left(T^{*} M\right),
$$

where the singular homology of the based loop space $\Omega\left(M, q_{0}\right)$ is endowed with the Pontrjagin product \# and the Floer homology $H F_{*}^{\Omega}\left(T^{*} M\right)$ is equipped with the triangle product.

Actually, every arrow in the commutative diagram from topology

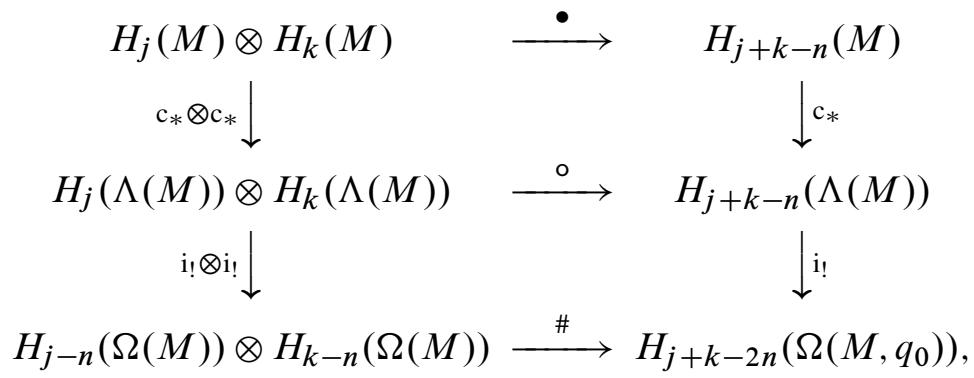

has an equivalent homomorphism in Floer homology. Here $\bullet$ is the intersection product in singular homology, $\mathrm{c}$ is the embedding of $M$ into $\Lambda(M)$ by constant loops, and i! denotes the Umkehr map induced by the $n$-codimensional co-oriented embedding i: $\Omega(M) \hookrightarrow \Lambda(M)$.

The first step in the proof of the main statements of this paper is to describe objects and morphisms from algebraic topology in a Morse theoretical way. The way this 
translation is performed is well known in the case of finite dimensional manifolds (see eg Fukaya [27], Schwarz [45], Betz and Cohen [7], Viterbo [51] and Fukaya [28]). In the Appendix we outline how these results extend to infinite dimensional Hilbert manifolds, paying particular attention to the transversality conditions required for each construction. In Section 2, we specialize the analysis to the action functional associated to Lagrangians which have quadratic growth in the velocities. See also Cohen [13], Cohen, Hess and Voronov [14] and Cohen and Schwarz [18].

The core of the paper consists of Section 3 and Section 4. In the former we define the Floer complexes we are dealing with and the products on their homology. All the Floer homologies we consider here - for free loops, based loops or figure-8 loops - are special cases of Floer homology for nonlocal conormal boundary conditions. Therefore, we unify the presentation by using this level of generality (see our paper with Portaluri [2]): Given a closed manifold $Q$, we fix a closed submanifold $R$ of $Q \times Q$ and consider the Hamiltonian orbits $x:[0,1] \rightarrow T^{*} Q$ such that $(x(0),-x(1))$ belongs to the conormal bundle $N^{*} R$ of $R$, that is to the set of covectors in $T^{*}(Q \times Q)$ which are based at $R$ and annihilate every vector which is tangent to $R$. The Floer homology on $T^{*} Q$ associated to $N^{*} R$ is denoted by $H F_{*}^{R}\left(T^{*} Q\right)$. The standard Floer homology on $T^{*} M$ with periodic boundary conditions corresponds to the choice $Q=M$ and $R=\Delta_{M}$, the diagonal in $M \times M$. Floer homology for based loops (or Dirichlet boundary conditions) corresponds to choosing $Q=M$ and $R$ to consist of the point $\left(q_{0}, q_{0}\right)$. Finally, in Floer homology for figure-8 loops we choose $Q=M \times M$ and $R=\Delta_{M}^{(4)}$, the set of quadruples $(q, q, q, q)$ in $M^{4}$.

In Section 4, we start by recalling the construction of the isomorphism

$$
\Phi_{*}^{R}: H_{*}\left(P_{R}(Q)\right) \stackrel{\cong}{\longrightarrow} H F_{*}^{R}\left(T^{*} Q\right)
$$

where $\left.P_{R}(Q)\right)$ is the space of continuous paths $\gamma:[0,1] \rightarrow Q$ such that $(\gamma(0), \gamma(1)) \in R$. Then we prove Theorems A and B, as corollaries of chain level results (Theorem 4.4 and Theorem 4.1) involving the Morse complex of the Lagrangian action functional. In Section 4.6, we complete the picture by showing how the other homomorphisms which appear in diagram (0-7) can be described in a Floer theoretical way.

The linear Fredholm theory used in these sections is described in Section 5, whereas Section 6 contains compactness and removal of singularities results, together with the proofs of three cobordism statements from Section 3 and Section 4.

Some of the proofs are based on standard techniques in Floer homology, and in this case we just refer to the literature. However, there are a few key points where we need to introduce some new ideas. We conclude this introduction by briefly describing these ideas. 
Riemann surfaces as quotients of strips with slits The definition of the pair-of-pants product requires extending the Cauchy-Riemann type equation (0-2) to the pair-of-pants surface. The Cauchy-Riemann operator $\partial_{s}+J \partial_{t}$ naturally extends to any Riemann surface, by letting it take value into the vector bundle of antilinear one-forms. The zeroorder term $-J X_{H}(t, u)$ instead does not have a natural extension when the Riemann surface does not have a global coordinate $z=s+i t$. The standard way to overcome this difficulty is to make this zero-order term act only on the cylindrical ends of the pair-of-pants surface - which do have a global coordinate $z=s+i t$ - by multiplying the Hamiltonian by a cut-off function making it vanish far from the cylindrical ends (see the second author's thesis [46] and McDuff and Salamon [38, Section 12.2], but see also Seidel [47] for a different approach). This construction does not cause problems when dealing with compact symplectic manifolds as in the above-mentioned reference, but in the case of the cotangent bundle it would create problems with compactness of the spaces of solutions. In fact, on one hand cutting off the Hamiltonian destroys the identity relating the energy of the solution with the oscillation of the action functional, on the other hand our $C^{0}$-estimate for the solutions requires coercive Hamiltonians.

We overcome this difficulty by a different - and we believe more natural - way of extending the zero-order term. We describe the pair-of-pants surface - as well as the other Riemann surfaces we need to deal with - as the quotient of an infinite strip with a slit - or more slits in the case of more general Riemann surfaces. At the end of the slit we use a chart given by the square root map. In this way, the Riemann surface is still seen as a smooth object, but it carries a global coordinate $z=s+i t$ with singularities. This global coordinate allows to extend the zero-order term without cutting off the Hamiltonian, and preserving the energy identity. See Section 3.2 below.

\section{Cauchy-Riemann operators on strips with jumping boundary conditions When} using the above description for the Riemann surfaces, the problems we are looking at can be described in a unified way as Cauchy-Riemann type equations on a strip, with Lagrangian boundary conditions presenting a finite number of jumps. In Section 5 we develop a complete linear theory for such problems, in the case of Lagrangian boundary conditions of conormal type. These are the kind of conditions which occur naturally on cotangent bundles. Once the proper Sobolev setting has been chosen, the proof of the Fredholm property for such operators is standard. The computation of the index instead is reduced to a Liouville type statement, proved in Section 5.5.

These linear results have the following consequence. Let $R_{0}, \ldots, R_{k}$ be submanifolds of $Q \times Q$, such that $R_{j-1}$ and $R_{j}$ intersect cleanly, for every $j=1, \ldots, k$. Let $-\infty=s_{0}<s_{1}<\cdots<s_{k}<s_{k+1}=+\infty$, and consider the space $M$ consisting of the maps $u: \mathbb{R} \times[0,1] \rightarrow T^{*} Q$ solving the Cauchy-Riemann type equation (0-2), 
satisfying the boundary conditions

$$
(u(s, 0),-u(s, 1)) \in N^{*} R_{j} \quad \forall s \in\left[s_{j}, s_{j+1}\right], \forall j=0, \ldots, k,
$$

and converging to Hamiltonian orbits $x^{-}$and $x^{+}$for $s \rightarrow-\infty$ and $s \rightarrow+\infty$. The results of Section 5 imply that for a generic choice of the Hamiltonian $H$ the space $\mathcal{M}$ is a manifold of dimension

$$
\operatorname{dim} \mu=\mu^{R_{0}}\left(x^{-}\right)-\mu^{R_{k}}\left(x^{+}\right)-\sum_{j=1}^{k}\left(\operatorname{dim} R_{j-1}-\operatorname{dim} R_{j-1} \cap R_{j}\right) .
$$

Here $\mu^{R_{0}}\left(x^{-}\right)$and $\mu^{R_{k}}\left(x^{+}\right)$are the Maslov indices of the Hamiltonian orbits $x^{-}$ and $x^{+}$, with boundary conditions $\left(x^{-}(0),-x^{-}(1)\right) \in N^{*} R_{0},\left(x^{+}(0),-x^{+}(1)\right) \in$ $N^{*} R_{k}$, suitably shifted so that in the case of a fiberwise convex Hamiltonian they coincide with the Morse indices of the corresponding critical points $\gamma^{-}$and $\gamma^{+}$of the Lagrangian action functional on the spaces of paths satisfying $\left(\gamma^{-}(0), \gamma^{-}(1)\right) \in R_{0}$ and $\left(\gamma^{+}(0), \gamma^{+}(1)\right) \in R_{k}$, respectively. Similar formulas hold for problems on the half-strip. See Section 5.10 for precise statements. Different approaches to jumping Lagrangian boundary conditions can be found in Ivashkovich and Shevchishin [34], in Wehrheim and Woodward [56; 58; 57; 55] and in Cieliebak, Ekholm and Latschev [11].

Cobordism arguments The main results of this paper always reduce to the fact that certain diagrams involving homomorphisms defined either in a Floer or in a Morse theoretical way should commute up to a chain homotopy. The proof of such a commutativity is based on cobordism arguments, saying that a given solution of a certain Problem 1 can be "continued" by a unique one-parameter family of solutions of a certain Problem 2, and that this family of solutions converges to a solution of a certain Problem 3. In many situations such a statement can be proved by the classical gluing argument in Floer theory: One finds the one-parameter family of solutions of Problem 2 by using the given solution of Problem 1 to construct an approximate solution, to be used as the starting point of a Newton iteration scheme which converges to a true solution. When this is the case, we just refer to the literature. However, we encounter three situations in which the standard arguments do not apply, one reason being that we face a Problem 2 involving a Riemann surface whose conformal structure is varying with the parameter: this occurs when proving that the pair-of-pants product factorizes through the figure-8 Floer homology (Section 3.4), that the Pontrjagin product corresponds to the triangle product (Section 4.2), and that the homomorphism $e_{!} \circ \times$ corresponds to its Floer homological counterpart (Section 4.4). We manage to reduce the former two statements to the standard implicit function theorem (see Section 6.3 and Section 6.4). The proof of the latter statement is more involved, because in 
this case the solution of Problem 2 we are looking for cannot be expected to be even $C^{0}$-close to the solution of Problem 1 we start with. We overcome this difficulty by the following algebraic observation: In order to prove that two chain maps $\varphi, \psi: C \rightarrow C^{\prime}$ are chain homotopic, it suffices to find a chain homotopy between the chain maps $\varphi \otimes \psi$ and $\psi \otimes \varphi$, and to find an element $\epsilon \in C_{0}$ and a chain map $\delta$ from the complex $C^{\prime}$ to the trivial complex $(\mathbb{Z}, 0)$ such that $\delta(\varphi(\epsilon))=\delta(\psi(\epsilon))=1$ (see Lemma 4.6 below). In our situation, the chain homotopy between $\varphi \otimes \psi$ and $\psi \otimes \varphi$ is easier to find, by using a localization argument and the implicit function theorem (see Section 6.5). This argument is somehow reminiscent of an alternative way suggested by H Hofer to prove standard gluing results in Floer homology. The construction of the element $\epsilon$ and of the chain map $\delta$ is presented in Section 4.4, together with the proof of the required algebraic identity. This is done by considering special Hamiltonian systems having a hyperbolic equilibrium point.

The main results of this paper were announced in our paper [3]. Related results concerning the equivariant loop product and its interpretation in the symplectic field theory of unit cotangent bundles have been announced by Cieliebak and Latschev [12].

Acknowledgements We wish to thank the Max Planck Institute for Mathematics in the Sciences of Leipzig and the Department of Mathematics at Stanford University, and in particular Yasha Eliashberg, for their kind hospitality. We are also indebted to Ralph Cohen and Helmut Hofer for many fruitful discussions. The first author thanks the Humboldt Foundation for financial support in the form of a Humboldt Research Fellowship for Experienced Researchers. The second author thanks the Deutsche Forschungsgemeinschaft for the support by the grant DFG SCHW 892/2-3.

\section{The Pontrjagin and the loop products}

\subsection{The Pontrjagin product}

Given a topological space $M$ and a point $q_{0} \in M$, we denote by $\Omega\left(M, q_{0}\right)$ the space of loops on $M$ based at $q_{0}$, that is

$$
\Omega\left(M, q_{0}\right):=\left\{\gamma \in C^{0}(\mathbb{T}, M) \mid \gamma(0)=q_{0}\right\},
$$

endowed with the compact-open topology. Here $\mathbb{T}=\mathbb{R} / \mathbb{Z}$ is the circle parameterized by the interval $[0,1]$. The concatenation

$$
\Gamma\left(\gamma_{1}, \gamma_{2}\right)(t):= \begin{cases}\gamma_{1}(2 t) & \text { for } 0 \leq t \leq 1 / 2, \\ \gamma_{2}(2 t-1) & \text { for } 1 / 2 \leq t \leq 1,\end{cases}
$$


maps $\Omega\left(M, q_{0}\right) \times \Omega\left(M, q_{0}\right)$ continuously into $\Omega\left(M, q_{0}\right)$. The constant loop $q_{0}$ is a homotopy unit for $\Gamma$, meaning that the maps $\gamma \mapsto \Gamma\left(q_{0}, \gamma\right)$ and $\gamma \mapsto \Gamma\left(\gamma, q_{0}\right)$ are homotopic to the identity map. Moreover, $\Gamma$ is homotopy associative, meaning that $\Gamma \circ(\Gamma \times \mathrm{id})$ and $\Gamma \circ(\mathrm{id} \times \Gamma)$ are homotopic. Therefore, $\Gamma$ defines the structure of an $H$-space on $\Omega\left(M, q_{0}\right)$.

We denote by $H_{*}$ the singular homology functor with integer coefficients. The composition

$$
\begin{aligned}
H_{j}\left(\Omega\left(M, q_{0}\right)\right) \otimes H_{k}\left(\Omega\left(M, q_{0}\right)\right) \stackrel{\times}{\longrightarrow} H_{j+k}\left(\Omega\left(M, q_{0}\right) \times \Omega\left(M, q_{0}\right)\right) & \\
& \stackrel{\Gamma_{*}}{\longrightarrow} H_{j+k}\left(\Omega\left(M, q_{0}\right)\right)
\end{aligned}
$$

where the first arrow is the exterior homology product, is by definition the Pontrjagin product

$$
\text { \#: } H_{j}\left(\Omega\left(M, q_{0}\right)\right) \otimes H_{k}\left(\Omega\left(M, q_{0}\right)\right) \rightarrow H_{j+k}\left(\Omega\left(M, q_{0}\right)\right) .
$$

The fact that $q_{0}$ is a homotopy unit for $\Gamma$ implies that $\left[q_{0}\right] \in H_{0}\left(\Omega\left(M, q_{0}\right)\right)$ is the identity element for the Pontrjagin product. The fact that $\Gamma$ is homotopy associative implies that the Pontrjagin product is associative. Therefore, the product \# makes the singular homology of $\Omega\left(M, q_{0}\right)$ a graded ring. In general, it is a noncommutative graded ring. See for instance tom Dieck, Kamps and Puppe [19] for more information on $H$-spaces and the Pontrjagin product.

\subsection{The Chas-Sullivan loop product}

We denote by $\Lambda(M):=C^{0}(\mathbb{T}, M)$ the space of free loops on $M$. Under the assumption that $M$ is an oriented $n$-dimensional manifold, it is possible to use the concatenation map $\Gamma$ to define a product of degree $-n$ on $H_{*}(\Lambda(M))$. In order to describe the construction, we need to recall the definition of the Umkehr map.

Let $\mathcal{M}$ be a (possibly infinite-dimensional) smooth Banach manifold, and let $e: \mathcal{M}_{0} \hookrightarrow$ $\mathcal{M}$ be a smooth closed embedding, which we assume to be $n$-codimensional and cooriented. In other words, $\mathcal{M}_{0}$ is a closed submanifold of $\mathcal{M}$ whose normal bundle $N \mathcal{M}_{0}:=\left.T \mathcal{M}\right|_{\mathcal{M}_{0}} / T \mathcal{M}_{0}$ has dimension $n$ and is oriented. The tubular neighborhood theorem provides us with a homeomorphism $u: \mathcal{U} \rightarrow N \mathcal{M}_{0}$, uniquely determined up to isotopy, of an open neighborhood of $\mathcal{M}_{0}$ onto $N \mathcal{M}_{0}$, mapping $\mathcal{M}_{0}$ identically onto the zero section of $N \mathcal{M}_{0}$, that we also denote by $\mathcal{M}_{0}$ (see Lang [35, IV, Sections 5-6] if $\mathcal{M}$ admits smooth partitions of unity - for instance, if it is a Hilbert manifold - then $u$ can be chosen to be a smooth diffeomorphism). The Umkehr map e! associated to 
the embedding $e$ is defined to be the composition

$$
\begin{array}{r}
H_{j}(\mathcal{M}) \longrightarrow H_{j}\left(\mathcal{M}, \mathcal{M} \backslash \mathcal{M}_{0}\right) \stackrel{\cong}{\longrightarrow} H_{j}\left(\mathcal{U}, \mathcal{U} \backslash \mathcal{M}_{0}\right) \stackrel{u_{*}}{\longrightarrow} H_{j}\left(N \mathcal{M}_{0}, N \mathcal{M}_{0} \backslash \mathcal{M}_{0}\right) \\
\stackrel{\tau}{\longrightarrow} H_{j-n}\left(\mathcal{M}_{0}\right),
\end{array}
$$

where the first arrow is induced by the inclusion, the second one is the isomorphism given by excision, and the last one is the Thom isomorphism associated to the $n-$ dimensional oriented vector bundle $N \mathcal{M}_{0}$, that is, the cap product with the Thom class $\tau_{N \mathcal{M}_{0}} \in H^{n}\left(N \mathcal{M}_{0}, N \mathcal{M}_{0} \backslash \mathcal{M}_{0}\right)$.

We recall that if $M$ is an $n$-dimensional manifold, $\Lambda(M)$ is an infinite dimensional smooth manifold modeled on the Banach space $C^{0}\left(\mathbb{T}, \mathbb{R}^{n}\right)$. The set $\Theta(M)$ of pairs of loops with the same initial point (figure-8 loops),

$$
\Theta(M):=\left\{\left(\gamma_{1}, \gamma_{2}\right) \in \Lambda(M) \times \Lambda(M) \mid \gamma_{1}(0)=\gamma_{2}(0)\right\},
$$

is the inverse image of the diagonal $\Delta_{M}$ of $M \times M$ by the smooth submersion

$$
\mathrm{ev} \times \mathrm{ev}: \Lambda(M) \times \Lambda(M) \rightarrow M \times M, \quad\left(\gamma_{1}, \gamma_{2}\right) \mapsto\left(\gamma_{1}(0), \gamma_{2}(0)\right) .
$$

Therefore, $\Theta(M)$ is a closed smooth submanifold of $\Lambda(M) \times \Lambda(M)$, and its normal bundle $N \Theta(M)$ is $n$-dimensional, being isomorphic to the pullback of the normal bundle $N \Delta_{M}$ of $\Delta_{M}$ in $M \times M$ by the map ev $\times$ ev. The Banach manifold $\Lambda(M)$ does not admit smooth partitions of unity (actually, the Banach space $C^{0}\left(\mathbb{T}, \mathbb{R}^{n}\right)$ does not admit nonzero functions of class $C^{1}$ with bounded support). So in general a closed submanifold of $\Lambda(M)$, or of $\Lambda(M) \times \Lambda(M)$, will not have a smooth tubular neighborhood. However, it would not be difficult to show that the submanifold $\Theta(M)$ and all the submanifolds we consider in this paper do have a smooth tubular neighborhood, which can be constructed explicitly by using the exponential map and the tubular neighborhood theorem on finite-dimensional manifolds. If moreover $M$ is oriented, so is $N \Delta_{M}$ and thus also $N \Theta(M)$. Notice also that the concatenation map $\Gamma$ is well-defined and smooth from $\Theta(M)$ into $\Lambda(M)$. If we denote by $e$ the inclusion of $\Theta(M)$ into $\Lambda(M) \times \Lambda(M)$, the Chas-Sullivan loop product [9] is defined by the composition

$$
\begin{aligned}
H_{j}(\Lambda(M)) \otimes H_{k}(\Lambda(M)) \stackrel{\times}{\longrightarrow} H_{j+k}(\Lambda(M) \times \Lambda(M)) \stackrel{e_{!}}{\longrightarrow} H_{j+k-n}(\Theta(M)) & \stackrel{\Gamma_{*}}{\longrightarrow} H_{j+k-n}(\Lambda(M)),
\end{aligned}
$$

and it is denoted by

$$
\text { ○: } H_{j}(\Lambda(M)) \otimes H_{k}(\Lambda(M)) \rightarrow H_{j+k-n}(\Lambda(M)) .
$$


We denote by c: $M \rightarrow \Lambda(M)$ the map which associates to every $q \in M$ the constant loop $q$ in $\Lambda(M)$. A simple homotopy argument shows that the image of the fundamental class $[M] \in H_{n}(M)$ under the homomorphism $\mathrm{c}_{*}$ is a unit for the loop product: $\alpha \circ \mathrm{c}_{*}[M]=\mathrm{c}_{*}[M] \circ \alpha=\alpha$ for every $\alpha \in H_{*}(\Lambda(M))$. Since $\Gamma \circ(\mathrm{id} \times \Gamma)$ and $\Gamma \circ(\Gamma \times$ id $)$ are homotopic on the space of triplets of loops with the same initial points, the loop product turns out to be associative. Finally, notice that the maps $\left(\gamma_{1}, \gamma_{2}\right) \mapsto \Gamma\left(\gamma_{1}, \gamma_{2}\right)$ and $\left(\gamma_{1}, \gamma_{2}\right) \mapsto \Gamma\left(\gamma_{2}, \gamma_{1}\right)$ are homotopic on $\Theta(M)$, by the homotopy

$$
\Gamma_{s}\left(\gamma_{1}, \gamma_{2}\right)(t):= \begin{cases}\gamma_{2}(2 t-s) & \text { if } 0 \leq t \leq s / 2, \text { or }(s+1) / 2 \leq t \leq 1, \\ \gamma_{1}(2 t-s) & \text { if } s / 2 \leq t \leq(s+1) / 2\end{cases}
$$

This fact implies the following commutation rule

$$
\beta \circ \alpha=(-1)^{(|\alpha|-n)(|\beta|-n)} \alpha \circ \beta,
$$

for every $\alpha, \beta \in H_{*}(\Lambda(M))$.

In order to get a product of degree zero, it is convenient to shift the grading by $n$, obtaining the graded group

$$
\mathbb{H}_{j}(\Lambda(M)):=H_{j+n}(\Lambda(M)),
$$

which becomes a graded commutative ring with respect to the loop product (commutativity has to be understood in the graded sense, that is $\left.\beta \circ \alpha=(-1)^{|\alpha||\beta|} \alpha \circ \beta\right)$.

\subsection{Relationship between the two products}

If $M$ is an oriented $n$-dimensional manifold, we denote by

$$
\bullet: H_{j}(M) \otimes H_{k}(M) \rightarrow H_{j+k-n}(M),
$$

the intersection product on the singular homology of $M$ (which is obtained by composing the exterior homology product with the Umkehr map associated to the embedding of the diagonal into $M \times M)$ ). Shifting again the grading by $n$, we see that the product makes

$$
\mathbb{H}_{j}(M):=H_{j+n}(M)
$$

a commutative graded ring.

Being the inverse image of $q_{0}$ by the submersion ev: $\Lambda(M) \rightarrow M, \operatorname{ev}(\gamma)=\gamma(0)$, $\Omega\left(M, q_{0}\right)$ is a closed submanifold of $\Lambda(M)$, and its normal bundle is $n$-dimensional 
and oriented. If $\mathrm{i}: \Omega\left(M, q_{0}\right) \hookrightarrow \Lambda(M)$ is the inclusion map, we find that the following diagram commutes:

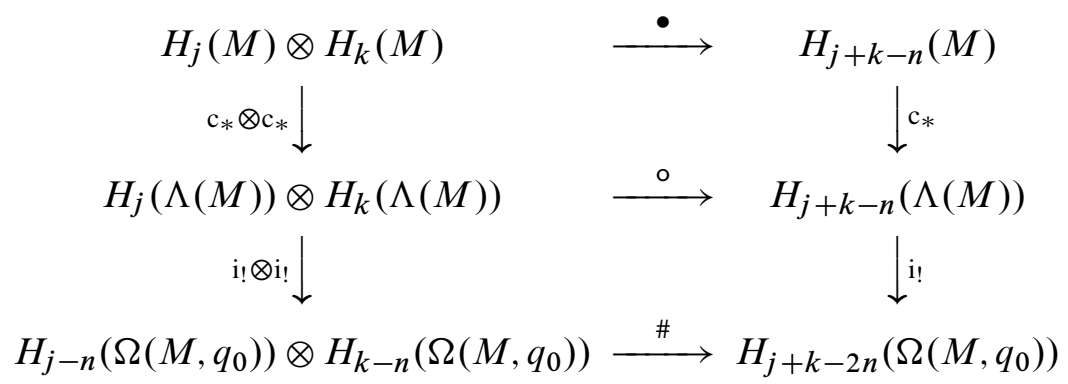

In other words, the maps

$$
\left\{\mathbb{H}_{*}(M), \bullet\right\} \stackrel{c_{*}}{\longrightarrow}\left\{\mathbb{H}_{*}(\Lambda(M)), \circ\right\} \stackrel{\text { i! }}{\longrightarrow}\left\{H_{*}\left(\Omega\left(M, q_{0}\right), \#\right\}\right.
$$

are graded ring homomorphisms. Notice that the homomorphism $\mathrm{c}_{*}$ is always injective onto a direct summand, the map ev being a left inverse of $\mathrm{c}$. Using the spectral sequence associated to the Serre fibration

$$
\Omega(M) \hookrightarrow \Lambda(M) \rightarrow M,
$$

it is possible to compute the ring $\left\{\mathbb{H}_{*}(\Lambda(M)), \circ\right\}$ from the intersection product on $M$ and the Pontrjagin product on $\Omega(M)$, when $M$ is a sphere or a projective space; see Cohen, Jones and Yan [16].

The aim of this paper is to show how the homomorphisms appearing in the diagram above can be described symplectically, in terms of different Floer homologies of the cotangent bundle of $M$, when the manifold $M$ is closed. The first step is to obtain a Morse theoretical chain level description of diagram (1-1), by using suitable Morse functions on some infinite dimensional Hilbert manifolds having the homotopy type of $\Omega\left(M, q_{0}\right), \Lambda(M)$ and $\Theta(M)$.

Remark 1.1 The loop product was defined by Chas and Sullivan in [9], by using intersection theory for transversal chains. The definition we use here is due to Cohen and Jones [15]. See also Chas and Sullivan [10], Sullivan [49], Baas, Cohen and Ramírez [6], Cohen [13], Cohen, Hess and Voronov [14], Ramírez [41], Sullivan [50], Cohen, Klein and Sullivan [17] and Cohen and Schwarz [18] for more information and for other interpretations of this product. 


\section{Morse chain level descriptions}

The aim of this section is to describe the Pontrjagin product \#, the loop product o, and the other homomorphisms appearing in diagram (1-1) in a Morse theoretical way. As it is well known, the singular homology of a (possibly infinite dimensional) manifold $\mathcal{M}$ is isomorphic to the homology of the Morse chain complex associated to a suitable Morse function on $\mathcal{M}$, and most homomorphisms between singular homology groups can be read at the chain level using these Morse complexes. The constructions of the Morse complex and of various homomorphisms between them, in the infinite dimensional setting needed in this paper, are described in the Appendix.

Because of technical reasons, Hilbert manifolds are easier to deal with than Banach manifolds. Therefore, the first thing to do is to replace the Banach manifolds $\Lambda(M)$ (continuous free loops on $M$ ), $\Omega\left(M, q_{0}\right)$ (continuous loops based at $q_{0}$ ), and $\Theta(M)$ (continuous figure-8 loops) by the Hilbert manifolds

$$
\begin{gathered}
\Lambda^{1}(M):=W^{1,2}(\mathbb{T}, M), \quad \Omega^{1}\left(M, q_{0}\right):=\left\{\gamma \in \Lambda^{1}(M) \mid \gamma(0)=q_{0}\right\}, \\
\Theta^{1}(M):=\left\{\left(\gamma_{1}, \gamma_{2}\right) \in \Lambda^{1}(M) \times \Lambda^{1}(M) \mid \gamma_{1}(0)=\gamma_{2}(0)\right\},
\end{gathered}
$$

where $W^{1,2}$ denotes the class of absolutely continuous curves whose derivative is square integrable. The inclusions

$$
\Lambda^{1}(M) \hookrightarrow \Lambda(M), \quad \Omega^{1}\left(M, q_{0}\right) \hookrightarrow \Omega\left(M, q_{0}\right), \quad \Theta^{1}(M) \hookrightarrow \Theta(M),
$$

are homotopy equivalences. Therefore, we can replace $\Lambda(M), \Omega\left(M, q_{0}\right)$, and $\Theta(M)$ by $\Lambda^{1}(M), \Omega^{1}\left(M, q_{0}\right)$, and $\Theta^{1}(M)$ in the constructions of Section 1 (notice that the concatenation of two curves of class $W^{1,2}$ is still of class $\left.W^{1,2}\right)$.

\subsection{The Morse complex of the Lagrangian action functional}

Applying the results of the Appendix, one could find Morse chain level descriptions of all the homomorphisms of diagram (1-1) by using quite a general class of abstract Morse functions on $M, \Lambda^{1}(M), \Omega^{1}\left(M, q_{0}\right)$, and $\Theta^{1}(M)$. However, in order to find a link with Floer theory, we wish to consider a special class of functions on the three latter manifolds, namely the action functionals associated to a (possibly time-dependent) Lagrangian $L$ on $T M$.

Since we wish to consider different boundary conditions, it is useful to unify the presentation by working with general nonlocal conormal boundary conditions. Let $Q$ be a closed manifold (in our applications, $Q$ is either $M$ or $M \times M$ ), and let $R$ be a closed submanifold of $Q \times Q$. Let $L:[0,1] \times T Q \rightarrow \mathbb{R}$ be a smooth function such that there exist real numbers $\ell_{1}>0$ and $\ell_{2}>0$ such that 
(L1) $\nabla_{v v} L(t, q, v) \geq \ell_{1} I$,

(L2) $\left|\nabla_{q q} L(t, q, v)\right| \leq \ell_{2}\left(1+|v|^{2}\right),\left|\nabla_{q v} L(t, q, v)\right| \leq \ell_{2}(1+|v|),\left|\nabla_{v v} L(t, q, v)\right| \leq \ell_{2}$,

for every $(t, q, v)$ in $[0,1] \times T Q$. Here we have fixed a Riemannian metric on $Q$, with associated norm $|\cdot|$, and $\nabla_{v}, \nabla_{q}$ denote the vertical and horizontal components of the gradient with respect to this metric and to the induced metric on $T Q$. The fact that $Q$ is compact implies that these conditions do not depend on the choice of the metric. Conditions (L1) implies that $L$ is strictly fiberwise convex and grows at least quadratically in $v$. Condition (L2) implies that $L$ grows at most quadratically in $v$.

The Euler-Lagrange equation for a curve $\gamma:[0,1] \mapsto Q$ can be written in local coordinates as

$$
\frac{d}{d t} \partial_{v} L\left(t, \gamma(t), \gamma^{\prime}(t)\right)=\partial_{q} L(t, \gamma(t), \gamma(t))
$$

and by (L1) the corresponding second order Cauchy problem is locally well-posed. By (L2), the Lagrangian action functional

$$
\mathbb{S}_{L}(\gamma):=\int_{0}^{1} L\left(t, \gamma(t), \gamma^{\prime}(t)\right) d t
$$

is continuously differentiable on the Hilbert manifold $W^{1,2}([0,1], Q)$. Moreover, it is twice Gateaux differentiable at every curve $\gamma$, but it is everywhere twice Fréchét differentiable if and only if for every $(t, q)$ the function $v \mapsto L(t, q, v)$ is a polynomial of degree at most two on $T_{q} Q$ [5]. In the latter case, if we also assume (L1), the homogeneous part of degree two in $v \mapsto L(t, q, v)$ should be a positive quadratic form, so $L$ is an electromagnetic Lagrangian, ie it has the form

$$
L(t, q, v)=\frac{1}{2}\langle A(t, q) v, v\rangle+\langle\alpha(t, q), v\rangle-V(t, q),
$$

where $\langle\cdot, \cdot\rangle$ denotes the duality pairing, $A(t, q): T_{q} Q \rightarrow T_{q}^{*} Q$ is a positive symmetric linear mapping smoothly depending on $(t, q)$ (the kinetic energy), $\alpha$ is a smoothly time dependent one-form (the magnetic potential), and $V$ is a smooth function (the scalar potential). In this case, the action functional $\mathbb{S}_{L}$ is actually smooth. In some situations, one can restrict the attention to electromagnetic Lagrangians, as in [2]. However, in view of the transversality issues coming from Floer homology, it is convenient to work with the more general class of Lagrangians which satisfy (L1) and (L2). The reason is that some issues concerning $J$-holomorphic curves on cotangent bundles become much simpler when $J$ is the Levi-Civita almost complex structure (see Section 3, and in particular Remark 3.3). Therefore, in this paper we prefer not to perturb the Levi-Civita $J$, and to achieve the transversality needed in Floer theory by perturbing the Hamiltonian, or equivalently, the Lagrangian. The class of electromagnetic Lagrangians 
is too rigid for this purpose, whereas the fact that conditions (L1) and (L2) are stable with respect to $C^{2}$-small perturbations of $L$ allows to achieve transversality within this class.

The closed submanifold $R$ of $Q \times Q$ defines the following boundary conditions for solutions $\gamma:[0,1] \times Q$ of the Euler-Lagrange equation (2-1):

$$
\begin{gathered}
(\gamma(0), \gamma(1)) \in Q, \\
D_{v} L\left(0, \gamma(0), \gamma^{\prime}(0)\right)\left[\xi_{0}\right]=D_{v} L\left(1, \gamma(1), \gamma^{\prime}(1)\right)\left[\xi_{1}\right], \forall\left(\xi_{0}, \xi_{1}\right) \in T_{(\gamma(0), \gamma(1))} R .
\end{gathered}
$$

These conditions take a simpler form when read in the Hamiltonian formulation; see Section 3.1. The set of solutions of (2-1)-(2-3) is denoted by $\mathscr{P}^{R}(L)$. The elements of $\mathscr{P}^{R}(L)$ are precisely the critical points of $\mathbb{S}_{L}^{R}$, that is the restriction of the Lagrangian action functional $\mathbb{S}_{L}$ to the Hilbert submanifold

$$
W_{R}^{1,2}([0,1], Q):=\left\{\gamma \in W^{1,2}([0,1], Q) \mid(\gamma(0), \gamma(1)) \in R\right\} .
$$

A critical point $\gamma$ of $\mathbb{S}_{L}^{R}$ has always finite Morse index

$$
i\left(\gamma ; \mathbb{S}_{L}^{R}\right)<+\infty
$$

and it is nondegenerate if and only if there are no nonzero Jacobi vector fields along $\gamma$ (ie solutions of the linear system which is obtained by linearizing (2-1) along $\gamma$ ) which satisfy the linear boundary conditions that one finds by linearizing (2-2)-(2-3).

Let us assume that all the elements of $\mathscr{P}^{R}(L)$ are nondegenerate. This assumption holds for a generic choice of $L$, in several reasonable topologies (a subset of a topological space is said to be generic if it is a countable intersection of open and dense sets). Although the functional $\mathbb{S}_{L}^{R}$ is in general not twice Fréchét differentiable, it admits a smooth pseudo-gradient $X$, in the sense of Appendix A.1, which satisfies the conditions (X1)-(X4). This fact is proved in [5]. Hence, such a functional has a well-defined Morse complex $M_{*}\left(\mathbb{S}_{L}^{R}\right)$, whose homology $H M_{*}\left(\mathbb{S}_{L}^{R}\right)$ is isomorphic to the singular homology of $W_{R}^{1,2}([0,1], Q)$, as explained in Appendix A.1.

In this paper, we are interested in the following three boundary conditions.

Periodic boundary conditions Here $Q=M$ and $R=\Delta_{M}$ is the diagonal in $M \times M$. Under the extra assumption that $L \in C^{\infty}([0,1] \times T M)$ extends as a smooth 1 -periodic function on $\mathbb{R} \times T M$, the set $\mathscr{P}^{\Delta_{M}}(L)$ is precisely the set 1 -periodic solutions of the Euler-Lagrange equation (2-1). We denote such a set also by $\mathscr{P}^{\Lambda}(L)$, and the restricted functional $\mathbb{S}_{L}^{\Delta_{M}}$ by $\mathbb{S}_{L}^{\Lambda}: \Lambda^{1}(M) \rightarrow \mathbb{R}$, noticing that $W_{\Delta_{M}}^{1,2}([0,1], M)=\Lambda^{1}(M)$. The nondegeneracy condition is just: 
$(\mathrm{L} 0)^{\Lambda}$ Every solution $\gamma \in \mathscr{P}^{\Lambda}(L)$ is nondegenerate, meaning that there are no nonzero periodic Jacobi vector fields along $\gamma$.

The Morse index of a critical point $\gamma$ of $\mathbb{S}_{L}^{\Lambda}$ is denoted by $i^{\Lambda}(\gamma)$, or by $i^{\Lambda}(\gamma ; L)$ when the choice of the Lagrangian $L$ is not clear from the context. The homology of the Morse complex $M_{*}\left(\mathbb{S}_{L}^{\Lambda}\right)$ is isomorphic to the singular homology of $\Lambda^{1}(M)$, hence to the singular homology of $\Lambda(M)$.

Dirichlet boundary conditions Here $Q=M$ and $R$ consists of the single point $\left(q_{0}, q_{0}\right)$, for some fixed $q_{0} \in M$. The set $\mathscr{P}^{\left(q_{0}, q_{0}\right)}(L)$, also denoted $\mathscr{P}^{\Omega}(L)$, is precisely the set of solutions $\gamma:[0,1] \rightarrow M$ of the Euler-Lagrange equation (2-1) with $\gamma(0)=\gamma(1)=q_{0}$. The space of curves $W_{\left(q_{0}, q_{0}\right)}^{1,2}([0,1], M)$ is just $\Omega^{1}\left(M, q_{0}\right)$, and $\mathbb{S}_{L}^{\Omega}: \Omega^{1}\left(M, q_{0}\right) \rightarrow \mathbb{R}$ also denotes the functional $\mathbb{S}_{L}^{\left(q_{0}, q_{0}\right)}$. The nondegeneracy condition is now:

$(\mathrm{L} 0)^{\Omega}$ Every solution $\gamma \in \mathscr{P}^{\Omega}(L)$ is nondegenerate, meaning that there are no nonzero Jacobi vector fields along $\gamma$ which vanish for $t=0$ and for $t=1$.

The nondegeneracy condition $(\mathrm{L} 0)^{\Omega}$ is generic also in the smaller class of autonomous Lagrangians $L$, whereas (L0) ${ }^{\Lambda}$ requires $L$ to be explicitly time-dependent, because if $L$ is autonomous then $\gamma^{\prime}$ is always a Jacobi vector field along $\gamma$. The Morse index of a critical point $\gamma$ of $\mathbb{S}_{L}^{\Omega}$ is denoted by $i^{\Omega}(\gamma)=i^{\Omega}(\gamma ; L)$. The homology of the Morse complex $M_{*}\left(\mathbb{S}_{L}^{\Omega}\right)$ is isomorphic to the singular homology of $\Omega^{1}\left(M, q_{0}\right)$, hence to the singular homology of $\Omega\left(M, q_{0}\right)$.

Figure- 8 boundary conditions Here $Q=M \times M$ and $R=\Delta_{M}^{(4)}$ is the fourth diagonal in $M^{4}$ :

$$
\Delta_{M}^{(4)}:=\{(q, q, q, q) \mid q \in M\} .
$$

In this case, it is convenient (although not necessary) to chose $L$ of the form $L=$ $L_{1} \oplus L_{2}$, where

$L_{1} \oplus L_{2}(t, q, v):=L_{1}\left(t, q_{1}, v_{1}\right)+L_{2}\left(t, q_{2}, v_{2}\right)$,

$$
\forall t \in[0,1], \forall q=\left(q_{1}, q_{2}\right) \in M \times M, \forall v=\left(v_{1}, v_{2}\right) \in T_{\left(q_{1}, q_{2}\right)} M \times M,
$$

so that the elements of $\mathscr{P}_{M}^{(4)}(L)$ are the pairs of $M$-valued curves $\left(\gamma_{1}, \gamma_{2}\right)$ such that each $\gamma_{j}$ solves the Euler-Lagrange equation induced by $L_{j}$, and such that the boundary conditions

$$
\gamma_{1}(0)=\gamma_{1}(1)=\gamma_{2}(0)=\gamma_{2}(1), \quad \sum_{i=0}^{1} \sum_{j=1}^{2}(-1)^{i} D_{v} L_{j}\left(i, \gamma_{j}(i), \gamma_{j}^{\prime}(i)\right)=0
$$


hold. The set of such solutions is denoted also by $\mathscr{P}^{\Theta}\left(L_{1} \oplus L_{2}\right)$. The space of curves

$$
W_{\Delta_{M}^{(4)}}^{1,2}([0,1], M \times M)
$$

coincides with $\Theta^{1}(M)$, and we simply denote the functional

$$
\mathbb{S}_{L}^{\Delta_{M}^{(4)}} \quad \text { by } \quad \mathbb{S}_{L}^{\Theta}: \Theta^{1}(M) \rightarrow \mathbb{R} .
$$

The corresponding nondegeneracy condition is:

(L0) ${ }^{\Theta}$ Every solution $\left(\gamma_{1}, \gamma_{2}\right) \in \mathscr{P}^{\Theta}\left(L_{1} \oplus L_{2}\right)$ is nondegenerate, meaning that there are no nonzero pairs of Jacobi vector fields $\left(\xi_{1}, \xi_{2}\right)$ along $\left(\gamma_{1}, \gamma_{2}\right)$ such that

$$
\begin{gathered}
\xi_{1}(0)=\xi_{1}(1)=\xi_{2}(0)=\xi_{2}(1), \\
\sum_{i=0}^{1} \sum_{j=1}^{2}\left(D_{q v} L_{j}\left(i, \gamma_{j}(i), \gamma_{j}^{\prime}(i)\right) \xi_{j}(i)+D_{v v} L_{j}\left(i, \gamma_{j}(i), \gamma_{j}^{\prime}(i)\right) \xi_{j}^{\prime}(i)\right)=0 .
\end{gathered}
$$

This condition allows both $L_{1}$ and $L_{2}$ to be autonomous. It also allows $L_{1}=L_{2}$, but this excludes the autonomous case (otherwise pairs $(\gamma, \gamma)$ with $\gamma \in \mathscr{P} \Lambda\left(L_{1}\right)=$ $\mathscr{P}^{\Lambda}\left(L_{2}\right)$ nonconstant would violate $\left.(\mathrm{L} 0)^{\Theta}\right)$. The Morse index of a critical point $\gamma$ of $\mathbb{S}_{L_{1} \oplus L_{2}}^{\Theta}$ is denoted by $i^{\Theta}(\gamma)=i^{\Theta}\left(\gamma ; L_{1} \oplus L_{2}\right)$. The homology of the Morse complex $M_{*}\left(\mathbb{S}_{L_{1} \oplus L_{2}}^{\Theta}\right)$ is isomorphic to the singular homology of $\Theta^{1}(M)$, hence to the singular homology of $\Theta(M)$.

\subsection{Morse description of the homomorphisms c, cv and i!}

The aim of this section is to describe the chain maps between Morse complexes that are induced by the smooth maps

$$
\begin{gathered}
\mathrm{c}: M \rightarrow \Lambda^{1}(M), c(q)(t) \equiv q, \quad \text { ev: } \Lambda^{1}(M) \rightarrow M, \operatorname{ev}(\gamma)=\gamma(0), \\
\text { i: } \Omega^{1}\left(M, q_{0}\right) \hookrightarrow \Lambda^{1}(M) .
\end{gathered}
$$

Everything follows from the abstract results of Appendix A.2 and A.4, provided that we check the transversality conditions which are required there.

It is straightforward to apply the results of Appendix A.2 to describe the homomorphisms

$$
\mathrm{c}_{*}: H_{k}(M) \rightarrow H_{k}\left(\Lambda^{1}(M)\right) \text { and } \quad \mathrm{ev}_{*}: H_{k}\left(\Lambda^{1}(M)\right) \rightarrow H_{k}(M),
$$

in terms of the Morse complexes of $\mathbb{S}_{L}^{\Lambda}$ and of a smooth Morse function $f$ on $M$. Indeed, in the case of $\mathrm{c}_{*}$ one imposes the condition

$$
q \in \operatorname{crit}(f) \quad \Longrightarrow \quad \mathrm{c}(q) \notin \mathscr{P}^{\Lambda}(L),
$$

which guarantees (A-2) and (A-3) (given any Lagrangian $L$ satisfying (L0) ${ }^{\Lambda}$, one can always find a Morse function $f$ on $M$ such that (2-5) holds, simply because (L0) ${ }^{\Lambda}$ 
implies that the Euler-Lagrange equation has finitely many constant solutions). Then one can find a metric $g$ on $M$ and a smooth pseudo-gradient $X_{L}^{\Lambda}$ for $\mathbb{S}_{L}^{\Lambda}$ such that the vector fields $X_{L}^{\Lambda}$ and $-\operatorname{grad}_{g} f$ satisfy the Morse-Smale condition, and the restriction of $c$ to the unstable manifold of each $q \in \operatorname{crit}(f)$ is transverse to the stable manifold of each $\gamma \in \operatorname{crit}\left(\mathbb{S}_{L}^{\Lambda}\right)$. When $i(q ; f)=i^{\Lambda}(\gamma)$, the intersection

$$
W^{u}\left(q ;-\operatorname{grad}_{g} f\right) \cap \mathrm{c}^{-1}\left(W^{s}\left(\gamma ; X_{L}^{\Lambda}\right)\right)
$$

consists of finitely many points, each of which comes with an orientation sign \pm 1 . The algebraic sums $n_{\mathrm{c}}(q, \gamma)$ of these signs provide us with the coefficients of a chain map

$$
M_{k} \mathrm{c}: M_{k}(f) \rightarrow M_{k}\left(\mathbb{S}_{L}^{\Lambda}\right),
$$

which in homology induces the homomorphism $\mathrm{c}_{*}$.

The map ev: $\Lambda^{1}(M) \rightarrow M$ is a submersion, so (A-2) holds automatically. Condition (A-3) instead is implied by

$$
\gamma \in \mathscr{P}^{\Lambda}(L) \quad \Longrightarrow \quad \gamma(0) \notin \operatorname{crit}(f),
$$

which again holds for a generic $f$, given $L$. Then, for a generic metric $g$ on $M$ and a generic pseudo-gradient $X_{L}^{\Lambda}$ for $\mathbb{S}_{L}^{\Lambda}$, the intersections

$$
W^{u}\left(\gamma ; X_{L}^{\Lambda}\right) \cap \mathrm{ev}^{-1}\left(W^{s}\left(q ;-\operatorname{grad}_{g} f\right)\right),
$$

consist of finitely many oriented points, which add up to the integer $n_{\mathrm{ev}}(\gamma, q)$, for every $\gamma \in \mathscr{P}^{\Lambda}(L)$ and $q \in \operatorname{crit}(f)$, such that $i^{\Lambda}(\gamma)=i(q ; f)$. These integers are the coefficients of a chain map

$$
M_{k} \mathrm{ev}: M_{k}\left(\mathbb{S}_{L}^{\Lambda}\right) \rightarrow M_{k}(f),
$$

which in homology induces the homomorphism $\mathrm{ev}_{*}$.

Remark 2.1 The fact that $\mathrm{ev}_{*} \circ \mathrm{c}_{*}=\mathrm{id}_{H_{*}(M)}$, together with the fact that the Morse complex is free, implies that $M_{*} \mathrm{ev} \circ M_{*} \mathrm{c}$ is chain homotopic to the identity on $M_{*}(f)$.

We conclude this section by using the results of Appendix A.4 to describe the homomorphism

$$
\mathrm{i}_{!}: H_{k}\left(\Lambda^{1}(M)\right) \rightarrow H_{k-n}\left(\Omega^{1}\left(M, q_{0}\right)\right),
$$

working with the same action functional $\mathbb{S}_{L}$ both on $\Lambda^{1}(M)$ and on $\Omega^{1}\left(M, q_{0}\right)$. Conditions (A-7) and (A-8) are implied by the assumption

$$
\gamma \in \mathscr{P}^{\Lambda}(L) \quad \Longrightarrow \quad \gamma(0) \neq q_{0},
$$


a condition holding for all but a countable set of $q_{0}$ 's. Under this assumption, up to the perturbation of the Morse-Smale pseudo-gradient vector fields $X_{L}^{\Lambda}$ and $X_{L}^{\Omega}$ for $\mathbb{S}_{L}^{\Lambda}$ and $\mathbb{S}_{L}^{\Omega}$, we may assume that the unstable manifold of each $\gamma_{1} \in \mathscr{P} \Lambda(L)$ is transverse to the stable manifold of each $\gamma_{2} \in \mathscr{P}^{\Omega}(L)$ in $\Lambda^{1}(M)$. When $i^{\Omega}\left(\gamma_{2}\right)=i^{\Lambda}\left(\gamma_{1}\right)-n$, the set

$$
W^{u}\left(\gamma_{1} ; X_{L}^{\Lambda}\right) \cap W^{s}\left(\gamma_{2} ; X_{L}^{\Omega}\right)
$$

consists of finitely many oriented points, which determine the integer $n_{\mathrm{i} !}\left(\gamma_{1}, \gamma_{2}\right)$. These integers are the coefficients of a chain map

$$
M \mathrm{i}_{!}: M_{*}\left(\mathbb{S}_{L}^{\Lambda}\right) \rightarrow M_{*-n}\left(\mathbb{S}_{L}^{\Omega}\right),
$$

which in homology induces the homomorphism i! .

\subsection{Morse description of the Pontrjagin product}

In the last section we have described the vertical arrows of diagram (1-1), as well as a preferred left inverse of the top-right vertical arrow. The top horizontal arrow is described at the end of Appendix A.4. It remains to describe the middle and the bottom horizontal arrows, that is the loop product and the Pontrjagin product. This section is devoted to the description of the Pontrjagin product, while in the next one we will deal with the loop product. The following Propositions 2.2, 2.4 and 2.5 are consequences of the general statements in the Appendix.

Given two Lagrangians $L_{1}, L_{2} \in C^{\infty}([0,1] \times T M)$ which satisfy (L0) ${ }^{\Omega}$, (L1), (L2), and are such that $L_{1}(1, \cdot)=L_{2}(0, \cdot)$ with all the time derivatives, we define a Lagrangian $L_{1} \# L_{2} \in C^{\infty}([0,1] \times T M)$ in the same class by setting

$$
L_{1} \# L_{2}(t, q, v)= \begin{cases}2 L_{1}(2 t, q, v / 2) & \text { if } 0 \leq t \leq 1 / 2, \\ 2 L_{2}(2 t-1, q, v / 2) & \text { if } 1 / 2 \leq t \leq 1 .\end{cases}
$$

The curve $\gamma:[0,1] \rightarrow M$ is a solution of the Euler-Lagrange equation (2-1) with $L=L_{1} \# L_{2}$ if and only if the rescaled curves $t \mapsto \gamma(t / 2)$ and $t \mapsto \gamma((t+1) / 2)$ solve the corresponding equation given by the Lagrangians $L_{1}$ and $L_{2}$, on $[0,1]$. We assume that also $L_{1} \# L_{2}$ satisfies $(\mathrm{L} 0)^{\Omega}$.

In view of the results of Appendix A.3, we wish to consider the functional $\mathbb{S}_{L_{1}}^{\Omega} \oplus \mathbb{S}_{L_{2}}^{\Omega}$ on $\Omega^{1}\left(M, q_{0}\right) \times \Omega^{1}\left(M, q_{0}\right)$,

$$
\mathbb{S}_{L_{1}}^{\Omega} \oplus \mathbb{S}_{L_{2}}^{\Omega}\left(\gamma_{1}, \gamma_{2}\right)=\mathbb{S}_{L_{1}}^{\Omega}\left(\gamma_{1}\right)+\mathbb{S}_{L_{2}}^{\Omega}\left(\gamma_{2}\right),
$$

and the functional $\mathbb{S}_{L_{1} \# L_{2}}^{\Omega}$ on $\Omega^{1}\left(M, q_{0}\right)$. The concatenation map

$$
\Gamma: \Omega^{1}\left(M, q_{0}\right) \times \Omega^{1}\left(M, q_{0}\right) \rightarrow \Omega^{1}\left(M, q_{0}\right)
$$


is nowhere a submersion, so condition (A-2) for the triplet $\left(\Gamma, \mathbb{S}_{L_{1}}^{\Omega} \oplus \mathbb{S}_{L_{2}}^{\Omega}, \mathbb{S}_{L_{1} \# L_{2}}^{\Omega}\right.$ ) requires that the image of $\Gamma$ does not meet the critical set of $\mathbb{S}_{L_{1} \# L_{2}}^{\Omega}$, that is

$$
\gamma \in \mathscr{P}^{\Omega}\left(L_{1} \# L_{2}\right) \quad \Longrightarrow \quad \gamma(1 / 2) \neq q_{0} .
$$

Notice that (2-9) allows $L_{1}$ and $L_{2}$ to be equal, and actually it allows them to be also autonomous (however, it implies that $q_{0}$ is not a stationary solution, so they cannot be the Lagrangian associated to a geodesic flow).

Assuming the generic condition (2-9), condition (A-3) is automatically fulfilled. Moreover, if $X_{L_{1}}^{\Omega}$ and $X_{L_{2}}^{\Omega}$ are pseudo-gradients for $\mathbb{S}_{L_{1}}^{\Omega}$ and $\mathbb{S}_{L_{2}}^{\Omega}$, we have that for every $\gamma_{1} \in \mathscr{P}^{\Omega}\left(L_{1}\right)$ and $\gamma_{2} \in \mathscr{P}^{\Omega}\left(L_{2}\right)$,

$$
\Gamma\left(W^{u}\left(\left(\gamma_{1}, \gamma_{2}\right) ; X_{L_{1}}^{\Omega} \oplus X_{L_{2}}^{\Omega}\right)\right) \cap \operatorname{crit}\left(\mathbb{S}_{L_{1} \# L_{2}}^{\Omega}\right)=\varnothing .
$$

By Remark A.3, there is no need to perturb the vector field $X_{L_{1}}^{\Omega} \oplus X_{L_{2}}^{\Omega}$ on $\Omega^{1}\left(M, q_{0}\right) \times$ $\Omega^{1}\left(M, q_{0}\right)$ to achieve transversality, and we arrive at the following description of the Pontrjagin product.

Let $X_{L_{1}}^{\Omega}, X_{L_{2}}^{\Omega}$, and $X_{L_{1} \# L_{2}}^{\Omega}$ be Morse-Smale pseudo-gradients for $\mathbb{S}_{L_{1}}^{\Omega}, \mathbb{S}_{L_{1}}^{\Omega}$, and $\mathbb{S}_{L_{1} \# L_{2}}^{\Omega}$, respectively. Fix an arbitrary orientation of the unstable manifolds of each critical point of $\mathbb{S}_{L_{1}}^{\Omega}, \mathbb{S}_{L_{2}}^{\Omega}, \mathbb{S}_{L_{1} \# L_{2}}^{\Omega}$. Up to the perturbation of $X_{L_{1} \# L_{2}}^{\Omega}$, we get that the restriction of $\Gamma$ to the unstable manifold

$$
W^{u}\left(\left(\gamma_{1}, \gamma_{2}\right) ; X_{L_{1}}^{\Omega} \oplus X_{L_{2}}^{\Omega}\right)=W^{u}\left(\gamma_{1} ; X_{L_{1}}^{\Omega}\right) \times W^{u}\left(\gamma_{2} ; X_{L_{2}}^{\Omega}\right)
$$

of every critical point $\left(\gamma_{1}, \gamma_{2}\right) \in \mathscr{P}^{\Omega}\left(L_{1}\right) \times \mathscr{P}^{\Omega}\left(L_{2}\right)$ is transverse to the stable manifold

$$
W^{s}\left(\gamma ; X_{L_{1} \# L_{2}}^{\Omega}\right)
$$

of each critical point of $\mathbb{S}_{L_{1} \# L_{2}}^{\Omega}$. When $i^{\Omega}(\gamma)=i^{\Omega}\left(\gamma_{1}\right)+i^{\Omega}\left(\gamma_{2}\right)$, the corresponding intersections

$$
\left\{\left(\alpha_{1}, \alpha_{2}\right) \in W^{u}\left(\gamma_{1} ; X_{L_{1}}^{\Omega}\right) \times W^{u}\left(\gamma_{2} ; X_{L_{2}}^{\Omega}\right) \mid \Gamma\left(\alpha_{1}, \alpha_{2}\right) \in W^{s}\left(\gamma ; X_{L_{1} \# L_{2}}^{\Omega}\right)\right\},
$$

is a finite set of oriented points. Let $n_{\#}\left(\gamma_{1}, \gamma_{2} ; \gamma\right)$ be the algebraic sum of these orientation signs.

Proposition 2.2 The homomorphism

$$
\begin{aligned}
M_{\#}: M_{j}\left(\mathbb{S}_{L_{1}}^{\Omega}\right) \otimes M_{k}\left(\mathbb{S}_{L_{2}}^{\Omega}\right) \rightarrow M_{j+k}\left(\mathbb{S}_{L_{1} \# L_{2}}^{\Omega}\right), \\
\gamma_{1} \otimes \gamma_{2} \mapsto \sum_{\substack{\gamma \in \mathscr{\rho} \Omega \\
i^{\Omega}(\gamma)=j+k}} n_{\#}\left(\gamma_{1}, \gamma_{2} ; \gamma\right) \gamma,
\end{aligned}
$$

is a chain map, and it induces the Pontrjagin product \# in homology. 
Remark 2.3 It is not necessary to consider the Lagrangian $L_{1} \# L_{2}$ on the target space of this homomorphism. One could actually work with any three Lagrangians (with the suitable nondegeneracy condition replacing (2-9)). The choice of dealing with two Lagrangians $L_{1}, L_{2}$ and their concatenation $L_{1} \# L_{2}$ is important to get energy estimates in Floer homology. We have made this choice also here mainly to see which kind of nondegeneracy condition one needs.

\subsection{Morse description of the loop product}

The loop product is slightly more complicated than the other homomorphisms considered so far, because it consists of a composition where two homomorphisms are nontrivial (that is, not just identifications) when read on the Morse homology groups, namely the Umkehr map associated to the submanifold $\Theta^{1}(M)$ of figure-8 loops, and the homomorphism induced by the concatenation map $\Gamma: \Theta^{1}(M) \rightarrow \Lambda^{1}(M)$. We shall describe these homomorphisms separately, and then we will show a compact description of their composition. See also Cohen and Schwarz [18], where these Morse theoretical descriptions are extended to more general graphs than the figure- 8 .

Let us start by describing the Umkehr map

$$
e_{!}: H_{k}\left(\Lambda^{1}(M) \times \Lambda^{1}(M)\right) \rightarrow H_{k-n}\left(\Theta^{1}(M)\right) .
$$

Let $L_{1}, L_{2} \in C^{\infty}(\mathbb{T} \times T M)$ be Lagrangians which satisfy (L0) ${ }^{\Lambda}$, (L1), (L2), and are such that $L_{1} \oplus L_{2}$ satisfies (L0) ${ }^{\Theta}$. Assume also

$$
\gamma_{1} \in \mathscr{P}^{\Lambda}\left(L_{1}\right), \gamma_{2} \in \mathscr{P}^{\Lambda}\left(L_{2}\right) \quad \Longrightarrow \quad \gamma_{1}(0) \neq \gamma_{2}(0) .
$$

Notice that this condition prevents $L_{1}$ from coinciding with $L_{2}$, but it holds for a generic pair $\left(L_{1}, L_{2}\right)$. We shall consider the functional $\mathbb{S}_{L_{1}}^{\Lambda} \oplus \mathbb{S}_{L_{2}}^{\Lambda}$ on $\Lambda^{1}(M) \times$ $\Lambda^{1}(M)$, and the functional $\mathbb{S}_{L_{1} \oplus L_{2}}^{\Theta}$ on $\Theta^{1}(M)$. Condition (2-10) implies that the unconstrained functional $\mathbb{S}_{L_{1}}^{\Lambda} \oplus \mathbb{S}_{L_{2}}^{\Lambda}$ has no critical points on $\Theta^{1}(M)$, so conditions (A-7) and (A-8) hold.

By the discussion of Appendix A.4, we can find smooth Morse-Smale pseudo-gradients $X_{L_{1} \oplus L_{2}}^{\Lambda}$ and $X_{L_{1} \oplus L_{2}}^{\Theta}$ for $\mathbb{S}_{L_{1}}^{\Lambda} \oplus \mathbb{S}_{L_{2}}^{\Lambda}$ and $\mathbb{S}_{L_{1} \oplus L_{2}}^{\Theta}$ respectively, such that the unstable manifold $W^{u}\left(\gamma^{-} ; X_{L_{1} \oplus L_{2}}^{\Lambda}\right)$ of every $\gamma^{-}=\left(\gamma_{1}^{-}, \gamma_{2}^{-}\right) \in \mathscr{P}^{\Lambda}\left(L_{1}\right) \times \mathscr{P} \Lambda\left(L_{2}\right)$ is transverse to $\Theta^{1}(M)$ and to the stable manifold $W^{s}\left(\gamma^{+} ; X_{L_{1} \oplus L_{2}}^{\Theta}\right)$ of every $\gamma^{+} \in$ $\mathscr{P}^{\Theta}\left(L_{1} \oplus L_{2}\right)$. Actually, it is convenient to assume that $X_{L_{1} \oplus L_{2}}^{\Lambda}$ is so close to $X_{L_{1}}^{\Lambda} \oplus X_{L_{2}}^{\Lambda}$ that the Morse complex of $\left(\mathbb{S}_{L_{1}}^{\Lambda} \oplus \mathbb{S}_{L_{2}}^{\Lambda}, X_{L_{1} \oplus L_{2}}^{\Lambda}\right)$ equals the Morse complex of $\left(\mathbb{S}_{L_{1}}^{\Lambda} \oplus \mathbb{S}_{L_{2}}^{\Lambda}, X_{L_{1}}^{\Lambda} \oplus X_{L_{2}}^{\Lambda}\right)$, and that $X_{L_{1}}^{\Lambda} \oplus L_{2}=X_{L_{1}}^{\Lambda} \oplus X_{L_{2}}^{\Lambda}$ up to order one at the critical points. 
Fix an arbitrary orientation of the unstable manifold of every critical point of $\mathbb{S}_{L_{1}}^{\Lambda}$, $\mathbb{S}_{L_{2}}^{\Lambda}$, and $\mathbb{S}_{L_{1} \oplus L_{2}}^{\Theta}$. By our assumptions on $X_{L_{1} \oplus L_{2}}^{\Lambda}$, also the unstable manifolds

$$
W^{u}\left(\gamma^{-} ; X_{L_{1} \oplus L_{2}}^{\Lambda}\right)
$$

are oriented. When $i^{\Theta}\left(\gamma^{+}\right)=i^{\Lambda}\left(\gamma_{1}^{-}\right)+i^{\Lambda}\left(\gamma_{2}^{-}\right)-n$, the intersection

$$
W^{u}\left(\gamma^{-} ; X_{L_{1} \oplus L_{2}}^{\Lambda}\right) \cap W^{s}\left(\gamma^{+} ; X_{L_{1} \oplus L_{2}}^{\Theta}\right)
$$

is a finite set of oriented points. If we denote by $n_{e}\left(\gamma^{-}, \gamma^{+}\right)$the algebraic sum of these orientation signs, we have the following:

Proposition 2.4 The homomorphism

$$
M_{k}\left(\mathbb{S}_{L_{1}}^{\Lambda} \oplus \mathbb{S}_{L_{2}}^{\Lambda}\right) \rightarrow M_{k-n}\left(\mathbb{S}_{L_{1} \oplus L_{2}}^{\Theta}\right), \quad \gamma^{-} \mapsto \sum_{\substack{\left.\left.\gamma^{+} \in \mathscr{P} \Theta \\ i L_{1}^{\Theta} \oplus \nu_{2}\right) \\ \gamma^{+}\right)=k-n}} n_{e_{!}}\left(\gamma^{-}, \gamma^{+}\right) \gamma^{+},
$$

is a chain map, and it induces the Umkehr map e! in homology.

By composing this homomorphism with the Morse theoretical version of the exterior homology product described in Appendix A.3, that is the isomorphism

$$
M_{j}\left(\mathbb{S}_{L_{1}}^{\Lambda}\right) \otimes M_{h}\left(\mathbb{S}_{L_{2}}^{\Lambda}\right) \rightarrow M_{j+h}\left(\mathbb{S}_{L_{1}}^{\Lambda} \oplus \mathbb{S}_{L_{2}}^{\Lambda}\right),
$$

we obtain the homomorphism

$$
M_{!}: M_{j}\left(\mathbb{S}_{L_{1}}^{\Lambda}\right) \otimes M_{h}\left(\mathbb{S}_{L_{2}}^{\Lambda}\right) \rightarrow M_{j+h-n}\left(\mathbb{S}_{L_{1} \oplus L_{2}}^{\Theta}\right) .
$$

Here we are using the fact that the Morse complexes of $\left(\mathbb{S}_{L_{1}}^{\Lambda} \oplus \mathbb{S}_{L_{2}}^{\Lambda}, X_{L_{1} \oplus L_{2}}^{\Lambda}\right)$ and $\left(\mathbb{S}_{L_{1}}^{\Lambda} \oplus \mathbb{S}_{L_{2}}^{\Lambda}, X_{L_{1}}^{\Lambda} \oplus X_{L_{2}}^{\Lambda}\right)$ coincide.

Let us describe the homomorphism

$$
\Gamma_{*}: H_{k}\left(\Theta^{1}(M)\right) \rightarrow H_{k}\left(\Lambda^{1}(M)\right),
$$

induced by the concatenation map $\Gamma$. Let $L_{1}, L_{2} \in C^{\infty}([0,1] \times T M)$ be Lagrangians which satisfy (L1), (L2), and are such that $L_{1}(1, \cdot)=L_{2}(0, \cdot)$ and $L_{2}(1, \cdot)=L_{1}(0, \cdot)$ with all the time derivatives. We assume that $L_{1} \oplus L_{2}$ satisfies (L0) ${ }^{\Theta}$ and that the time periodic Lagrangian $L_{1} \# L_{2}$ satisfies (L0) ${ }^{\Lambda}$. We would like to apply the results of Appendix A.2 to the functionals $\mathbb{S}_{L_{1} \oplus L_{2}}^{\Theta}$ on $\Theta^{1}(M)$ and $\mathbb{S}_{L_{1} \# L_{2}}^{\Lambda}$ on $\Lambda^{1}(M)$. The map $\Gamma: \Theta^{1}(M) \rightarrow \Lambda^{1}(M)$ is nowhere a submersion, so condition (A-2) for the triplet $\left(\Gamma, \mathbb{S}_{L_{1} \oplus L_{2}}^{\Theta}, \mathbb{S}_{L_{1} \# L_{2}}^{\Lambda}\right)$ requires that $\Gamma\left(\Theta^{1}(M)\right)$ does not contain critical points of $\mathbb{S}_{L_{1} \# L_{2}}^{\Lambda}$. The latter fact is equivalent to the condition

$$
\gamma \in \mathscr{P}^{\Lambda}\left(L_{1} \# L_{2}\right) \quad \Longrightarrow \quad \gamma(1 / 2) \neq \gamma(0)
$$


which holds for a generic pair $\left(L_{1}, L_{2}\right)$. Assuming (2-11), conditions (A-2) and (A-3) are automatically fulfilled. Therefore, the discussion of Appendix A.2 implies that we can find Morse-Smale pseudo-gradients $X_{L_{1} \oplus L_{2}}^{\Theta}$ and $X_{L_{1} \# L_{2}}^{\Lambda}$ for $\mathbb{S}_{L_{1} \oplus L_{2}}^{\Theta}$ and $\mathbb{S}_{L_{1} \# L_{2}}^{\Lambda}$, respectively, such that the restriction of $\Gamma$ to the unstable manifold

$$
W^{u}\left(\gamma^{-} ; X_{L_{1} \oplus L_{2}}^{\Theta}\right)
$$

of every critical point $\gamma^{-}=\left(\gamma_{1}^{-}, \gamma_{2}^{-}\right) \in \mathscr{P}^{\Theta}\left(L_{1} \oplus L_{2}\right)$ is transverse to the stable manifold

$$
W^{s}\left(\gamma^{+} ; X_{L_{1} \# L_{2}}^{\Lambda}\right)
$$

of every critical point $\gamma^{+} \in \mathscr{P}^{\Lambda}\left(L_{1} \# L_{2}\right)$. Fix arbitrary orientations for the unstable manifolds of every critical point of $\mathbb{S}_{L_{1} \oplus L_{2}}^{\Theta}$ and $\mathbb{S}_{L_{1} \# L_{2}}^{\Lambda}$. When $i^{\Lambda}\left(\gamma^{+}\right)=i^{\Theta}\left(\gamma^{-}\right)$, the intersection

$$
\left\{\left(\alpha_{1}, \alpha_{2}\right) \in W^{u}\left(\gamma^{-} ; X_{L_{1} \oplus L_{2}}^{\Theta}\right) \mid \Gamma\left(\alpha_{1}, \alpha_{2}\right) \in W^{s}\left(\gamma^{+} ; X_{L_{1} \# L_{2}}^{\Lambda}\right)\right\},
$$

is a finite set of oriented points. If we denote by $n_{\Gamma}\left(\gamma^{-}, \gamma^{+}\right)$the algebraic sum of these orientation signs, we have the following:

Proposition 2.5 The homomorphism

$$
M_{\Gamma}: M_{k}\left(\mathbb{S}_{L_{1} \oplus L_{2}}^{\Theta}\right) \rightarrow M_{k}\left(\mathbb{S}_{L_{1} \# L_{2}}^{\Lambda}\right), \quad \gamma^{-} \mapsto \sum_{\substack{\gamma^{+} \in \mathscr{P} \Lambda \\ i^{\Lambda}\left(\gamma^{+}\right)=k}} n_{\Gamma}\left(\gamma^{-}, \gamma^{+}\right) \gamma^{+},
$$

is a chain map, and it induces the homomorphism $\Gamma_{*}: H_{k}\left(\Theta^{1}(M)\right) \rightarrow H_{k}\left(\Lambda^{1}(M)\right)$ in homology.

Therefore, the composition $M_{\Gamma} \circ M_{!}$induces the loop product in homology.

We conclude this section by exhibiting a compact description of the loop product

$$
\text { o: } H_{j}\left(\Lambda^{1}(M)\right) \otimes H_{k}\left(\Lambda^{1}(M)\right) \rightarrow H_{j+k-n}\left(\Lambda^{1}(M)\right) \text {. }
$$

Since we are not going to use this description, we omit the proof. Let $L_{1}, L_{2} \in$ $C^{\infty}(\mathbb{T} \times T M)$ be Lagrangians which satisfy (L0) ${ }^{\Lambda}$, (L1), (L2), such that $L_{1}(0, \cdot)=$ $L_{2}(0, \cdot)$ with all time derivatives, and such that the concatenated Lagrangian $L_{1} \# L_{2}$ defined by (2-8) satisfies (L0) ${ }^{\Lambda}$. We also assume (2-10), noticing that this condition prevents $L_{1}$ from coinciding with $L_{2}$. Let $X_{L_{1} \oplus L_{2}}^{\Lambda}$ and $X_{L_{1} \# L_{2}}^{\Lambda}$ be Morse-Smale pseudo-gradients for $\mathbb{S}_{L_{1}}^{\Lambda} \oplus \mathbb{S}_{L_{2}}^{\Lambda}$ and $\mathbb{S}_{L_{1} \# L_{2}}^{\Lambda}$, respectively. By (2-10), the functional $\mathbb{S}_{L_{1} \oplus L_{2}}^{\Theta}$ has no critical points on $\Theta^{1}(M)$, so up to the perturbation of $X_{L_{1} \oplus L_{2}}^{\Lambda}$, we can assume that for every $\gamma_{1} \in \mathscr{P}^{\Lambda}\left(L_{1}\right), \gamma_{2} \in \mathscr{P}^{\Lambda}\left(L_{2}\right)$, the unstable manifold $W^{u}\left(\left(\gamma_{1}, \gamma_{2}\right) ; X_{L_{1} \oplus L_{2}}^{\Lambda}\right)$ is transverse to $\Theta^{1}(M)$. Moreover, assumption (2-10) implies 
that the image of $\Theta^{1}(M)$ by the concatenation map $\Gamma$ does not contain any critical point of $\mathbb{S}_{L_{1} \# L_{2}}^{\Lambda}$. Therefore, up to the perturbation of $X_{L_{1} \# L_{2}}^{\Lambda}$, we can assume that for every $\gamma_{1} \in \mathscr{P}^{\Lambda}\left(L_{1}\right), \gamma_{2} \in \mathscr{P} \Lambda\left(L_{2}\right)$, the restriction of $\Gamma$ to the submanifold

$$
W^{u}\left(\left(\gamma_{1}, \gamma_{2}\right) ; X_{L_{1} \oplus L_{2}}^{\Lambda}\right) \cap \Theta^{1}(M)
$$

is transverse to the stable manifold $W^{s}\left(\gamma ; X_{L_{1} \# L_{2}}^{\Lambda}\right)$ of each $\gamma \in \mathscr{P} \Lambda(L)$. In particular, when $i^{\Lambda}(\gamma)=i^{\Lambda}\left(\gamma_{1}\right)+i^{\Lambda}\left(\gamma_{2}\right)-n$, the submanifold

$$
\left\{\left(\alpha_{1}, \alpha_{2}\right) \in W^{u}\left(\left(\gamma_{1}, \gamma_{2}\right) ; X_{L_{1} \oplus L_{2}}^{\Lambda}\right) \cap \Theta^{1}(M) \mid \Gamma\left(\alpha_{1}, \alpha_{2}\right) \in W^{s}\left(\gamma ; X_{L_{1} \# L_{2}}^{\Lambda}\right)\right\}
$$

is a finite set of oriented points. We contend that if $n_{\circ}\left(\gamma_{1}, \gamma_{2} ; \gamma\right)$ denotes the algebraic sum of the corresponding orientation signs, the following holds:

Proposition 2.6 The homomorphism

$$
\begin{aligned}
M_{j}\left(\mathbb{S}_{L_{1}}^{\Lambda}\right) \otimes M_{k}\left(\mathbb{S}_{L_{2}}^{\Lambda}\right) \rightarrow M_{j+k-n}\left(\mathbb{S}_{L_{1} \# L_{2}}^{\Lambda}\right), \\
\gamma_{1} \otimes \gamma_{2} \mapsto \sum_{\substack{\gamma \in \mathscr{P} \Lambda \\
i^{\Lambda}(\gamma)=j+k-n}} n_{\circ}\left(\gamma_{1}, \gamma_{2} ; \gamma\right) \gamma,
\end{aligned}
$$

is a chain map and it induces the loop product in homology.

\section{Floer homologies on cotangent bundles and their ring struc- tures}

\subsection{Floer homology for nonlocal conormal boundary conditions}

In this section we recall the construction of Floer homology for Hamiltonian orbits on cotangent bundles with nonlocal conormal boundary conditions. This is the Hamiltonian version of the setting described in Section 2.1. See our papers [4] and [2] (with Portaluri) for detailed proofs.

Let $Q$ be a closed manifold (in our applications, $Q$ is either $M$ or $M \times M$ ), and let $R$ be a closed submanifold of $Q \times Q$. We shall often denote the elements of the cotangent bundle $T^{*} Q$ as pairs $(q, p)$, where $q \in Q$ and $p \in T_{q}^{*} Q$. Let $\omega=d p \wedge d q$ be the standard symplectic form on the manifold $T^{*} Q$, that is the differential of the Liouville form $\eta:=p d q$. Equivalently, the Liouville form $\eta$ can be defined by

$$
\eta(\zeta)=x(D \pi(x)[\zeta]) \quad \text { for } \zeta \in T_{x} T^{*} M, x \in T^{*} Q,
$$


where $\pi: T^{*} Q \rightarrow Q$ is the bundle projection. Let $Y=p \partial_{p}$ be the standard Liouville vector field on $T^{*} Q$, which is defined by the identity

$$
\omega(Y, \cdot)=\eta
$$

Consider the class of smooth Hamiltonians $H$ on $[0,1] \times T^{*} Q$ such that:

(H1) $D H(t, q, p)[Y]-H(t, q, p) \geq h_{0}|p|^{2}-h_{1}$,

$$
\left|\nabla_{q} H(t, q, p)\right| \leq h_{2}\left(1+|p|^{2}\right),\left|\nabla_{p} H(t, q, p)\right| \leq h_{2}(1+|p|)
$$

for every $(t, q, p)$, for some constants $h_{0}>0, h_{1} \in \mathbb{R}, h_{2}>0$. Here $\nabla_{q}$ and $\nabla_{p}$ denote the horizontal and vertical components of the gradient, with respect to a Riemannian metric on $Q$ and to the induced metric on $T^{*} Q$, and $|\cdot|$ denotes the norm associated to such a metric. The fact that $Q$ is compact easily implies that (H1) and (H2) do not depend on the choice of this metric. Condition (H1) essentially says that $H$ grows at least quadratically in $p$ on each fiber of $T^{*} M$, and that it is radially convex for $|p|$ large. Condition (H2) implies that $H$ grows at most quadratically in $p$ on each fiber. Notice also that if $H$ is the Fenchel transform of a fiber-wise strictly convex Lagrangian $L$ in $C^{\infty}([0,1] \times T M)$ (see Section 4.1), then the term $D H(t, q, p)[Y(q, p)]-H(t, q, p)$ appearing in (H1) coincides with $L\left(t, q, D_{p} H(t, q, p)\right)$.

Let $X_{H}$ be the time-dependent Hamiltonian vector field associated to $H$ by the formula $\omega\left(X_{H}, \cdot\right)=-D_{x} H$. Condition (H2) implies the quadratic bound

$$
\left|X_{H}(t, q, p)\right| \leq h_{3}\left(1+|p|^{2}\right),
$$

for some $h_{3}>0$. Let $(t, x) \mapsto \phi^{H}(t, x)$ be the nonautonomous flow associated to the vector field $X_{H}$.

The conormal bundle $N^{*} R$ of $R$ in $Q \times Q$ is the set of covectors $x=(q, p)$ in $T^{*}(Q \times Q)$ such that $q=\left(q_{1}, q_{2}\right)$ belongs to $R$ and $p=\left(p_{1}, p_{2}\right) \in T_{\left(q_{1}, q_{2}\right)}^{*}(Q \times Q)$ vanishes identically on $T_{\left(q_{1}, q_{2}\right)} R$. It is a vector bundle over $R$ and the dimension of its fibers equals the codimension of $R$ in $Q \times Q$. The Liouville form of $T^{*}(Q \times Q)$ vanishes on $N^{*} R$, so in particular $N^{*} R$ is a Lagrangian submanifold of $T^{*}(Q \times Q)$. Actually, conormal bundles can be characterized as those middle dimensional closed submanifolds of a cotangent bundle on which the Liouville form vanishes; see Proposition 2.1 in [2].

We are interested in the set $\mathscr{P}^{R}(H)$ of solutions $x:[0,1] \rightarrow T^{*} Q$ of the Hamiltonian equation

$$
x^{\prime}(t)=X_{H}(t, x(t)),
$$


which satisfy the boundary conditions

$$
(x(0), \mathscr{b} x(1)) \in N^{*} R,
$$

where $\mathscr{C}$ is the antisymplectic involution

$$
\mathscr{C}: T^{*} Q \rightarrow T^{*} Q, \quad(q, p) \mapsto(q,-p) .
$$

Equivalently, we are looking at the intersections of the Lagrangian submanifold $N^{*} R$ with the Lagrangian submanifold given by the graph of $\mathscr{b} \circ \phi^{H}(1, \cdot)$ (we are always considering the standard symplectic form $\omega \oplus \omega$ on $T^{*}(Q \times Q)$, not the flipped one $\omega \oplus(-\omega)$, so a diffeomorphism $\varphi: T^{*} Q \rightarrow T^{*} Q$ is symplectic if and only if the graph of the composition $\mathscr{C} \circ \varphi$ is Lagrangian).

We assume that:

(H0) All the elements of $\mathscr{P}^{R}(H)$ are nondegenerate, meaning that the submanifolds $N^{*} R$ and graph $\mathscr{b} \circ \phi^{H}(1, \cdot)$ intersect transversally.

Given $x \in \mathscr{P}^{R}(H)$, we can conjugate the differential of the flow $D_{x} \phi^{H}(t, x(t))$ by a symplectic trivialization $\Psi$ of $x^{*}\left(T T^{*} Q\right)$ and we obtain a path

$$
G^{\Psi}:[0,1] \rightarrow \operatorname{Sp}(2 m), \quad m:=\operatorname{dim} Q,
$$

of symplectic automorphisms of $T^{*} \mathbb{R}^{m}$, endowed with its standard symplectic structure.

We assume that the symplectic trivialization $\Psi$ is vertical-preserving, meaning that it maps the vertical subbundle $T^{v} T^{*} M:=\operatorname{ker} D \pi$ into the vertical space $N^{*}(0)=$ $(0) \times\left(\mathbb{R}^{m}\right)^{*} \subset T^{*} \mathbb{R}^{m}$, and has the property that the following linear subspace of $T^{*} \mathbb{R}^{m} \times T^{*} \mathbb{R}^{m}=T^{*} \mathbb{R}^{2 m}$,

$$
\left(\Psi(0) \times C \Psi(1) D^{\mathscr{C}}(\mathscr{b} x(1))\right) T_{(x(0), \mathscr{C} x(1))} N^{*} R,
$$

is the conormal space $N^{*} W^{\Psi}$ of some linear subspace $W^{\Psi} \subset \mathbb{R}^{m} \times \mathbb{R}^{m}$, where $C$ is the antisymplectic involution of $T^{*} \mathbb{R}^{m}$ which maps $(q, p)$ into $(q,-p)$. Then the Maslov index of $x$ with respect to the nonlocal boundary condition induced by $R$ is defined as

$$
\mu^{R}(x):=\mu\left(\operatorname{graph} G^{\Psi} C, N^{*} W^{\Psi}\right)+\frac{1}{2}(\operatorname{dim} R-\operatorname{dim} Q),
$$

where $\mu$ denotes the relative Maslov index of two paths of Lagrangian subspaces of $T^{*} \mathbb{R}^{2 m}$, in the sense of [42] (see also Section 5.1 for sign conventions). The fact that $x$ is nondegenerate implies that $\mu^{R}(x)$ is an integer, and the assumptions on the trivialization $\Psi$ imply that this integer does not depend on the choice of $\Psi$ (see 
Proposition 3.3 in [2]). The normalizing constant in the definition above is chosen in such a way that, when $H$ is the Fenchel dual of a Lagrangian $L, \mu^{R}(x)$ coincides with the Morse index of the corresponding solution of the Euler-Lagrange equation associated to $L$ (see Section 4.1).

The elements of $\mathscr{P}^{R}(H)$ are the critical points of the Hamiltonian action functional

$$
\mathbb{A}_{H}(x)=\int_{[0,1]} x^{*}(\eta-H d t)=\int_{0}^{1}\left(p(t)\left[q^{\prime}(t)\right]-H(t, q(t), p(t))\right) d t,
$$

on the space of curves $x:[0,1] \rightarrow T^{*} Q$ which satisfy (3-4). Indeed, the differential of $\mathbb{A}_{H}$ on the space of free paths on $T^{*} Q$ is

$$
D \mathbb{A}_{H}(x)[\zeta]=\int_{0}^{1} \omega\left(\zeta, x^{\prime}-X_{H}(t, x)\right) d t+\eta(x(1))[\zeta(1)]-\eta(x(0))[\zeta(0)],
$$

and the boundary term vanishes when $x$ satisfies (3-4) and the variation $\zeta$ satisfies

$$
\left(\zeta(0), D^{\mathscr{C}}(x(1))[\zeta(1)]\right) \in T_{(x(0), \mathscr{C} x(1))} N^{*} R,
$$

because the Liouville form is zero on conormal bundles. The nondegeneracy assumption, together with conditions (H1) and (H2), imply that the set of $x \in \mathscr{P}^{R}(H)$ with $\mathbb{A}_{H}(x) \leq A$ is finite, for every $A \in \mathbb{R}$. Indeed, this follows immediately from the following general:

Lemma 3.1 Let $H \in C^{\infty}\left([0,1] \times T^{*} Q\right)$ be a Hamiltonian satisfying (H1) and (H2). For every $A \in \mathbb{R}$ there exists a compact subset $K \subset T^{*} Q$ such that each orbit $x:[0,1] \rightarrow$ $T^{*} Q$ of $X_{H}$ with $\mathbb{A}_{H}(x) \leq A$ lies in $K$.

Proof Let $x=(q, p)$ be an orbit of $X_{H}$ such that $\mathbb{A}_{H}(x) \leq A$. Since $x$ is an orbit of $X_{H}$, by (3-1),

$$
\eta(x)\left[x^{\prime}\right]-H(t, x)=\omega\left(Y(x), X_{H}(t, x)\right)-H(t, x)=D H(t, x)[Y(x)]-H(t, x) .
$$

Therefore (H1) implies that $|p|$ is uniformly bounded in $L^{2}([0,1])$. By $(3-2),\left|x^{\prime}\right|$ is uniformly bounded in $L^{1}([0,1])$. Therefore $x$ is uniformly bounded in $W^{1,1}$, hence in $L^{\infty}$.

Remark 3.2 Assume that the flow generated by a Hamiltonian $H \in C^{\infty}\left([0,1] \times T^{*} Q\right)$ is globally defined (for instance, this holds if $H$ is coercive and $\left|\partial_{t} H\right| \leq c(|H|+1)$ ). Then the conclusion of Lemma 3.1 holds assuming just that the function $D H[Y]-H$ is coercive (a much weaker assumption than (H1), still implying that $H$ is coercive), without any upper bound such as $(\mathrm{H} 2)$. 
Let us fix a Riemannian metric $\langle\cdot, \cdot\rangle$ on $Q$. This metric induces metrics on $T Q$ and on $T^{*} Q$, both denoted by $\langle\cdot, \cdot\rangle$. It induces also an identification $T^{*} Q \cong T Q$, horizontal-vertical splittings of both $T T Q$ and $T T^{*} Q$, and a particular almost complex structure $J$ on $T^{*} M$, namely the one which in the horizontal-vertical splitting takes the form

$$
J=\left(\begin{array}{cc}
0 & -I \\
I & 0
\end{array}\right) .
$$

This almost complex structure is $\omega$-compatible, meaning that

$$
\langle\xi, \eta\rangle=\omega(J \xi, \eta), \quad \forall \xi, \eta \in T_{x} T^{*} Q, \forall x \in T^{*} Q .
$$

Notice that our sign convention here differs from the one used in [4]. The reason is that here we prefer to see the leading term in the Floer equation as a Cauchy-Riemann operator, and not as an anti-Cauchy-Riemann operator.

The $L^{2}$-negative gradient equation for the Hamiltonian action functional $\mathbb{A}_{H}$ is the Floer equation

$$
\bar{\partial}_{J, H}(u):=\partial_{s} u+J(u)\left[\partial_{t} u-X_{H}(t, u)\right]=0,
$$

for $u=u(s, t),(s, t) \in \mathbb{R} \times[0,1]$. A generic choice of the Hamiltonian $H$ makes the following space of solutions of the Floer equation (3-8) with nonlocal conormal boundary conditions defined by $R$,

$$
\begin{aligned}
& \mu_{\partial}^{R}(x, y)=\left\{u \in C^{\infty}\left(\mathbb{R} \times[0,1], T^{*} Q\right) \mid(u(s, 0), \mathscr{b} u(s, 1)) \in N^{*} R \forall s \in \mathbb{R},\right. \\
& \left.\bar{\partial}_{J, H}(u)=0, \text { and } \lim _{s \rightarrow-\infty} u(s, t)=x(t), \lim _{s \rightarrow+\infty} u(s, t)=y(t)\right\}
\end{aligned}
$$

a manifold of dimension $\mu^{R}(x)-\mu^{R}(y)$, for every $x, y \in \mathscr{P}^{R}(H)$. Here generic means for a countable intersection of open and dense subsets of the space of smooth time-dependent Hamiltonians satisfying ( $\mathrm{H} 0),(\mathrm{H} 1)$ and $(\mathrm{H} 2)$, with respect to suitable topologies (we refer to Floer, Hofer and Salamon [26] for transversality issues). In particular, the perturbation of a given Hamiltonian $H$ satisfying (H0), (H1), (H2) can be chosen in such a way that the discrete set $\mathscr{P}^{R}(H)$ is unaffected.

Remark 3.3 As it is well-known, transversality can also be achieved for a fixed Hamiltonian by perturbing the almost complex structure $J$ in a time-dependent way. In order to have good compactness properties for the spaces $\mu_{\partial}^{R}$ one needs the perturbed almost complex structure $J_{1}$ to be $C^{0}$-close enough to the metric one $J$ defined by (3-7) (see [4, Theorem 1.14]). Other compactness issues in this paper would impose further restrictions on the distance between $J_{1}$ and $J$. For this reason here we prefer 
to work with the fixed almost complex structure $J$, and to achieve transversality by perturbing the Hamiltonian. A different approach would be to choose almost complex structures on $T^{*} Q$ which are of contact type on $T^{*} Q \backslash Q$, seen as the symplectization of the unit cotangent sphere bundle (see eg Viterbo [52]). In this case, compactness of the spaces $M_{\partial}^{R}$ follows from the maximum principle, but one needs more restrictive assumptions on the behavior of the Hamiltonian $H$ for $|p|$ large.

The manifolds $\mu_{\partial}^{R}(x, y)$ can be oriented in a coherent way. Assumptions (H1) and (H2) imply that these manifolds have nice compactifications. In particular, when $\mu^{R}(x)-\mu^{R}(y)=1, \mu_{\partial}^{R}(x, y)$ consists of finitely many one-parameter families of solutions $\sigma \mapsto u(\cdot+\sigma, \cdot)$, each of which comes with a sign \pm 1 , depending whether its orientation agrees or not with the orientation determined by letting $\sigma$ increase. The algebraic sum of these numbers is an integer $n_{\partial}^{R}(x, y)$. If we let $F_{k}^{R}(H)$ denote the free abelian group generated by the elements $x \in \mathscr{P}^{R}(H)$ of index $\mu^{R}(x)=k$, the above coefficients define the homomorphism

$$
\partial: F_{k}^{R}(H) \rightarrow F_{k-1}^{R}(H), \quad x \mapsto \sum_{\substack{y \in \mathscr{P} R \\ \mu^{R}(y)=k-1}} n_{\partial}(x, y) y,
$$

which turns out to be a boundary operator. The resulting chain complex $F_{*}^{R}(H)$ is the Floer complex associated to the Hamiltonian $H$ and to the nonlocal conormal boundary conditions defined by $R$. If we change the metric on $Q$ - hence the almost complex structure $J$ - and the orientation data, the Floer complex $F_{*}^{R}(H)$ changes by an isomorphism. If we change the Hamiltonian $H$, the new Floer complex is homotopically equivalent to the old one. In particular, the homology of the Floer complex does not depend on the metric, on $H$, and on the orientation data. This fact allows us to denote this graded abelian group as $H F_{*}^{R}\left(T^{*} Q\right)$, and to call it the Floer homology of $T^{*} Q$ with nonlocal conormal boundary conditions defined by $R$.

As in Section 2.1, here we are interested in the following three boundary conditions.

Periodic boundary conditions Here $Q=M$ is closed and oriented, and $R=\Delta_{M}$ is the diagonal in $M \times M$. Under the extra assumption that $H$ extends as a smooth 1-periodic function on $\mathbb{R} \times T M$, the set $\mathscr{P}^{\Delta_{M}}(H)$ is precisely the set of 1 -periodic solutions of the Hamiltonian equation (3-3). We also use the notation $\mathscr{P} \Lambda(H):=$ $\mathscr{P}^{\Delta_{M}}(H)$. The nondegeneracy condition (H0) is just:

$(\mathrm{H} 0)^{\Lambda}$ For every $x \in \mathscr{P}^{\Lambda}(H)$, the number 1 is not an eigenvalue of

$$
D_{x} \phi^{H}(1, x(0)): T_{x(0)} T^{*} M \rightarrow T_{x(0)} T^{*} M .
$$


The Maslov index $\mu^{\Delta_{M}}(x)$ coincides with the Conley-Zehnder index of the periodic orbit $x$, that we denote also by $\mu^{\Lambda}(x)$ (notice that the normalizing constant in (3-5) vanishes). In the definition of such an index, one can choose the trivialization of $x^{*}\left(T T^{*} M\right)$ to be any 1-periodic symplectic vertical-preserving trivialization. If $M$ is not orientable and the closed curve $x$ is orientation-reversing, there are no 1-periodic and vertical-preserving trivializations of $x^{*}\left(T T^{*} M\right)$, so one has either to give up the periodicity, as in definition (3-5), or the preservation of the vertical subbundle, as in [53]. Floer homology with periodic boundary conditions is defined (with integer coefficients) also for nonorientable manifolds, but since in this paper we are interested in the loop product with integer coefficients, we assume orientability. The elements $u$ of $\mu_{\partial}^{\Lambda}(x, y):=\mu_{\partial}^{\Delta_{M}}(x, y)$ are actually smooth solutions of the Floer equation (3-8) on the cylinder, that is

$$
u: \mathbb{R} \times \mathbb{T} \rightarrow T^{*} M .
$$

The corresponding Floer complex is also denoted by $F_{*}^{\Lambda}(H)$, and its homology by $H F_{*}^{\Lambda}\left(T^{*} M\right)$.

Dirichlet boundary conditions Here $Q=M$ and $R$ consists of the single point $\left(q_{0}, q_{0}\right)$, for some fixed $q_{0} \in M$. The set $\mathscr{P}^{\left(q_{0}, q_{0}\right)}(H)$ is precisely the set of solutions $x:[0,1] \rightarrow T^{*} M$ of the Hamiltonian equation (3-3) such that $\pi \circ x(0)=\pi \circ x(1)=q_{0}$, and we denote such a set also by $\mathscr{P}^{\Omega}(H)$. The nondegeneracy condition is now:

$(\mathrm{H} 0)^{\Omega} \quad$ For every $x \in \mathscr{P}^{\Omega}(H)$, the linear mapping $D_{x} \phi^{H}(1, x(0)): T_{x(0)} T^{*} M \rightarrow$ $T_{x(1)} T^{*} M$ maps the vertical subspace $T_{x(0)}^{v} T^{*} M$ at $x(0)$ into a subspace having intersection (0) with the vertical subspace $T_{x(1)}^{v} T^{*} M$ at $x(1)$.

The Maslov index $\mu^{\left(q_{0}, q_{0}\right)}(x)$, that we denote also by $\mu^{\Omega}(x)$, is just the relative Maslov index of the path of Lagrangian subspaces $D \phi^{H}(t, x(0)) T_{x(0)}^{v} T^{*} M-$ transported into $T^{*} \mathbb{R}^{n}$ by means of a vertical-preserving symplectic trivialization - with respect to the vertical space $N^{*}(0)=(0) \times\left(\mathbb{R}^{n}\right)^{*}$. The boundary condition for the elements $u$ of $\mu_{\partial}^{\Omega}(x, y):=\mu_{\partial}^{\left(q_{0}, q_{0}\right)}(x, y)$ is the local condition

$$
u(s, 0) \in T_{q_{0}}^{*} M, \quad u(s, 1) \in T_{q_{0}}^{*} M, \quad \forall s \in \mathbb{R} .
$$

The corresponding Floer complex is also denoted by $F_{*}^{\Omega}(H)$, and its homology by $H F_{*}^{\Omega}\left(T^{*} M\right)$.

Figure- 8 boundary conditions Here $Q=M \times M$ and $R=\Delta_{M}^{(4)}$ is the fourth diagonal in $M^{4}$ :

$$
\Delta_{M}^{(4)}:=\{(q, q, q, q) \mid q \in M\}
$$


In this case, it is convenient (although not necessary) to chose $H$ of the form $H=$ $H_{1} \oplus H_{2}$, where

$H_{1} \oplus H_{2}(t, x):=H_{1}\left(t, x_{1}\right)+H_{2}\left(t, x_{2}\right), \quad \forall t \in[0,1], \forall x=\left(x_{1}, x_{2}\right) \in T^{*} M \times T^{*} M$, so that the elements of $\mathscr{P}^{(4)}(H)$ are the pairs of $T^{*} M$-valued curves $\left(x_{1}, x_{2}\right)$ such that each $x_{j}$ solves the Hamiltonian equation induced by $H_{j}$, and such that the coupling boundary conditions

$\pi \circ x_{1}(0)=\pi \circ x_{2}(0)=\pi \circ x_{1}(1)=\pi \circ x_{2}(1), \quad x_{1}(1)-x_{1}(0)+x_{2}(1)-x_{2}(0)=0$, hold. The set of $\mathscr{P}_{M}^{(4)}\left(H_{1} \oplus H_{2}\right)$ is also denoted by $\mathscr{P}^{\Theta}\left(H_{1} \oplus H_{2}\right)$. The corresponding nondegeneracy condition is:

$(\mathrm{H} 0)^{\Theta}$ Every solution $x=\left(x_{1}, x_{2}\right) \in \mathscr{P}^{\Theta}\left(H_{1} \oplus H_{2}\right)$ is nondegenerate, meaning that the graph of the map $\mathscr{b} \circ \phi^{H_{1} \oplus H_{2}}(1, \cdot)$ is transverse to the submanifold $N^{*} \Delta_{M}^{(4)}$ at the point $(x(0), \mathscr{C} x(1))$.

If $x=\left(x_{1}, x_{2}\right) \in \mathscr{P}^{\Theta}\left(H_{1} \oplus H_{2}\right)$, the Maslov index $\mu^{\Delta_{M}^{(4)}}(x)$, that we denote simply by $\mu^{\Theta}(x)$, is the integer

$$
\mu^{\Theta}(x)=\mu\left(\operatorname{graph} G^{\Psi} C, \Delta_{\mathbb{R}^{n}}^{(4)}\right)-n / 2,
$$

where the symplectic path $G^{\Psi}:[0,1] \rightarrow \mathrm{Sp}(4 n)$ is obtained by conjugating the differential of the flow $\phi^{H_{1} \oplus H_{2}}$ along $x$ by a trivialization $\Psi$ of $x^{*}\left(T T^{*} M^{2}\right)$ of the form $\Psi=\Psi_{1} \oplus \Psi_{2}$, where each $\Psi_{j}$ is the canonical vertical-preserving symplectic trivialization of $x_{j}^{*}\left(T T^{*} M\right)$ induced by a trivialization of $\left(\pi \circ x_{j}\right)^{*}(T M)$ over the circle $\mathbb{T}$. The boundary condition for the elements $u$ of $\mu_{\partial}^{\Theta}(x, y):=M_{\partial}^{\Delta_{M}^{(4)}}(x, y)$ is the nonlocal Lagrangian boundary condition

$$
(u(s, 0), \mathscr{C} u(s, 1)) \in N^{*} \Delta_{M}^{(4)}, \quad \forall s \in \mathbb{R} .
$$

The corresponding Floer complex is also denoted by $F_{*}^{\Theta}(H)$, and its homology by $H F_{*}^{\Theta}\left(T^{*} M\right)$.

\subsection{The Floer equation on triangles and pair-of-pants}

Additional algebraic structures on Floer homology are defined by extending the Floer equation to more general Riemann surfaces than the strip $\mathbb{R} \times[0,1]$ and the cylinder $\mathbb{R} \times \mathbb{T}$.

Let $(\Sigma, j)$ be a Riemann surface, possibly with boundary. For $u \in C^{\infty}\left(\Sigma, T^{*} M\right)$ consider the nonlinear Cauchy-Riemann operator

$$
\bar{D}_{J} u=\frac{1}{2}(D u+J(u) \circ D u \circ j),
$$


that is the complex antilinear part of $D u$ with respect to the almost-complex structure $J$. The operator $\bar{D}_{J}$ is a section of the bundle over $C^{\infty}\left(\Sigma, T^{*} M\right)$ whose fiber at $u$ is $\Omega^{0,1}\left(\Sigma, u^{*}\left(T T^{*} M\right)\right)$, the space of antilinear one-forms on $\Sigma$ taking values in the vector bundle $u^{*}\left(T T^{*} M\right)$. If we choose a holomorphic coordinate $z=s+i t$ on $\Sigma$, the operator $\bar{D}_{J}$ takes the form

$$
\bar{D}_{J} u=\frac{1}{2}\left(\partial_{s} u+J(u) \partial_{t} u\right) d s-\frac{1}{2} J(u)\left(\partial_{s} u+J(u) \partial_{t} u\right) d t .
$$

This expression shows that the leading term $\bar{\partial}_{J}:=\partial_{s}+J(\cdot) \partial_{t}$ in the Floer equation (3-8) can be extended to arbitrary Riemann surfaces, at the only cost of considering an equation which does not take values on a space of tangent vector fields, but on a space of antilinear one-forms.

When $\Sigma$ has a global coordinate $z=s+i t$, as in the case of the strip $\mathbb{R} \times[0,1]$ or of the cylinder $\mathbb{R} \times \mathbb{T}$, we can associate to the Hamiltonian term in the Floer equation the complex antilinear one-form

$$
F_{J, H}(u)=-\frac{1}{2}\left(J(u) X_{H}(t, u) d s+X_{H}(t, u) d t\right) \in \Omega^{0,1}\left(\Sigma, u^{*}\left(T T^{*} M\right)\right) .
$$

Formula (3-10) shows that the Floer equation (3-8) is equivalent to

$$
\bar{D}_{J} u+F_{J, H}(u)=0 .
$$

If we wish to use the formulation (3-12) to extend the Floer equation to more general Riemann surfaces, we encounter the difficulty that - unlike $\bar{D}_{J}-$ the Hamiltonian term $F_{J, H}$ is defined in terms of coordinates.

One way to get around this difficulty is to consider Riemann surfaces with cylindrical or strip-like ends, each of which is endowed with some fixed holomorphic coordinate $z=s+i t$, to define the operator $F_{J, H}$ on such ends, and then to extend it to the whole $\Sigma$ by considering a Hamiltonian $H$ which also depends on $s$ and vanishes far from the ends. In this way, only the Cauchy-Riemann operator acts in the region far from the ends. This approach is adopted in $[46 ; 39 ; 38]$.

A drawback of this method is that one loses sharp energy identities relating some norm of $u$ to the jump of the Hamiltonian action functional. Moreover, an $s$-dependent Hamiltonian which vanishes for some values of $s$ cannot satisfy assumptions (H1) and (H2). These facts lead to problems with compactness when dealing - as we are here with a noncompact symplectic manifold.

Therefore, we shall use a different method to extend the Hamiltonian term $F_{J, H}$. We shall describe this construction in the case of the triangle and the pair-of-pants surface, although the same idea could be generalized to any Riemann surface. 
Let $\Sigma_{\Upsilon}^{\Omega}$ be the holomorphic triangle, that is the Riemann surface consisting of a closed triangle with the three vertices removed (equivalently, a closed disk with three boundary points removed). Let $\Sigma_{\Upsilon}^{\Lambda}$ be the pair-of-pants Riemann surface, that is the sphere with three points removed.

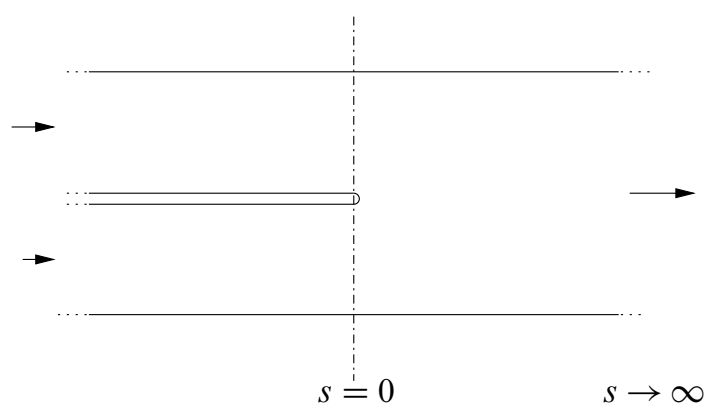

Figure 1: The strip with a slit $\Sigma_{\Upsilon}^{\Omega}$

The Riemann surface $\Sigma_{\Upsilon}^{\Omega}$ can be described as a strip with a slit: One takes the disjoint union

$$
\mathbb{R} \times[-1,0] \sqcup \mathbb{R} \times[0,1]
$$

and identifies $\left(s, 0^{-}\right)$with $\left(s, 0^{+}\right)$for every $s \geq 0$. See Figure 1 . The resulting object is indeed a Riemann surface with interior

$$
\left.\left.\operatorname{Int}\left(\Sigma_{\Upsilon}\right)=(\mathbb{R} \times]-1,1[) \backslash(]-\infty, 0\right] \times\{0\}\right)
$$

endowed with the complex structure of a subset of $\mathbb{R}^{2} \cong \mathbb{C},(s, t) \mapsto s+i t$, and three boundary components

$$
\mathbb{R} \times\{-1\}, \quad \mathbb{R} \times\{1\}, \quad]-\infty, 0] \times\left\{0^{-}, 0^{+}\right\} .
$$

The complex structure at each boundary point other than $0=(0,0)$ is induced by the inclusion in $\mathbb{C}$, whereas a holomorphic coordinate at 0 is given by the map

$$
\{\zeta \in \mathbb{C}|\operatorname{Re} \zeta \geq 0,| \zeta \mid<1\} \rightarrow \Sigma_{\Upsilon}^{\Omega}, \quad \zeta \mapsto \zeta^{2},
$$

which maps the boundary line $\{\operatorname{Re} \zeta=0,|\zeta|<1\}$ into the portion of the boundary ]$-1,0] \times\left\{0^{-}, 0^{+}\right\}$.

Similarly, the pair-of-pants $\Sigma_{\Upsilon}^{\Lambda}$ can be described as the following quotient of a strip with a slit: In the disjoint union $\mathbb{R} \times[-1,0] \sqcup \mathbb{R} \times[0,1]$ we consider the identifications

$$
\begin{array}{ll}
(s,-1) \sim\left(s, 0^{-}\right) \\
\left(s, 0^{+}\right) \sim(s, 1)
\end{array} \quad \text { for } s \leq 0, \quad \begin{aligned}
& \left(s, 0^{-}\right) \sim\left(s, 0^{+}\right) \\
& (s,-1) \sim(s, 1)
\end{aligned} \quad \text { for } s \geq 0 .
$$

See Figure 2. 


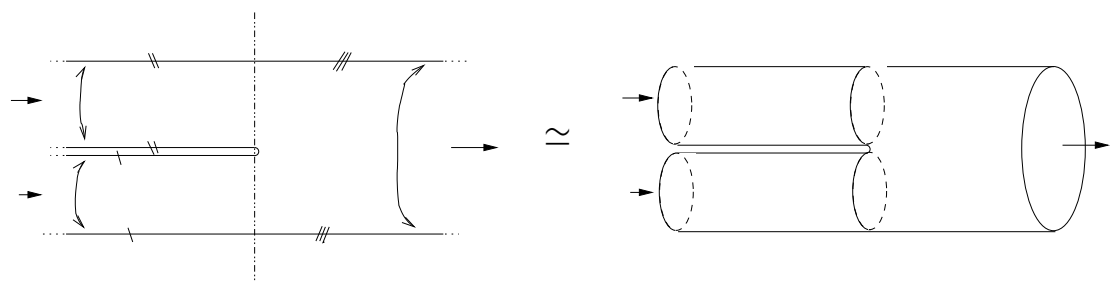

Figure 2: The pair-of-pants $\Sigma_{\Upsilon}^{\Lambda}$

This object is a Riemann surface without boundary, by considering the standard complex structure at every point other than $(0,0) \sim(0,-1) \sim(0,1)$, and by choosing the holomorphic coordinate

$$
\{\zeta \in \mathbb{C}|| \zeta \mid<1 / \sqrt{2}\} \rightarrow \Sigma_{\Upsilon}^{\Lambda}, \quad \zeta \mapsto \begin{cases}\zeta^{2} & \text { if } \operatorname{Re} \zeta \geq 0, \\ \zeta^{2}+i & \text { if } \operatorname{Re} \zeta \leq 0, \operatorname{Im} \zeta \geq 0, \\ \zeta^{2}-i & \text { if } \operatorname{Re} \zeta \leq 0, \operatorname{Im} \zeta \leq 0,\end{cases}
$$

at this point.

The advantage of these representations is that now $\Sigma_{\Upsilon}^{\Omega}$ and $\Sigma_{\Upsilon}^{\Lambda}$ are endowed with a global coordinate $z=s+i t$, which is holomorphic everywhere except at the point $(0,0)$ (identified with $(0,-1)$ and $(0,1)$ in the $\Lambda$ case). We refer to such a point as the singular point: it is a regular point for the complex structure of $\Sigma_{\Upsilon}^{\Omega}$ or $\Sigma_{\Upsilon}^{\Lambda}$, but it is singular for the global coordinate $z=s+i t$. In fact, the canonical map

$$
\Sigma_{\Upsilon}^{\Lambda} \rightarrow \mathbb{R} \times \mathbb{T}, \quad(s, t) \mapsto(s, t),
$$

is a $2: 1$ branched covering of the cylinder.

Let $H \in C^{\infty}\left([-1,1] \times T^{*} M\right)$. If $u \in C^{\infty}\left(\Sigma_{\Upsilon}^{\Omega}, T^{*} M\right)$, the complex antilinear oneform $F_{J, H}(u)$ is everywhere defined by equation (3-11). We just need to check the regularity of $F_{J, H}(u)$ at the singular point. Writing $F_{J, H}(u)$ in terms of the holomorphic coordinate $\zeta=\sigma+i \tau$ by means of (3-13), we find

$$
F_{J, H}(u)=(\tau I-\sigma J(u)) X_{H}(2 \sigma \tau, u) d \sigma+(\sigma I+\tau J(u)) X_{H}(2 \sigma \tau, u) d \tau .
$$

Therefore, $F_{J, H}(u)$ is smooth, and actually it vanishes at the singular point.

Assume now that $H \in C^{\infty}\left(\mathbb{R} / 2 \mathbb{Z} \times T^{*} M\right)$ is such that $H(-1, \cdot)=H(0, \cdot)=H(1, \cdot)$ with all the time derivatives. If $u \in C^{\infty}\left(\Sigma_{\Upsilon}^{\Lambda}, T^{*} M\right)$, (3-11) defines a smooth complex antilinear one-form $F_{J, H}(u) \in \Omega^{0,1}\left(\Sigma_{\Upsilon}^{\Lambda}, u^{*}\left(T T^{*} M\right)\right)$. 
A map $u$ in $C^{\infty}\left(\Sigma_{\Upsilon}^{\Omega}, T^{*} M\right)$ or in $C^{\infty}\left(\Sigma_{\Upsilon}^{\Lambda}, T^{*} M\right)$ solves equation (3-12) if and only if it solves the equation

$$
\bar{\partial}_{J, H}(u)=\partial_{s} u+J(u)\left(\partial_{t} u-X_{H}(t, u)\right)=0
$$

on $\operatorname{Int}\left(\Sigma_{\Upsilon}\right)$. If $u$ solves the above equation on $\left[s_{0}, s_{1}\right] \times\left[t_{0}, t_{1}\right]$, formula (3-6) together with an integration by parts leads to the identity

$$
\begin{aligned}
\int_{s_{0}}^{s_{1}} \int_{t_{0}}^{t_{1}}\left|\partial_{s} u(s, t)\right|^{2} d t d s & =\mathbb{A}_{H}^{\left[t_{0}, t_{1}\right]}\left(u\left(s_{0}, \cdot\right)\right)-\mathbb{A}_{H}^{\left[t_{0}, t_{1}\right]}\left(u\left(s_{1}, \cdot\right)\right) \\
& +\int_{s_{0}}^{s_{1}}\left(\eta\left(u\left(s, t_{1}\right)\right)\left[\partial_{s} u\left(s, t_{1}\right)\right]-\eta\left(u\left(s, t_{0}\right)\right)\left[\partial_{s} u\left(s, t_{0}\right)\right]\right) d s,
\end{aligned}
$$

where $\mathbb{A}_{H}^{I}(x)$ denotes the Hamiltonian action of the path $x$ on the interval $I$. We conclude that a solution $u$ of (3-12) on $\Sigma_{\Upsilon}^{\Lambda}$ or on $\Sigma_{\Upsilon}^{\Omega}$ - in the latter case with values in $T_{q_{0}}^{*} M$ on the boundary - satisfies the sharp energy identity

$$
\begin{aligned}
\int_{\Sigma_{\Upsilon} \cap\left\{|s| \leq s_{0}\right\}}\left|\partial_{s} u(s, t)\right|^{2} d s d t & \\
= & \mathbb{A}_{H}^{[-1,0]}\left(u\left(-s_{0}, \cdot\right)\right)+\mathbb{A}_{H}^{[0,1]}\left(u\left(-s_{0}, \cdot\right)\right)-\mathbb{A}_{H}^{[-1,1]}\left(u\left(s_{0}, \cdot\right)\right) .
\end{aligned}
$$

\subsection{The triangle and the pair-of-pants products}

Given $H_{1}, H_{2} \in C^{\infty}\left([0,1] \times T^{*} M\right)$ such that $H_{1}(1, \cdot)=H_{2}(0, \cdot)$ with all time derivatives, we define $H_{1} \# H_{2} \in C^{\infty}\left([0,1] \times T^{*} M\right)$ by

$$
H_{1} \# H_{2}(t, x)= \begin{cases}2 H_{1}(2 t, x) & \text { for } 0 \leq t \leq 1 / 2, \\ 2 H_{2}(2 t-1, x) & \text { for } 1 / 2 \leq t \leq 1\end{cases}
$$

Let us assume that $H_{1}, H_{2}$, and $H_{1} \# H_{2}$ satisfy $(\mathrm{H} 0)^{\Omega}$. The triangle product on $H F^{\Omega}\left(T^{*} M\right)$ will be induced by a chain map

$$
\Upsilon^{\Omega}: F_{h}^{\Omega}\left(H_{1}\right) \otimes F_{k}^{\Omega}\left(H_{2}\right) \rightarrow F_{h+k}^{\Omega}\left(H_{1} \# H_{2}\right) .
$$

In the periodic case, we consider Hamiltonians $H_{1}, H_{2} \in C^{\infty}\left(\mathbb{T} \times T^{*} M\right)$ such that $H_{1}(0, \cdot)=H_{2}(0, \cdot)$ with all time derivatives. Assuming that $H_{1}, H_{2}$, and $H_{1} \# H_{2}$ satisfy $(\mathrm{H} 0)^{\Lambda}$, the pair-of-pants product on $H F^{\Lambda}\left(T^{*} M\right)$ will be induced by a chain map

$$
\Upsilon^{\Lambda}: F_{h}^{\Lambda}\left(H_{1}\right) \otimes F_{k}^{\Lambda}\left(H_{2}\right) \rightarrow F_{h+k-n}^{\Lambda}\left(H_{1} \# H_{2}\right),
$$

where $n$ is the dimension of $M$. 
Let $H \in C^{\infty}\left([-1,1] \times T^{*} M\right)$, respectively $H \in C^{\infty}\left(\mathbb{R} / 2 \mathbb{Z} \times T^{*} M\right)$, be defined by

$$
H(t, x)=\frac{1}{2} H_{1} \# H_{2}((t+1) / 2, x)= \begin{cases}H_{1}(t+1, x) & \text { if }-1 \leq t \leq 0, \\ H_{2}(t, x) & \text { if } 0 \leq t \leq 1 .\end{cases}
$$

Notice that $x:[-1,1] \rightarrow T^{*} M$ is an orbit of $X_{H}$ if and only if the curve $t \mapsto$ $x((t+1) / 2)$ is an orbit of $X_{H_{1} \# H_{2}}$.

Given $x_{1} \in \mathscr{P}^{\Omega}\left(H_{1}\right), x_{2} \in \mathscr{P}^{\Omega}\left(H_{2}\right)$, and $y \in \mathscr{P}^{\Omega}\left(H_{1} \# H_{2}\right)$, consider the following space of solutions of the Floer equation $\bar{\partial}_{J, H}(u)=0$ on the holomorphic triangle:

$$
\begin{array}{r}
\mu_{\Upsilon}^{\Omega}\left(x_{1}, x_{2} ; y\right):=\left\{u \in C^{\infty}\left(\Sigma_{\Upsilon}^{\Omega}, T^{*} M\right) \mid \bar{\partial}_{J, H}(u)=0, u(z) \in T_{q_{0}}^{*} M \forall z \in \partial \Sigma_{\Upsilon}^{\Omega},\right. \\
\left.\lim _{s \rightarrow-\infty} u(s, t-1)=x_{1}(t), \lim _{s \rightarrow-\infty} u(s, t)=x_{2}(t), \lim _{s \rightarrow+\infty} u(s, 2 t-1)=y(t)\right\} .
\end{array}
$$

Similarly, for $x_{1} \in \mathscr{P}^{\Lambda}\left(H_{1}\right), x_{2} \in \mathscr{P} \Lambda\left(H_{2}\right)$, and $y \in \mathscr{P} \Lambda\left(H_{1} \# H_{2}\right)$, we consider the following space of solutions of the Floer equation on the pair-of-pants surface:

$$
\begin{array}{r}
\mu_{\Upsilon}^{\Lambda}\left(x_{1}, x_{2} ; y\right):=\left\{u \in C^{\infty}\left(\Sigma_{\Upsilon}^{\Lambda}, T^{*} M\right) \mid \bar{\partial}_{J, H}(u)=0,\right. \\
\lim _{s \rightarrow-\infty} u(s, t-1)=x_{1}(t), \\
\left.\lim _{s \rightarrow-\infty} u(s, t)=x_{2}(t), \lim _{s \rightarrow+\infty} u(s, 2 t-1)=y(t)\right\} .
\end{array}
$$

The following result is proved in Section 5.10.

Proposition 3.4 For a generic choice of $H_{1}$ and $H_{2}$ as above, the sets $\mu_{\Upsilon}^{\Omega}\left(x_{1}, x_{2} ; y\right)$ and $\mu_{\Upsilon}^{\Lambda}\left(x_{1}, x_{2} ; y\right)$ - if nonempty - are manifolds of dimension

$$
\begin{aligned}
& \operatorname{dim} \mu_{\Upsilon}^{\Omega}\left(x_{1}, x_{2} ; y\right)=\mu^{\Omega}\left(x_{1}\right)+\mu^{\Omega}\left(x_{2}\right)-\mu^{\Omega}(y), \\
& \operatorname{dim} \mu_{\Upsilon}^{\Lambda}\left(x_{1}, x_{2} ; y\right)=\mu^{\Lambda}\left(x_{1}\right)+\mu^{\Lambda}\left(x_{2}\right)-\mu^{\Lambda}(y)-n .
\end{aligned}
$$

These manifolds carry coherent orientations.

By the energy identity (3-15), every map $u$ in $\mu_{\Upsilon}^{\Omega}\left(x_{1}, x_{2} ; y\right)$ or in $\mu_{\Upsilon}^{\Lambda}\left(x_{1}, x_{2} ; y\right)$ satisfies

$$
\int_{\Sigma_{\Upsilon}}\left|\partial_{s} u(s, t)\right|^{2} d s d t=\mathbb{A}_{H_{1}}\left(x_{1}\right)+\mathbb{A}_{H_{2}}\left(x_{2}\right)-\mathbb{A}_{H_{1} \# H_{2}}(y) .
$$

As a consequence, we obtain the following compactness result, which is proved in Section 6.1.

Proposition 3.5 Assume that the Hamiltonians $H_{1}$ and $H_{2}$ satisfy (H1), (H2). Then the spaces $\mu_{\Upsilon}^{\Omega}\left(x_{1}, x_{2} ; y\right)$ and $\mu_{\Upsilon}^{\Lambda}\left(x_{1}, x_{2} ; y\right)$ are precompact in $C_{\mathrm{loc}}^{\infty}$. 
When $\mu^{\Omega}(y)=\mu^{\Omega}\left(x_{1}\right)+\mu^{\Omega}\left(x_{2}\right)$, then space $\mu_{\Upsilon}^{\Omega}\left(x_{1}, x_{2} ; y\right)$ is a finite set of oriented points, and we denote by $n_{\Upsilon}^{\Omega}\left(x_{1}, x_{2} ; y\right)$ the algebraic sum of the corresponding orientation signs. Similarly, when $\mu^{\Lambda}(y)=\mu^{\Lambda}\left(x_{1}\right)+\mu^{\Lambda}\left(x_{2}\right)-n$, the space $\mu_{\Upsilon}^{\Lambda}\left(x_{1}, x_{2} ; y\right)$ is a finite set of oriented points, and we denote by $n_{\Upsilon}^{\Lambda}\left(x_{1}, x_{2} ; y\right)$ the algebraic sum of the corresponding orientation signs. These integers are the coefficients of the homomorphisms

$$
\begin{aligned}
& \Upsilon^{\Omega}: F_{h}^{\Omega}\left(H_{1}\right) \otimes F_{k}^{\Omega}\left(H_{2}\right) \rightarrow F_{h+k}^{\Omega}\left(H_{1} \# H_{2}\right), \\
& x_{1} \otimes x_{2} \mapsto \sum_{\substack{y \in \mathscr{P}^{\Omega}\left(H_{1} \# H_{2}\right) \\
\mu^{\Omega}(y)=h+k}} n_{\Upsilon}^{\Omega}\left(x_{1}, x_{2} ; y\right) y, \\
& \Upsilon^{\Lambda}: F_{h}^{\Lambda}\left(H_{1}\right) \otimes F_{k}^{\Lambda}\left(H_{2}\right) \rightarrow F_{h+k-n}^{\Lambda}\left(H_{1} \# H_{2}\right), \\
& x_{1} \otimes x_{2} \mapsto \sum_{\substack{y \in \mathscr{P} \Lambda \\
\mu^{\Lambda}(y)=h+k-n}} n_{\Upsilon}^{\Lambda}\left(x_{1}, x_{2} ; y\right) y .
\end{aligned}
$$

A standard gluing argument shows that the homomorphisms $\Upsilon^{\Omega}$ and $\Upsilon^{\Lambda}$ are chain maps. Therefore, they define products

$$
\begin{aligned}
& H_{*} \Upsilon^{\Omega}: H F_{h}^{\Omega}\left(T^{*} M\right) \otimes H F_{k}^{\Omega}\left(T^{*} M\right) \rightarrow H F_{h+k}^{\Omega}\left(T^{*} M\right), \\
& H_{*} \Upsilon^{\Lambda}: H F_{h}^{\Lambda}\left(T^{*} M\right) \otimes H F_{k}^{\Lambda}\left(T^{*} M\right) \rightarrow H F_{h+k-n}^{\Lambda}\left(T^{*} M\right),
\end{aligned}
$$

in homology. Again by gluing arguments, it could be shown that these products have a unit element, are associative, and the second one is commutative. These facts will actually follow from the fact that these products correspond to the Pontrjagin and the loop products on $H_{*}\left(\Omega\left(M, q_{0}\right)\right)$ and $H_{*}(\Lambda(M))$.

\subsection{Factorization of the pair-of-pants product}

Let $H_{1}, H_{2} \in C^{\infty}\left(\mathbb{T} \times T^{*} M\right)$ be two Hamiltonians satisfying $(\mathrm{H} 0)^{\Lambda},(\mathrm{H} 1)$ and $(\mathrm{H} 2)$. We assume that $H_{1}(0, \cdot)=H_{2}(0, \cdot)$ with all time derivatives, so that the Hamiltonian $H_{1} \# H_{2}$ defined in (3-16) also belongs to $C^{\infty}\left(\mathbb{T} \times T^{*} M\right)$. We assume that $H_{1} \# H_{2}$ satisfies $(\mathrm{H} 0)^{\Lambda}$, while $H_{1} \oplus H_{2}$ satisfies $(\mathrm{H} 0)^{\Theta}$. The aim of this section is to construct two chain maps

$$
\begin{gathered}
E: F_{h}^{\Lambda}\left(H_{1}\right) \otimes F_{k}^{\Lambda}\left(H_{2}\right) \rightarrow F_{h+k-n}^{\Theta}\left(H_{1} \oplus H_{2}\right), \\
G: F_{k}^{\Theta}\left(H_{1} \oplus H_{2}\right) \rightarrow F_{k}^{\Lambda}\left(H_{1} \# H_{2}\right),
\end{gathered}
$$

such that the composition $G \circ E$ is chain homotopic to the pair-of-pants chain map $\Upsilon^{\Lambda}$. 
The homomorphisms $E$ is defined by counting solutions of the Floer equation on the Riemann surface $\Sigma_{E}$ which is the disjoint union of two closed disks with an inner and a boundary point removed. The homomorphism $G$ is defined by counting solutions of the Floer equation on the Riemann surface $\Sigma_{G}$ obtained by removing one inner point and two boundary points from the closed disk. Again, we find it useful to represent these Riemann surfaces as suitable quotients of strips with slits.

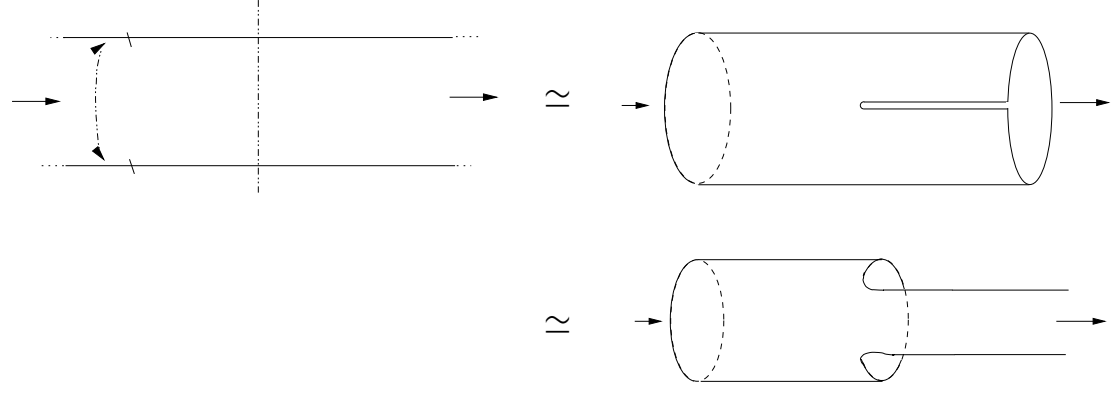

Figure 3: A component of $\Sigma_{E}$ : the cylinder with a slit

The surface $\Sigma_{E}$ can be described starting from the disjoint union of two strips,

$$
\mathbb{R} \times[-1,0] \sqcup \mathbb{R} \times[0,1],
$$

by making the following identifications:

$$
(s,-1) \sim\left(s, 0^{-}\right), \quad\left(s, 0^{+}\right) \sim(s, 1) \quad \text { for } s \leq 0 .
$$

The complex structure of $\Sigma_{E}$ is constructed by considering the holomorphic coordinate

$$
\{\zeta \in \mathbb{C}|\operatorname{Im} \zeta \geq 0,| \zeta \mid<1 / \sqrt{2}\} \rightarrow \Sigma_{E}, \quad \zeta \mapsto \begin{cases}\zeta^{2}-i & \text { if } \operatorname{Re} \zeta \geq 0, \\ \zeta^{2} & \text { if } \operatorname{Re} \zeta \leq 0,\end{cases}
$$

at $(0,-1) \sim\left(0,0^{-}\right)$, and the holomorphic coordinate

$$
\{\zeta \in \mathbb{C}|\operatorname{Im} \zeta \geq 0,| \zeta \mid<1 / \sqrt{2}\} \rightarrow \Sigma_{E}, \quad \zeta \mapsto \begin{cases}\zeta^{2}+i & \text { if } \operatorname{Re} \zeta \geq 0, \\ \zeta^{2} & \text { if } \operatorname{Re} \zeta \leq 0,\end{cases}
$$

at $\left(0,0^{+}\right) \sim(0,1)$. The resulting object is a Riemann surface consisting of two disjoint components, each of which is a cylinder with a slit: each component has one cylindrical end (on the left-hand side), one strip-like end and one boundary line (on the right-hand side). See Figure 3. The global holomorphic coordinate $z=s+i t$ has two singular points, at $\left(0,0^{-}\right) \sim(0,-1)$ and at $\left(0,0^{+}\right) \sim(0,1)$. 
The Riemann surface $\Sigma_{G}$ is obtained from the disjoint union of two strips $\mathbb{R} \times[-1,0] \sqcup$ $\mathbb{R} \times[0,1]$ by making the identifications

$$
\left\{\begin{array}{l}
\left(s, 0^{-}\right) \sim\left(s, 0^{+}\right) \\
(s,-1) \sim(s, 1)
\end{array} \quad \text { for } s \geq 0 .\right.
$$

A holomorphic coordinate at $(0,0)$ is the one given by (3-13), and a holomorphic coordinate at $(0,-1) \sim(0,1)$ is

$$
\{\zeta \in \mathbb{C}|\operatorname{Re} \zeta \geq 0,| \zeta \mid<1\} \rightarrow \Sigma_{G}, \quad \zeta \mapsto \begin{cases}\zeta^{2}-i & \text { if } \operatorname{Im} \zeta \geq 0, \\ \zeta^{2}+i & \text { if } \operatorname{Im} \zeta \leq 0,\end{cases}
$$

We obtain a Riemann surface with two boundary lines and two strip-like ends (on the left-hand side), and a cylindrical end (on the right-hand side). The global holomorphic coordinate $z=s+i t$ has two singular points, at $(0,0)$, and at $(0,-1) \sim(0,1)$.

Let $H \in C^{\infty}\left(\mathbb{R} / 2 \mathbb{Z} \times T^{*} M\right)$ be defined by (3-17). Given $x_{1} \in \mathscr{P} \Lambda\left(H_{1}\right), x_{2} \in \mathscr{P} \Lambda\left(H_{2}\right)$, $y=\left(y_{1}, y_{2}\right) \in \mathscr{P}^{\Theta}\left(H_{1} \oplus H_{2}\right)$, and $z \in \mathscr{P}^{\Lambda}\left(H_{1} \# H_{2}\right)$, we consider the following spaces of maps. The set $\mu_{E}\left(x_{1}, x_{2} ; y\right)$ is the space of solutions $u \in C^{\infty}\left(\Sigma_{E}, T^{*} M\right)$ of the Floer equation

$$
\bar{\partial}_{J, H}(u)=0,
$$

satisfying the boundary conditions

$$
\left\{\begin{array}{l}
\pi \circ u(s,-1)=\pi \circ u\left(s, 0^{-}\right)=\pi \circ u\left(s, 0^{+}\right)=\pi \circ u(s, 1), \\
u\left(s, 0^{-}\right)-u(s,-1)+u(s, 1)-u\left(s, 0^{+}\right)=0,
\end{array} \quad \forall s \geq 0,\right.
$$

and the asymptotic conditions

$$
\begin{aligned}
& \lim _{s \rightarrow-\infty} u(s, t-1)=x_{1}(t), \quad \lim _{s \rightarrow-\infty} u(s, t)=x_{2}(t), \\
& \lim _{s \rightarrow+\infty} u(s, t-1)=y_{1}(t), \quad \lim _{s \rightarrow+\infty} u(s, t)=y_{2}(t) .
\end{aligned}
$$

The set $M_{G}(y, z)$ is the set of solutions $u \in C^{\infty}\left(\Sigma_{G}, T^{*} M\right)$ of the same equation, the same boundary conditions but for $s \leq 0$, and the asymptotic conditions

$$
\lim _{s \rightarrow-\infty} u(s, t-1)=y_{1}(t), \quad \lim _{s \rightarrow-\infty} u(s, t)=y_{2}(t), \quad \lim _{s \rightarrow+\infty} u(s, 2 t-1)=z(t) .
$$

The following result is proved in Section 5.10.

Proposition 3.6 For a generic choice of $H_{1}$ and $H_{2}$, the spaces $\mu_{E}\left(x_{1}, x_{2} ; y\right)$ and $\mu_{G}(y, z)$ - if nonempty - are manifolds of dimension

$$
\begin{aligned}
\operatorname{dim} \mu_{E}\left(x_{1}, x_{2} ; y\right) & =\mu^{\Lambda}\left(x_{1}\right)+\mu^{\Lambda}\left(x_{2}\right)-\mu^{\Theta}(y)-n, \\
\operatorname{dim} \mu_{G}(y, z) & =\mu^{\Theta}(y)-\mu^{\Lambda}(z) .
\end{aligned}
$$

These manifolds carry coherent orientations. 
The energy identities are now

$$
\int_{\Sigma_{E}}\left|\partial_{s} u(s, t)\right|^{2} d s d t=\mathbb{A}_{H_{1}}\left(x_{1}\right)+\mathbb{A}_{H_{2}}\left(x_{2}\right)-\mathbb{A}_{H_{1} \oplus H_{2}}(y),
$$

for every $u \in \mathcal{M}_{E}\left(x_{1}, x_{2} ; y\right)$, and

$$
\int_{\Sigma_{G}}\left|\partial_{s} u(s, t)\right|^{2} d s d t=\mathbb{A}_{H_{1} \oplus H_{2}}(y)-\mathbb{A}_{H_{1} \# H_{2}}(z),
$$

for every $u \in M_{G}(y, z)$. As usual, they imply the following compactness result (proved in Section 6.1).

Proposition 3.7 Assume that $H_{1}$ and $H_{2}$ satisfy $(\mathrm{H} 1)$ and (H2). Then the spaces $\mu_{E}\left(x_{1}, x_{2} ; y\right)$ and $\mu_{G}(y, z)$ are precompact in $C_{\text {loc }}^{\infty}$.

When $\mu^{\Theta}(y)=\mu^{\Lambda}\left(x_{1}\right)+\mu^{\Lambda}\left(x_{2}\right)-n, \mu_{E}\left(x_{1}, x_{2} ; y\right)$ is a finite set of oriented points, and we denote by $n_{E}\left(x_{1}, x_{2} ; y\right)$ the algebraic sum of the corresponding orientation signs. Similarly, when $\mu^{\Lambda}(z)=\mu^{\Theta}(y), \mu_{G}(y, z)$ is a finite set of oriented points, and we denote by $n_{G}(y, z)$ the algebraic sum of the corresponding orientation signs. These integers are the coefficients of the homomorphisms

$$
\begin{aligned}
E: F_{h}^{\Lambda}\left(H_{1}\right) \otimes F_{k}^{\Lambda}\left(H_{2}\right) & \rightarrow F_{h+k-n}^{\Theta}\left(H_{1} \oplus H_{2}\right), \\
x_{1} \otimes x_{2} & \mapsto \sum_{\substack{y \in \mathscr{P} \\
\mu^{\Theta}(y)=h+k-n}} n_{E}\left(x_{1}, x_{2} ; y\right) y, \\
G: F_{k}^{\Theta}\left(H_{1} \oplus H_{2}\right) & \rightarrow F_{k}^{\Lambda}\left(H_{1} \# H_{2}\right), \\
y & \sum_{\substack{z \in \mathscr{P}^{\Lambda}\left(H_{1} \# H_{2}\right) \\
\mu^{\Lambda}(z)=k}} n_{G}(y, z) z .
\end{aligned}
$$

A standard gluing argument shows that these homomorphisms are chain maps. The main result of this section states that the pair-of-pants product on $T^{*} M$ factors through the Floer homology of figure-8 loops. More precisely, the following chain level result holds:

Theorem 3.8 The chain maps

$$
\Upsilon^{\Lambda}, G \circ E:\left(F^{\Lambda}\left(H_{1}\right) \otimes F^{\Lambda}\left(H_{2}\right)\right)_{k}=\bigoplus_{j+h=k} F_{j}^{\Lambda}\left(H_{1}\right) \otimes F_{h}^{\Lambda}\left(H_{2}\right) \longrightarrow F_{k-n}^{\Lambda}\left(H_{1} \# H_{2}\right)
$$

are chain homotopic.

In order to prove the above theorem, we must construct a homomorphism

$$
P_{G E}^{\Upsilon}:\left(F^{\Lambda}\left(H_{1}\right) \otimes F^{\Lambda}\left(H_{2}\right)\right)_{k} \rightarrow F_{k-n+1}^{\Lambda}\left(H_{1} \# H_{2}\right),
$$


such that

$$
\begin{aligned}
& \left(\Upsilon^{\Lambda}-G \circ E\right)(\alpha \otimes \beta) \\
& \quad=\partial_{H_{1} \# H_{2}}^{\Lambda} \circ P_{G E}^{\Upsilon}(\alpha \otimes \beta)+P_{G E}^{\Upsilon}\left(\partial_{H_{1}}^{\Lambda} \alpha \otimes \beta+(-1)^{h} \alpha \otimes \partial_{H_{2}}^{\Lambda} \beta\right),
\end{aligned}
$$

for every $\alpha \in F_{h}^{\Lambda}\left(H_{1}\right)$ and $\beta \in F_{j}^{\Lambda}\left(H_{2}\right)$. The chain homotopy $P_{G E}^{\Upsilon}$ is defined by counting solutions of the Floer equation on a one-parameter family of Riemann surfaces with boundary $\left.\Sigma_{G E}^{\Upsilon}(\alpha), \alpha \in\right] 0,+\infty[$, obtained by removing an open disks from the pair-of-pants.

More precisely, given $\alpha \in] 0,+\infty\left[\right.$, we define $\Sigma_{G E}^{\Upsilon}(\alpha)$ as the quotient of the disjoint union $\mathbb{R} \times[-1,0] \sqcup \mathbb{R} \times[0,1]$ under the identifications

$$
\left\{\begin{array}{l}
(s,-1) \sim\left(s, 0^{-}\right) \\
\left(s, 0^{+}\right) \sim(s, 1)
\end{array} \quad \text { if } s \leq 0, \quad\left\{\begin{array}{l}
(s,-1) \sim(s, 1) \\
\left(s, 0^{-}\right) \sim\left(s, 0^{+}\right)
\end{array} \quad \text { if } s \geq \alpha .\right.\right.
$$

This object is a Riemann surface with boundary, with the holomorphic coordinates (3-19) and (3-20) at $(0,-1) \sim\left(0,0^{-}\right)$and at $\left(0,0^{+}\right) \sim(0,1)$, and with the holomorphic coordinates (3-13) and (3-21) (translated by $\alpha)$ at $(\alpha, 0)$ and at $(\alpha,-1) \sim(\alpha, 1)$. The resulting object is a Riemann surface with three cylindrical ends and one boundary circle. Given $x_{1} \in \mathscr{P} \Lambda\left(H_{1}\right), x_{2} \in \mathscr{P} \Lambda\left(H_{2}\right)$ and $z \in \mathscr{P} \Lambda\left(H_{1} \# H_{2}\right)$, we define $\mu_{G E}^{\Upsilon}\left(x_{1}, x_{2} ; z\right)$ to be the space of pairs $(\alpha, u)$, with $\alpha>0$ and $u \in C^{\infty}\left(\Sigma_{G E}^{\Upsilon}(\alpha), T^{*} M\right)$ the solution of

$$
\bar{\partial}_{J, H}(u)=0,
$$

with boundary conditions

$$
\left\{\begin{array}{l}
\pi \circ u(s,-1)=\pi \circ u\left(s, 0^{-}\right)=\pi \circ u\left(s, 0^{+}\right)=\pi \circ u(s, 1), \\
u\left(s, 0^{-}\right)-u(s,-1)+u(s, 1)-u\left(s, 0^{+}\right)=0,
\end{array} \quad \forall s \in[0, \alpha],\right.
$$

and asymptotic conditions

$$
\lim _{s \rightarrow-\infty} u(s, t-1)=x_{1}(t), \quad \lim _{s \rightarrow-\infty} u(s, t)=x_{2}(t), \quad \lim _{s \rightarrow+\infty} u(s, 2 t-1)=z(t) .
$$

The following result is proved in Section 5.10.

Proposition 3.9 For a generic choice of $H_{1}$ and $H_{2}, \mu_{G E}^{\Upsilon}\left(x_{1}, x_{2} ; z\right)$ - if nonempty - is a manifold of dimension

$$
\operatorname{dim} \mu_{G E}^{\Upsilon}\left(x_{1}, x_{2} ; z\right)=\mu^{\Lambda}\left(x_{1}\right)+\mu^{\Lambda}\left(x_{2}\right)-\mu^{\Lambda}(z)-n+1 .
$$

The projection $(\alpha, u) \mapsto \alpha$ is smooth on $\mathcal{M}_{G E}^{\Upsilon}\left(x_{1}, x_{2} ; z\right)$. These manifolds carry coherent orientations. 
Energy estimates, together with (H1) and (H2), again imply compactness. When $\mu^{\Lambda}(z)=\mu^{\Lambda}\left(x_{1}\right)+\mu^{\Lambda}\left(x_{2}\right)-n+1, \mu_{G E}^{\Upsilon}\left(x_{1}, x_{2} ; z\right)$ is a finite set of oriented points. Denoting by $n_{G E}^{\Upsilon}\left(x_{1}, x_{2} ; z\right)$ the algebraic sum of the corresponding orientation signs, we define the homomorphism

$$
\begin{aligned}
P_{G E}^{\Upsilon}: F_{h}^{\Lambda}\left(H_{1}\right) \otimes F_{k}^{\Lambda}\left(H_{2}\right) & \rightarrow F_{h+k-n+1}^{\Lambda}\left(H_{1} \# H_{2}\right), \\
x_{1} \otimes x_{2} & \mapsto \sum_{\substack{z \in \mathscr{P} \Lambda \\
\mu^{\Lambda}(z)=h+k-n+1}}^{\Upsilon} n_{G E}^{\Upsilon}\left(x_{1}, x_{2} ; z\right) z .
\end{aligned}
$$

Then Theorem 3.8 is a consequence of the following:

Proposition 3.10 The homomorphism $P_{G E}^{\Upsilon}$ is a chain homotopy between $\Upsilon^{\Lambda}$ and $G \circ E$.

The proof of the above result is contained in Section 6.3.

\subsection{The homomorphisms C, Ev and I!}

In this section we define the Floer homological counterparts of the homomorphisms

$$
\begin{gathered}
\mathrm{c}_{*}: H_{k}(M) \rightarrow H_{k}(\Lambda(M)), \quad \mathrm{ev}_{*}: H_{k}(\Lambda(M)) \rightarrow H_{k}(M), \\
\mathrm{i}_{!}: H_{k}(\Lambda(M)) \rightarrow H_{k-n}\left(\Omega\left(M, q_{0}\right)\right) .
\end{gathered}
$$

These are the homomorphism which will appear in the Floer homological counterpart of diagram (1-1). The reader who is interested only in Theorems A and B of the Introduction may skip this section.

The homomorphisms $\mathrm{C}$ and Ev Let $f$ be a smooth Morse function on $M$, and assume that the vector field $-\operatorname{grad} f$ satisfies the Morse-Smale condition. Let $H \in$ $C^{\infty}\left(\mathbb{T} \times T^{*} M\right)$ be a Hamiltonian which satisfies $(\mathrm{H} 0)^{\Lambda},(\mathrm{H} 1),(\mathrm{H} 2)$. We shall define two chain maps

$$
\mathrm{C}: M_{k}(f) \rightarrow F_{k}(H), \quad \text { Ev: } F_{k}(H) \rightarrow M_{k}(f) .
$$

Given $x \in \operatorname{crit}(f)$ and $y \in \mathscr{P}^{\Lambda}(H)$, consider the following spaces of maps

$$
\begin{array}{r}
\mu_{\mathrm{C}}(x, y)=\left\{u \in C ^ { \infty } \left(\left[0,+\infty\left[\times \mathbb{T}, T^{*} M\right) \mid \bar{\partial}_{J, H}(u)=0,\right.\right.\right. \\
\left.\pi \circ u(0, t) \equiv q \in W^{u}(x) \forall t \in \mathbb{T}, \lim _{s \rightarrow+\infty} u(s, t)=y(t)\right\}, \\
\left.\mu_{\mathrm{Ev}}(y, x)=\left\{u \in C^{\infty}(]-\infty, 0\right] \times \mathbb{T}, T^{*} M\right) \mid \bar{\partial}_{J, H}(u)=0, u(0, t) \in \mathbb{O}_{M} \forall t \in \mathbb{T}, \\
\left.u(0,0) \in W^{s}(x), \lim _{s \rightarrow-\infty} u(s, t)=y(t)\right\},
\end{array}
$$


where $\mathbb{O}_{M}$ denotes the image of the zero section in $T^{*} M$. The following result is proved in Section 5.10.

Proposition 3.11 For a generic choice of $H$ and $f, \mu_{C}(x, y)$ and $\mu_{\mathrm{Ev}}(y, x)$ are manifolds with

$$
\operatorname{dim} \mu_{\mathrm{C}}(x, y)=i(x)-\mu^{\Lambda}(y), \quad \operatorname{dim} \mu_{\mathrm{Ev}}(y, x)=\mu^{\Lambda}(y)-i(x) .
$$

These manifolds carry coherent orientations.

If $u$ belongs to $\mu_{\mathrm{C}}(x, y)$ or $\mu_{\mathrm{Ev}}(y, x)$, the fact that $u(0, \cdot)$ takes value either on the fiber of some point $q \in M$ or on the zero section of $T^{*} M$ implies that

$$
\mathbb{A}_{H}(u(0, \cdot))=-\int_{0}^{1} H(t, u(0, t)) d t .
$$

Therefore, we have the energy estimates

$$
\int_{[0,+\infty[\times \mathbb{T}}\left|\partial_{s} u(s, t)\right|^{2} d s d t \leq-\min H-\mathbb{A}_{H}(y),
$$

for every $u \in M_{\mathrm{C}}(x, y)$, and

$$
\int_{]-\infty, 0] \times \mathbb{T}}\left|\partial_{s} u(s, t)\right|^{2} d s d t \leq \mathbb{A}_{H}(y)+\max _{(t, q) \in \mathbb{T} \times M} H(t, q, 0),
$$

for every $u \in M_{\mathrm{Ev}}(y, x)$. These energy estimates allow to prove the following compactness result:

Proposition 3.12 The spaces $\mu_{\mathrm{C}}(x, y)$ and $\mu_{\mathrm{Ev}}(y, x)$ are precompact in

$$
C_{\mathrm{loc}}^{\infty}\left(\left[0,+\infty\left[\times \mathbb{T}, T^{*} M\right) \text { and } C_{\mathrm{loc}}^{\infty}(]-\infty, 0\right] \times \mathbb{T}, T^{*} M\right) .
$$

When $\mu^{\Lambda}(y)=i(x), \mu_{\mathrm{C}}(x, y)$ and $\mu_{\mathrm{Ev}}(y, x)$ consist of finitely many oriented points. The algebraic sums of these orientation signs, denoted by $n_{\mathrm{C}}(x, y)$ and $n_{\mathrm{Ev}}(y, x)$, define the homomorphisms

$$
\begin{aligned}
& \mathrm{C}: M_{k}(f) \rightarrow F_{k}^{\Lambda}(H), \quad x \mapsto \sum_{\substack{y \in \mathscr{P} \Lambda \\
\mu^{\Lambda}(y)=k}} n_{\mathrm{C}}(x, y) y, \\
& \text { Ev: } F_{k}^{\Lambda}(H) \rightarrow M_{k}(f), \quad y \mapsto \sum_{\substack{x \in \operatorname{crit}(f) \\
i(x)=k}} n_{\mathrm{Ev}}(y, x) x .
\end{aligned}
$$

A standard gluing argument shows that $\mathrm{C}$ and $\mathrm{Ev}$ are chain maps. The induced homomorphisms in homology are denoted by $\mathrm{C}_{*}$ and $\mathrm{Ev}_{*}$. 
The homomorphism I! Let $\Sigma_{I_{!}}$be a cylinder with a slit. More precisely, $\Sigma_{I_{!}}$is obtained from the strip $\mathbb{R} \times[0,1]$ by the identifications $(s, 0) \sim(s, 1)$ for every $s \leq 0$. At the point $(0,0) \sim(0,1)$ we have the holomorphic coordinate

$$
\{\zeta \in \mathbb{C}|\operatorname{Re} \zeta \geq 0,| \zeta \mid<1 / \sqrt{2}\} \rightarrow \Sigma_{I_{!}}, \quad \zeta \mapsto \begin{cases}\zeta^{2} & \text { if } \operatorname{Im} \zeta \geq 0, \\ \zeta^{2}+i & \text { if } \operatorname{Im} \zeta \leq 0 .\end{cases}
$$

It is a Riemann surface with one cylindrical end (on the left-hand side), one strip-like end, and one boundary line (on the right-hand side). It is the copy of one component of $\Sigma_{E}$; see Figure 3 .

Consider now a Hamiltonian $H \in C^{\infty}\left(\mathbb{T} \times T^{*} M\right)$ which satisfies $(\mathrm{H} 0)^{\Lambda},(\mathrm{H} 0)^{\Omega}$, (H1), (H2). We also assume the condition

$$
x \in \mathscr{P}^{\Lambda}(H) \quad \Longrightarrow \quad x(0) \notin T_{q_{0}}^{*} M .
$$

Given $x \in \mathscr{P}^{\Lambda}(H)$ and $y \in \mathscr{P}^{\Omega}(H)$, we introduce the space of maps

$$
\begin{aligned}
M_{I_{!}}(x, y)= & \left\{u \in C^{\infty}\left(\Sigma_{I_{!}}, T^{*} M\right) \mid \bar{\partial}_{J, H}(u)=0, u(s, 0) \in T_{q_{0}}^{*} M\right. \text { and } \\
& \left.u(s, 1) \in T_{q_{0}}^{*} M \forall s \geq 0, \lim _{s \rightarrow-\infty} u(s, t)=x(t), \lim _{s \rightarrow+\infty} u(s, t)=y(t)\right\} .
\end{aligned}
$$

The following result is proved in Section 5.10:

Proposition 3.13 For a generic $H$ satisfying (3-25), the space $M_{I !}(x, y)$ is a manifold with

$$
\operatorname{dim} \mu_{I !}(x, y)=\mu^{\Lambda}(x)-\mu^{\Omega}(y)-n .
$$

These manifolds carry coherent orientations.

The general discussion of Section 6.1 gives the following compactness statement:

Proposition 3.14 The space $\mu_{I_{!}}(x, y)$ is precompact in $C_{\mathrm{loc}}^{\infty}\left(\Sigma_{I_{!}}, T^{*} M\right)$.

When $\mu^{\Omega}(y)=\mu^{\Lambda}(x)-n$, the space $\mu_{I !}(x, y)$ consists of finitely many oriented points. The algebraic sum of these orientations is denoted by $n_{I !}(x, y)$, and defines the homomorphism

$$
I_{!}: F_{k}^{\Lambda}(H) \rightarrow F_{k-n}^{\Omega}(H), \quad x \mapsto \sum_{\substack{y \in \mathscr{P}^{\Omega} \\ \mu^{\Omega}(y)=k-n}} n_{I_{!}}(x, y) y .
$$

A standard gluing argument shows that $I_{!}$is a chain map. The induced map in homology is denoted by the same symbol. 


\section{Isomorphisms between Morse and Floer complexes}

\subsection{The chain complex isomorphism}

Let $Q$ be a closed manifold and let $R$ be a closed submanifold of $Q \times Q$, as in Section 2.1 and Section 3.1. The aim of this section is to recall the construction of an isomorphism between $H F_{*}^{R}\left(T^{*} Q\right)$, the Floer homology of $T^{*} Q$ with nonlocal conormal boundary conditions defined by $R$ (see Section 3.1), and the singular homology of the path space

$$
P_{R}(Q):=\left\{\gamma \in C^{0}([0,1], Q) \mid(\gamma(0), \gamma(1)) \in R\right\} .
$$

The existence of such an isomorphism was first proved by Viterbo in [52], in the case of periodic boundary conditions (that is, when $R$ is the diagonal in $Q \times Q$ ). A different proof is due to Salamon and Weber [44]. Here we adopt a third approach, which we have introduced in [4] for periodic and Dirichlet boundary conditions, and later extended to arbitrary nonlocal conormal boundary conditions with Portaluri in [2]. See also Weber [54] for a nice exposition comparing the three approaches.

The strategy is to choose the Hamiltonian $H \in C^{\infty}\left([0,1] \times T^{*} Q\right)$ to be the Fenchel dual of a Lagrangian $L \in C^{\infty}([0,1] \times T Q)$, and to work at the chain level, by constructing a chain isomorphism

$$
\Phi_{L}^{R}: M_{*}\left(\mathbb{S}_{L}^{R}\right) \rightarrow F_{*}^{R}(H)
$$

from the Morse complex of the Lagrangian action functional $\mathbb{S}_{L}^{R}$ introduced in Section 2.1 to the Floer complex of $H$. More precisely, we assume that the Lagrangian $L$ satisfies the assumptions (L1) and (L2) and that all the solutions $\gamma$ in $\mathscr{P}^{R}(L)$ are nondegenerate. It follows that the Fenchel dual Hamiltonian $H$, which is defined by

$$
H(t, q, p):=\max _{v \in T_{q} M}(\langle p, v\rangle-L(t, q, v)),
$$

is smooth and satisfies (H0), (H1) and (H2). If $v(t, q, p) \in T_{q} M$ is the (unique) vector where the above maximum is achieved, the map

$$
[0,1] \times T^{*} M \rightarrow[0,1] \times T M, \quad(t, q, p) \mapsto(t, q, v(t, q, p)),
$$

is a diffeomorphism, called the Legendre transform associated to the Lagrangian $L$. There is a one-to-one correspondence $x \mapsto \pi \circ x$ between the orbits of the Hamiltonian vector field $X_{H}$ and the solutions of the Euler-Lagrange equation (2-1) associated to $L$, such that $\left(t, \pi \circ x(t),(\pi \circ x)^{\prime}(t)\right)$ is the Legendre transform of $(t, x(t))$. Therefore, $x$ belongs to $\mathscr{P}^{R}(H)$ if and only if $\pi \circ x$ belongs to $\mathscr{P}^{R}(L)$, and the fact that $\pi \circ x$ is nondegenerate is equivalent to the fact that $x$ is nondegenerate. Therefore, both the Morse complex $M_{*}\left(\mathbb{S}_{L}^{R}\right)$ and the Floer complex $F_{*}^{R}(H)$ are well-defined. The 
existence of the isomorphism (4-1) implies that the Floer homology $H F_{*}^{R}\left(T^{*} Q\right)$ is isomorphic to the singular homology of $P_{Q}(R)$, just because the Morse homology $H M_{*}\left(\mathbb{S}_{L}^{R}\right)$ of the functional $\mathbb{S}_{L}^{R}$ is isomorphic to the singular homology of its domain $W_{R}^{1,2}([0,1], Q)$, and because the latter space is homotopically equivalent to $P_{Q}(R)$.

Clearly, the identification between generators $\mathscr{P}^{R}(H) \rightarrow \mathscr{P}^{R}(L), x \mapsto \pi \circ x$, need not produce a chain map, because the definitions of the boundary operator in the two complexes have little in common. The construction of the isomorphism $\Phi_{L}^{R}$ is based instead on counting solutions of a hybrid problem, that we now describe.

Given $\gamma \in \mathscr{P}^{R}(L)$ and $x \in \mathscr{P}^{R}(H)$, we denote by $\mu_{\Phi}^{R}(\gamma, x)$ the space of maps $u:\left[0,+\infty\left[\times[0,1] \rightarrow T^{*} Q\right.\right.$ which solve the Floer equation

$$
\bar{\partial}_{J, H}(u)=0,
$$

together with the asymptotic condition

$$
\lim _{s \rightarrow+\infty} u(s, t)=x(t)
$$

and the boundary conditions

$$
(u(s, 0), \mathscr{b} u(s, 1)) \in N^{*} R, \quad \forall s \geq 0, \quad \pi \circ u(0, \cdot) \in W^{u}(\gamma),
$$

where $W^{u}(\gamma) \subset W_{R}^{1,2}([0,1], Q)$ denotes the unstable manifold of $\gamma$ with respect to the pseudo-gradient vector field $X$ for $\mathbb{S}_{L}^{R}$ used in the definition of the Morse complex of $\mathbb{S}_{L}^{R}$. For a generic choice of $L$ and of the pseudo-gradient $X$, these spaces of maps are manifolds of dimension

$$
\operatorname{dim} \mu_{\Phi}^{R}(\gamma, x)=i\left(\gamma ; \mathbb{S}_{L}^{R}\right)-\mu^{R}(x),
$$

where $i\left(\gamma ; \mathbb{S}_{L}^{R}\right)$ denotes the Morse index of $\gamma$, seen as a critical point of $\mathbb{S}_{L}^{R}$, and $\mu^{R}(x)$ is the Maslov index of $x$ defined in (3-5). The fact that $H$ is the Fenchel dual of $L$ immediately implies the following important inequality between the Hamiltonian and the Lagrangian action functionals:

$$
\mathbb{A}_{H}(x) \leq \mathbb{S}_{L}(\pi \circ x), \quad \forall x \in C^{\infty}\left([0,1], T^{*} Q\right),
$$

with the equality holding if and only if $x$ is related to $\left(\pi \circ x,(\pi \circ x)^{\prime}\right)$ by the Legendre transform. In particular, the equality holds if $x$ is an orbit of the Hamiltonian vector field $X_{H}$. The inequality (4-4) provides us with the energy estimates which allow to prove suitable compactness properties for the spaces $\mu_{\Phi}^{R}(\gamma, x)$. When $\mu^{R}(x)=$ $i\left(\gamma ; \mathbb{S}_{L}^{R}\right)$, the space $M_{\Phi}^{R}(\gamma, x)$ consists of finitely many oriented points, which add up 
to the integers $n_{\Phi}^{R}(\gamma, x)$. These integers are the coefficients of the homomorphism

$$
\Phi_{L}^{R}: M_{k}\left(\mathbb{S}_{L}^{R}\right) \rightarrow F_{k}^{R}(H, J), \quad \gamma \mapsto \sum_{\substack{x \in \mathscr{P}^{R}(H) \\ \mu^{R}(x)=k}} n_{\Phi}^{R}(\gamma, x) x,
$$

which can be shown to be a chain map. The inequality (4-4) implies that $n_{\Phi}^{R}(\gamma, x)=0$ if $\mathbb{A}_{H}(x) \geq \mathbb{S}_{L}(\gamma)$ and $\gamma \neq \pi \circ x$, while $n_{\Phi}^{R}(\gamma, x)= \pm 1$ if $\gamma=\pi \circ x$. These facts imply that $\Phi_{L}^{R}$ is an isomorphism.

As in Section 2.1 and Section 3.1, we are interested in the boundary conditions given by the following choices for $R$ : Periodic ( $Q=M$ and $R=\Delta_{M}$ ), Dirichlet ( $Q=M$ and $\left.R=\left(q_{0}, q_{0}\right)\right)$, Figure-8 $\left(Q=M \times M, R=\Delta_{M}^{(4)}\right)$. We denote the corresponding isomorphisms also by the symbols

$$
\Phi_{L}^{\Lambda}:=\Phi_{L}^{\Delta_{M}}, \quad \Phi_{L}^{\Omega}:=\Phi_{L}^{\left(q_{0}, q_{0}\right)}, \quad \Phi_{L}^{\Theta}:=\Phi_{L}^{\Delta_{M}^{(4)}} .
$$

\subsection{A chain level proof of Theorem B}

Theorem B of the Introduction says that if $M$ is a closed manifold and $q_{0} \in M$, then there is a graded ring isomorphism

$$
H_{*}\left(\Omega\left(M, q_{0}\right)\right) \cong H F_{*}^{\Omega}\left(T^{*} M\right)
$$

where the first graded group is endowed with the Pontrjagin product \# (see Section 1.1), and the second one with the triangle product $\Upsilon_{*}^{\Omega}$ (see Section 3.3). By the results of Section 2.3, the Pontrjagin product \# can be read on the Morse complex of the Lagrangian action functional with Dirichlet boundary conditions as the chain map

$$
M_{\#}: M_{j}\left(\mathbb{S}_{L_{1}}^{\Omega}\right) \otimes M_{k}\left(\mathbb{S}_{L_{2}}^{\Omega}\right) \rightarrow M_{j+k}\left(\mathbb{S}_{L_{1} \# L_{2}}^{\Omega}\right),
$$

defined in Proposition 2.2. Therefore, Theorem B is implied by the following chain level statement, which is the main result of this section:

Theorem 4.1 Let $L_{1}, L_{2} \in C^{\infty}([0,1] \times T M)$ be two Lagrangians which satisfy $(\mathrm{L} 0)^{\Omega},(\mathrm{L} 1),(\mathrm{L} 2)$, are such that $L_{1}(1, \cdot)=L_{2}(0, \cdot)$ with all the time derivatives, and (2-9) holds. Assume also that the Lagrangian $L_{1} \# L_{2}$ defined by (2-8) satisfies (L0) ${ }^{\Omega}$. Let $H_{1}$ and $H_{2}$ be the Fenchel transforms of $L_{1}$ and $L_{2}$, so that $H_{1} \# H_{2}$ is the Fenchel transform of $L_{1} \# L_{2}$, and the three Hamiltonians $H_{1}, H_{2}$, and $H_{1} \# H_{2}$ satisfy $(\mathrm{H} 0)^{\Omega}$, 
(H1), (H2). Then the diagram

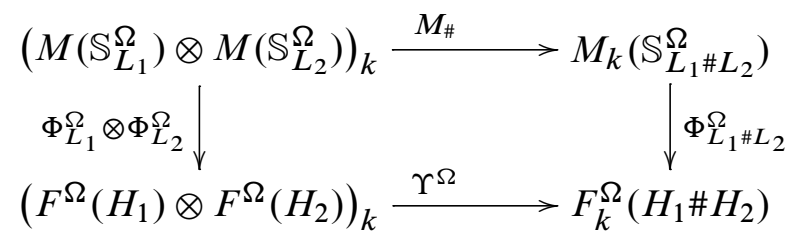

is chain-homotopy commutative.

Instead of constructing directly a homotopy between $\Phi_{L_{1} \# L_{2}}^{\Omega} \circ M_{\#}$ and $\Upsilon^{\Omega} \circ \Phi_{L_{1}}^{\Omega} \otimes \Phi_{L_{2}}^{\Omega}$, we shall prove that both these chain maps are homotopic to a third one, that we name $K^{\Omega}$; see Figure 4.
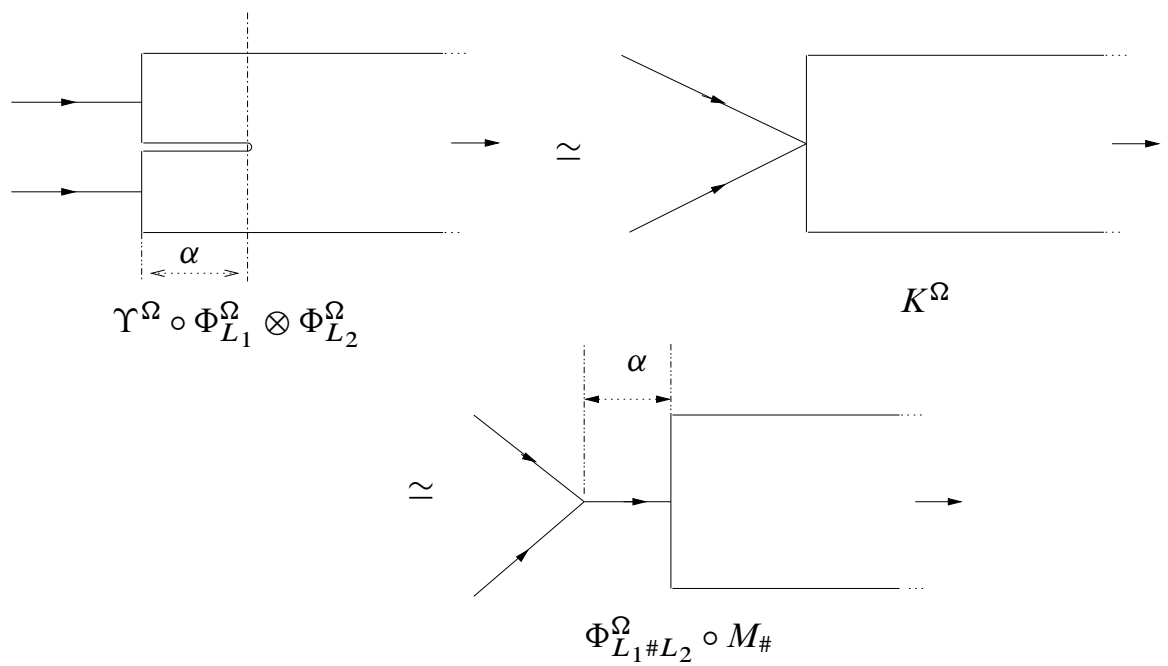

Figure 4: The homotopy through $K^{\Omega}$

The definition of $K^{\Omega}$ is based on the following space of solutions of the Floer equation for the Hamiltonian $H$ defined in (3-17): given $\gamma_{1} \in \mathscr{P}^{\Omega}\left(L_{1}\right), \gamma_{2} \in \mathscr{P}^{\Omega}\left(L_{2}\right)$, and $x \in \mathscr{P}^{\Omega}\left(H_{1} \# H_{2}\right)$, let $\mathcal{M}_{K}^{\Omega}\left(\gamma_{1}, \gamma_{2} ; x\right)$ be the space of solutions $u:[0,+\infty[\times[-1,1] \rightarrow$ $T^{*} M$ of the Floer equation

$$
\bar{\partial}_{J, H}(u)=0,
$$

with boundary conditions

$$
\begin{gathered}
\pi \circ u(s,-1)=\pi \circ u(s, 1)=q_{0}, \quad \forall s \geq 0, \\
\pi \circ u(0, \cdot-1) \in W^{u}\left(\gamma_{1} ; X_{L_{1}}^{\Omega}\right), \quad \pi \circ u(0, \cdot) \in W^{u}\left(\gamma_{2} ; X_{L_{2}}^{\Omega}\right),
\end{gathered}
$$


and the asymptotic behavior

$$
\lim _{s \rightarrow+\infty} u(s, 2 t-1)=x(t) .
$$

Here, $X_{L_{1}}^{\Omega}$ and $X_{L_{2}}^{\Omega}$ are Morse-Smale pseudo-gradients for the functionals $\mathbb{S}_{L_{1}}^{\Omega}$ and $\mathbb{S}_{L_{2}}^{\Omega}$. Theorem 3.2 in [4] (or the arguments of Section 5.10) implies that for a generic choice of the Lagrangians $L_{1}, L_{2}$, and of the pseudo-gradients $X_{L_{1}}^{\Omega}, X_{L_{2}}^{\Omega}$, the space $\mu_{K}^{\Omega}\left(\gamma_{1}, \gamma_{2} ; x\right)$ - if nonempty - is a smooth manifold of dimension

$$
\operatorname{dim} \mu_{K}^{\Omega}\left(\gamma_{1}, \gamma_{2} ; x\right)=i^{\Omega}\left(\gamma_{1} ; L_{1}\right)+i^{\Omega}\left(\gamma_{2} ; L_{2}\right)-\mu^{\Omega}\left(x ; H_{1} \# H_{2}\right) .
$$

These manifolds carry coherent orientations. The energy identity is now

$$
\int_{[0,+\infty[\times[-1,1]}\left|\partial_{s} u(s, t)\right|^{2} d s d t=\mathbb{A}_{H_{1}}\left(x_{1}\right)+\mathbb{A}_{H_{2}}\left(x_{2}\right)-\mathbb{A}_{H_{1} \# H_{2}}(x),
$$

where $x_{1}(t)=u(0, t-1)$ and $x_{2}(t)=u(0, t)$. Since $\pi \circ x_{1}$ is in the unstable manifold of $\gamma_{1}$ and $\pi \circ x_{2}$ is in the unstable manifold of $\gamma_{2}$, the inequality (4-4) implies that

$$
\mathbb{A}_{H_{1}}\left(x_{1}\right) \leq \mathbb{S}_{L_{1}}\left(\gamma_{1}\right), \quad \mathbb{A}_{H_{2}}\left(x_{2}\right) \leq \mathbb{S}_{L_{2}}\left(\gamma_{2}\right),
$$

so the elements $u$ of $M_{K}^{\Omega}\left(\gamma_{1}, \gamma_{2} ; x\right)$ satisfy the energy estimate

$$
\int_{[0,+\infty[\times[-1,1]}\left|\partial_{s} u(s, t)\right|^{2} d s d t \leq \mathbb{S}_{L_{1}}\left(\gamma_{1}\right)+\mathbb{S}_{L_{2}}\left(\gamma_{2}\right)-\mathbb{A}_{H_{1} \# H_{2}}(x) .
$$

When $i^{\Omega}\left(\gamma_{1} ; L_{1}\right)+i^{\Omega}\left(\gamma_{2} ; L_{2}\right)=\mu^{\Omega}\left(x ; H_{1} \oplus H_{2}\right)$, the space $\mu_{K}^{\Omega}\left(\gamma_{1}, \gamma_{2} ; x\right)$ is a compact zero-dimensional oriented manifold. If $n_{K}^{\Omega}\left(\gamma_{1}, \gamma_{2} ; x\right)$ is the algebraic sum of its points, we can define the homomorphism

$$
\begin{aligned}
K^{\Omega}:\left(M\left(\mathbb{S}_{L_{1}}^{\Omega}\right) \otimes M\left(\mathbb{S}_{L_{2}}^{\Omega}\right)\right)_{k} & \rightarrow F_{k}^{\Omega}\left(H_{1} \# H_{2}\right), \\
\gamma_{1} \otimes \gamma_{2} & \mapsto \sum_{\substack{x \in \mathscr{P}^{\Omega}\left(H_{1} \# H_{2}\right) \\
\mu^{\Omega}(x)=k}} n_{K}^{\Omega}\left(\gamma_{1}, \gamma_{2} ; x\right) x .
\end{aligned}
$$

A standard gluing argument shows that $K^{\Omega}$ is a chain map.

It is easy to construct a homotopy $P_{K}^{\#}$ between $\Phi_{L_{1} \# L_{2}}^{\Omega} \circ M_{\#}$ and $K^{\Omega}$. In fact, it is enough to consider the space of pairs $(\alpha, u)$, where $\alpha$ is a positive number and $u$ is a solution of the Floer equation on $[0,+\infty[\times[-1,1]$ which converges to $x$ for $s \rightarrow+\infty$, and such that the curve $t \mapsto \pi \circ u(0,2 t-1)$ belongs to the evolution at time $\alpha$ of

$$
\Gamma\left(W^{u}\left(\gamma_{1} ; X_{L_{1}}^{\Omega}\right) \times W^{u}\left(\gamma_{2} ; X_{L_{2}}^{\Omega}\right)\right),
$$


by flow of $X_{L_{1} \# L_{2}}^{\Omega}$, a pseudo-gradient for $\mathbb{S}_{L_{1} \# L_{2}}^{\Omega}$. Here $\Gamma$ is the concatenation map defined in Section 1.1. More precisely, set

$$
\begin{array}{r}
\mathcal{M}_{K}^{\#}\left(\gamma_{1}, \gamma_{2} ; x\right):=\left\{(\alpha, u) \mid \alpha>0, u:\left[0,+\infty\left[\times[-1,1] \rightarrow T^{*} M \text { solves } \bar{\partial}_{J, H}(u)=0,\right.\right.\right. \\
\pi \circ u(s,-1)=\pi \circ u(s, 1)=q_{0} \forall s \geq 0, \lim _{s \rightarrow+\infty} u(s, 2 t-1)=x(t), \\
\pi \circ u(0,2 \cdot-1) \in \phi_{\alpha}^{\Omega}\left(\Gamma\left(W^{u}\left(\gamma_{1} ; X_{L_{1}}^{\Omega}\right) \times W^{u}\left(\gamma_{2} ; X_{L_{2}}^{\Omega}\right)\right)\right\},
\end{array}
$$

where $\phi_{S}^{\Omega}$ denotes the flow of $X_{L_{1} \# L_{2}}^{\Omega}$. For a generic choice of $L_{1}, L_{2}, X_{L_{1}}^{\Omega}$, $X_{L_{2}}^{\Omega}$, and $X_{L_{1} \# L_{2}}^{\Omega}$, the space $M_{K}^{\#}\left(\gamma_{1}, \gamma_{2} ; x\right)$ - if nonempty - is a smooth manifold of dimension

$$
\operatorname{dim} \mathcal{M}_{K}^{\#}\left(\gamma_{1}, \gamma_{2} ; x\right)=i^{\Omega}\left(\gamma_{1} ; L_{1}\right)+i^{\Omega}\left(\gamma_{2} ; L_{2}\right)-\mu^{\Omega}\left(x ; H_{1} \# H_{2}\right)+1,
$$

and these manifolds carry coherent orientations. The energy estimate is again (4-5). By counting the elements of the zero-dimensional manifolds, we obtain a homomorphism

$$
P_{K}^{\#}:\left(M\left(\mathbb{S}_{L_{1}}^{\Omega}\right) \otimes M\left(\mathbb{S}_{L_{2}}^{\Omega}\right)\right)_{k} \rightarrow F_{k+1}^{\Omega}\left(H_{1} \# H_{2}\right) .
$$

A standard gluing argument shows that $P_{K}^{\#}$ is a chain homotopy between $\Phi_{L_{1} \# L_{2}}^{\Omega} \circ M_{\#}$ and $K^{\Omega}$.

The homotopy $P_{\Upsilon}^{K}$ between $K^{\Omega}$ and $\Upsilon^{\Omega} \circ\left(\Phi_{L_{1}}^{\Omega} \otimes \Phi_{L_{2}}^{\Omega}\right)$ is defined by counting solutions of the Floer equation on a one-parameter family of Riemann surfaces $\Sigma_{\Upsilon}^{K}(\alpha)$, obtained by removing a point from the closed disk. More precisely, given $\alpha>0$, we define $\Sigma_{\Upsilon}^{K}(\alpha)$ as the quotient of the disjoint union $[0,+\infty[\times[-1,0] \sqcup[0,+\infty[\times[0,1]$ under the identification

$$
\left(s, 0^{-}\right) \sim\left(s, 0^{+}\right) \text {for } s \geq \alpha .
$$

This object is a Riemann surface with boundary: Its complex structure at each interior point and at each boundary point other than $(\alpha, 0)$ is induced by the inclusion, whereas the holomorphic coordinate at $(\alpha, 0)$ is given by the map

$$
\{\zeta \in \mathbb{C}|\operatorname{Re} \zeta \geq 0,| \zeta \mid<\epsilon\} \rightarrow \Sigma_{\Upsilon}^{K}(\alpha), \quad \zeta \mapsto \alpha+\zeta^{2},
$$

where the positive number $\epsilon$ is smaller than 1 and $\sqrt{\alpha}$. Given $\gamma_{1} \in \mathscr{P}\left(L_{1}\right), \gamma_{2} \in \mathscr{P}\left(L_{2}\right)$, and $x \in \mathscr{P}\left(H_{1} \# H_{2}\right)$, we consider the space of pairs $(\alpha, u)$ where $\alpha$ is a positive number, and $u(s, t)$ is a solution of the Floer equation on $\Sigma_{\Upsilon}^{K}(\alpha)$ which converges to $x$ for $s \rightarrow+\infty$, lies above some element in the unstable manifold of $\gamma_{1}$ for $s=0$ and $-1 \leq t \leq 0$, lies above some element in the unstable manifold of $\gamma_{2}$ for $s=0$ and $0 \leq t \leq 1$, and lies above $q_{0}$ at all the other boundary points. More 
precisely, $\mu_{\Upsilon}^{K}\left(\gamma_{1}, \gamma_{2} ; x\right)$ is the set of pairs $(\alpha, u)$ where $\alpha$ is a positive number and $u: \Sigma_{\Upsilon}^{K}(\alpha) \rightarrow T^{*} M$ is a solution of

$$
\bar{\partial}_{J, H}(u)=0,
$$

which satisfies the boundary conditions

$$
\begin{gathered}
\pi \circ u(s,-1)=\pi \circ u(s, 1)=q_{0}, \quad \forall s \geq 0, \\
\pi \circ u\left(s, 0^{-}\right)=\pi \circ u\left(s, 0^{+}\right)=q_{0}, \quad \forall s \in[0, \alpha], \\
\pi \circ u(0, \cdot-1) \in W^{u}\left(\gamma_{1} ; X_{L_{1}}^{\Omega}\right), \quad \pi \circ u(0, \cdot) \in W^{u}\left(\gamma_{2} ; X_{L_{2}}^{\Omega}\right),
\end{gathered}
$$

and the asymptotic condition

$$
\lim _{s \rightarrow+\infty} u(s, 2 t-1)=x(t) .
$$

The following result is proved in Section 5.10.

Proposition 4.2 For a generic choice of $L_{1}, L_{2}, X_{L_{1}}^{\Omega}$, and $X_{L_{2}}^{\Omega}, \mu_{\Upsilon}^{K}\left(\gamma_{1}, \gamma_{2} ; x\right)$ - if nonempty - is a smooth manifold of dimension

$$
\operatorname{dim} \mu_{\Upsilon}^{K}\left(\gamma_{1}, \gamma_{2} ; x\right)=i^{\Omega}\left(\gamma_{1} ; L_{1}\right)+i^{\Omega}\left(\gamma_{2} ; L_{2}\right)-\mu^{\Omega}\left(x ; H_{1} \# H_{2}\right)+1 .
$$

These manifolds carry coherent orientations.

As before, the elements $(\alpha, u)$ of $\mu_{\Upsilon}^{K}\left(\gamma_{1}, \gamma_{2} ; x\right)$ satisfy the energy estimate

$$
\int_{\Sigma_{\Upsilon}^{K}(\alpha)}\left|\partial_{s} u(s, t)\right|^{2} d s d t \leq \mathbb{S}_{L_{1}}\left(\gamma_{1}\right)+\mathbb{S}_{L_{2}}\left(\gamma_{2}\right)-\mathbb{A}_{H_{1} \# H_{2}}(x),
$$

which allows to prove compactness. By counting the zero-dimensional components, we define a homomorphism

$$
P_{\Upsilon}^{K}:\left(M\left(\mathbb{S}_{L_{1}}^{\Omega}\right) \otimes M\left(\mathbb{S}_{L_{2}}^{\Omega}\right)\right)_{k} \rightarrow F_{k+1}^{\Omega}\left(H_{1} \# H_{2}, J\right) .
$$

The conclusion arises from the following:

Proposition 4.3 The homomorphism $P_{\Upsilon}^{K}$ is a chain homotopy between $K^{\Omega}$ and $\Upsilon^{\Omega} \circ\left(\Phi_{L_{1}}^{\Omega} \otimes \Phi_{L_{2}}^{\Omega}\right)$.

The proof of the above proposition is contained in Section 6.4. It is again a compactnesscobordism argument. The analytical tool is the implicit function theorem together with a suitable family of conformal transformations of the half-strip. This concludes the proof of Theorem 4.1, hence of Theorem B. 


\subsection{A chain level proof of Theorem A}

Theorem A of the Introduction says that if $M$ is an oriented closed manifold, then there is a graded ring isomorphism

$$
H_{*}(\Lambda(M)) \cong H F_{*}^{\Lambda}\left(T^{*} M\right)
$$

where the first graded group is endowed with the loop product o (see Section 1.2), and the second one with the pair-of-pants product $H_{*} \Upsilon^{\Lambda}$ (see Section 3.3).

The loop product is the composition of two nontrivial homomorphisms: The first one is the exterior homology product $\times$ followed by the Umkehr map $e_{!}$, that is

$$
e_{!} \circ \times: H_{h}(\Lambda(M)) \otimes H_{j}(\Lambda(M)) \longrightarrow H_{h+j-n}(\Theta(M)),
$$

the second one is the homomorphism induced by concatenation,

$$
\Gamma_{*}: H_{k}(\Theta(M)) \longrightarrow H_{k}(\Lambda(M)) \text {. }
$$

In Section 2.4, we have shown how these two homomorphisms can be read on the Morse complexes of the Lagrangian action functional with either periodic or figure- 8 boundary conditions: $e_{!} \circ \times$ is induced by the chain map

$$
M_{!}: M_{h}\left(\mathbb{S}_{L_{1}}^{\Lambda}\right) \otimes M_{j}\left(\mathbb{S}_{L_{2}}^{\Lambda}\right) \longrightarrow M_{h+j-n}\left(\mathbb{S}_{L_{1} \oplus L_{2}}^{\Theta}\right),
$$

defined immediately after Proposition 2.4 , and $\Gamma_{*}$ by the chain map

$$
M_{\Gamma}: M_{k}\left(\mathbb{S}_{L_{1} \oplus L_{2}}^{\Theta}\right) \longrightarrow M_{k}\left(\mathbb{S}_{L_{1} \# L_{2}}^{\Lambda}\right),
$$

defined in Proposition 2.5. On the other hand, by Theorem 3.8 the pair-of-pants product $\Upsilon_{*}^{\Lambda}$ on the Floer homology of $T^{*} M$ with periodic boundary conditions is induced by the composition of the chain maps

$$
\begin{gathered}
E: F_{h}^{\Lambda}\left(H_{1}\right) \otimes F_{j}^{\Lambda}\left(H_{2}\right) \longrightarrow F_{h+j-n}^{\Theta}\left(H_{1} \oplus H_{2}\right), \\
G: F_{k}^{\Theta}\left(H_{1} \oplus H_{2}\right) \longrightarrow F_{k}^{\Lambda}\left(H_{1} \# H_{2}\right) .
\end{gathered}
$$

Therefore, Theorem A is an immediate consequence of the following chain level result:

Theorem 4.4 Let $L_{1}, L_{2} \in C^{\infty}(\mathbb{T} \times T M)$ be two Lagrangians which satisfy (L0) ${ }^{\Lambda}$, (L1), (L2), are such that $L_{1}(0, \cdot)=L_{2}(0, \cdot)$ with all the time derivatives, and satisfy (2-10), or equivalently (2-11). Assume also that the Lagrangian $L_{1} \# L_{2}$ defined by (2-8) satisfies (L0) ${ }^{\Lambda}$. Let $H_{1}$ and $H_{2}$ be the Fenchel transforms of $L_{1}$ and $L_{2}$, so that 
$H_{1} \# H_{2}$ is the Fenchel transform of $L_{1} \# L_{2}$, and the three Hamiltonians $H_{1}, H_{2}$, and $H_{1} \# H_{2}$ satisfy $(\mathrm{H} 0)^{\Lambda},(\mathrm{H} 1),(\mathrm{H} 2)$. Then the two squares in the diagram

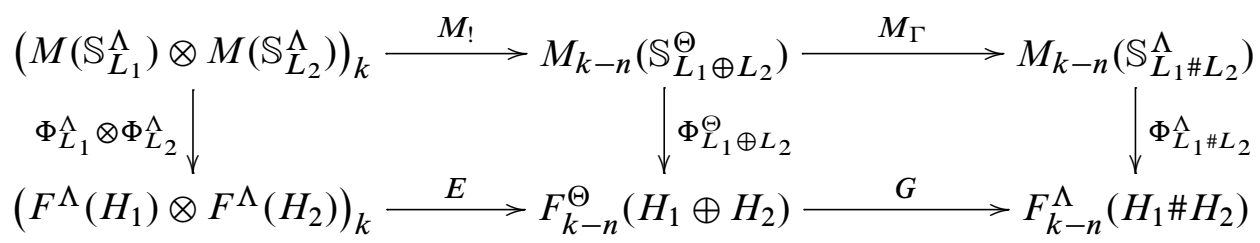

commute up to chain homotopies.

The chain homotopy commutativity of the left-hand square is more delicate and is proved in the next section. The second square is studied in Section 4.5.

\subsection{The left-hand square is homotopy commutative}

In this section we show that the chain maps $\Phi_{L_{1} \oplus L_{2}}^{\Theta} \circ M_{!}$and $E \circ\left(\Phi_{L_{1}}^{\Lambda} \otimes \Phi_{L_{2}}^{\Lambda}\right)$ are homotopic. We start by constructing a one-parameter family of chain maps

$$
K_{\alpha}^{\Lambda}:\left(M\left(\mathbb{S}_{L_{1}}^{\Lambda}\right) \otimes M\left(\mathbb{S}_{L_{2}}^{\Lambda}\right)\right)_{*} \longrightarrow F_{*-n}^{\Theta}\left(H_{1} \oplus H_{2}\right),
$$

where $\alpha$ is a nonnegative number. The definition of $K_{\alpha}^{\Lambda}$ is based on the solution spaces of the Floer equation on the Riemann surface $\Sigma_{\alpha}^{K}$ consisting of a half-cylinder with a slit. More precisely, when $\alpha$ is positive $\Sigma_{\alpha}^{K}$ is the quotient of $[0,+\infty[\times[0,1]$ by the identifications

$$
(s, 0) \sim(s, 1) \quad \forall s \in[0, \alpha] .
$$

with the holomorphic coordinate at $(\alpha, 0) \sim(\alpha, 1)$ obtained from (3-20) by a translation by $\alpha$. When $\alpha=0, \Sigma_{\alpha}^{K}=\Sigma_{0}^{K}$ is just the half-strip $\left[0,+\infty\left[\times[0,1]\right.\right.$. Fix $\gamma_{1} \in \mathscr{P}^{\Lambda}\left(L_{1}\right)$, $\gamma_{2} \in \mathscr{P}^{\Lambda}\left(L_{2}\right)$, and $x \in \mathscr{P}^{\Theta}\left(H_{1} \oplus H_{2}\right)$. Let $\mu_{\alpha}^{K}\left(\gamma_{1}, \gamma_{2} ; x\right)$ be the space of solutions $u: \Sigma_{\alpha}^{K} \rightarrow T^{*} M^{2}$ of the equation

$$
\bar{\partial}_{J, H_{1} \oplus H_{2}}(u)=0,
$$

which satisfy the boundary conditions

$$
\begin{aligned}
\pi \circ u(0, \cdot) & \in W^{u}\left(\left(\gamma_{1}, \gamma_{2}\right) ; X_{L_{1} \oplus L_{2}}^{\Lambda}\right), \\
(u(s, 0), \mathscr{b} u(s, 1)) & \in N^{*} \Delta_{M}^{(4)}, \quad \forall s \geq \alpha, \\
\lim _{s \rightarrow+\infty} u(s, t) & =x(t),
\end{aligned}
$$

where $X_{L_{1} \oplus L_{2}}^{\Lambda}$ is a pseudo-gradient for $\mathbb{S}_{L_{1} \oplus L_{2}}^{\Lambda}$ on $\Lambda^{1}(M \times M)$. Let us fix some $\alpha_{0} \geq 0$. The following result is proved in Section 5.10: 
Proposition 4.5 For a generic choice of the Lagrangians $L_{1}, L_{2}$, and of the pseudogradient $X_{L_{1} \oplus L_{2}}^{\Lambda}$, the space $\mu_{\alpha_{0}}^{K}\left(\gamma_{1}, \gamma_{2} ; x\right)$ - if nonempty - is a smooth manifold of dimension

$$
\operatorname{dim} \mu_{\alpha_{0}}^{K}\left(\gamma_{1}, \gamma_{2} ; x\right)=i^{\Lambda}\left(\gamma_{1} ; L_{1}\right)+i^{\Lambda}\left(\gamma_{2} ; L_{2}\right)-\mu^{\Theta}\left(x ; H_{1} \oplus H_{2}\right)-n .
$$

These manifolds carry coherent orientations.

Compactness is again a consequence of the energy estimate

$$
\int_{\Sigma_{\alpha}^{K}}\left|\partial_{s} u(s, t)\right|^{2} d s d t \leq \mathbb{S}_{L_{1}}\left(\gamma_{1}\right)+\mathbb{S}_{L_{2}}\left(\gamma_{2}\right)-\mathbb{A}_{H_{1} \oplus H_{2}}(x),
$$

implied by (4-4). When $i^{\Lambda}\left(\gamma_{1} ; L_{1}\right)+i^{\Lambda}\left(\gamma_{2} ; L_{2}\right)=k$ and $\mu^{\Theta}\left(x ; H_{1} \oplus H_{2}\right)=k-n$, the space $\mu_{\alpha_{0}}^{K}\left(\gamma_{1}, \gamma_{2} ; x\right)$ is a compact zero-dimensional oriented manifold. The usual counting process defines the homomorphism

$$
K_{\alpha_{0}}^{\Lambda}:\left(M\left(\mathbb{S}_{L_{1}}^{\Lambda}\right) \otimes M\left(\mathbb{S}_{L_{2}}^{\Lambda}\right)\right)_{k} \rightarrow F_{k-n}^{\Theta}\left(H_{1} \oplus H_{2}\right),
$$

and a standard gluing argument shows that $K_{\alpha_{0}}^{\Lambda}$ is a chain map.

Now assume $\alpha_{0}>0$. By standard compactness and gluing arguments, the family of solutions $\mu_{\alpha}^{K}$ for $\alpha$ varying in the interval $\left[\alpha_{0},+\infty\right.$ [ allows to define a chain homotopy between $K_{\alpha_{0}}^{\Lambda}$ and the composition $E \circ\left(\Phi_{L_{1}}^{\Lambda} \otimes \Phi_{L_{2}}^{\Lambda}\right)$.

Similarly, a compactness and cobordism argument on the Morse side shows that $K_{0}^{\Lambda}$ is chain homotopic to the composition $\Phi_{L_{1} \oplus L_{2}}^{\Theta} \circ M_{!}$. See Figure 5.

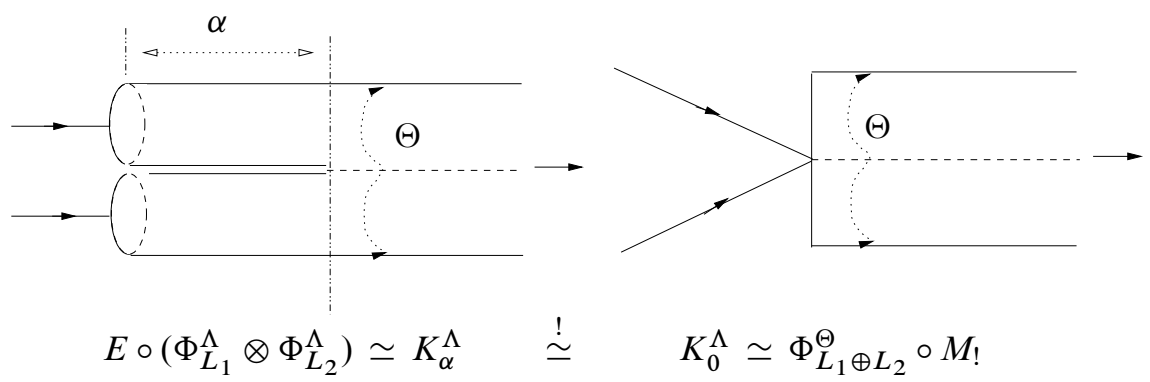

Figure 5: The homotopy through $K_{\alpha}^{\Lambda}$ and $K_{0}^{\Lambda}$

It remains to prove that $K_{\alpha_{0}}^{\Lambda}$ is homotopic to $K_{0}^{\Lambda}$. Constructing a homotopy between these chain maps by using the spaces of solutions $\mu_{\alpha}^{K}$ for $\alpha \in\left[0, \alpha_{0}\right]$ presents analytical difficulties: If we are given a solution $u$ of the limiting problem $\mu_{0}^{K}$, the existence of a (unique) one-parameter family of solutions "converging" to $u$ is problematic, because 
we do not expect $u$ to be $C^{0}$ close to the one-parameter family of solutions, due to the jump in the boundary conditions.

Therefore, we use a detour, starting from the following algebraic observation. If two chain maps $\varphi, \psi: C \rightarrow C^{\prime}$ are homotopic, so are their tensor products $\varphi \otimes \psi$ and $\psi \otimes \varphi$. The converse is obviously not true, as the example of $\varphi=0$ and $\psi$ noncontractible shows. However, it becomes true under suitable conditions on $\varphi$ and $\psi$. Denote by $(\mathbb{Z}, 0)$ the graded group which vanishes at every degree, except for degree zero, where it coincides with $\mathbb{Z}$. We see $(\mathbb{Z}, 0)$ as a chain complex with the trivial boundary operator. Then we have the following:

Lemma 4.6 Let $(C, \partial)$ and $\left(C^{\prime}, \partial\right)$ be chain complexes, bounded from below. Let $\varphi, \psi: C \rightarrow C^{\prime}$ be chain maps. Assume that there is an element $\epsilon \in C_{0}$ with $\partial \epsilon=0$ and a chain map $\delta: C^{\prime} \rightarrow(\mathbb{Z}, 0)$ such that

$$
\delta(\varphi(\epsilon))=\delta(\psi(\epsilon))=1 .
$$

If $\varphi \otimes \psi$ is homotopic to $\psi \otimes \varphi$, then $\varphi$ is homotopic to $\psi$.

Proof Let $\pi$ be the chain map

$$
\pi: C^{\prime} \otimes C^{\prime} \rightarrow C^{\prime} \otimes(\mathbb{Z}, 0) \cong C^{\prime}, \quad \pi=\mathrm{id} \otimes \delta .
$$

Let $H: C \otimes C \rightarrow C^{\prime} \otimes C^{\prime}$ be a chain homotopy between $\varphi \otimes \psi$ and $\psi \otimes \varphi$, that is

$$
\varphi \otimes \psi-\psi \otimes \varphi=\partial H+H \partial .
$$

If we define the homomorphism $h: C \rightarrow C^{\prime}$ by

$$
h(a):=\pi \circ H(a \otimes \epsilon), \quad \forall a \in C,
$$

we have

$$
\begin{aligned}
\partial h(a)+h \partial a & =\partial \pi(H(a \otimes \epsilon))+\pi(H \partial(a \otimes \epsilon)) \\
& =\pi(\partial H(a \otimes \epsilon)+H \partial(a \otimes \epsilon))=\pi(\varphi(a) \otimes \psi(\epsilon)-\psi(a) \otimes \varphi(\epsilon)) \\
& =\varphi(a) \otimes \delta(\psi(\epsilon))-\psi(a) \otimes \delta(\varphi(\epsilon))=\varphi(a)-\psi(a) .
\end{aligned}
$$

Hence $h$ is the required chain homotopy.

We shall apply the above lemma to the complexes

$$
C_{k}=\left(M\left(\mathbb{S}_{L_{1}}^{\Lambda}\right) \otimes M\left(\mathbb{S}_{L_{2}}^{\Lambda}\right)\right)_{k+n}, \quad C_{k}^{\prime}=F_{k}^{\Theta}\left(H_{1} \oplus H_{2}\right),
$$

and to the chain maps $K_{0}^{\Lambda}$ and $K_{\alpha_{0}}^{\Lambda}$. The tensor products $K_{0}^{\Lambda} \otimes K_{\alpha_{0}}^{\Lambda}$ and $K_{\alpha_{0}}^{\Lambda} \otimes K_{0}^{\Lambda}$ are represented by the coupling - in two different orders - of the corresponding elliptic boundary value problems. 
Proposition 4.7 The chain maps $K_{0}^{\Lambda} \otimes K_{\alpha_{0}}^{\Lambda}$ and $K_{\alpha_{0}}^{\Lambda} \otimes K_{0}^{\Lambda}$ are homotopic.

Constructing a homotopy between the coupled problems is easier than dealing with the original ones: we can keep $\alpha_{0}$ fixed and rotate the boundary condition on the initial part of the half-strip. This argument is similar to an alternative way, due to $\mathrm{H} \mathrm{Hofer}$, to prove the gluing statements in standard Floer homology. Details of the proof of Proposition 4.7 are contained in Section 6.5 below.

Here we just construct the cycle $\epsilon$ and the chain map $\delta$ required in Lemma 4.6. Let

$$
\tilde{\delta}: M\left(\mathbb{S}_{L_{1} \oplus L_{2}}^{\Theta}\right) \rightarrow(\mathbb{Z}, 0)
$$

be the standard augmentation on the Morse complex of the Lagrangian action functional on the space of figure- 8 loops, that is the homomorphism mapping each generator $\gamma \in \mathscr{P}^{\Theta}\left(L_{1} \oplus L_{2}\right)$ of Morse index zero into 1. Since the unstable manifold of a critical point $\gamma$ of Morse index 1 is one-dimensional, its boundary is of the form $\gamma_{1}-\gamma_{2}$, where $\gamma_{1}$ and $\gamma_{2}$ are two relative minimizers. Hence, $\tilde{\delta}$ is a chain map. We choose the chain map

$$
\delta: F^{\Theta}\left(H_{1} \oplus H_{2}\right) \rightarrow(\mathbb{Z}, 0)
$$

to be the composition of $\tilde{\delta}$ with the isomorphism $\left(\Phi_{L_{1} \oplus L_{2}}^{\Theta}\right)^{-1}$ from the Floer to the Morse complex. Since the latter isomorphism is the identity on global minimizers, $\delta(x)=1$ if $x \in \mathscr{P}^{\Theta}\left(H_{1} \oplus H_{2}\right)$ corresponds to a global minimizer of $\mathbb{S}_{L_{1} \oplus L_{2}}^{\Theta}$.

We now construct the cycle $\epsilon$ in $\left(M\left(\mathbb{S}_{L_{1}}^{\Lambda}\right) \otimes M\left(\mathbb{S}_{L_{2}}^{\Lambda}\right)\right)_{n}$. Since changing the Lagrangians $L_{1}$ and $L_{2}$ (and the corresponding Hamiltonians) changes the chain maps appearing in the diagram of Theorem 4.4 by a chain homotopy, we are free to choose the Lagrangians so to make the construction easier.

We consider a Lagrangian of the form

$$
L_{1}(t, q, v):=\frac{1}{2}|v|^{2}-V_{1}(t, q),
$$

where the potential $V_{1} \in C^{\infty}(\mathbb{T} \times M)$ satisfies

$$
V_{1}(t, q)<V_{1}\left(t, q_{0}\right)=0,
$$$$
\forall t \in \mathbb{T}, \forall q \in M \backslash\left\{q_{0}\right\},
$$

The corresponding Euler-Lagrange equation is

$$
\nabla_{t} \gamma^{\prime}(t)=-\operatorname{grad} V_{1}(t, \gamma(t)),
$$

where $\nabla_{t}$ denotes the covariant derivative along the curve $\gamma$. By (4-10) and (4-11), the constant curve $q_{0}$ is a nondegenerate minimizer for the action functional $\mathbb{S}_{L_{1}}^{\Lambda}$ on 
the free loop space (actually, it is the unique global minimizer), so

$$
i^{\Lambda}\left(q_{0} ; L_{1}\right)=0 \text {. }
$$

Notice also that the equilibrium point $\left(q_{0}, 0\right)$ is hyperbolic and unstable for the Hamiltonian dynamics on $T^{*} M$ induced by the Fenchel dual Hamiltonian $H_{1}$. We claim that there exists $\omega>0$ such that

$$
\begin{aligned}
& \text { every solution } \gamma \text { of (4-12) such that } \gamma(0)=\gamma(1) \text {, other than } \gamma(t) \equiv q_{0} \text {, } \\
& \text { satisfies } \mathbb{S}_{L_{1}}(\gamma) \geq \omega \text {. }
\end{aligned}
$$

Assuming the contrary, there exists a sequence $\left(\gamma_{h}\right)$ of solutions of (4-12) with $\gamma_{h}(0)=$ $\gamma_{h}(1)$ and $0<\mathbb{S}_{L_{1}}\left(\gamma_{h}\right) \rightarrow 0$. The space of solutions of (4-12) with action bounded from above is compact - for instance in $C^{\infty}([0,1], M)$ - so a subsequence of $\left(\gamma_{h}\right)$ converges to a solution of (4-12) with zero action. Since $q_{0}$ is the only solution with zero action, we find nonconstant solutions $\gamma$ of (4-12) with $\gamma(0)=\gamma(1)$ in any $C^{\infty}$ neighborhood of the constant curve $q_{0}$. But this is impossible: The fact that the local stable and unstable manifolds of the hyperbolic equilibrium point $\left(q_{0}, 0\right) \in T^{*} M$ are transverse to the vertical foliation $\left\{T_{q}^{*} M \mid q \in M\right\}$ easily implies that if $\left(x_{h}\right)$ is a sequence in the phase space $T^{*} M$ tending to $\left(q_{0}, 0\right)$ such that the Hamiltonian orbit of $x_{h}$ at time $T_{h}$ is on the leaf $T_{\pi\left(x_{h}\right)}^{*} M$ containing $x_{h}$, then the sequence $\left(T_{h}\right)$ must diverge.

A generic choice of the potential $V_{1}$ satisfying (4-10) and (4-11) produces a Lagrangian $L_{1}$ whose associated action functional is Morse on $\Lambda^{1}(M)$.

Next we consider an autonomous Lagrangian of the form

$$
\tilde{L}_{2}(q, v):=\frac{1}{2}|v|^{2}-V_{2}(q),
$$

where:

(i) $V_{2}$ is a smooth Morse function on $M$.

(ii) $0=V_{2}\left(q_{0}\right)<V_{2}(q)<\omega / 2$ for every $q \in M \backslash\left\{q_{0}\right\}$.

(iii) $V_{2}$ has no local minimizers other than $q_{0}$.

(iv) $\left\|V_{2}\right\|_{C^{2}(M)}<\rho$.

Here $\rho$ is a small positive constant, whose size is to be specified. The critical points of $V_{2}$ are equilibrium solutions of the Euler-Lagrange equation associated to $\widetilde{L}_{2}$. The second differential of the action at such an equilibrium solution $q$ is

$$
d^{2} \mathbb{S}_{\widetilde{L}_{2}}^{\Lambda}(q)[\xi, \xi]=\int_{0}^{1}\left(\left\langle\xi^{\prime}(t), \xi^{\prime}(t)\right\rangle-\left\langle\operatorname{Hess} V_{2}(q) \xi(t), \xi(t)\right\rangle\right) d t
$$


If $0<\rho<2 \pi$, (iv) implies that $q$ is a nondegenerate critical point of $\mathbb{S} \widetilde{L}_{2}$ with Morse index

$$
i^{\Lambda}\left(q ; \tilde{L}_{2}\right)=n-i\left(q ; V_{2}\right),
$$

a maximal negative subspace being the space of constant vector fields at $q$ taking values into the positive eigenspace of Hess $V_{2}(q)$.

The infimum of the energy

$$
\frac{1}{2} \int_{0}^{1}\left|\gamma^{\prime}(t)\right|^{2} d t
$$

over all nonconstant closed geodesics is positive. It follows that if $\rho$ in (iv) is small enough, then

$$
\inf \left\{\mathbb{S}_{\widetilde{L}_{2}}(\gamma) \mid \gamma \in \mathscr{P}^{\Lambda}\left(\widetilde{L}_{2}\right), \gamma \text { nonconstant }\right\}>0 .
$$

Since the Lagrangian $\widetilde{L}_{2}$ is autonomous, nonconstant periodic orbits cannot be nondegenerate critical points of $\mathbb{S}_{\widetilde{L}_{2}}^{\Lambda}$. Let $W \in C^{\infty}(\mathbb{T} \times M)$ be a $C^{2}$-small time-dependent potential satisfying:

(v) $0 \leq W(t, q)<\omega / 2$ for every $(t, q) \in \mathbb{T} \times M$.

(vi) $W(t, q)=0, \operatorname{grad} W(t, q)=0$, Hess $W(t, q)=0$ for every $t \in \mathbb{T}$ and every critical point $q$ of $V_{2}$.

For a generic choice of such a $W$, the action functional associated to the Lagrangian

$$
L_{2}(t, q, v):=\frac{1}{2}\langle v, v\rangle-V_{2}(q)-W(t, q),
$$

is Morse on $\Lambda^{1}(M)$. By (vi), the critical points of $V_{2}$ are still equilibrium solutions of the Euler-Lagrange equation

$$
\begin{aligned}
\nabla_{t} \gamma^{\prime}(t) & =-\operatorname{grad}\left(V_{2}(t, \gamma(t))+W(t, \gamma(t)),\right. \\
i^{\Lambda}\left(q ; L_{2}\right) & =n-i\left(q ; V_{2}\right) \quad \forall q \in \operatorname{crit} V_{2} .
\end{aligned}
$$

Moreover, (4-14) implies that if the $C^{2}$ norm of $W$ is small enough, then

$$
\inf \left\{\mathbb{S}_{L_{2}}(\gamma) \mid \gamma \in \mathscr{P}^{\Lambda}\left(L_{2}\right), \gamma \text { nonconstant }\right\}>0 .
$$

Up to a generic perturbation of the potential $W$, we may also assume that the equilibrium solution $q_{0}$ is the only 1 -periodic solution of (4-15) with $\gamma(0)=\gamma(1)=q_{0}$ (generically, the set of periodic orbits is discrete, and so is the set of their initial points).

Since the inclusion c: $M \hookrightarrow \Lambda^{1}(M)$ induces an injective homomorphism between the singular homology groups, the image $\mathrm{c}_{*}([M])$ of the fundamental class of the oriented closed manifold $M$ does not vanish in $\Lambda^{1}(M)$. By (ii) and (v), the action 
$\mathbb{S}_{L_{2}}$ of every constant curve in $M$ does not exceed 0 . So we can regard $\mathrm{c}_{*}([M])$ as a non vanishing element of the homology of the sublevel $\left\{\mathbb{S}_{L_{2}}^{\Lambda} \leq 0\right\}$. The singular homology of $\left\{\mathbb{S}_{L_{2}}^{\Lambda} \leq 0\right\}$ is isomorphic to the homology of the subcomplex of the Morse complex $M_{*}\left(\mathbb{S}_{L_{2}}^{\Lambda}\right)$ generated by the critical points of $\mathbb{S}_{L_{2}}^{\Lambda}$ whose action does not exceed 0. By (4-17), these critical points are the equilibrium solutions $q$, with $q \in$ crit $\left(V_{2}\right)$. By (ii), (iii), and (4-16), the only critical point of index $n$ in this sublevel is $q_{0}$. It follows that the Morse homological counterpart of $\mathrm{c}_{*}([M])$ is $\pm q_{0}$. In particular, $q_{0} \in M_{n}\left(\mathbb{S}_{L_{2}}^{\Lambda}\right)$ is a cycle. Since $\mathbb{S}_{L_{2}}^{\Lambda}\left(q_{0}\right)=0$,

$$
\mathbb{S}_{L_{2}}^{\Lambda}(\gamma) \leq 0, \quad \forall \gamma \in W^{u}\left(q_{0} ; X_{L_{2}}^{\Lambda}\right)
$$

We now regard the pair $\left(q_{0}, q_{0}\right)$ as an element of $\mathscr{P}^{\Theta}\left(L_{1} \oplus L_{2}\right)$. We claim that if $\rho$ is small enough, (iv) implies that $\left(q_{0}, q_{0}\right)$ is a nondegenerate minimizer for $\mathbb{S}_{L_{1} \oplus L_{2}}$ on the space of figure-8 loops $\Theta^{1}(M)$. The second differential of $\mathbb{S}_{L_{1} \oplus L_{2}}^{\Theta}$ at $\left(q_{0}, q_{0}\right)$ is the quadratic form

$$
\begin{aligned}
d^{2} \mathbb{S}_{L_{1} \oplus L_{2}}^{\Theta}\left(q_{0}, q_{0}\right)\left[\left(\xi_{1}, \xi_{2}\right)\right]^{2} & \\
& =\int_{0}^{1}\left(\left\langle\xi_{1}^{\prime}, \xi_{1}^{\prime}\right\rangle-\left\langle\operatorname{Hess} V_{1}\left(t, q_{0}\right) \xi_{1}, \xi_{1}\right\rangle+\left\langle\xi_{2}^{\prime}, \xi_{2}^{\prime}\right\rangle-\left\langle\operatorname{Hess} V_{2}\left(q_{0}\right) \xi_{2}, \xi_{2}\right\rangle\right) d t
\end{aligned}
$$

on the space of curves $\left(\xi_{1}, \xi_{2}\right)$ in the Sobolev space $W^{1,2}\left([0,1], T_{q_{0}} M \times T_{q_{0}} M\right)$ which satisfy the boundary conditions

$$
\xi_{1}(0)=\xi_{1}(1)=\xi_{2}(0)=\xi_{2}(1) .
$$

By (4-11), we can find $\alpha>0$ such that

$$
\text { Hess } V_{1}\left(t, q_{0}\right) \leq-\alpha I \text {. }
$$

By comparison, it is enough to show that the quadratic form

$$
Q_{\rho}\left(u_{1}, u_{2}\right):=\int_{0}^{1}\left(u_{1}^{\prime}(t)^{2}+\alpha u_{1}(t)^{2}+u_{2}^{\prime}(t)^{2}-\rho u_{2}(t)^{2}\right) d t
$$

is coercive on the space

$$
\left\{\left(u_{1}, u_{2}\right) \in W^{1,2}\left([0,1], \mathbb{R}^{2}\right) \mid u_{1}(0)=u_{1}(1)=u_{2}(0)=u_{2}(1)\right\} .
$$


When $\rho=0$, the quadratic form $Q_{0}$ is nonnegative. An isotropic element $\left(u_{1}, u_{2}\right)$ for $Q_{0}$ would solve the boundary value problem

$$
\begin{aligned}
-u_{1}^{\prime \prime}(t)+\alpha u_{1}(t) & =0, \\
-u_{2}^{\prime \prime}(t) & =0, \\
u_{1}(0)=u_{1}(1) & =u_{2}(0)=u_{2}(1), \\
u_{1}^{\prime}(1)-u_{1}^{\prime}(0) & =u_{2}^{\prime}(0)-u_{2}^{\prime}(1) .
\end{aligned}
$$

By (4-20) and (4-21), $u_{2}$ is constant, so by (4-21) and (4-22) $u_{1}$ is a periodic solution of (4-19). Since $\alpha$ is positive, $u_{1}$ is zero and by (4-21) so is $u_{2}$. Since the bounded self-adjoint operator associated to $Q_{0}$ is Fredholm, we deduce that $Q_{0}$ is coercive. By continuity, $Q_{\rho}$ remains coercive for $\rho$ small. This proves our claim.

Let $H_{1}$ and $H_{2}$ be the Hamiltonians which are Fenchel dual to $L_{1}$ and $L_{2}$. In order to simplify the notation, let us denote by $\left(q_{0}, q_{0}\right)$ also the constant curve in $T^{*} M^{2}$ identically equal to $\left(\left(q_{0}, 0\right),\left(q_{0}, 0\right)\right)$. Then $\left(q_{0}, q_{0}\right)$ is a nondegenerate element of $\mathscr{P}^{\Theta}\left(H_{1} \oplus H_{2}\right)$, and it has Maslov index

$$
\mu^{\Theta}\left(q_{0}, q_{0}\right)=i^{\Theta}\left(q_{0}, q_{0}\right)=0 .
$$

Let $x$ be an element in $\mathscr{P}^{\Theta}\left(H_{1} \oplus H_{2}\right)$, and let $\gamma$ be its projection onto $M \times M$. By the definition of the Euler-Lagrange problem for figure-8 loops (see in particular the boundary conditions (2-4)), $\gamma_{1}$ is a solution of (4-12), $\gamma_{2}$ is a solution of (4-15), and

$$
\gamma_{1}(0)=\gamma_{1}(1)=\gamma_{2}(0)=\gamma_{2}(1), \quad \gamma_{2}^{\prime}(1)-\gamma_{2}^{\prime}(0)=\gamma_{1}^{\prime}(0)-\gamma_{1}^{\prime}(1) .
$$

If $\gamma_{1}$ is the constant orbit $q_{0}$, then (4-23) implies that $\gamma_{2}$ is a 1-periodic solution of (4-15) such that $\gamma_{2}(0)=\gamma_{2}(1)=q_{0}$, and we have assumed that the only curve with these properties is $\gamma_{2} \equiv q_{0}$. If $\gamma_{1}$ is not the constant orbit $q_{0},(4-13)$ implies that

$$
\mathbb{S}_{L_{1}}\left(\gamma_{1}\right) \geq \omega \text {. }
$$

By (ii) and (v), the infimum of $\mathbb{S}_{L_{2}}$ is larger than $-\omega$, so we deduce that

$$
\begin{aligned}
\mathbb{A}_{H_{1} \oplus H_{2}}(x)=\mathbb{A}_{H_{1}}\left(x_{1}\right)+\mathbb{A}_{H_{2}}\left(x_{2}\right)=\mathbb{S}_{L_{1}}\left(\gamma_{1}\right)+\mathbb{S}_{L_{2}}\left(\gamma_{2}\right)>0, & \\
& \forall x \in \mathscr{P}^{\Theta}\left(H_{1} \oplus H_{2}\right) \backslash\left\{\left(q_{0}, q_{0}\right)\right\},
\end{aligned}
$$

so $\left(q_{0}, q_{0}\right)$ is the global minimizer of $\mathbb{S}_{L_{1} \oplus L_{2}}^{\Theta}$.

Now we choose $\epsilon$ in the $n$-th degree component of the chain complex $M\left(\mathbb{S}_{L_{1}}^{\Lambda}\right) \otimes$ $M\left(\mathbb{S}_{L_{2}}^{\Lambda}\right)$ to be the cycle

$$
\epsilon=q_{0} \otimes q_{0} \in M_{0}\left(\mathbb{S}_{L_{1}}^{\Lambda}\right) \otimes M_{n}\left(\mathbb{S}_{L_{2}}^{\Lambda}\right) .
$$


We must show that

$$
\delta\left(K_{0}^{\Lambda}\left(q_{0} \otimes q_{0}\right)\right)=\delta\left(K_{\alpha_{0}}^{\Lambda}\left(q_{0} \otimes q_{0}\right)\right)=1
$$

Let $x \in \mathscr{P}^{\Theta}\left(H_{1} \oplus H_{2}\right)$, and let $u$ be an element of either

$$
\mu_{0}^{K}\left(q_{0}, q_{0} ; x\right) \quad \text { or } \quad \mu_{\alpha_{0}}^{K}\left(q_{0}, q_{0} ; x\right) .
$$

By the boundary condition (4-6), the curve $u(0, \cdot)$ projects onto a closed curve in $M \times M$ whose first component is the constant $q_{0}$ and whose second component is in the unstable manifold of $q_{0}$ with respect to the negative pseudo-gradient flow of $\mathbb{S}_{L_{2}}^{\Lambda}$. By the fundamental inequality (4-4) between the Hamiltonian and the Lagrangian action and by (4-18), we have

$$
\begin{aligned}
\mathbb{A}_{H_{1} \oplus H_{2}}(x) \leq \mathbb{A}_{H_{1} \oplus H_{2}}(u(0, \cdot)) & \leq \mathbb{S}_{L_{1} \oplus L_{2}}(\pi \circ u(0, \cdot)) \\
& =\mathbb{S}_{L_{1}}^{\Lambda}\left(q_{0}\right)+\mathbb{S}_{L_{2}}^{\Lambda}\left(\pi \circ u_{2}(0, \cdot)\right) \leq 0
\end{aligned}
$$

By (4-24), $x$ must be the constant curve $\left(q_{0}, q_{0}\right)$, and all the inequalities in the above estimate are equalities. It follows that $u$ is constant, $u(s, t) \equiv\left(q_{0}, q_{0}\right)$.

Therefore, the spaces $\mu_{0}^{K}\left(q_{0}, q_{0} ; x\right)$ and $\mu_{\alpha_{0}}^{K}\left(q_{0}, q_{0} ; x\right)$ are nonempty if and only if $x=\left(q_{0}, q_{0}\right)$, and in the latter situation they consist of the unique constant solution $u \equiv\left(q_{0}, q_{0}\right)$. Automatic transversality holds for such solutions (see [4, Proposition 3.7]), so such a picture survives to the generic perturbations of $L_{1}, L_{2}$, and of the pseudo-gradients which are necessary to achieve a Morse-Smale situation. Taking also the orientations into account, it follows that

$$
K_{0}^{\Lambda}\left(q_{0} \otimes q_{0}\right)=\left(q_{0}, q_{0}\right), \quad K_{\alpha}^{\Lambda}\left(q_{0} \otimes q_{0}\right)=\left(q_{0}, q_{0}\right) .
$$

Since $\left(q_{0}, q_{0}\right)$ is the global minimizer of $\mathbb{S}_{L_{1} \oplus L_{2}}^{\Theta} \delta\left(\left(q_{0}, q_{0}\right)\right)=1$ as previously observed, so (4-25) holds.

This concludes the construction of a cycle $\epsilon$ and a chain map $\delta$ which satisfy the assumptions of Lemma 4.6. Together with Proposition 4.7, this proves that left-hand square in the diagram of Theorem 4.4 commutes up to a chain homotopy.

\subsection{The right-hand square is homotopy commutative}

In this section we prove that the chain maps $\Phi_{L_{1} \# L_{2}}^{\Lambda} \circ M_{\Gamma}$ and $G \circ \Phi_{L_{1} \oplus L_{2}}^{\Theta}$ are both homotopic to a third chain map, named $K^{\Theta}$. This fact implies that the right-hand square in the diagram of Theorem 4.4 commutes up to chain homotopy.

The chain map $K^{\Theta}$ is defined by using the following spaces of solutions of the Floer equation on the half-cylinder for the Hamiltonian $H_{1} \# H_{2}$ : given $\gamma \in \mathscr{P}^{\Theta}\left(L_{1} \oplus L_{2}\right)$ 
and $x \in \mathscr{P}^{\Lambda}\left(H_{1} \# H_{2}\right)$, set

$$
\begin{aligned}
\mu_{K}^{\Theta}(\gamma ; x):=\{u:[0,+\infty[\times \mathbb{T} & \rightarrow T^{*} M \mid \bar{\partial}_{J, H_{1} \# H_{2}}(u)=0, \\
& \left.\pi \circ u(0, \cdot) \in \Gamma\left(W^{u}\left(\gamma ; X_{L_{1} \oplus L_{2}}^{\Theta}\right)\right), \lim _{s \rightarrow+\infty} u(s, \cdot)=x\right\},
\end{aligned}
$$

where $X_{L_{1} \oplus L_{2}}^{\Theta}$ is a pseudo-gradient for $\mathbb{S}_{L_{1} \oplus L_{2}}^{\Theta}$ on $\Theta^{1}(M)$. By Theorem 3.2 in [4] (or by the arguments of Section 5.10), the space $\mu_{K}^{\Theta}(\gamma ; x)$ is a smooth manifold of dimension

$$
\operatorname{dim} \mu_{K}^{\Theta}(\gamma ; x)=i^{\Theta}(\gamma)-\mu^{\Lambda}(x),
$$

for a generic choice of $L_{1}, L_{2}$, and $X_{L_{1} \oplus L_{2}}^{\Theta}$. These manifolds carry coherent orientations.

Compactness follows from the energy estimate

$$
\int_{[0,+\infty[\times \mathbb{T}}\left|\partial_{s} u(s, t)\right|^{2} d s d t \leq \mathbb{S}_{L_{1} \oplus L_{2}}(\gamma)-\mathbb{A}_{H_{1} \# H_{2}}(x),
$$

which is implied by (4-4). By counting the elements of the zero-dimensional spaces, we define a chain map

$$
K^{\Theta}: M_{j}\left(\mathbb{S}_{L_{1} \oplus L_{2}}^{\Theta}\right) \rightarrow F_{j}^{\Lambda}\left(H_{1} \# H_{2}\right) .
$$

It is easy to construct a chain homotopy $P_{K}^{\Gamma}$ between $\Phi_{L_{1} \# L_{2}}^{\Lambda} \circ M_{\Gamma}$ and $K^{\Theta}$ by considering the space

$$
\begin{aligned}
\mu_{K}^{\Gamma}(\gamma ; x):=\{(\alpha, u) \mid & \alpha>0, u:\left[0,+\infty\left[\times \mathbb{T} \rightarrow T^{*} M, \bar{\partial}_{J, H_{1} \# H_{2}}(u)=0,\right.\right. \\
& \left.\phi_{-\alpha}^{\Lambda}(\pi \circ u(0, \cdot)) \in \Gamma\left(W^{u}\left(\gamma ; X_{L_{1} \oplus L_{2}}^{\Theta}\right)\right), \lim _{s \rightarrow+\infty} u(s, \cdot)=x\right\} .
\end{aligned}
$$

where $\phi_{S}^{\Lambda}$ denotes the flow of $X_{L_{1} \# L_{2}}^{\Lambda}$ on $\Lambda^{1}(M)$. As before, we find that generically $\mu_{K}^{\Gamma}\left(\gamma_{1}, \gamma_{2} ; x\right)$ is a manifold of dimension

$$
\operatorname{dim} \mu_{K}^{\Gamma}(\gamma ; x)=i^{\Lambda}(\gamma)-\mu^{\Theta}(x)+1 .
$$

Compactness holds, so an algebraic count of the zero-dimensional spaces produces the homomorphism

$$
P_{K}^{\Gamma}: M_{j}\left(\mathbb{S}_{L_{1} \oplus L_{2}}^{\Theta}\right) \rightarrow F_{j+1}^{\Lambda}\left(H_{1} \# H_{2}\right) .
$$

A standard gluing argument shows that $P_{K}^{\Gamma}$ is the required homotopy.

Finally, the construction of the chain homotopy $P_{G}^{K}$ between $K^{\Theta}$ and $G \circ \Phi_{L_{1} \oplus L_{2}}^{\Theta}$ is based on the one-parameter family of Riemann surfaces $\Sigma_{G}^{K}(\alpha), \alpha>0$, defined 
as the quotient of the disjoint union $[0,+\infty[\times[-1,0] \sqcup[0,+\infty[\times[0,1]$ under the identifications

$$
\left(s, 0^{-}\right) \sim\left(s, 0^{+}\right) \text {and }(s,-1) \sim(s, 1) \text { for } s \geq \alpha .
$$

This object is a Riemann surface with boundary, the holomorphic structure at $(\alpha, 0)$ being given by the map

$$
\{\zeta \in \mathbb{C}|\operatorname{Re} \zeta \geq 0,| \zeta \mid<\epsilon\} \rightarrow \Sigma_{G}^{K}(\alpha), \quad \zeta \mapsto \alpha+\zeta^{2},
$$

and the holomorphic structure at $(\alpha,-1) \sim(\alpha, 1)$ being given by the map

$$
\{\zeta \in \mathbb{C}|\operatorname{Re} \zeta \geq 0,| \zeta \mid<\epsilon\} \rightarrow \Sigma_{G}^{K}(\alpha), \quad \zeta \mapsto \begin{cases}\alpha-i+\zeta^{2} & \text { if } \operatorname{Im} \zeta \geq 0, \\ \alpha+i+\zeta^{2} & \text { if } \operatorname{Im} \zeta \leq 0 .\end{cases}
$$

Here $\epsilon$ is a positive number smaller than 1 and $\sqrt{\alpha}$.

Given $\gamma \in \mathscr{P}^{\Theta}\left(L_{1} \oplus L_{2}\right)$ and $x \in \mathscr{P}^{\Lambda}\left(H_{1} \# H_{2}\right)$, we consider the space $\mathcal{M}_{G}^{K}(\gamma, x)$ of pairs $(\alpha, u)$ where $\alpha$ is a positive number and $u$ : $\Sigma_{G}^{K}(\alpha) \rightarrow T^{*} M$ solves the equation

$$
\bar{\partial}_{J, H_{1} \# H_{2}}(u)=0,
$$

satisfies the boundary conditions

$$
\begin{gathered}
\left\{\begin{array}{l}
\pi \circ u(s,-1)=\pi \circ u\left(s, 0^{-}\right)=\pi \circ u\left(s, 0^{+}\right)=\pi \circ u(s, 1), \\
u\left(s, 0^{-}\right)-u(s,-1)+u(s, 1)-u\left(s, 0^{+}\right)=0,
\end{array} \quad \forall s \in[0, \alpha],\right. \\
(\pi \circ u(0, \cdot-1), \pi \circ u(0, \cdot)) \in W^{u}\left(\gamma, X_{L_{1} \oplus L_{2}}^{\Theta},\right.
\end{gathered}
$$

and the asymptotic condition

$$
\lim _{s \rightarrow+\infty} u(s, 2 t-1)=x(t) .
$$

The following result is proved in Section 5.10.

Proposition 4.8 For a generic choice of $L_{1}, L_{2}$, and $X_{L_{1} \oplus L_{2}}^{\Theta}, \mu_{G}^{K}(\gamma, x)-$ if nonempty - is a smooth manifold of dimension

$$
\operatorname{dim} \mu_{G}^{K}(\gamma, x)=i^{\Theta}\left(\gamma ; L_{1} \oplus L_{2}\right)-\mu^{\Lambda}\left(x ; H_{1} \# H_{2}\right)+1 .
$$

The projection $(\alpha, u) \mapsto \alpha$ is smooth on $\mu_{G}^{K}(\gamma, x)$. These manifolds carry coherent orientations.

The elements $(\alpha, u)$ of $\mu_{G}^{K}(\gamma, x)$ satisfy the energy estimate

$$
\int_{\Sigma_{G}^{K}(\alpha)}\left|\partial_{s} u(s, t)\right|^{2} d s d t \leq \mathbb{S}_{L_{1} \oplus L_{2}}(\gamma)-\mathbb{A}_{H_{1} \# H_{2}}(x) .
$$


This provides us with the compactness which is necessary to define the homomorphism

$$
P_{G}^{K}: M_{j}\left(\mathbb{S}_{L_{1} \oplus L_{2}}^{\Theta}\right) \longrightarrow F_{j+1}^{\Lambda}\left(H_{1} \# H_{2}\right),
$$

by the usual counting procedure applied to the spaces $M_{G}^{K}$. A standard gluing argument shows that $P_{G}^{K}$ is a chain homotopy between $K^{\Theta}$ and $G \circ \Phi_{L_{1} \oplus L_{2}}^{\Theta}$.

This concludes the proof of Theorem 4.4, hence of its corollary, Theorem A of the Introduction.

\subsection{Comparison between $\mathrm{C}, \mathrm{Ev}, \mathrm{I}_{\text {! }}$ and c, ev, i!}

The aim of this section is to prove that the homomorphisms

$$
\begin{aligned}
\mathrm{c}_{*}: H_{j}(M) & \rightarrow H_{j}(\Lambda(M)), \\
\mathrm{ev}_{*}: H_{j}(\Lambda(M)) & \rightarrow H_{j}(M), \\
\mathrm{i}_{!}: H_{j}(\Lambda(M)) & \rightarrow H_{j-n}\left(\Omega\left(M, q_{0}\right)\right),
\end{aligned}
$$

on the topological side (see Section 1.3), correspond - via the isomorphisms of Section 4.1 - to the homomorphisms

$$
\begin{aligned}
\mathrm{C}_{*}: H M_{j}(f) & \rightarrow H F_{j}^{\Lambda}\left(T^{*} M\right), \\
\left.\operatorname{Ev}_{*}: H F_{j}^{\Lambda}\left(T^{*} M\right)\right) & \rightarrow H M_{j}(f), \\
\mathrm{I}_{!}: H F_{j}^{\Lambda}\left(T^{*} M\right) & \rightarrow H F_{j-n}^{\Omega}\left(T^{*} M\right),
\end{aligned}
$$

on the Floer side (see Section 3.5). We start by comparing the first two pairs of homomorphisms.

Comparison between c, ev and C, Ev In Section 2.2 we have shown that the homomorphisms $\mathrm{c}_{*}$ and $\mathrm{ev}_{*}$ are induced by chain maps

$$
M \mathrm{c}: M_{j}(f) \rightarrow M_{j}\left(\mathbb{S}_{L}^{\Lambda}\right), \quad M \mathrm{ev}: M_{j}\left(\mathbb{S}_{L}^{\Lambda}\right) \rightarrow M_{j}(f),
$$

between the Morse complex of the Morse functions $f: M \rightarrow \mathbb{R}$ and $\mathbb{S}_{L}^{\Lambda}: \Lambda^{1}(M) \rightarrow \mathbb{R}$. Therefore, the fact that $c_{*}$ and $\mathrm{ev}_{*}$ correspond to $\mathrm{C}_{*}$ and $\mathrm{Ev}_{*}$ is implied by the following chain level result:

Theorem 4.9 The triangles

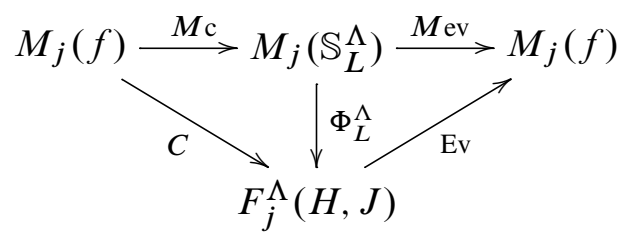

are chain homotopy commutative. 
A homotopy $P^{\mathrm{C}}$ between $\mathrm{C}$ and $\Phi_{L}^{\Lambda} \circ M \mathrm{c}$ is defined by the following spaces: Given $x \in \operatorname{crit}(f)$ and $y \in \mathscr{P}^{\Lambda}(H)$, set

$$
\begin{aligned}
\mu_{P}^{\mathrm{C}}(x, y):=\left\{(\alpha, u) \mid \alpha>0, u:\left[0,+\infty\left[\times \mathbb{T} \rightarrow T^{*} M, \bar{\partial}_{J, H}(u)=0,\right.\right.\right. & \left.\phi_{-\alpha}^{\Lambda}(\pi \circ u(0, \cdot)) \equiv q \in W^{u}(x)\right\},
\end{aligned}
$$

where $\phi^{\Lambda}$ is the flow of $X_{L}^{\Lambda}$, a pseudo-gradient for $\mathbb{S}_{L}^{\Lambda}$ on $\Lambda^{1}(M)$, and $W^{u}(x) \subset M$ is the unstable manifold of $x$ with respect to the negative gradient flow of $f$.

Similarly, the definition of the homotopy $P^{\mathrm{Ev}}$ between $\operatorname{Ev} \circ \Phi_{L}^{\Lambda}$ and $M$ ev is obtained from the composition of three homotopies based on the following spaces: Given $\gamma \in \mathscr{P}^{\Lambda}(L)$ and $x \in \operatorname{crit}(f)$, set

$$
\begin{aligned}
\mathcal{M}_{P_{1}}^{\mathrm{Ev}}(\gamma, x):= & \left\{(\alpha, u) \mid \alpha \in\left[1,+\infty\left[, u:[0, \alpha] \times \mathbb{T} \rightarrow T^{*} M \text { solves } \bar{\partial}_{J, H}(u)=0,\right.\right.\right. \\
& \left.u(\alpha, t) \in \mathbb{O}_{M} \forall t \in \mathbb{T}, u(\alpha, 0) \in W^{s}(x), \pi \circ u(0, \cdot) \in W^{u}\left(\gamma ; X_{L}^{\Lambda}\right)\right\}, \\
\mathcal{M}_{P_{2}}^{\mathrm{Ev}}(\gamma, x):= & \left\{(\alpha, u) \mid \alpha \in[0,1], u:[0,1] \times \mathbb{T} \rightarrow T^{*} M \text { solves } \bar{\partial}_{J, H}(u)=0,\right. \\
& \left.u(1, t) \in \mathbb{O}_{M} \forall t \in \mathbb{T}, u(\alpha, 0) \in W^{s}(x), \pi \circ u(0, \cdot) \in W^{u}\left(\gamma ; X_{L}^{\Lambda}\right)\right\}, \\
\mathcal{M}_{P_{3}}^{\mathrm{Ev}}(\gamma, x):= & \{(\alpha, u) \mid \alpha \in] 0,1], u:[0, \alpha] \times \mathbb{T} \rightarrow T^{*} M \text { solves } \bar{\partial}_{J, H}(u)=0, \\
& \left.u(\alpha, t) \in \mathbb{O}_{M} \forall t \in \mathbb{T}, u(0,0) \in W^{s}(x), \pi \circ u(0, \cdot) \in W^{u}\left(\gamma ; X_{L}^{\Lambda}\right)\right\} .
\end{aligned}
$$

Moreover, recalling that the definition of $M \mathrm{ev}$ is based on the space

$$
\mu_{M \mathrm{ev}}(\gamma, x)=W^{u}\left(\gamma ; X_{L}^{\Lambda}\right) \cap \mathrm{ev}^{-1}\left(W^{s}(x ;-\operatorname{grad} f)\right),
$$

we make the following observation:

Proposition 4.10 For every $\gamma \in \mathscr{P P}^{\Lambda}(L)$ and $x \in \operatorname{crit}(f)$ with $i^{\Lambda}(\gamma)=i(x ; f)$, there exists $\alpha_{o}>0$ such that for each $c$ in the finite set $M_{M \text { ev }}(\gamma, x)$ and $\alpha \in\left(0, \alpha_{o}\right]$ the problem

$$
\begin{aligned}
& u \in[0, \alpha] \times \mathbb{T} \rightarrow T^{*} M, \quad \bar{\partial}_{J, H} u=0, \\
& u(\alpha, t) \in \mathbb{O}_{M} \forall t \in \mathbb{T}, \quad \pi \circ u(0, \cdot)=c,
\end{aligned}
$$

has a unique solution with the same coherent orientation as $c$.

Proof We give a sketch of the proof, details are left to the reader.

First, given a sequence $\alpha_{n} \rightarrow 0$ and associated solutions $u_{n}:\left[0, \alpha_{n}\right] \times \mathbb{T} \rightarrow T^{*} M$ of (4-26), one can show that $u_{n} \rightarrow(c, 0) \in \Lambda\left(T^{*} M\right)$ uniformly. Here, it is important 
to make a case distinction for the three cases of possible gradient blow-up: if we set $R_{n}:=\left\|\nabla u_{n}\right\|_{\infty}=\left|\nabla u_{n}\left(z_{n}\right)\right|$, up to a subsequence we may assume that the sequence $\left(\alpha_{n} R_{n}\right)$ either is infinitesimal, or diverges, or converges to some $\kappa>0$.

The most interesting case is $\alpha_{n} R_{n} \rightarrow \kappa>0$ which is dealt with by rescaling $v_{n}=$ $u_{n}\left(\alpha_{n} \cdot \alpha_{n} \cdot\right)$ as in the proof of Lemma 6.6.

For the converse, we need a Newton type method which is hard to implement for the shrinking domains $[0, \alpha] \times \mathbb{T}$ with $\alpha \rightarrow 0$. Instead, we consider the conformally rescaled equivalent problem. Let $v(s, t)=u(\alpha s, \alpha t)$ and consider the corresponding problem for $\alpha \rightarrow 0$,

$$
\begin{aligned}
& v:[0,1] \times \mathbb{T}_{\alpha^{-1}} \rightarrow T^{*} M, \quad \bar{\partial}_{J, H_{\alpha}} v=0, \\
& \pi(v(0, t))=c(\alpha t), v(1, t) \in \mathbb{O}_{M} \forall t \in \mathbb{T}_{\alpha^{-1}},
\end{aligned}
$$

where $H_{\alpha}(t, \cdot)=\alpha H(\alpha t, \cdot)$ and $\mathbb{T}_{\alpha^{-1}}=\mathbb{R} / \alpha^{-1} \mathbb{Z}$. The proof is now based on the Newton method which requires to show that:

(a) For $v_{o}(s, t)=0 \in T_{c(\alpha t)}^{*} M$ we have $\bar{\partial}_{J, H}\left(v_{o}\right) \rightarrow 0$ as $\alpha \rightarrow 0$, which is obvious.

(b) The linearization $D_{\alpha}$ of $\bar{\partial}_{J, H_{\alpha}}$ at $v_{o}$ is invertible for small $\alpha>0$ with uniform bound on $\left\|D_{\alpha}^{-1}\right\|$ as $\alpha \rightarrow 0$.

We sketch now the proof of this uniform bound.

After suitable trivializations, the linearization $D_{\alpha}$ of $\bar{\partial}_{J, H_{\alpha}}$ at $v_{o}$ with the above Lagrangian boundary conditions can be viewed as an operator $D_{\alpha}$ on

$$
W_{i \mathbb{R}^{n}, \mathbb{R}^{n}}^{1, p}(\alpha):=\{v:] 0,1\left[\times \mathbb{R} / \alpha^{-1} \mathbb{Z} \rightarrow \mathbb{C}^{n} \mid v(0, \cdot) \in i \mathbb{R}^{n}, v(1, \cdot) \in \mathbb{R}^{n}\right\},
$$

with norm $\|\cdot\|_{1, p ; \alpha}$. Assuming that $D_{\alpha}^{-1}$ is not uniformly bounded as $\alpha \rightarrow 0$ means that we would have $\alpha_{n} \rightarrow 0$ and $v_{n} \in W_{i \mathbb{R}^{n}, \mathbb{R}^{n}}^{1, p}\left(\alpha_{n}\right)$ with $\left\|v_{n}\right\|_{1, p ; \alpha_{n}}=1$ such that $\left\|D_{\alpha_{n}} v_{n}\right\|_{0, p ; \alpha_{n}} \rightarrow 0$. The limit operator to compare to is the standard $\bar{\partial}$-operator on maps

$$
v:[0,1] \times \mathbb{R} \rightarrow \mathbb{C}^{n}, \text { such that } v(0, t) \in i \mathbb{R}^{n}, v(1, t) \in \mathbb{R}^{n} \forall t \in \mathbb{R} .
$$

This comparison operator is clearly an isomorphism, so one easily shows that $D_{\alpha_{n}}$ has to be invertible for $\alpha_{n}$ small.

Let $\beta \in C^{\infty}(\mathbb{R},[0,1])$ be a cut-off function such that

$$
\beta(t)=\left\{\begin{array}{ll}
1, & t \leq 0, \\
0, & t \geq 1,
\end{array} \quad \beta^{\prime} \leq 0,\right.
$$


and set $\beta_{n}(t)=\beta\left(\alpha_{n} t-1\right) \cdot \beta\left(-\alpha_{n} t\right)$, hence

$$
\beta_{n \mid\left[0, \alpha_{n}^{-1}\right]} \equiv 1 \quad \text { and } \quad \operatorname{supp} \beta_{n} \subset\left[-\alpha_{n}^{-1}, 2 \alpha_{n}^{-1}\right] \text {. }
$$

We have

$$
\left\|v_{n}\right\|_{1, p ; \alpha_{n}} \leq\left\|\beta_{n} v_{n}\right\|_{1, p ; \mathbb{R}} \leq c_{1}\left\|v_{n}\right\|_{1, p ; \alpha_{n}} .
$$

Since the linearization $D_{\alpha}$ is of the form

$$
D_{\alpha} v=\partial_{s} v+i \partial_{t} v+\alpha A(s, t) v
$$

with some matrix $A(s, t)$, we observe that

$$
\left\|\bar{\partial}\left(\beta_{n} v_{n}\right)-D_{\alpha_{n}}\left(\beta_{n} v_{n}\right)\right\|_{0, p ; \mathbb{R}}=\alpha_{n}\left\|A \beta_{n} v_{n}\right\|_{0, p ; \mathbb{R}} \rightarrow 0 .
$$

Moreover,

$$
\begin{aligned}
\left\|D_{\alpha_{n}}\left(\beta_{n} v_{n}\right)\right\|_{0, p ; \mathbb{R}} & \leq\left\|i \beta_{n}^{\prime} v_{n}\right\|_{0, p ; \mathbb{R}}+\left\|\beta_{n} D_{\alpha_{n}} v_{n}\right\|_{0, p ; \mathbb{R}} \\
& \leq c_{2} \alpha_{n}\left\|v_{n}\right\|_{0, p ;\left[-\alpha_{n}^{-1}, 0\right] \cup\left[\alpha_{n}^{-1}, 2 \alpha_{n}^{-1}\right]}+3\left\|D_{\alpha_{n}} v_{n}\right\|_{0, p ; \alpha_{n}} \\
& \leq c_{2} \alpha_{n} 2\left\|v_{n}\right\|_{1, p ; \alpha_{n}}+3\left\|D_{\alpha_{n}} v_{n}\right\|_{0, p ; \alpha_{n}} \rightarrow 0 .
\end{aligned}
$$

Hence, we find a subsequence such that $\beta_{n_{k}} v_{n_{k}} \rightarrow v_{o} \in \operatorname{ker} \bar{\partial}=\{0\}$ which means that $\left\|v_{n_{k}}\right\|_{1, p ; \alpha_{n_{k}}} \rightarrow 0$ in contradiction to $\left\|v_{n}\right\|=1$.

Similarly, we see that the coherent orientation for the determinant of $D_{\alpha_{n}}$ equals that of $\bar{\partial}$ which is canonically 1 . This completes the proof of the proposition.

From the cobordisms $\mu_{P_{i}}^{\mathrm{Ev}}(\gamma, x), i=1,2,3$, we now obtain the chain homotopy between $M$ ev and Ev० $\Phi-L^{\Lambda}$. This concludes the proof of Theorem 4.9.

Comparison between i! and $I_{!}$In Section 2.2, we have shown that the homomorphism $i_{\text {! }}$ is induced by the chain map

$$
M \mathrm{i}_{!}: M_{j}\left(\mathbb{S}_{L}^{\Lambda}\right) \rightarrow M_{j-n}\left(\mathbb{S}_{L}^{\Omega}\right)
$$

between the Morse complexes of the Lagrangian action functional on the spaces $\Lambda^{1}(M)$

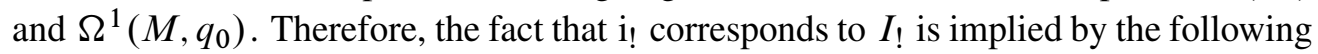
chain level result:

Theorem 4.11 The diagram

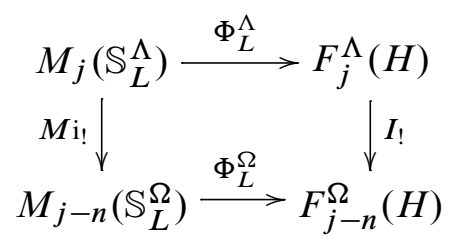

is chain homotopy commutative. 
Indeed, one can show that both $I_{!} \circ \Phi_{L}^{\Lambda}$ and $\Phi_{L}^{\Omega} \circ M$ i! are homotopic to the same chain map $K^{!}$. The definition of $K^{!}$makes use of the following spaces: Given $\gamma \in \mathscr{P}^{\Lambda}(L)$ and $x \in \mathscr{P}^{\Omega}(H)$, set

$\mu_{K}^{!}(\gamma, x):=\left\{u:\left[0,+\infty\left[\times[0,1] \rightarrow T^{*} M \mid \bar{\partial}_{J, H}(u)=0\right.\right.\right.$,

$\left.\pi \circ u(s, 0)=\pi \circ u(s, 1)=q_{0} \forall s \geq 0, \pi \circ u(0, \cdot) \in W^{u}\left(\gamma ; X_{L}^{\Lambda}\right), \lim _{s \rightarrow+\infty} u(s, \cdot)=x\right\}$.

Again, details are left to the reader.

We conclude that all the homomorphisms which appear in diagram (1-1) have their Floer homological counterpart: The vertical arrows have been treated in this section, whereas the horizontal ones are described in Appendix A.4 and Sections 4.3 and 4.2.

\section{Linear theory}

The remaining part of this paper consists of technical tools. In this section we develop the linear theory which allows us to study the Floer problems on the various Riemann surfaces introduced in the previous sections. All these elliptic problems are treated in the unifying setting of Cauchy-Riemann operators on strips with jumping nonlocal conormal boundary conditions.

\subsection{The Maslov index}

Let $\eta_{0}$ be the Liouville one-form on $T^{*} \mathbb{R}^{n}=\mathbb{R}^{n} \times\left(\mathbb{R}^{n}\right)^{*}$, that is the tautological one-form $\eta_{0}=p d q$, that is

$$
\eta_{0}(q, p)[(u, v)]:=p[u], \quad \text { for } q, u \in \mathbb{R}^{n}, p, v \in\left(\mathbb{R}^{n}\right)^{*} .
$$

Its differential $\omega_{0}=d \eta_{0}=d p \wedge d q$,

$$
\omega_{0}\left[\left(q_{1}, p_{1}\right),\left(q_{2}, p_{1}\right)\right]=p_{1}\left[q_{2}\right]-p_{2}\left[q_{1}\right], \quad \text { for } q_{1}, q_{2} \in \mathbb{R}^{n}, p_{1}, p_{2} \in\left(\mathbb{R}^{n}\right)^{*},
$$

is the standard symplectic form on $T^{*} \mathbb{R}^{n}$.

The symplectic group, that is the group of linear automorphisms of $T^{*} \mathbb{R}^{n}$ preserving $\omega_{0}$, is denoted by $\operatorname{Sp}(2 n)$. Let $\mathscr{L}(n)$ be the Grassmannian of Lagrangian subspaces of $T^{*} \mathbb{R}^{n}$, that is the set of $n$-dimensional linear subspaces of $T^{*} \mathbb{R}^{n}$ on which $\omega_{0}$ vanishes. The relative Maslov index assigns to every pair of Lagrangian paths $\lambda_{1}, \lambda_{2}:[a, b] \rightarrow \mathscr{L}(n)$ a half integer $\mu\left(\lambda_{1}, \lambda_{2}\right)$. We refer to [42] for the definition and for the properties of the relative Maslov index.

Another useful invariant is the Hörmander index of four Lagrangian subspaces (see Hörmander [33], Duistermaat [20] or Robbin and Salamon [42]): 
Definition 5.1 Let $\lambda_{0}, \lambda_{1}, v_{0}, v_{1}$ be four Lagrangian subspaces of $T^{*} \mathbb{R}^{n}$. Their Hörmander index is the half integer

$$
h\left(\lambda_{0}, \lambda_{1} ; v_{0}, v_{1}\right):=\mu\left(v, \lambda_{1}\right)-\mu\left(v, \lambda_{0}\right),
$$

where $v:[0,1] \rightarrow \mathscr{L}(n)$ is a Lagrangian path such that $v(0)=v_{0}$ and $v(1)=v_{1}$.

Indeed, the quantity defined above does not depend on the choice of the Lagrangian path $v$ joining $v_{0}$ and $v_{1}$.

If $V$ is a linear subspace of $\mathbb{R}^{n}, N^{*} V \subset T^{*} \mathbb{R}^{n}$ denotes its conormal space, that is

$$
N^{*} V:=\left\{(q, p) \in \mathbb{R}^{n} \times\left(\mathbb{R}^{n}\right)^{*} \mid q \in V, V \subset \operatorname{ker} p\right\}=V \times V^{\perp},
$$

where $V^{\perp}$ denotes the set of covectors in $\left(\mathbb{R}^{n}\right)^{*}$ which vanish on $V$. Conormal spaces are Lagrangian subspaces of $T^{*} \mathbb{R}^{n}$.

Let $C: T^{*} \mathbb{R}^{n} \rightarrow T^{*} \mathbb{R}^{n}$ be the linear involution

$$
C(q, p):=(q,-p) \quad \forall(q, p) \in T^{*} \mathbb{R}^{n} .
$$

The involution $C$ is antisymplectic, meaning that

$$
\omega_{0}(C \xi, C \eta)=-\omega_{0}(\xi, \eta) \quad \forall \xi, \eta \in T^{*} \mathbb{R}^{n} .
$$

In particular, $C$ maps Lagrangian subspaces into Lagrangian subspaces. Since the Maslov index is natural with respect to symplectic transformations and changes sign if we change the sign of the symplectic structure, we have the identity

$$
\mu(C \lambda, C \nu)=-\mu(\lambda, \nu),
$$

for every pair of Lagrangian paths $\lambda, v:[a, b] \rightarrow \mathscr{L}(n)$. Since conormal subspaces are $C$-invariant, we deduce that

$$
\mu\left(N^{*} V, N^{*} W\right)=0,
$$

for every pair of paths $V, W$ into the Grassmannian of $\mathbb{R}^{n}$. Let $V_{0}, V_{1}, W_{0}, W_{1}$ be four linear subspaces of $\mathbb{R}^{n}$, and let $v:[0,1] \rightarrow \mathscr{L}(n)$ be a Lagrangian path such that $v(0)=N^{*} W_{0}$ and $v(1)=N^{*} W_{1}$. By (5-1),

$$
\begin{aligned}
h\left(N^{*} V_{0}, N^{*} V_{1} ; N^{*} W_{0}, N^{*} W_{1}\right) & =\mu\left(\nu, N^{*} V_{1}\right)-\mu\left(\nu, N^{*} V_{0}\right) \\
& =-\mu\left(C \nu, N^{*} V_{1}\right)+\mu\left(C v, N^{*} V_{0}\right) .
\end{aligned}
$$

But also the Lagrangian path $C v$ joins $N^{*} W_{0}$ and $N^{*} W_{1}$, so the latter quantity equals

$$
-h\left(N^{*} V_{0}, N^{*} V_{1} ; N^{*} W_{0}, N^{*} W_{1}\right) .
$$

We deduce the following: 
Proposition 5.2 Let $V_{0}, V_{1}, W_{0}, W_{1}$ be four linear subspaces of $\mathbb{R}^{n}$. Then

$$
h\left(N^{*} V_{0}, N^{*} V_{1} ; N^{*} W_{0}, N^{*} W_{1}\right)=0 .
$$

We identify the product $T^{*} \mathbb{R}^{n} \times T^{*} \mathbb{R}^{n}$ with $T^{*} \mathbb{R}^{2 n}$, and we endow it with its standard symplectic structure. In other words, we consider the product symplectic form, not the twisted one used in [42]. Note that the conormal space of the diagonal $\Delta_{\mathbb{R}^{n}}$ in $\mathbb{R}^{n} \times \mathbb{R}^{n}$ is the graph of $C$ :

$$
N^{*} \Delta_{\mathbb{R}^{n}}=\operatorname{graph} C \subset T^{*} \mathbb{R}^{n} \times T^{*} \mathbb{R}^{n}=T^{*} \mathbb{R}^{2 n} .
$$

The linear endomorphism $\Psi$ of $T^{*} \mathbb{R}^{n}$ belongs to the symplectic group $\operatorname{Sp}(2 n)$ if and only if the graph of the linear endomorphism $\Psi C$ is a Lagrangian subspace of $T^{*} \mathbb{R}^{2 n}$, if and only if the graph of $C \Psi$ is a Lagrangian subspace of $T^{*} \mathbb{R}^{2 n}$. If $\lambda_{1}, \lambda_{2}$ are paths of Lagrangian subspaces of $T^{*} \mathbb{R}^{n}$ and $\Psi$ is a path in $\operatorname{Sp}(2 n)$, Theorem 3.2 of [42] leads to the identities

$$
\mu\left(\Psi \lambda_{1}, \lambda_{2}\right)=\mu\left(\operatorname{graph} \Psi C, C \lambda_{1} \times \lambda_{2}\right)=-\mu\left(\operatorname{graph} C \Psi, \lambda_{1} \times C \lambda_{2}\right) .
$$

The Conley-Zehnder index $\mu_{C Z}(\Psi)$ of a symplectic path $\Psi:[0,1] \rightarrow \operatorname{Sp}(2 n)$ is related to the relative Maslov index by the formula

$$
\mu_{C Z}(\Psi)=\mu\left(\operatorname{graph} \Psi C, N^{*} \Delta_{\mathbb{R}^{n}}\right)=\mu\left(N^{*} \Delta_{\mathbb{R}^{n}}, \operatorname{graph} C \Psi\right) .
$$

We conclude this section by fixing some standard identifications, which allow to see $T^{*} \mathbb{R}^{n}$ as a complex vector space. By using the Euclidean inner product on $\mathbb{R}^{n}$, we can identify $T^{*} \mathbb{R}^{n}$ with $\mathbb{R}^{2 n}$. We also identify the latter space to $\mathbb{C}^{n}$, by means of the isomorphism $(q, p) \mapsto q+i p$. In other words, we consider the complex structure

$$
J_{0}:=\left(\begin{array}{cc}
0 & -I \\
I & 0
\end{array}\right)
$$

on $\mathbb{R}^{2 n}$. With these identifications, the Euclidean inner product $u \cdot v$, respectively the symplectic product $\omega_{0}(u, v)$, of two vectors $u, v \in T^{*} \mathbb{R}^{n} \cong \mathbb{R}^{2 n} \cong \mathbb{C}^{n}$ is the real part, respectively the imaginary part, of their Hermitian product $\langle\cdot, \cdot\rangle$,

$$
\langle u, v\rangle:=\sum_{j=1}^{n} u_{j} \overline{v_{j}}=u \cdot v+i \omega_{0}(u, v) .
$$

The involution $C$ is the complex conjugation. By identifying $V^{\perp}$ with the Euclidean orthogonal complement, we have

$$
N^{*} V=V \oplus i V^{\perp}=\left\{z \in \mathbb{C}^{n} \mid \operatorname{Re} z \in V, \operatorname{Im} z \in V^{\perp}\right\} .
$$


If $\lambda:[0,1] \rightarrow \mathscr{L}(1)$ is the path

$$
\lambda(t)=e^{i \alpha t} \mathbb{R}, \quad \alpha \in \mathbb{R},
$$

the relative Maslov index of $\lambda$ with respect to $\mathbb{R}$ is the half integer

$$
\mu(\lambda, \mathbb{R})= \begin{cases}-1 / 2-\lfloor\alpha / \pi\rfloor & \text { if } \alpha \in \mathbb{R} \backslash \pi \mathbb{Z}, \\ -\alpha / \pi & \text { if } \alpha \in \pi \mathbb{Z} .\end{cases}
$$

Notice that the sign is different from the one appearing in [42] (localization axiom in Theorem 2.3), due to the fact that we are using the opposite symplectic form on $\mathbb{R}^{2 n}$. Our sign convention here also differs from the one used in [4], because we are using the opposite complex structure on $\mathbb{R}^{2 n}$.

\subsection{Elliptic estimates on the quadrant}

We recall that a real linear subspace $V$ of $\mathbb{C}^{n}$ is said to be totally real if $V \cap i V=(0)$. Denote by $\mathbb{H}$ the upper half-plane $\{z \in \mathbb{C} \mid \operatorname{Im} z>0\}$, and by $\mathbb{H}^{+}$the upper-right quadrant $\{z \in \mathbb{H} \mid \operatorname{Re} z>0\}$. We shall make use of the following Calderon-Zygmund estimates for the Cauchy-Riemann operator $\bar{\partial}=\partial_{s}+i \partial_{t}$ :

Theorem 5.3 Let $V$ be an $n$-dimensional totally real subspace of $\mathbb{C}^{n}$. For every $p \in] 1,+\infty[$, there exists a constant $c=c(p, n)$ such that

$$
\|D u\|_{L^{p}} \leq c\|\bar{\partial} u\|_{L^{p}}
$$

for every $u \in C_{c}^{\infty}\left(\mathbb{C}, \mathbb{C}^{n}\right)$, and for every $u \in C_{c}^{\infty}\left(\mathrm{Cl}(\mathbb{H}), \mathbb{C}^{n}\right)$ such that $u(s) \in V$ for every $s \in \mathbb{R}$.

We shall also need the following regularity result for weak solutions of $\bar{\partial}$. Denoting by $\partial:=\partial_{s}-i \partial_{t}$ the anti-Cauchy-Riemann operator, we have:

Theorem 5.4 (Regularity of weak solutions of $\bar{\partial}$ ) Let $V$ be an $n$-dimensional totally real subspace of $\mathbb{C}^{n}$, and let $1<p<\infty, k \in \mathbb{N}$.

(i) Let $u \in L_{\mathrm{loc}}^{p}\left(\mathbb{C}, \mathbb{C}^{n}\right), f \in W_{\mathrm{loc}}^{k, p}\left(\mathbb{C}, \mathbb{C}^{n}\right)$ be such that

$$
\operatorname{Re} \int_{\mathbb{C}}\langle u, \partial \varphi\rangle d s d t=-\operatorname{Re} \int_{\mathbb{C}}\langle f, \varphi\rangle d s d t
$$

for every $\varphi \in C_{c}^{\infty}\left(\mathbb{C}, \mathbb{C}^{n}\right)$. Then $u \in W_{\mathrm{loc}}^{k+1, p}\left(\mathbb{C}, \mathbb{C}^{n}\right)$ and $\bar{\partial} u=f$. 
(ii) Let $u \in L^{p}\left(\mathbb{H}, \mathbb{C}^{n}\right), f \in W^{k, p}\left(\mathbb{H}, \mathbb{C}^{n}\right)$ be such that

$$
\operatorname{Re} \int_{\mathbb{H}}\langle u, \partial \varphi\rangle d s d t=-\operatorname{Re} \int_{\mathbb{H}}\langle f, \varphi\rangle d s d t,
$$

for every $\varphi \in C_{c}^{\infty}\left(\mathbb{C}, \mathbb{C}^{n}\right)$ such that $\varphi(\mathbb{R}) \subset V$. Then $u \in W^{k+1, p}\left(\mathbb{H}, \mathbb{C}^{n}\right)$, $\bar{\partial} u=f$, and the trace of $u$ on $\mathbb{R}$ takes values into the $\omega_{0}$-orthogonal complement of $V$ :

$$
V^{\perp \omega_{0}}:=\left\{\xi \in \mathbb{C}^{n} \mid \omega_{0}(\xi, \eta)=0 \forall \eta \in V\right\}
$$

Remark 5.5 If we replace the upper half-plane $\mathbb{H}$ in (ii) by the right half-plane $\{\operatorname{Re} z>0\}$ and the test mappings $\varphi \in C_{c}^{\infty}\left(\mathbb{C}, \mathbb{C}^{n}\right)$ satisfy $\varphi(i \mathbb{R}) \subset V$, then the trace of $u$ on $i \mathbb{R}$ takes value into $V^{\perp}$, the Euclidean orthogonal complement of $V$ in $\mathbb{R}^{2 n}$.

Two linear subspaces $V, W$ of $\mathbb{R}^{n}$ are said to be partially orthogonal if the linear subspaces $V \cap(V \cap W)^{\perp}$ and $W \cap(V \cap W)^{\perp}$ are orthogonal, that is if their projections into the quotient $\mathbb{R}^{n} / V \cap W$ are orthogonal.

Lemma 5.6 Let $V$ and $W$ be partially orthogonal linear subspaces of $\mathbb{R}^{n}$. For every $p \in] 1,+\infty[$, there exists a constant $c=c(p, n)$ such that

$$
\|D u\|_{L^{p}} \leq c\|\bar{\partial} u\|_{L^{p}}
$$

for every $u \in C_{c}^{\infty}\left(\mathrm{Cl}\left(\mathbb{H}^{+}\right), \mathbb{C}^{n}\right)$ such that

$$
u(s) \in N^{*} V, \quad u(i s) \in N^{*} W, \quad \forall t \in[0,+\infty[.
$$

Proof Since $V$ and $W$ are partially orthogonal, $\mathbb{R}^{n}$ has an orthogonal splitting $\mathbb{R}^{n}=X_{1} \oplus X_{2} \oplus X_{3} \oplus X_{4}$ such that

$$
V=X_{1} \oplus X_{2}, \quad W=X_{1} \oplus X_{3} .
$$

Therefore,

$$
N^{*} V=X_{1} \oplus X_{2} \oplus i X_{3} \oplus i X_{4}, \quad N^{*} W=X_{1} \oplus X_{3} \oplus i X_{2} \oplus i X_{4} .
$$

Let $U \in \mathrm{U}(n)$ be the identity on $\left(X_{1} \oplus X_{2}\right) \otimes \mathbb{C}$, and the multiplication by $i$ on $\left(X_{3} \oplus X_{4}\right) \otimes \mathbb{C}$. Then

$$
U N^{*} V=\mathbb{R}^{n}, \quad U N^{*} W=X_{1} \oplus X_{4} \oplus i X_{2} \oplus i X_{3}=N^{*}\left(X_{1} \oplus X_{4}\right) .
$$

Up to multiplying $u$ by $U$, we can replace the boundary conditions (5-7) by

$$
u(s) \in \mathbb{R}^{n} \quad u(i s) \in Y, \quad \forall s \in[0,+\infty[,
$$


where $Y$ is a totally real $n$-dimensional subspace of $\mathbb{C}^{n}$ such that $\bar{Y}=Y$. Define a $\mathbb{C}^{n}$-valued map $v$ on the right half-plane $\{\operatorname{Re} z \geq 0\}$ by Schwarz reflection:

$$
v(z):= \begin{cases}u(z) & \text { if } \operatorname{Im} z \geq 0 \\ \overline{u(\bar{z})} & \text { if } \operatorname{Im} z \leq 0\end{cases}
$$

By (5-8) and by the fact that $Y$ is self-conjugate, $v$ belongs to $W^{1, p}\left(\{\operatorname{Re} z>0\}, \mathbb{C}^{n}\right)$, and satisfies

$$
v(i t) \in Y \quad \forall t \in \mathbb{R}
$$

Moreover,

$$
\|\nabla v\|_{L^{p}(\{\operatorname{Re} z>0\})}^{p}=2\|\nabla v\|_{L^{p}(\mathbb{H}+)}^{p},
$$

and since $\bar{\partial} v(z)=\overline{\bar{\partial}} u(\bar{z})$ for $\operatorname{Im} z \leq 0$,

$$
\|\bar{\partial} v\|_{L^{p}(\{\operatorname{Re} z>0\})}^{p}=2\|\bar{\partial} v\|_{L^{p}\left(\mathbb{H}^{+}\right)}^{p} .
$$

Then (5-6) follows from the Calderon-Zygmund estimate on the half plane with totally real boundary conditions (Theorem 5.3).

Similarly, Theorem 5.4 has the following consequence about regularity of weak solutions of $\bar{\partial}$ on the upper right quadrant $\mathbb{H}^{+}$:

Lemma 5.7 Let $V$ and $W$ be partially orthogonal linear subspaces of $\mathbb{R}^{n}$. Let $u \in L^{p}\left(\mathbb{H}^{+}, \mathbb{C}^{n}\right), f \in L^{p}\left(\mathbb{H}^{+}, \mathbb{C}^{n}\right), 1<p<\infty$, be such that

$$
\operatorname{Re} \int_{\mathbb{H}^{+}}\langle u, \partial \varphi\rangle d s d t=-\operatorname{Re} \int_{\mathbb{H}^{+}}\langle f, \varphi\rangle d s d t,
$$

for every $\varphi \in C_{c}^{\infty}\left(\mathbb{C}, \mathbb{C}^{n}\right)$ such that $\varphi(\mathbb{R}) \subset N^{*} V, \varphi(i \mathbb{R}) \subset N^{*} W$. Then $u \in$ $W^{1, p}\left(\mathbb{H}^{+}, \mathbb{C}^{n}\right), \bar{\partial} u=f$, the trace of $u$ on $\mathbb{R}$ takes values into $N^{*} V$, and the trace of $u$ on $i \mathbb{R}$ takes values into $\left(N^{*} W\right)^{\perp}=N^{*}\left(W^{\perp}\right)=i N^{*} W$.

Proof By means of a linear unitary transformation, as in the proof of Lemma 5.6, we may assume that $V=N^{*} V=\mathbb{R}^{n}$. A Schwarz reflection then allows to extend $u$ to a map $v$ on the right half-plane $\{\operatorname{Re} z>0\}$ which is in $L^{p}$ and is a weak solution of $\bar{\partial} v=g \in L^{p}$, with boundary condition in $i N^{*} W$ on $i \mathbb{R}$. The thesis follows from Theorem 5.4 .

We are now interested in studying the operator $\bar{\partial}$ on the half-plane $\mathbb{H}$, with boundary conditions

$$
u(s) \in N^{*} V, \quad u(-s) \in N^{*} W \quad \forall s>0,
$$


where $V$ and $W$ are partially orthogonal linear subspaces of $\mathbb{R}^{n}$. Taking Lemma 5.6 and Lemma 5.7 into account, the natural idea is to obtain the required estimates by applying a conformal change of variable mapping the half-plane $\mathbb{H}$ onto the upper right quadrant $\mathbb{H}^{+}$. More precisely, let $\mathscr{R}$ and $\mathscr{T}$ be the transformations

$$
\begin{array}{ll}
\mathscr{R}: \operatorname{Map}\left(\mathbb{H}, \mathbb{C}^{n}\right) \rightarrow \operatorname{Map}\left(\mathbb{H}^{+}, \mathbb{C}^{n}\right), & (\mathscr{T} u)(\zeta)=u\left(\zeta^{2}\right), \\
\mathscr{T}: \operatorname{Map}\left(\mathbb{H}, \mathbb{C}^{n}\right) \rightarrow \operatorname{Map}\left(\mathbb{H}^{+}, \mathbb{C}^{n}\right), & (\mathscr{T} u)(\zeta)=2 \bar{\zeta} u\left(\zeta^{2}\right),
\end{array}
$$

where Map denotes some space of maps. Then the diagram

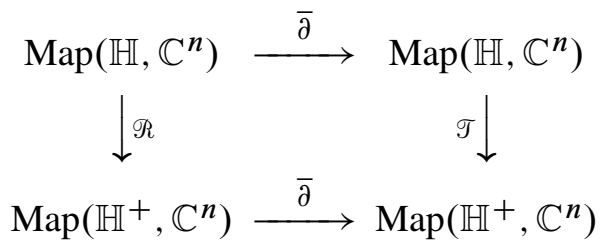

commutes. By the elliptic estimates of Lemma 5.6, suitable domain and codomain for the operator on the lower horizontal arrow are the standard $W^{1, p}$ and $L^{p}$ spaces, for $1<p<\infty$. Moreover, if $u \in \operatorname{Map}\left(\mathbb{H}, \mathbb{C}^{n}\right)$ then

$$
\begin{aligned}
\|\mathscr{R} u\|_{L^{p}\left(\mathbb{H}^{+}\right)}^{p} & =\frac{1}{4} \int_{\mathbb{H}} \frac{1}{|z|}|u(z)|^{p} d s d t, \\
\|D(\mathscr{R} u)\|_{L^{p}\left(\mathbb{H}^{+}\right)}^{p} & =2^{p-2} \int_{\mathbb{H}}|D u(z)|^{p}|z|^{p / 2-1} d s d t, \\
\|\mathscr{T} u\|_{L^{p}\left(\mathbb{H}^{+}\right)}^{p} & =2^{p-2} \int_{\mathbb{H}}|u(z)|^{p}|z|^{p / 2-1} d s d t .
\end{aligned}
$$

Note also that by the generalized Poincaré inequality, the $W^{1, p}$ norm on $\mathbb{H}^{+} \cap \mathbb{D}_{r}$, where $\mathbb{D}_{r}$ denotes the open disk of radius $r$, is equivalent to the norm

$$
\|v\|_{\widetilde{W}^{1, p}\left(\mathbb{H}^{+} \cap \mathbb{D}_{r}\right)}^{p}:=\|D v\|_{L^{p}\left(\mathbb{H}^{+} \cap \mathbb{D}_{r}\right)}^{p}+\int_{\mathbb{H}^{+} \cap \mathbb{D}_{r}}|v(\zeta)|^{p}|\zeta|^{p} d \sigma d \tau,
$$

and the $\widetilde{W}^{1, p}$ norm of $\mathscr{R} u$ is

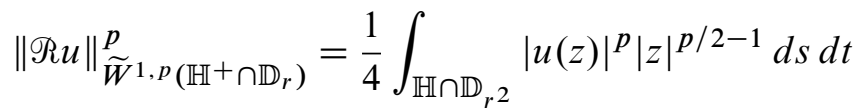

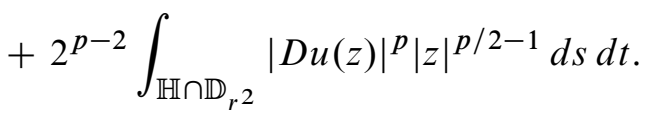

So when dealing with bounded domains, both the transformations $\mathscr{R}$ and $\mathscr{T}$ involve the appearance of the weight $|z|^{p / 2-1}$ in the $L^{p}$ norms. Note also that when $p=2$, this 
weight is just 1 , reflecting the fact that the $L^{2}$ norm of the differential is a conformal invariant.

By the commutativity of diagram (5-11) and by the identities (5-13), (5-14), Lemma 5.6 applied to $\mathscr{R} u$ implies the following:

Lemma 5.8 Let $V$ and $W$ be partially orthogonal linear subspaces of $\mathbb{R}^{n}$. For every $p \in] 1,+\infty[$, there exists a constant $c=c(p, n)$ such that

$$
\int_{\mathbb{H}}|\nabla u(z)|^{p}|z|^{p / 2-1} d s d t \leq c^{p} \int_{\mathbb{H}}|\bar{\partial} u(z)|^{p}|z|^{p / 2-1} d s d t
$$

for every compactly supported map $u: \mathrm{Cl}(\mathbb{H}) \rightarrow \mathbb{C}^{n}$ such that $\zeta \mapsto u\left(\zeta^{2}\right)$ is smooth on $\mathrm{Cl}\left(\mathbb{H}^{+}\right)$, and

$$
u(-s) \in N^{*} V, \quad u(s) \in N^{*} W, \quad \forall s \in[0,+\infty[.
$$

\subsection{Strips with jumping conormal boundary conditions}

Let us consider the following data: two integers $k, k^{\prime} \geq 0, k+1$ linear subspaces $V_{0}, \ldots, V_{k}$ of $\mathbb{R}^{n}$ such that $V_{j-1}$ and $V_{j}$ are partially orthogonal, for every $j=$ $1, \ldots, k, k^{\prime}+1$ linear subspaces $V_{0}^{\prime}, \ldots, V_{k}^{\prime}$ of $\mathbb{R}^{n}$ such that $V_{j-1}^{\prime}$ and $V_{j}^{\prime}$ are partially orthogonal, for every $j=1, \ldots, k^{\prime}$, and real numbers

$$
\begin{aligned}
& -\infty=s_{0}<s_{1}<\cdots<s_{k}<s_{k+1}=+\infty, \\
& -\infty=s_{0}^{\prime}<s_{1}^{\prime}<\cdots<s_{k^{\prime}}^{\prime}<s_{k^{\prime}+1}^{\prime}=+\infty .
\end{aligned}
$$

Denote by $\mathscr{V}$ the $(k+1)$-tuple $\left(V_{0}, \ldots, V_{k}\right)$, by $\mathscr{V}^{\prime}$ the $\left(k^{\prime}+1\right)$-tuple $\left(V_{0}^{\prime}, \ldots, V_{k}^{\prime}\right)$, and set

$$
\mathscr{S}:=\left\{s_{1}, \ldots, s_{k}, s_{1}^{\prime}+i, \ldots, s_{k^{\prime}}^{\prime}+i\right\} .
$$

Let $\Sigma$ be the closed strip

$$
\Sigma:=\{z \in \mathbb{C} \mid 0 \leq \operatorname{Im} z \leq 1\} .
$$

The space $C_{\mathscr{S}}^{\infty}\left(\Sigma, \mathbb{C}^{n}\right)$ is the space of maps $u: \Sigma \rightarrow \mathbb{C}^{n}$ which are smooth on $\Sigma \backslash \mathscr{Y}$, and such that the maps $\zeta \mapsto u\left(s_{j}+\zeta^{2}\right)$ and $\zeta \mapsto u\left(s_{j}^{\prime}+i-\zeta^{2}\right)$ are smooth in a neighborhood of 0 in the closed upper-right quadrant

$$
\mathrm{Cl}\left(\mathbb{H}^{+}\right)=\{\zeta \in \mathbb{C} \mid \operatorname{Re} \zeta \geq 0, \operatorname{Im} \zeta \geq 0\} .
$$

The symbol $C_{\mathscr{Y}, c}^{\infty}$ indicates bounded support.

Given $p \in\left[1,+\infty\left[\right.\right.$, we define the $X^{p}$ norm of a map $u \in L_{\text {loc }}^{1}\left(\Sigma, \mathbb{C}^{n}\right)$ by

$$
\|u\|_{X^{p}(\Sigma)}^{p}:=\|u\|_{L^{p}\left(\Sigma \backslash B_{r}(\varphi)\right)}^{p}+\sum_{w \in \Psi^{\prime}} \int_{\Sigma \cap B_{r}(w)}|u(z)|^{p}|z-w|^{p / 2-1} d s d t,
$$


where $r<1$ is less than half of the minimal distance between pairs of distinct points in $\mathscr{S}$. This is just a weighted $L^{p}$ norm, where the weight $|z-w|^{p / 2-1}$ comes from the identities (5-13), (5-14), and (5-16) of the last section. Note that when $p>2$ the $X^{p}$ norm is weaker than the $L^{p}$ norm, when $p<2$ the $X^{p}$ norm is stronger than the $L^{p}$ norm, and when $p=2$ the two norms are equivalent.

The space $X_{\varphi}^{p}\left(\Sigma, \mathbb{C}^{n}\right)$ is the space of locally integrable $\mathbb{C}^{n}$-valued maps on $\Sigma$ whose $X^{p}$ norm is finite. The $X^{p}$ norm makes it a Banach space. We view it as a real Banach space.

The space $X_{\mathscr{S}}^{1, p}\left(\Sigma, \mathbb{C}^{n}\right)$ is defined as the completion of the space $C_{\mathscr{S}, c}^{\infty}\left(\Sigma, \mathbb{C}^{n}\right)$ with respect to the norm

$$
\|u\|_{X^{1, p}(\Sigma)}^{p}:=\|u\|_{X^{p}(\Sigma)}^{p}+\|D u\|_{X^{p}(\Sigma)}^{p} .
$$

It is a Banach space with the above norm. Equivalently, it is the space of maps in $X^{p}\left(\Sigma, \mathbb{C}^{n}\right)$ whose distributional derivative is also in $X^{p}$. The space $X_{\mathscr{Y}, q, \mathscr{V}^{\prime}}^{1, p}\left(\Sigma, \mathbb{C}^{n}\right)$ is defined as the closure in $X_{\mathscr{S}}^{1, p}\left(\Sigma, \mathbb{C}^{n}\right)$ of the space of all $u \in C_{\mathscr{S}, c}^{\infty}\left(\Sigma, \mathbb{C}^{n}\right)$ such that

$$
\begin{array}{ll}
u(s) \in N^{*} V_{j} & \forall s \in\left[s_{j}, s_{j+1}\right], j=0, \ldots, k, \\
u(s+i) \in N^{*} V_{j}^{\prime} & \forall s \in\left[s_{j}^{\prime}, s_{j+1}^{\prime}\right], j=0, \ldots, k^{\prime} .
\end{array}
$$

Equivalently, it can be defined in terms of the trace of $u$ on the boundary of $\Sigma$.

Let $A: \mathbb{R} \times[0,1] \rightarrow \mathrm{L}\left(\mathbb{R}^{2 n}, \mathbb{R}^{2 n}\right)$ be continuous and bounded. For every $p \in[1,+\infty[$, the linear operator

$$
\bar{\partial}_{A}: X_{\varphi}^{1, p}\left(\Sigma, \mathbb{C}^{n}\right) \rightarrow X_{\varphi}^{p}\left(\Sigma, \mathbb{C}^{n}\right), \quad \bar{\partial}_{A} u:=\bar{\partial} u+A u,
$$

is bounded. Indeed, $\bar{\partial}$ is a bounded operator because of the inequality $|\bar{\partial} u| \leq|D u|$, while the multiplication operator by $A$ is bounded because

$$
\|A u\|_{X^{p}(\Sigma)} \leq\|A\|_{\infty}\|u\|_{X^{p}(\Sigma)} .
$$

We wish to prove that if $p>1$ and $A(z)$ satisfies suitable asymptotics for $\operatorname{Re} z \rightarrow \pm \infty$ the operator $\bar{\partial}_{A}$ restricted to the space $X_{\mathscr{Y}, \mathcal{V}, \mathscr{V}^{\prime}}^{1, p}\left(\Sigma, \mathbb{C}^{n}\right)$ of maps satisfying the boundary conditions (5-17) is Fredholm.

Assume that $A \in C^{0}\left(\overline{\mathbb{R}} \times[0,1], \mathrm{L}\left(\mathbb{R}^{2 n}, \mathbb{R}^{2 n}\right)\right)$ is such that $A( \pm \infty, t) \in \operatorname{Sym}(2 n, \mathbb{R})$ for every $t \in[0,1]$. Define $\Phi^{+}, \Phi^{-}:[0,1] \rightarrow \operatorname{Sp}(2 n)$ to be the solutions of the linear Hamiltonian systems

$$
\frac{d}{d t} \Phi^{ \pm}(t)=i A( \pm \infty, t) \Phi^{ \pm}(t), \quad \Phi^{ \pm}(0)=I .
$$

Then we have the following: 
Theorem 5.9 Assume that $\Phi^{-}(1) N^{*} V_{0} \cap N^{*} V_{0}^{\prime}=(0)$ and $\Phi^{+}(1) N^{*} V_{k} \cap N^{*} V_{k^{\prime}}^{\prime}=$ (0). Then the bounded $\mathbb{R}$-linear operator

$$
\bar{\partial}_{A}: X_{\mathscr{S}, \mathscr{\gamma}, \mathscr{V}^{\prime}}^{1, p}\left(\Sigma, \mathbb{C}^{n}\right) \rightarrow X_{\mathscr{S}}^{p}\left(\Sigma, \mathbb{C}^{n}\right), \quad \bar{\partial}_{A} u=\bar{\partial} u+A u,
$$

is Fredholm of index

$$
\text { ind } \begin{aligned}
\bar{\partial}_{A}= & \mu\left(\Phi^{-} N^{*} V_{0}, N^{*} V_{0}^{\prime}\right)-\mu\left(\Phi^{+} N^{*} V_{k}, N^{*} V_{k^{\prime}}^{\prime}\right) \\
& -\frac{1}{2} \sum_{j=1}^{k}\left(\operatorname{dim} V_{j-1}+\operatorname{dim} V_{j}-2 \operatorname{dim} V_{j-1} \cap V_{j}\right) \\
& -\frac{1}{2} \sum_{j=1}^{k^{\prime}}\left(\operatorname{dim} V_{j-1}^{\prime}+\operatorname{dim} V_{j}^{\prime}-2 \operatorname{dim} V_{j-1}^{\prime} \cap V_{j}^{\prime}\right) .
\end{aligned}
$$

The proof of the Fredholm property for Cauchy-Riemann type operators is based on local estimates. By a partition of unity argument, the proof that $\bar{\partial}_{A}$ is Fredholm reduces to the Calderon-Zygmund estimates of Lemmas 5.6, 5.8, and to the invertibility of $\bar{\partial}_{A}$ when $A$ does not depend on $\operatorname{Re} z$ and there are no jumps in the boundary conditions. Details are contained in the next section. The index computation instead is based on homotopy arguments together with a Liouville type result stating that in a particular case with one jump the operator $\bar{\partial}_{A}$ is an isomorphism.

\subsection{The Fredholm property}

The elliptic estimates of Section 5.2 have the following consequence:

Lemma 5.10 For every $p \in] 1,+\infty\left[\right.$, there exist constants $c_{0}=c_{0}(p, n, \mathscr{Y})$ and $c_{1}=c_{1}\left(p, n, k+k^{\prime}\right)$ such that

$$
\|D u\|_{X^{p}} \leq c_{0}\|u\|_{X^{p}}+c_{1}\|\bar{\partial} u\|_{X^{p}},
$$

for every $u \in C_{\mathscr{Y}_{, c}}^{\infty}\left(\Sigma, \mathbb{C}^{n}\right)$ such that

$$
u(s) \in N^{*} V_{j} \quad \forall s \in\left[s_{j-1}, s_{j}\right], \quad u(s+i) \in N^{*} V_{j}^{\prime} \quad \forall s \in\left[s_{j-1}^{\prime}, s_{j}^{\prime}\right]
$$

for every $j$.

Proof Let $\left\{\psi_{1}, \psi_{2}\right\} \cup\left\{\varphi_{j}\right\}_{j=1}^{k+k^{\prime}}$ be a smooth partition of unity on $\mathbb{C}$ satisfying

$$
\begin{array}{rlrl}
\operatorname{supp} \psi_{1} & \subset\{z \in \mathbb{C} \mid \operatorname{Im} z<2 / 3\} \backslash B_{r / 2}(\mathscr{S}), & & \\
\operatorname{supp} \psi_{2} & \subset\{z \in \mathbb{C} \mid \operatorname{Im} z>1 / 3\} \backslash B_{r / 2}(\mathscr{S}), & \\
\operatorname{supp} \varphi_{j} & \subset B_{r}\left(s_{j}\right) & & \forall j=1, \ldots, k, \\
\operatorname{supp} \varphi_{k+j} & \subset B_{r}\left(s_{j}^{\prime}+i\right) & & \forall j=1, \ldots, k^{\prime} .
\end{array}
$$


By Lemma 5.8,

$$
\begin{aligned}
\left\|D\left(\varphi_{j} u\right)\right\|_{X^{p}(\Sigma)} & \leq c(p, n)\left\|\bar{\partial}\left(\varphi_{j} u\right)\right\|_{X^{p}(\Sigma)} \\
& \leq c(p, n)\left(\left\|\bar{\partial} \varphi_{j}\right\|_{\infty}\|u\|_{X^{p}(\Sigma)}+\|\bar{\partial} u\|_{X^{p}(\Sigma)}\right), \quad 1 \leq j \leq k+k^{\prime} .
\end{aligned}
$$

Since the $X^{p}$ norm is equivalent to the $L^{p}$ norm on the subspace of maps whose support does not meet $B_{r / 2}(\mathscr{S})$, the standard Calderon-Zygmund estimates on the half-plane (see Theorem 5.3) imply

$$
\begin{aligned}
\left\|D\left(\psi_{j} u\right)\right\|_{X^{p}(\Sigma)} & \leq c(p, n)\left\|\bar{\partial}\left(\psi_{j} u\right)\right\|_{X^{p}(\Sigma)} \\
& \leq c(p, n)\left(\left\|\bar{\partial} \psi_{j}\right\|_{\infty}\|u\|_{X^{p}(\Sigma)}+\|\bar{\partial} u\|_{X^{p}(\Sigma)}\right), \quad \forall j=1,2 .
\end{aligned}
$$

We conclude that

$$
\begin{aligned}
\|D u\|_{X^{p}} & \leq\left\|D\left(\psi_{1} u\right)\right\|_{X^{p}(\Sigma)}+\left\|D\left(\psi_{2} u\right)\right\|_{X^{p}(\Sigma)}+\sum_{j=1}^{k+k^{\prime}}\left\|D\left(\varphi_{j} u\right)\right\|_{X^{p}(\Sigma)} \\
& \leq c_{0}\|u\|_{X^{p}(\Sigma)}+c_{1}\|\bar{\partial} u\|_{X^{p}(\Sigma)},
\end{aligned}
$$

with

$c_{0}:=c(p, n)\left(\left\|\bar{\partial} \psi_{1}\right\|_{\infty}+\left\|\bar{\partial} \psi_{2}\right\|_{\infty}+\sum_{j=0}^{k+k^{\prime}+1}\left\|\bar{\partial} \varphi_{j}\right\|_{\infty}\right), \quad c_{1}:=\left(k+k^{\prime}+2\right) c(p, n)$,

as claimed.

The next result we need is the following theorem, proved in [43, Theorem 7.1]. Consider two continuously differentiable Lagrangian paths $\lambda, v: \overline{\mathbb{R}} \rightarrow \mathscr{L}(n)$, assumed to be constant on $\left[-\infty,-s_{0}\right]$ and on $\left[s_{0},+\infty\right]$, for some $s_{0}>0$. Denote by $W_{\lambda, v}^{1, p}\left(\Sigma, \mathbb{C}^{n}\right)$ the space of maps $u \in W^{1, p}\left(\Sigma, \mathbb{C}^{n}\right)$ such that $u(s, 0) \in \lambda(s)$ and $u(s, 1) \in v(s)$, for every $s \in \mathbb{R}$ (in the sense of traces). Let $A \in C^{0}\left(\overline{\mathbb{R}} \times[0,1], \mathrm{L}\left(\mathbb{R}^{2 n}, \mathbb{R}^{2 n}\right)\right)$ be such that $A( \pm \infty, t) \in \operatorname{Sym}(2 n, \mathbb{R})$ for any $t \in[0,1]$, and define $\Phi^{-}, \Phi^{+}:[0,1] \rightarrow \operatorname{Sp}(2 n)$ by $(5-18)$.

Theorem 5.11 (Cauchy-Riemann operators on the strip) Let $p \in] 1,+\infty[$, and assume that

$$
\Phi^{-}(1) \lambda(-\infty) \cap v(-\infty)=(0), \quad \Phi^{+}(1) \lambda(+\infty) \cap v(+\infty)=(0) .
$$

(i) The bounded $\mathbb{R}$-linear operator

$$
\bar{\partial}_{A}: W_{\lambda, v}^{1, p}\left(\Sigma, \mathbb{C}^{n}\right) \rightarrow L^{p}\left(\Sigma, \mathbb{C}^{n}\right), \quad \bar{\partial}_{A} u=\bar{\partial} u+A u,
$$


is Fredholm of index

$$
\text { ind } \bar{\partial}_{A}=\mu\left(\Phi^{-} \lambda(-\infty), v(-\infty)\right)-\mu\left(\Phi^{+} \lambda(+\infty), v(+\infty)\right)+\mu(\lambda, v) \text {. }
$$

(ii) If furthermore $A(s, t)=A(t), \lambda(s)=\lambda$, and $v(s)=v$ do not depend on $s$, the operator $\bar{\partial}_{A}$ is an isomorphism.

Note that under the assumptions of (ii) above, the equation $\bar{\partial} u+A u$ can be rewritten as $\partial_{s} u=-L_{A} u$, where $L_{A}$ is the unbounded $\mathbb{R}$-linear operator on $L^{2}\left([0,1], \mathbb{C}^{n}\right)$ defined by

$$
\begin{aligned}
\operatorname{dom} L_{A} & =W_{\lambda, v}^{1,2}\left([0,1], \mathbb{C}^{n}\right)=\left\{u \in W^{1,2}\left([0,1], \mathbb{C}^{n}\right) \mid u(0) \in \lambda, u(1) \in v\right\}, \\
L_{A} & =i \frac{d}{d t}+A .
\end{aligned}
$$

The conditions on $A$ imply that $L_{A}$ is self-adjoint and invertible. These facts lead to the following:

Proposition 5.12 Assume that $A, \lambda$, and $v$ satisfy the conditions of Theorem 5.11 (ii), and set $\delta:=\min \sigma\left(L_{A}\right) \cap\left[0,+\infty\left[>0\right.\right.$. Then for every $k \in \mathbb{N}$ there exists $c_{k}$ such that

$$
\|u(s, \cdot)\|_{C^{k}([0,1])} \leq c_{k}\|u(0, \cdot)\|_{L^{2}([0,1])} e^{-\delta s}, \quad \forall s \geq 0,
$$

for every $u \in W^{1, p}(] 0,+\infty[\times] 0,1\left[, \mathbb{C}^{n}\right), p>1$, such that $u(s, 0) \in \lambda, u(s, 1) \in v$ for every $s \geq 0$, and $\bar{\partial} u+A u=0$.

Next we need the following easy consequence of the Sobolev embedding theorem:

Proposition 5.13 Let $s>0$ and let $\chi_{s}$ be the characteristic function of the set $\{z \in \Sigma|| \operatorname{Re} z \mid \leq s\}$. Then the linear operator

$$
X_{\mathscr{S}}^{1, p}\left(\Sigma, \mathbb{C}^{n}\right) \rightarrow X_{\mathscr{Y}}^{q}\left(\Sigma, \mathbb{C}^{n}\right), \quad u \mapsto \chi_{s} u,
$$

is compact for every $q<\infty$ if $p \geq 2$, and for every $q<2 p /(2-p)$ if $1 \leq p<2$.

Proof Let $\left(u_{h}\right)$ be a bounded sequence in $X_{\mathscr{g}}^{1, p}\left(\Sigma, \mathbb{C}^{n}\right)$. Let $\left\{\psi_{1}, \psi_{2}\right\} \cup\left\{\varphi_{j}\right\}_{j=1}^{k+k^{\prime}}$ be a smooth partition of unity of $\mathbb{C}$ satisfying (5-20). Then the sequences $\left(\psi_{1} u_{h}\right)$, $\left(\psi_{2} u_{h}\right)$ and $\left(\varphi_{j} u_{h}\right)$, for $1 \leq j \leq k+k^{\prime}$ are bounded in $X^{1, p}\left(\Sigma, \mathbb{C}^{n}\right)$. We must show that each of these sequences is compact in $X_{\mathscr{G}}^{q}\left(\Sigma, \mathbb{C}^{n}\right)$.

Since the $X^{q}$ and $X^{1, p}$ norms on the space of maps supported in $\Sigma \backslash B_{r / 2}(\mathscr{S})$ are equivalent to the $L^{q}$ and $W^{1, p}$ norms, the Sobolev embedding theorem implies that the sequences $\left(\chi_{s} \psi_{1} u_{h}\right)$ and $\left(\chi_{s} \psi_{2} u_{h}\right)$ are compact in $X_{\mathscr{S}}^{q}\left(\Sigma, \mathbb{C}^{n}\right)$. 
Let $1 \leq j \leq k$. If $u$ is supported in $B_{r}\left(s_{j}\right)$, set $v(z):=u\left(s_{j}+z\right)$, so that by (5-12)

$$
\begin{aligned}
\|u\|_{X^{q}(\Sigma)}^{q}=\int_{B_{r}\left(s_{j}\right) \cap \Sigma}|u(z)|^{q}|z|^{q / 2-1} d s d t & \leq \int_{B_{r}\left(s_{j}\right) \cap \Sigma} \frac{1}{|z|}|u(z)|^{q} d s d t \\
& =4\|\Re u\|_{L^{q}(\mathbb{H}+\cap \mathbb{D} \sqrt{r})}^{q} .
\end{aligned}
$$

Set $v_{h}(z):=\varphi_{j}\left(s_{j}+z\right) u_{h}\left(s_{j}+z\right)$. By (5-16), the sequence $\left(\mathscr{R} v_{h}\right)$ is bounded in $W^{1, p}\left(\mathbb{H}^{+} \cap \mathbb{D} \sqrt{r}\right)$, hence it is compact in $L^{q}\left(\mathbb{H}^{+} \cap \mathbb{D} \sqrt{r}\right)$ for every $q<\infty$ if $p \geq 2$, and for every $q<2 p /(2-p)$ if $1 \leq p<2$. Then (5-21) implies that $\left(\varphi_{j} u_{h}\right)$ is compact in $X_{\varphi}^{q}\left(\Sigma, \mathbb{C}^{n}\right)$. A fortiori, so is $\left(\chi_{s} \varphi_{j} u_{h}\right)$. The same argument applies to $j \geq k+1$, concluding the proof.

Putting together Lemma 5.10, statement (ii) in Theorem 5.11, and the Proposition above we obtain the following:

Proposition 5.14 Let $1<p<\infty$. Assume that the paths of symmetric matrices $A( \pm \infty, \cdot)$ satisfy the assumptions of Theorem 5.9. Then

$$
\bar{\partial}_{A}: X_{\mathscr{S}, \mathscr{V}, \mathscr{V}^{\prime}}^{1, p}\left(\Sigma, \mathbb{C}^{n}\right) \rightarrow X_{\mathscr{\varphi}}^{p}\left(\Sigma, \mathbb{C}^{n}\right)
$$

is semi-Fredholm with ind $\bar{\partial}_{A}:=\operatorname{dim} \operatorname{ker} \bar{\partial}_{A}-\operatorname{dim} \operatorname{coker} \bar{\partial}_{A}<+\infty$.

Proof We claim that there exist $c \geq 0$ and $s \geq 0$ such that, for any $u \in X_{\mathscr{Y}, \mathscr{V}, \mathscr{V}^{\prime}}^{1, p}\left(\Sigma, \mathbb{C}^{n}\right)$, there holds

$$
\|u\|_{X^{1, p}(\Sigma)} \leq c\left(\|(\bar{\partial}+A) u\|_{X^{p}(\Sigma)}+\left\|\chi_{s} u\right\|_{X^{p}(\Sigma)}\right),
$$

where $\chi_{s}$ is the characteristic function of the set $\{z \in \Sigma|| \operatorname{Re} z \mid \leq s\}$.

By Theorem 5.11 (ii), the asymptotic operators

$$
\begin{gathered}
\bar{\partial}+A(-\infty, \cdot): W_{N^{*} V_{0}, N^{*} V_{0}^{\prime}}^{1, p}\left(\Sigma, \mathbb{C}^{n}\right) \rightarrow L^{p}\left(\Sigma, \mathbb{C}^{n}\right), \\
\bar{\partial}+A(+\infty, \cdot): W_{N^{*} V_{k}, N^{*} V_{k^{\prime}}^{\prime}}^{1, p}\left(\Sigma, \mathbb{C}^{n}\right) \rightarrow L^{p}\left(\Sigma, \mathbb{C}^{n}\right),
\end{gathered}
$$

are invertible. Since invertibility is an open condition in the operator norm, there exist $s>\max |\operatorname{Re} \mathscr{S}|+2$ and $c_{1}>0$ such that for any $u \in X_{\mathscr{Y}, \mathscr{Y}, \mathscr{W}^{\prime}}^{1, p}\left(\Sigma, \mathbb{C}^{n}\right)$ with support disjoint from $\{|\operatorname{Re} z| \leq s-1\}$ there holds

$$
\|u\|_{X^{1, p}(\Sigma)}=\|u\|_{W^{1, p}(\Sigma)} \leq c_{1}\|(\bar{\partial}+A) u\|_{L^{p}(\Sigma)}=c_{1}\|(\bar{\partial}+A) u\|_{X^{p}(\Sigma)} .
$$


By Lemma 5.10, there exists $c_{2}>0$ such that for every $u \in X_{\mathscr{S}, \mathscr{V}, \mathscr{V}^{\prime}}^{1, p}\left(\Sigma, \mathbb{C}^{n}\right)$ with support in $\{|\operatorname{Re} z| \leq s\}$ there holds

$$
\begin{aligned}
\|u\|_{X^{1, p}(\Sigma)} & \leq c_{2}\left(\|u\|_{X^{p}(\Sigma)}+\|\bar{\partial} u\|_{X^{p}(\Sigma)}\right) \\
& \leq\left(c_{2}+\|A\|_{\infty}\right)\|u\|_{X^{p}(\Sigma)}+c_{2}\|(\bar{\partial}+A) u\|_{X^{p}(\Sigma)} .
\end{aligned}
$$

The inequality (5-22) easily follows from (5-23) and (5-24) by writing any $u \in$ $X_{\mathscr{S}, \mathscr{V}, \mathscr{V}^{\prime}}^{1, p}\left(\Sigma, \mathbb{C}^{n}\right)$ as $u=(1-\varphi) u+\varphi u$, for $\varphi$ a smooth real function on $\Sigma$ having support in $\{|\operatorname{Re} z|<s\}$ and such that $\varphi=1$ on $\{|\operatorname{Re} z| \leq s-1\}$.

Finally, by Proposition 5.13 the linear operator

$$
X_{\mathscr{S}, \mathscr{V}, \mathscr{V}^{\prime}}^{1, p}\left(\Sigma, \mathbb{C}^{n}\right) \rightarrow X_{\mathscr{\varphi}}^{p}\left(\Sigma, \mathbb{C}^{n}\right), \quad u \mapsto \chi_{s} u,
$$

is compact. Therefore the estimate (5-22) implies that $\bar{\partial}_{A}$ has finite dimensional kernel and closed range, that is it is semi-Fredholm with index less than $+\infty$.

It would not be difficult to use the regularity of weak solutions of the Cauchy-Riemann operator to prove that the cokernel of $\bar{\partial}_{A}$ is finite-dimensional, so that $\bar{\partial}_{A}$ is Fredholm. However, this will follow directly from the index computation presented in the next section.

\subsection{A Liouville type result}

Let us consider the following particular case in dimension $n=1$ :

$$
k=1, \quad k^{\prime}=0, \quad \mathscr{S}=\{0\}, \quad V_{0}=(0), \quad V_{1}=\mathbb{R}, \quad V_{0}^{\prime}=\mathbb{R}, \quad A(z)=\alpha,
$$

with $\alpha$ a real number. In other words, we are looking at the operator $\bar{\partial}+\alpha$ on a space of $\mathbb{C}$-valued maps $u$ on $\Sigma$ such that $u(s)$ is purely imaginary for $s \leq 0, u(s)$ is real for $s \geq 0$, and $u(s+i)$ is real for every $s \in \mathbb{R}$. Notice that $\Phi^{-}(t)=\Phi^{+}(t)=e^{i \alpha t}$, so

$$
e^{i \alpha} i \mathbb{R} \cap \mathbb{R}=(0) \quad \forall \alpha \in \mathbb{R} \backslash(\pi / 2+\pi \mathbb{Z}), \quad e^{i \alpha} \mathbb{R} \cap \mathbb{R}=(0) \quad \forall \alpha \in \mathbb{R} \backslash \pi \mathbb{Z},
$$

so the assumptions of Theorem 5.9 are satisfied whenever $\alpha$ is not an integer multiple of $\pi / 2$. In order to simplify the notation, we set

$$
X^{p}(\Sigma):=X_{\{0\}}^{p}(\Sigma, \mathbb{C}), \quad X^{1, p}(\Sigma):=X_{\{0\},((0), \mathbb{R}),(\mathbb{R})}^{1, p}(\Sigma, \mathbb{C}) .
$$

We start by studying the regularity of the elements of the kernel of $\bar{\partial}_{\alpha}$ :

Lemma 5.15 Let $p>1$ and $\alpha \in \mathbb{R} \backslash(\pi / 2) \mathbb{Z}$. If $u$ belongs to the kernel of

$$
\bar{\partial}_{\alpha}: X^{1, p}(\Sigma) \rightarrow X^{p}(\Sigma),
$$


then $u$ is smooth on $\Sigma \backslash\{0\}$, it satisfies the boundary conditions pointwise, and the function $(\mathscr{R} u)(\zeta)=u\left(\zeta^{2}\right)$ is smooth on $\mathrm{Cl}\left(\mathbb{H}^{+}\right) \cap \mathbb{D}_{1}$. In particular, $u$ is continuous at 0 , and $D u(z)=O\left(|z|^{-1 / 2}\right)$ for $z \rightarrow 0$.

Proof The regularity theory for weak solutions of $\bar{\partial}$ on $\mathbb{C}$ and on the half-plane $\mathbb{H}$ (Theorem 5.4) implies - by a standard bootstrap argument - that $u \in C^{\infty}(\Sigma \backslash\{0\})$. We just need to check the regularity of $u$ at 0 .

Consider the function $f(\zeta):=e^{\alpha \bar{\zeta}^{2} / 2} u\left(\zeta^{2}\right)$ on $\mathbb{H}^{+} \cap \mathbb{D}_{1}$. Since

$$
\bar{\partial} f(\zeta)=2 \bar{\zeta} e^{\alpha \bar{\zeta}^{2} / 2}\left(\bar{\partial} u\left(\zeta^{2}\right)+\alpha u\left(\zeta^{2}\right)\right)=0,
$$

$f$ is holomorphic on $\mathbb{H}^{+} \cap \mathbb{D}_{1}$. Moreover, by (5-16) the function $f$ belongs to $W^{1, p}\left(\mathbb{H}^{+} \cap \mathbb{D}_{1}\right)$, and in particular it is square integrable. The function $f$ is real on $\mathbb{R}^{+}$and purely imaginary on $i \mathbb{R}^{+}$, so a double Schwarz reflection produces a holomorphic extension of $f$ to $\mathbb{D}_{1} \backslash\{0\}$. Such an extension of $f$ is still square integrable, so the singularity 0 is removable and the function is holomorphic on the whole $\mathbb{D}_{1}$. It follows that

$$
(\mathscr{R} u)(\zeta)=u\left(\zeta^{2}\right)=e^{-\alpha \bar{\zeta}^{2} / 2} f(\zeta)
$$

is smooth on $\mathrm{Cl}\left(\mathbb{H}^{+}\right) \cap \mathbb{D}_{1}$, as claimed.

The real Banach space $X^{p}(\Sigma)$ is the space of $L^{p}$ functions with respect to the measure defined by the density

$$
\rho_{p}(z):= \begin{cases}1 & \text { if } z \in \Sigma \backslash \mathbb{D}_{r} \\ |z|^{p / 2-1} & \text { if } z \in \Sigma \cap \mathbb{D}_{r} .\end{cases}
$$

So the dual of $X^{p}(\Sigma)$ can be identified with the real Banach space

$$
\left\{\left.v \in L_{\mathrm{loc}}^{1}(\Sigma, \mathbb{C})\left|\int_{\Sigma}\right| v\right|^{q} \rho_{p}(z) d s d t<+\infty\right\}, \quad \text { where } \frac{1}{p}+\frac{1}{q}=1,
$$

by using the duality paring

$$
\left(X^{p}(\Sigma)\right)^{*} \times X^{p}(\Sigma) \rightarrow \mathbb{R}, \quad(v, u) \mapsto \operatorname{Re} \int_{\Sigma}\langle v, u\rangle \rho_{p}(z) d s d t .
$$

We prefer to use the standard duality pairing

$$
\left(X^{p}(\Sigma)\right)^{*} \times X^{p}(\Sigma) \rightarrow \mathbb{R}, \quad(w, u) \mapsto \operatorname{Re} \int_{\Sigma}\langle w, u\rangle d s d t
$$


With the latter choice, the dual of $X^{p}(\Sigma)$ is identified with the space of functions $w=\rho_{p}(z) v$, where $v$ varies in the space (5-25). From $1 / p+1 / q=1$ we get the identity

$$
\begin{aligned}
\|w\|_{X^{q}}^{q} & =\int_{\Sigma \backslash \mathbb{D}_{r}}|w|^{q} d s d t+\int_{\Sigma \cap \mathbb{D}_{r}}|w|^{q}|z|^{q / 2-1} d s d t \\
& =\int_{\Sigma \backslash \mathbb{D}_{r}}|v|^{q} d s d t+\int_{\Sigma \cap \mathbb{D}_{r}}|v|^{q}|z|^{(p / 2-1) q}|z|^{q / 2-1} d s d t \\
& =\int_{\Sigma \backslash \mathbb{D}_{r}}|v|^{q} d s d t+\int_{\Sigma \cap \mathbb{D}_{r}}|v|^{q}|z|^{p / 2-1} d s d t=\int_{\Sigma}|v|^{q} \rho_{p}(z) d s d t,
\end{aligned}
$$

which shows that the standard duality paring (5-26) produces the identification

$$
\left(X^{p}(\Sigma)\right)^{*} \cong X^{q}(\Sigma), \quad \text { for } \frac{1}{p}+\frac{1}{q}=1 .
$$

Therefore, we view the cokernel of $\bar{\partial}_{\alpha}: X^{1, p}(\Sigma) \rightarrow X^{p}(\Sigma)$ as a subspace of $X^{q}(\Sigma)$. Its elements are a priori less regular at 0 than the elements of the kernel:

Lemma 5.16 Let $p>1$ and $\alpha \in \mathbb{R} \backslash(\pi / 2) \mathbb{Z}$. If $v \in X^{q}(\Sigma), 1 / p+1 / q=1$, belongs to the cokernel of

$$
\bar{\partial}_{\alpha}: X^{1, p}(\Sigma) \rightarrow X^{p}(\Sigma),
$$

then $v$ is smooth on $\Sigma \backslash\{0\}$, it solves the equation $\partial v-\alpha v=0$ with boundary conditions

$$
\begin{array}{cc}
v(s) \in \mathbb{R}, \quad v(-s) \in i \mathbb{R} & \forall s>0, \\
v(s+i) \in \mathbb{R} & \forall s \in \mathbb{R},
\end{array}
$$

and the function $(\mathscr{T} v)(\zeta)=2 \bar{\zeta} v\left(\zeta^{2}\right)$ is smooth on $\mathrm{Cl}\left(\mathbb{H}^{+}\right) \cap \mathbb{D}_{1}$. In particular, $v(z)=$ $O\left(|z|^{-1 / 2}\right)$ and $D v(z)=O\left(|z|^{-3 / 2}\right)$ for $z \rightarrow 0$.

Proof Since $v \in X^{q}(\Sigma)$ annihilates the image of $\bar{\partial}_{\alpha}$, there holds

$$
\operatorname{Re} \int_{\Sigma}\langle v(z), \bar{\partial} u(z)+\alpha u(z)\rangle d s d t=0
$$

for every $u \in X^{1, p}(\Sigma)$. By letting $u$ vary among all smooth functions in $X^{1, p}(\Sigma)$ which are compactly supported in $\Sigma \backslash\{0\}$, the regularity theory for weak solutions of $\partial$ (the analogue of Theorem 5.4) and a bootstrap argument show that $v$ is smooth on $\Sigma \backslash\{0\}$ and it solves the equation $\partial v-\alpha v=0$ with boundary conditions (5-27). It remains to study the regularity of $v$ at 0 . 
Set $w(\zeta):=(\mathcal{T} v)(\zeta)=2 \bar{\zeta} v\left(\zeta^{2}\right)$. By (5-14), the function $w$ is in $L^{q}\left(\mathbb{H}^{+} \cap \mathbb{D}_{1}\right)$. Let $\varphi \in C_{c}^{\infty}\left(\mathrm{Cl}\left(\mathbb{H}^{+}\right) \cap \mathbb{D}_{1}\right)$ be real on $\mathbb{R}^{+}$and purely imaginary on $i \mathbb{R}^{+}$. Then the function $u$ defined by $u\left(\zeta^{2}\right)=\varphi(\zeta)$ belongs to $X^{1, p}(\Sigma)$, and by (5-28) we have

$$
\begin{aligned}
& 0=\operatorname{Re} \int_{\Sigma}\langle v, \bar{\partial} u+\alpha u\rangle d s d t=4 \operatorname{Re} \int_{\mathbb{H}^{+} \cap \mathbb{D}_{1}}\left|\zeta^{2}\right|\left\langle\frac{1}{2 \bar{\zeta}} w(\zeta), \frac{1}{2 \bar{\zeta}} \bar{\partial} \varphi(\zeta)+\alpha \varphi(\zeta)\right\rangle d \sigma d \tau \\
& =\operatorname{Re} \int_{\mathbb{H}^{+} \cap \mathbb{D}_{1}}\langle w(\zeta), \bar{\partial} \varphi(\zeta)+2 \alpha \bar{\zeta} \varphi(\zeta)\rangle d \sigma d \tau \text {. }
\end{aligned}
$$

The above identity can be rewritten as

$$
\operatorname{Re} \int_{\mathbb{H}^{+} \cap \mathbb{D}_{1}}\langle w(\zeta), \bar{\partial} \varphi(\zeta)\rangle d \sigma d \tau=-\operatorname{Re} \int_{\mathbb{H}^{+} \cap \mathbb{D}_{1}}\langle 2 \alpha \zeta w(\zeta), \varphi(\zeta)\rangle d \sigma d \tau
$$

so $w$ is a weak solution of $\partial w=2 \alpha \zeta w$ on $\mathrm{Cl}\left(\mathbb{H}^{+}\right) \cap \mathbb{D}_{1}$ with real boundary conditions. Since $w$ is in $L^{q}\left(\mathbb{H}^{+} \cap \mathbb{D}_{1}\right)$, Lemma 5.7 implies that $w$ is in $W^{1, q}\left(\mathbb{H}^{+} \cap \mathbb{D}_{1}\right)$. In particular, $w$ is square integrable on $\mathbb{H}^{+} \cap \mathbb{D}_{1}$, and so is the function

$$
f(\zeta):=e^{-\alpha \zeta^{2} / 2} w(\zeta)
$$

The function $f$ is antiholomorphic, it takes real values on $\mathbb{R}^{+}$and on $i \mathbb{R}^{+}$, so by a double Schwarz reflection it can be extended to an antiholomorphic function on $\mathbb{D}_{1} \backslash\{0\}$. Since $f$ is square integrable, the singularity 0 is removable and $f$ is antiholomorphic on $\mathbb{D}_{1}$. Therefore

$$
(\mathscr{T} v)(\zeta)=w(\zeta)=e^{\alpha \zeta^{2} / 2} f(\zeta)
$$

is smooth on $\mathrm{Cl}\left(\mathbb{H}^{+}\right) \cap \mathbb{D}_{1}$, as claimed.

We can finally prove the following Liouville type result:

Proposition 5.17 If $0<\alpha<\pi / 2$, the operator

$$
\bar{\partial}_{\alpha}: X^{1, p}(\Sigma) \rightarrow X^{p}(\Sigma)
$$

is an isomorphism, for every $1<p<\infty$.

Proof By Proposition 5.14 the operator $\bar{\partial}_{\alpha}$ is semi-Fredholm, so it is enough to prove that its kernel and cokernel are both $(0)$.

Let $u \in X^{1, p}(\Sigma)$ be an element of the kernel of $\bar{\partial}_{\alpha}$. By Proposition 5.12, $u(z)$ has exponential decay for $|\operatorname{Re} z| \rightarrow+\infty$ together with all its derivatives. By Lemma 5.15, $u$ is smooth on $\Sigma \backslash\{0\}$, it is continuous at 0 , and $D u(z)=O\left(|z|^{-1 / 2}\right.$ ) for $z \rightarrow 0$. 
Then the function $w:=u^{2}$ belongs to $W^{1, q}(\Sigma, \mathbb{C})$ for every $q<4$. Moreover, $w$ is real on the boundary of $\Sigma$, and it satisfies the equation

$$
\bar{\partial} w+2 \alpha w=0 \text {. }
$$

Since $0<2 \alpha<\pi, e^{2 \alpha i} \mathbb{R} \cap \mathbb{R}=(0)$, so the assumptions of Theorem 5.11 (ii) are satisfied, and the operator

$$
\bar{\partial}_{2 \alpha}: W_{\mathbb{R}, \mathbb{R}}^{1, q}(\Sigma, \mathbb{C}) \rightarrow L^{q}(\Sigma, \mathbb{C})
$$

is an isomorphism. Therefore $w=0$, hence $u=0$, proving that the operator $\bar{\partial}_{\alpha}$ has vanishing kernel.

Let $v \in X^{q}(\Sigma), 1 / p+1 / q=1$, be an element of the cokernel of $\bar{\partial}_{\alpha}$. By Lemma 5.16, $v$ is smooth on $\Sigma \backslash\{0\}, v(s) \in i \mathbb{R}$ for $s<0, v(s) \in \mathbb{R}$ for $s>0, v$ solves $\partial v-\alpha v=0$, and the function

$$
w(\zeta):=2 \bar{\zeta} v\left(\zeta^{2}\right)
$$

is smooth in $\mathrm{Cl}\left(\mathbb{H}^{+}\right) \cap \mathbb{D}_{1}$ and real on the boundary of $\mathbb{H}^{+}$. In particular, $v(z)=$ $O\left(|z|^{-1 / 2}\right)$ and $D v(z)=O\left(|z|^{-3 / 2}\right)$ for $z \rightarrow 0$. Furthermore, by Proposition 5.12, $v$ and $D v$ decay exponentially for $|\operatorname{Re} z| \rightarrow+\infty$. More precisely, since the spectrum of the operator $L_{\alpha}$ on $L^{2}([0,1], \mathbb{C})$,

$\operatorname{dom} L_{\alpha}=W_{\mathbb{R}, \mathbb{R}}^{1,2}([0,1], \mathbb{C})=\left\{u \in W^{1,2}([0,1], \mathbb{C}) \mid u(0), u(1) \in \mathbb{R}\right\}, \quad L_{\alpha}=i \frac{d}{d t}+\alpha$, is $\alpha+\pi \mathbb{Z}$, we have $\min \sigma\left(L_{\alpha}\right) \cap[0,+\infty)=\alpha$, hence

$$
|v(z)| \leq c e^{-\alpha|\operatorname{Re} z|} \quad \text { for }|\operatorname{Re} z| \geq 1 .
$$

If $w(0)=0$, the function $v$ vanishes at 0 , and $D v(z)=O\left(|z|^{-1}\right)$ for $z \rightarrow 0$, so $v^{2}$ belongs to $W_{\mathbb{R}, \mathbb{R}}^{1, q}(\Sigma, \mathbb{C})$ for any $q<2$, it solves $\partial v^{2}-2 \alpha v^{2}=0$, and as before we deduce that $v=0$. Therefore, we can assume that the real number $w(0)$ is not zero.

Consider the function

$$
f: \Sigma \backslash\{0\} \rightarrow \mathbb{C}, \quad f(z):=e^{-\alpha \bar{z} / 2} \bar{v}(z) .
$$

Since $\partial v=\alpha v$,

$$
\bar{\partial} f(z)=-\alpha e^{-\alpha \bar{z} / 2} \bar{v}(z)+e^{-\alpha \bar{z} / 2} \overline{\partial v}(z)=e^{-\alpha \bar{z} / 2}(-\alpha \bar{v}(z)+\overline{\alpha v}(z))=0,
$$

so $f$ is holomorphic on the interior of $\Sigma$. Moreover, $f$ is smooth on $\Sigma \backslash\{0\}$, and (5-31) $f(s)=e^{-\alpha s / 2} \bar{v}(s) \in i \mathbb{R} \quad$ for $s<0, \quad f(s)=e^{-\alpha s / 2} \bar{v}(s) \in \mathbb{R} \quad$ for $s>0$,

$$
f(s+i)=e^{-\alpha s / 2} \bar{v}(s+i) e^{\alpha i / 2} \in e^{\alpha i / 2} \mathbb{R} \quad \text { for every } s \in \mathbb{R} .
$$


Denote by $\sqrt{z}$ the determination of the square root on $\mathbb{C} \backslash \mathbb{R}^{-}$such that $\sqrt{z}$ is real and positive for $z$ real and positive, so that $\overline{\sqrt{z}}=\sqrt{\bar{z}}$. By (5-29),

$$
f(z)=-e^{-\alpha \bar{z} / 2} \frac{1}{\sqrt{z}} \bar{w}(\sqrt{z})=\frac{\bar{w}(0)}{\sqrt{z}}+o\left(|z|^{-1 / 2}\right) \quad \text { for } z \rightarrow 0 .
$$

Finally, by (5-30),

$$
\lim _{|\operatorname{Re} z| \rightarrow+\infty} f(z)=0 .
$$

We claim that a holomorphic function with the properties listed above is necessarily zero. By (5-33), setting $z=\rho e^{\theta i}$ with $\rho>0$ and $0 \leq \theta \leq \pi$,

$$
f(z)=\frac{\bar{w}(0)}{\sqrt{\rho}} e^{-\theta i / 2}+o\left(|\rho|^{-1 / 2}\right) \quad \text { for } \rho \rightarrow 0 .
$$

Since $\bar{w}(0)$ is real and not zero, the above expansion at 0 shows that there exists $\rho>0$ such that

$$
f(z) \in \bigcup_{\theta \in]-\pi / 2-\alpha / 4, \alpha / 4[} e^{\theta i} \mathbb{R}, \quad \forall z \in\left(B_{\rho}(0) \cap \Sigma\right) \backslash\{0\} .
$$

If $f=0$ on $\mathbb{R}+i$, then $f$ is identically zero (by reflection and by analytic continuation), so we may assume that $f(\mathbb{R}+i) \neq\{0\}$. By (5-32) the set $f(\mathbb{R}+i)$ is contained in $\mathbb{R} e^{\alpha i / 2}$. Since $f$ is holomorphic on $\operatorname{Int}(\Sigma)$, it is open on such a domain, so we can find $\gamma \in] \alpha / 4, \alpha / 2[\cup] \alpha / 2,3 \alpha / 4\left[\right.$ such that $f(\operatorname{Int}(\Sigma)) \cap \mathbb{R} e^{\gamma i} \neq\{0\}$. By (5-34) and (5-35) there exists $z \in \Sigma \backslash B_{\rho}(0)$ such that

$$
f(z) \in \mathbb{R} e^{\gamma i}, \quad|f(z)|=\sup \left|f(\Sigma \backslash\{0\}) \cap \mathbb{R} e^{\gamma i}\right|>0 .
$$

By (5-31) and (5-32), $z$ belongs to $\operatorname{Int}(\Sigma)$, but since $f$ is open on $\operatorname{Int}(\Sigma)$ this fact contradicts (5-36). Hence $f=0$. Therefore $v$ vanishes on $\Sigma$, concluding the proof of the invertibility of the operator $\bar{\partial}_{\alpha}$.

If we change the sign of $\alpha$ and we invert the boundary conditions on $\mathbb{R}$ we still get an isomorphism. Indeed, if we set $v(s, t):=\bar{u}(-s, t)$ we have

$$
\bar{\partial}_{-\alpha} v(s, t)=\bar{\partial} v(s, t)-\alpha v(s, t)=-\overline{\bar{\partial} u(-s, t)+\alpha u(-s, t)}=-\overline{\partial_{\alpha} u(-s, t)},
$$

so the operators

$$
\begin{gathered}
\bar{\partial}_{\alpha}: X_{\{0\},((0), \mathbb{R}),(\mathbb{R})}^{1, p}(\Sigma, \mathbb{C}) \rightarrow X_{\{0\}}^{p}(\Sigma, \mathbb{C}), \\
\bar{\partial}_{-\alpha}: X_{\{0\},(\mathbb{R},(0)),(\mathbb{R})}^{1, \nu}(\Sigma, \mathbb{C}) \rightarrow X_{\{0\}}^{p}(\Sigma, \mathbb{C})
\end{gathered}
$$

are conjugated. Therefore Proposition 5.17 implies: 
Proposition 5.18 If $0<\alpha<\pi / 2$, the operator

$$
\bar{\partial}_{-\alpha}: X_{\{0\},(\mathbb{R},(0)),(\mathbb{R})}^{1, p}(\Sigma, \mathbb{C}) \rightarrow X_{\{0\}}^{p}(\Sigma, \mathbb{C})
$$

is an isomorphism.

\subsection{Computation of the index}

The computation of the Fredholm index of $\bar{\partial}_{A}$ is based on the Liouville type results proved in the previous section, together with the following additivity formula:

Proposition 5.19 Assume that $A, A_{1}, A_{2} \in C^{0}\left(\overline{\mathbb{R}} \times[0,1], \mathrm{L}\left(\mathbb{R}^{2 n}, \mathbb{R}^{2 n}\right)\right)$ satisfy

$$
\begin{aligned}
& A_{1}(+\infty, t)=A_{2}(-\infty, t), \quad A(-\infty, t)=A_{1}(-\infty, t), \\
& A(+\infty, t)=A_{2}(+\infty, t), \quad \forall t \in[0,1] .
\end{aligned}
$$

Let $\mathscr{V}_{1}=\left(V_{0}, \ldots, V_{k}\right), \mathscr{V}_{2}=\left(V_{k}, \ldots, V_{k+h}\right), \mathscr{V}_{1}^{\prime}=\left(V_{0}^{\prime}, \ldots, V_{k^{\prime}}^{\prime}\right), \mathscr{V}_{2}^{\prime}=\left(V_{k^{\prime}}, \ldots\right.$, $\left.V_{k^{\prime}+h^{\prime}}^{\prime}\right)$ be finite ordered sets of linear subspaces of $\mathbb{R}^{n}$ such that $V_{j}$ and $V_{j+1}, V_{j}^{\prime}$ and $V_{j+1}^{\prime}$ are partially orthogonal, for every $j . \operatorname{Set}^{\mathscr{V}}=\left(V_{0}, \ldots, V_{k}, V_{k+1}, \ldots, V_{k+h}\right)$ and $\mathscr{V}^{\prime}=\left(V_{0}^{\prime}, \ldots, V_{k^{\prime}}^{\prime}, V_{k^{\prime}+1}^{\prime}, \ldots, V_{k^{\prime}+h^{\prime}}^{\prime}\right)$. Assume that $\left(A_{1}, \mathscr{V}_{1}, \mathscr{V}_{1}^{\prime}\right)$ and $\left(A_{2}, \mathscr{V}_{2}, \mathscr{V}_{2}^{\prime}\right)$ satisfy the assumptions of Theorem 5.9. Let $\mathscr{S}_{1}$ be a set consisting of $k$ points in $\mathbb{R}$ and $k^{\prime}$ points in $i+\mathbb{R}$, let $\mathscr{S}_{2}$ be a set consisting of $h$ points in $\mathbb{R}$ and $h^{\prime}$ points in $i+\mathbb{R}$, and let $\mathscr{S}$ be a set consisting of $k+h$ points in $\mathbb{R}$ and $k^{\prime}+h^{\prime}$ points in $i+\mathbb{R}$. For $p \in] 1,+\infty[$ consider the semi-Fredholm operators

$$
\begin{aligned}
& \bar{\partial}_{A_{1}}: X_{\mathscr{S}_{1}, \mathscr{V}_{1}, \mathscr{V}_{1}^{\prime}}^{1, p}\left(\Sigma, \mathbb{C}^{n}\right) \rightarrow X_{\mathscr{S}_{1}}^{p}\left(\Sigma, \mathbb{C}^{n}\right), \quad \bar{\partial}_{A_{2}}: X_{\mathscr{S}_{2}, \mathscr{V}_{2}, \mathscr{V}_{2}^{\prime}}^{1, p}\left(\Sigma, \mathbb{C}^{n}\right) \rightarrow X_{\mathscr{S}_{2}}^{p}\left(\Sigma, \mathbb{C}^{n}\right) \\
& \bar{\partial}_{A}: X_{\mathscr{Y}, \mathscr{Y}, \mathscr{V}^{\prime}}^{1, p}\left(\Sigma, \mathbb{C}^{n}\right) \rightarrow X_{\mathscr{Y}}^{p}\left(\Sigma, \mathbb{C}^{n}\right) .
\end{aligned}
$$

Then

$$
\text { ind } \bar{\partial}_{A}=\text { ind } \bar{\partial}_{A_{1}}+\text { ind } \bar{\partial}_{A_{1}} \text {. }
$$

The proof is analogous to the proof of Theorem 3.2.12 in [46], and we omit it. When there are no jumps, that is $\mathscr{Y}=\varnothing$ and $\mathscr{V}=(V), \mathscr{V}^{\prime}=\left(V^{\prime}\right)$, Theorem 5.11 shows that the index of the operator

$$
\bar{\partial}_{A}: X_{\varnothing,(V),\left(V^{\prime}\right)}^{1, p}\left(\Sigma, \mathbb{C}^{n}\right)=W_{N^{*} V, N^{*} V^{\prime}}^{1, p}\left(\Sigma, \mathbb{C}^{n}\right) \rightarrow L^{p}\left(\Sigma, \mathbb{C}^{n}\right)=X_{\varnothing}^{p}\left(\Sigma, \mathbb{C}^{n}\right)
$$

is

$$
\text { ind } \bar{\partial}_{A}=\mu\left(\Phi^{-} N^{*} V, N^{*} V^{\prime}\right)-\mu\left(\Phi^{+} N^{*} V, N^{*} V^{\prime}\right) \text {. }
$$

In the general case, Proposition 5.19 shows that

(5-37) $\operatorname{ind}\left(\bar{\partial}_{A}: X_{\mathscr{S}, \mathcal{V}, \mathscr{V}^{\prime}}^{1, p}\left(\Sigma, \mathbb{C}^{n}\right) \rightarrow X_{\mathscr{S}}^{p}\left(\Sigma, \mathbb{C}^{n}\right)\right)$

$$
=\mu\left(\Phi^{-} N^{*} V_{0}, N^{*} V_{0}^{\prime}\right)-\mu\left(\Phi^{+} N^{*} V_{k}, N^{*} V_{k^{\prime}}^{\prime}\right)+c\left(V_{0}, \ldots, V_{k} ; V_{0}^{\prime}, \ldots, V_{k^{\prime}}^{\prime}\right) \text {, }
$$


where the correction term $c$ satisfies the additivity formula

$$
\begin{aligned}
& c\left(V_{0}, \ldots, V_{k+h} ; V_{0}^{\prime}, \ldots, V_{k^{\prime}+h^{\prime}}^{\prime}\right) \\
& \quad=c\left(V_{0}, \ldots, V_{k} ; V_{0}^{\prime}, \ldots, V_{k^{\prime}}^{\prime}\right)+c\left(V_{k}, \ldots, V_{k+h} ; V_{k^{\prime}}^{\prime}, \ldots, V_{k^{\prime}+h^{\prime}}^{\prime}\right) \cdot A
\end{aligned}
$$

Since the Maslov index is in general a half-integer, and since we have not proved that the cokernel of $\bar{\partial}_{A}$ is finite dimensional, the correction term $c$ takes values in $(1 / 2) \mathbb{Z} \cup\{-\infty\}$. Actually, the analysis of this section shows that $c$ is always finite, proving that $\bar{\partial}_{A}$ is Fredholm.

Clearly, we have the following direct sum formula

$$
\begin{aligned}
c\left(V_{0} \oplus W_{0}, \ldots, V_{k} \oplus W_{k} ; V_{0}^{\prime} \oplus W_{0}^{\prime}, \ldots, V_{k^{\prime}}^{\prime} \oplus W_{k^{\prime}}^{\prime}\right) \\
\quad=c\left(V_{0}, \ldots, V_{k} ; V_{0}^{\prime}, \ldots, V_{k^{\prime}}^{\prime}\right)+c\left(W_{0}, \ldots, W_{k} ; W_{0}^{\prime}, \ldots, W_{k^{\prime}}^{\prime}\right) .
\end{aligned}
$$

Note also the index formula of Theorem 5.11 produces a correction term of the form

$$
c(\lambda ; \nu)=\mu(\lambda, v),
$$

where $\lambda$ and $v$ are asymptotically constant paths of Lagrangian subspaces on $\mathbb{C}^{n}$. The Liouville type results of the previous section imply that

$$
c\left((0), \mathbb{R}^{n} ; \mathbb{R}^{n}\right)=-n / 2=c\left(\mathbb{R}^{n},(0) ; \mathbb{R}^{n}\right) .
$$

Indeed, by Proposition 5.17 the operator

$$
\bar{\partial}_{\alpha I}: X_{\{0\},\left((0), \mathbb{R}^{n}\right),\left(\mathbb{R}^{n}\right)}^{1, p}\left(\Sigma, \mathbb{C}^{n}\right) \rightarrow X_{\{0\}}^{p}\left(\Sigma, \mathbb{C}^{n}\right)
$$

is an isomorphism if $0<\alpha<\pi / 2$. By (5-5), the Maslov index of the path $e^{i \alpha t} \mathbb{R}^{n}$, $t \in[0,1]$, with respect to $\mathbb{R}^{n}$ is $-n / 2$. On the other hand, the Maslov index of the path $e^{i \alpha t} i \mathbb{R}^{n}, t \in[0,1]$, with respect to $\mathbb{R}^{n}$ is 0 because the intersection is (0) for every $t \in[0,1]$. Inserting the information about the Fredholm and the Maslov index in (5-37), we find

$$
0=\operatorname{ind} \bar{\partial}_{\alpha I}=n / 2+c\left((0), \mathbb{R}^{n} ;(0)\right),
$$

which implies the first identity in (5-41). The second one is proved in the same way by using Proposition 5.18.

Lemma 5.20 Let $\left(V_{0}, V_{1}, \ldots, V_{k}\right)$ be a $(k+1)$-tuple of linear subspaces of $\mathbb{R}^{n}$, with $V_{j-1}$ and $V_{j}$ partially orthogonal for every $j=1, \ldots, k$, and let $W$ be a linear 
subspace of $\mathbb{R}^{n}$. Then

$$
\begin{aligned}
c\left(V_{0}, \ldots, V_{k} ; W\right) & =c\left(W ; V_{0}, \ldots, V_{k}\right) \\
& =\frac{1}{2} \sum_{j=1}^{k}\left(\operatorname{dim} V_{j-1}+\operatorname{dim} V_{j}-2 \operatorname{dim} V_{j-1} \cap V_{j}\right) .
\end{aligned}
$$

Proof Let us start by considering the case $W=\mathbb{R}^{n}$. By the additivity formula (5-38),

$$
c\left(V_{0}, \ldots, V_{k} ; \mathbb{R}^{n}\right)=\sum_{j=1}^{k} c\left(V_{j-1}, V_{j} ; \mathbb{R}^{n}\right) .
$$

Since $V_{j-1}$ and $V_{j}$ are partially orthogonal, $\mathbb{R}^{n}$ has an orthogonal splitting $\mathbb{R}^{n}=$ $X_{1}^{j} \oplus X_{2}^{j} \oplus X_{3}^{j} \oplus X_{4}^{j}$ where $V_{j-1}=X_{1}^{j} \oplus X_{2}^{j}$ and $V_{j}=X_{1}^{j} \oplus X_{3}^{j}$. By the direct sum formula (5-39) and by formula (5-41),

$$
\begin{aligned}
c\left(V_{j-1}, V_{j} ; \mathbb{R}^{n}\right)=c\left(X_{1}^{j}, X_{1}^{j} ; X_{1}^{j}\right)+c\left(X_{2}^{j},(0) ; X_{2}^{j}\right) & \\
& +c\left((0), X_{3}^{j} ; X_{3}^{j}\right)+c\left((0),(0) ; X_{4}^{j}\right) \\
=0-\frac{1}{2} \operatorname{dim} X_{2}^{j}-\frac{1}{2} \operatorname{dim} X_{3}^{j}+0 & =-\frac{1}{2} \operatorname{dim} X_{2}^{j} \oplus X_{3}^{j}
\end{aligned}
$$

Since $\quad \operatorname{dim} X_{2}^{j} \oplus X_{3}^{j}=\operatorname{dim} V_{j-1}+\operatorname{dim} V_{j}-2 \operatorname{dim} V_{j-1} \cap V_{j}$,

the formula for $c\left(V_{0}, \ldots, V_{k} ; \mathbb{R}^{n}\right)$ follows.

Now let $\lambda: \mathbb{R} \rightarrow \mathscr{L}(n)$ be a continuous path of Lagrangian subspaces such that $\lambda(s)=$ $\mathbb{R}^{n}$ for $s \leq-1$ and $\lambda(s)=N^{*} W$ for $s \geq 1$. By an easy generalization of the additivity formula (5-38) to the case of nonconstant Lagrangian boundary conditions,

$$
c\left(N^{*} V_{0} ; \lambda\right)+c\left(V_{0}, \ldots, V_{k} ; W\right)=c\left(V_{0}, \ldots, V_{k} ; \mathbb{R}^{n}\right)+c\left(N^{*} V_{k} ; \lambda\right) .
$$

By (5-40), $c\left(N^{*} V_{0} ; \lambda\right)=-\mu\left(\lambda, N^{*} V_{0}\right)$ and $c\left(N^{*} V_{k} ; \lambda\right)=-\mu\left(\lambda, N^{*} V_{k}\right)$, so (5-42) leads to

$$
\begin{aligned}
c\left(V_{0}, \ldots, V^{k} ; W\right) & =c\left(V_{0}, \ldots, V^{k} ; \mathbb{R}^{n}\right)-\left(\mu\left(\lambda, N^{*} V_{k}\right)-\mu\left(\lambda, N^{*} V_{0}\right)\right) \\
& =c\left(V_{0}, \ldots, V^{k} ; \mathbb{R}^{n}\right)-h\left(N^{*} V_{0}, N^{*} V_{k} ; \mathbb{R}^{n}, N^{*} W\right),
\end{aligned}
$$

where $h$ is the Hörmander index. By Proposition 5.2, the above Hörmander index vanishes, so we get the desired formula for $c\left(V_{0}, \ldots, V_{k} ; W\right)$. The formula for $c\left(W ; V_{0}, \ldots, V_{k}\right)$ follows by using the change of variable $v(s, t)=\bar{u}(s, 1-t)$.

The additivity formula (5-38) leads to

$$
c\left(V_{0}, \ldots, V_{k} ; V_{0}^{\prime}, \ldots, V_{k^{\prime}}^{\prime}\right)=c\left(V_{0}, \ldots, V_{k} ; V_{0}^{\prime}\right)+c\left(V_{k} ; V_{0}^{\prime}, \ldots, V_{k^{\prime}}^{\prime}\right),
$$


and the index formula in the general case follows from (5-37) and the above lemma. This concludes the proof of Theorem 5.9.

\subsection{Half-strips with jumping conormal boundary conditions}

This section is devoted to the analogue of Theorem 5.9 on the half-strips

$$
\begin{aligned}
& \Sigma^{+}:=\{z \in \mathbb{C} \mid 0 \leq \operatorname{Im} z \leq 1, \operatorname{Re} z \geq 0\}, \\
& \Sigma^{-}:=\{z \in \mathbb{C} \mid 0 \leq \operatorname{Im} z \leq 1, \operatorname{Re} z \leq 0\} .
\end{aligned}
$$

In the first case, we fix the following data. Let $k, k^{\prime} \geq 0$ be integers, let

$$
0=s_{0}<s_{1}<\cdots<s_{k}<s_{k+1}=+\infty, \quad 0=s_{0}^{\prime}<s_{1}^{\prime}<\cdots<s_{k^{\prime}}^{\prime}<s_{k^{\prime}+1}^{\prime}=+\infty,
$$

be real numbers, and let $W, V_{0}, \ldots, V_{k}, V_{0}^{\prime}, \ldots, V_{k^{\prime}}^{\prime}$ be linear subspaces of $\mathbb{R}^{n}$ such that $V_{j-1}$ and $V_{j}, V_{j-1}^{\prime}$ and $V_{j}^{\prime}, W$ and $V_{0}, W$ and $V_{0}^{\prime}$, are partially orthogonal. We denote by $\mathscr{V}$ the $(k+1)$-tuple $\left(V_{0}, \ldots, V_{k}\right)$, by $\mathscr{V}^{\prime}$ the $\left(k^{\prime}+1\right)$-tuple $\left(V_{0}^{\prime}, \ldots, V_{k^{\prime}}^{\prime}\right)$, and by $\mathscr{S}$ the set $\left\{s_{1}, \ldots, s_{k}, s_{1}^{\prime}+i, \ldots, s_{k^{\prime}}^{\prime}+i\right\}$. The $X^{p}$ and $X^{1, p}$ norms on $\Sigma^{+}$ are defined as in Section 5.3, and so are the spaces $X_{\mathscr{\varphi}}^{p}\left(\Sigma^{+}, \mathbb{C}^{n}\right)$ and $X_{\mathscr{\varphi}}^{1, p}\left(\Sigma^{+}, \mathbb{C}^{n}\right)$. Let $X_{\mathscr{S}, W, \mathscr{q}, \mathscr{V}^{\prime}}^{1, p}\left(\Sigma^{+}, \mathbb{C}^{n}\right)$ be the completion of the space of maps $u \in C_{\mathscr{Y}, c}^{\infty}\left(\Sigma^{+}, \mathbb{C}^{n}\right)$ satisfying the boundary conditions

$$
\begin{gathered}
u(i t) \in N^{*} W, \quad \forall t \in[0,1], \quad u(s) \in N^{*} V_{j}, \quad \forall s \in\left[s_{j}, s_{j+1}\right], \\
u(s+i) \in N^{*} V_{j}^{\prime}, \quad \forall s \in\left[s_{j}^{\prime}, s_{j+1}^{\prime}\right],
\end{gathered}
$$

with respect to the norm $\|u\|_{X^{1, p}\left(\Sigma^{+}\right)}$.

Let $A \in C^{0}\left([0,+\infty] \times[0,1], \mathrm{L}\left(\mathbb{R}^{2 n}, \mathbb{R}^{2 n}\right)\right)$ be such that $A(+\infty, t)$ is symmetric for every $t \in[0,1]$, and denote by $\Phi^{+}:[0,1] \rightarrow \operatorname{Sp}(2 n)$ the solutions of the linear Hamiltonian system

$$
\frac{d}{d t} \Phi^{+}(t)=i A(+\infty, t) \Phi^{+}(t), \quad \Phi^{+}(0)=I .
$$

Then we have:

Theorem 5.21 Assume that $\Phi^{+}(1) N^{*} V_{k} \cap N^{*} V_{k^{\prime}}^{\prime}=(0)$. Then the $\mathbb{R}$-linear bounded operator

$$
\bar{\partial}_{A}: X_{\mathscr{S}, W, \mathscr{q}, \mathscr{V}^{\prime}}^{1, p}\left(\Sigma^{+}, \mathbb{C}^{n}\right) \rightarrow X_{\mathscr{S}}^{p}\left(\Sigma^{+}, \mathbb{C}^{n}\right), \quad \bar{\partial}_{A} u=\bar{\partial} u+A u,
$$


is Fredholm of index

$$
\begin{aligned}
\text { ind } \bar{\partial}_{A}= & \frac{n}{2}-\mu\left(\Phi^{+} N^{*} V_{k}, N^{*} V_{k^{\prime}}^{\prime}\right) \\
& -\frac{1}{2}\left(\operatorname{dim} V_{0}+\operatorname{dim} W-2 \operatorname{dim} V_{0} \cap W\right) \\
& -\frac{1}{2}\left(\operatorname{dim} V_{0}^{\prime}+\operatorname{dim} W-2 \operatorname{dim} V_{0}^{\prime} \cap W\right) \\
& -\frac{1}{2} \sum_{j=1}^{k}\left(\operatorname{dim} V_{j-1}+\operatorname{dim} V_{j}-2 \operatorname{dim} V_{j-1} \cap V_{j}\right) \\
& -\frac{1}{2} \sum_{j=1}^{k^{\prime}}\left(\operatorname{dim} V_{j-1}^{\prime}+\operatorname{dim} V_{j}^{\prime}-2 \operatorname{dim} V_{j-1}^{\prime} \cap V_{j}^{\prime}\right) .
\end{aligned}
$$

Proof The proof of the fact that $\bar{\partial}_{A}$ is semi-Fredholm is analogous to the case of the full strip, treated in Section 5.4. It remains to compute the index. By an additivity formula analogous to (5-38), it is enough to prove (5-43) in the case with no jumps, that is $k=k^{\prime}=0, \mathscr{V}=\left(V_{0}\right), \mathscr{V}^{\prime}=\left(V_{0}^{\prime}\right)$. In this case, we have a formula of the type

$$
\text { ind } \bar{\partial}_{A}=-\mu\left(\Phi^{+} N^{*} V_{0}, N^{*} V_{0}^{\prime}\right)+c\left(W ; V_{0} ; V_{0}^{\prime}\right),
$$

and we have to determine the correction term $c$.

Assume $W=(0)$, so that $N^{*} W=i \mathbb{R}^{n}$. Let us compute the correction term $c$ when $V_{0}$ and $V_{0}^{\prime}$ are either (0) or $\mathbb{R}^{n}$. We can choose the map $A$ to be the constant map $A(s, t)=\alpha I$, for $\alpha \in] 0, \pi / 2\left[\right.$, so that $\Phi^{+}(t)=e^{i \alpha t}$. The Kernel and cokernel of $\bar{\partial}_{\alpha I}$ are easy to determine explicitly, by separating the variables in the corresponding boundary value PDEs:

(i) If $V_{0}=V_{0}^{\prime}=\mathbb{R}^{n}$, then the kernel and cokernel of $\bar{\partial}_{\alpha I}$ are both (0). Since $\mu\left(e^{i \alpha t} \mathbb{R}^{n}, \mathbb{R}^{n}\right)=-n / 2$, we have $c\left((0) ; \mathbb{R}^{n} ; \mathbb{R}^{n}\right)=-n / 2$.

(ii) If $V_{0}=V_{0}^{\prime}=(0)$, then the kernel of $\bar{\partial}_{\alpha I}$ is $i \mathbb{R}^{n} e^{-\alpha s}$, while its cokernel is (0). Since $\mu\left(e^{i \alpha t} i \mathbb{R}^{n}, i \mathbb{R}^{n}\right)=-n / 2$, we have $c((0) ;(0) ;(0))=n / 2$.

(iii) If either $V_{0}=\mathbb{R}^{n}$ and $V_{0}^{\prime}=(0)$, or $V_{0}=(0)$ and $V_{0}^{\prime}=\mathbb{R}^{n}$, then the kernel and cokernel of $\bar{\partial}_{\alpha I}$ are both (0). Since $\mu\left(e^{i \alpha t} \mathbb{R}^{n},(0)\right)=\mu\left(e^{i \alpha t}(0), \mathbb{R}^{n}\right)=0$, we have $c\left((0) ; \mathbb{R}^{n} ;(0)\right)=c\left((0) ;(0) ; \mathbb{R}^{n}\right)=0$.

Now let $W, V_{0}$ and $V_{0}^{\prime}$ be arbitrary (with $W$ partially orthogonal to both $V_{0}$ and $V_{0}^{\prime}$ ). Let $U \in \mathrm{U}(n)$ be such that $U N^{*} W=i \mathbb{R}^{n}$. Then $U N^{*} V_{0}=N^{*} W_{0}$ and $U N^{*} V_{0}^{\prime}=$ $N^{*} W_{0}^{\prime}$, where

$$
W_{0}=\left(V_{0} \cap W\right)^{\perp} \cap\left(V_{0}+W\right), \quad W_{0}^{\prime}=\left(V_{0}^{\prime} \cap W\right)^{\perp} \cap\left(V_{0}^{\prime}+W\right) .
$$


By using the change of variable $v=U u$, we find

$$
c\left(W ; V_{0} ; V_{0}^{\prime}\right)=c\left((0) ; W_{0} ; W_{0}^{\prime}\right),
$$

and we are reduced to compute the latter quantity. By an easy homotopy argument, using the fact that the Fredholm index is locally constant in the operator norm topology, we can assume that $W_{0}$ and $W_{0}^{\prime}$ are partially orthogonal. Then $\mathbb{R}^{n}$ has an orthogonal splitting $\mathbb{R}^{n}=X_{1} \oplus X_{2} \oplus X_{3} \oplus X_{4}$, where

$$
W_{0}=X_{1} \oplus X_{2}, \quad W_{0}^{\prime}=X_{1} \oplus X_{3},
$$

from which

$$
N^{*} W_{0}=X_{1} \oplus X_{2} \oplus i X_{3} \oplus i X_{4}, \quad N^{*} W_{0}^{\prime}=X_{1} \oplus i X_{2} \oplus X_{3} \oplus i X_{4} .
$$

Then the operator $\bar{\partial}_{\alpha I}$ decomposes as the direct sum of four operators, whose index is computed in cases (i), (ii), and (iii) above. Indeed,

$$
\begin{aligned}
c\left((0) ; W_{0} ; W_{0}^{\prime}\right) & =\frac{1}{2} \operatorname{dim} X_{4}-\frac{1}{2} \operatorname{dim} X_{1} \\
& =\frac{1}{2} \operatorname{codim}\left(W_{0}+W_{0}^{\prime}\right)-\frac{1}{2} \operatorname{dim} W_{0} \cap W_{0}^{\prime}=\frac{1}{2}\left(n-\operatorname{dim} W_{0}-\operatorname{dim} W_{0}^{\prime}\right) .
\end{aligned}
$$

Since

$$
\begin{aligned}
& \operatorname{dim} W_{0}=\operatorname{dim}\left(V_{0}+W\right)-\operatorname{dim} V_{0} \cap W=\operatorname{dim} V_{0}+\operatorname{dim} W-2 \operatorname{dim} W_{0} \cap W, \\
& \operatorname{dim} W_{0}^{\prime}=\operatorname{dim}\left(V_{0}^{\prime}+W\right)-\operatorname{dim} V_{0}^{\prime} \cap W=\operatorname{dim} V_{0}^{\prime}+\operatorname{dim} W-2 \operatorname{dim} W_{0}^{\prime} \cap W,
\end{aligned}
$$

we find

$c\left((0) ; W_{0} ; W_{0}^{\prime}\right)=\frac{n}{2}-\frac{1}{2}\left(\operatorname{dim} V_{0}+\operatorname{dim} W-2 \operatorname{dim} W_{0} \cap W\right)$

$-\frac{1}{2}\left(\operatorname{dim} V_{0}^{\prime}+\operatorname{dim} W-2 \operatorname{dim} W_{0}^{\prime} \cap W\right)$.

Together with (5-44), this proves formula (5-43).

We conclude this section by considering the case of the left half-strip $\Sigma^{-}$. Let $k, k^{\prime} \geq 0$, $\mathscr{V}=\left(V_{0}, \ldots, V_{k}\right)$, and $\mathscr{V}^{\prime}=\left(V_{0}^{\prime}, \ldots, V_{k^{\prime}}^{\prime}\right)$ be as above. Let

$$
-\infty=s_{k+1}<s_{k}<\cdots<s_{1}<s_{0}=0, \quad-\infty=s_{k^{\prime}+1}^{\prime}<s_{k^{\prime}}^{\prime}<\cdots<s_{1}^{\prime}<s_{0}^{\prime}=0,
$$

be real numbers, and set $\mathscr{S}=\left\{s_{1}, \ldots, s_{k}, s_{1}^{\prime}+i, \ldots, s_{k^{\prime}}^{\prime}+i\right\}$.

Let $X_{\mathscr{S}, W, \mathscr{V}, \mathscr{V}^{\prime}}^{1, p}\left(\Sigma^{-}, \mathbb{C}^{n}\right)$ be the completion of the space of maps $u \in C_{\mathscr{S}_{, c}}^{\infty}\left(\Sigma^{-}, \mathbb{C}^{n}\right)$ satisfying the boundary conditions

$$
\begin{gathered}
u(i t) \in N^{*} W, \quad \forall t \in[0,1], \quad u(s) \in N^{*} V_{j}, \quad \forall s \in\left[s_{j+1}, s_{j}\right], \\
u(s+i) \in N^{*} V_{j}^{\prime}, \quad \forall s \in\left[s_{j+1}^{\prime}, s_{j}^{\prime}\right],
\end{gathered}
$$

with respect to the norm $\|u\|_{X^{1, p}\left(\Sigma^{-}\right)}$. 
Let $A \in C^{0}\left([-\infty, 0] \times[0,1], \mathrm{L}\left(\mathbb{R}^{2 n}, \mathbb{R}^{2 n}\right)\right)$ be such that $A(-\infty, t)$ is symmetric for every $t \in[0,1]$, and denote by $\Phi^{-}:[0,1] \rightarrow \mathrm{Sp}(2 n)$ the solutions of the linear Hamiltonian system

$$
\frac{d}{d t} \Phi^{-}(t)=i A(-\infty, t) \Phi^{-}(t), \quad \Phi^{-}(0)=I
$$

Then we have:

Theorem 5.22 Assume that $\Phi^{-}(1) N^{*} V_{k} \cap N^{*} V_{k^{\prime}}^{\prime}=(0)$. Then the $\mathbb{R}$-linear operator

$$
\bar{\partial}_{A}: X_{\mathscr{S}, W, \mathscr{V}, \mathscr{V}^{\prime}}^{1, p}\left(\Sigma^{-}, \mathbb{C}^{n}\right) \rightarrow X_{\mathscr{S}}^{p}\left(\Sigma^{-}, \mathbb{C}^{n}\right), \quad \bar{\partial}_{A} u=\bar{\partial} u+A u,
$$

is bounded and Fredholm of index

$$
\begin{aligned}
\text { ind } \bar{\partial}_{A}= & \frac{n}{2}+\mu\left(\Phi^{-} N^{*} V_{k}, N^{*} V_{k^{\prime}}^{\prime}\right)-\frac{1}{2}\left(\operatorname{dim} V_{0}+\operatorname{dim} W-2 \operatorname{dim} V_{0} \cap W\right) \\
& -\frac{1}{2}\left(\operatorname{dim} V_{0}^{\prime}+\operatorname{dim} W-2 \operatorname{dim} V_{0}^{\prime} \cap W\right)
\end{aligned}
$$

$$
\begin{aligned}
& -\frac{1}{2} \sum_{j=1}^{k}\left(\operatorname{dim} V_{j-1}+\operatorname{dim} V_{j}-2 \operatorname{dim} V_{j-1} \cap V_{j}\right) \\
& -\frac{1}{2} \sum_{j=1}^{k^{\prime}}\left(\operatorname{dim} V_{j-1}^{\prime}+\operatorname{dim} V_{j}^{\prime}-2 \operatorname{dim} V_{j-1}^{\prime} \cap V_{j}^{\prime}\right) .
\end{aligned}
$$

Indeed, notice that if $u(s, t)=\bar{v}(-s, t)$, then

$$
-(\bar{\partial} u(s, t)+A(s, t) u(s, t))=C(\bar{\partial} v(-s, t)-C A(s, t) C v(-s, t)),
$$

where $C$ is denotes complex conjugation. Then the operator (5-45) is obtained from the operator

$$
\bar{\partial}_{B}: X_{-\mathscr{Y}, W, \mathscr{V}, \mathscr{V}^{\prime}}^{1, p}\left(\Sigma^{+}, \mathbb{C}^{n}\right) \rightarrow X_{-\mathscr{P}}^{p}\left(\Sigma^{+}, \mathbb{C}^{n}\right), \quad \bar{\partial}_{B} v=\bar{\partial} v+B v,
$$

where $B(s, t)=-C A(-s, t) C$, by left and right multiplication by isomorphisms. In particular, the indices are the same. Then Theorem 5.22 follows from Theorem 5.21, taking into account the fact that the solution $\Phi^{+}$of

$$
\frac{d}{d t} \Phi^{+}(t)=i B(+\infty, t) \Phi^{+}(t), \quad \Phi^{+}(0)=I,
$$

is $\Phi^{+}(t)=C \Phi^{-}(t) C$, so that

$$
\mu\left(\Phi^{+} N^{*} V_{k}, N^{*} V_{k^{\prime}}^{\prime}\right)=\mu\left(C \Phi^{-} C N^{*} V_{k}, N^{*} V_{k^{\prime}}^{\prime}\right)=-\mu\left(\Phi^{-} N^{*} V_{k}, N^{*} V_{k^{\prime}}^{\prime}\right) .
$$




\subsection{Nonlocal boundary conditions}

It is useful to dispose of versions of Theorems 5.9, 5.21 and 5.22, involving nonlocal boundary conditions. In the case of the full strip $\Sigma$, let us fix the following data. Let $k \geq 0$ be an integer, let

$$
-\infty=s_{0}<s_{1}<\cdots<s_{k}<s_{k+1}=+\infty
$$

be real numbers, and set $\mathscr{S}:=\left\{s_{1}, \ldots, s_{k}, s_{1}+i, \ldots, s_{k}+i\right\}$. Let $W_{0}, W_{1}, \ldots, W_{k}$ be linear subspaces of $\mathbb{R}^{n} \times \mathbb{R}^{n}$ such that $W_{j-1}$ and $W_{j}$ are partially orthogonal, for $j=1, \ldots, k$, and set $\mathscr{W}=\left(W_{0}, \ldots, W_{k}\right)$.

The space $X_{\mathscr{Y}, W}^{1, p}\left(\Sigma, \mathbb{C}^{n}\right)$ is defined as the completion of the space of all $u \in C_{\mathscr{Y}, c}^{\infty}\left(\Sigma, \mathbb{C}^{n}\right)$ such that

$$
(u(s), \bar{u}(s+i)) \in N^{*} W_{j}, \quad \forall s \in\left[s_{j}, s_{j+1}\right], \quad j=0, \ldots, k,
$$

with respect to the norm $\|u\|_{X^{1, p}(\Sigma)}$.

Let $A \in C^{0}\left(\overline{\mathbb{R}} \times[0,1], \mathrm{L}\left(\mathbb{R}^{2 n}, \mathbb{R}^{2 n}\right)\right)$ be such that $A( \pm \infty, t) \in \operatorname{Sym}(2 n, \mathbb{R})$ for every $t \in[0,1]$, and define the symplectic paths $\Phi^{+}, \Phi^{-}:[0,1] \rightarrow \operatorname{Sp}(2 n)$ as the solutions of the linear Hamiltonian systems

$$
\frac{d}{d t} \Phi^{ \pm}(t)=i A( \pm \infty, t) \Phi^{ \pm}(t), \quad \Phi^{ \pm}(0)=I .
$$

Denote by $C$ the complex conjugation, and recall from Section 5.1 that $\Phi \in \mathrm{L}\left(\mathbb{R}^{2 n}, \mathbb{R}^{2 n}\right)$ is symplectic if and only if graph $\Phi C$ is a Lagrangian subspace of $\left(\mathbb{R}^{2 n} \times \mathbb{R}^{2 n}, \omega_{0} \times \omega_{0}\right)$. Then we have the following:

Theorem 5.23 Assume that graph $C \Phi^{-}(1) \cap N^{*} W_{0}=(0)$ and graph $C \Phi^{+}(1) \cap$ $N^{*} W_{k}=(0)$. Then for every $\left.p \in\right] 1,+\infty[$ the $\mathbb{R}$-linear operator

$$
\bar{\partial}_{A}: X_{\mathscr{S}, \mathscr{W}}^{1, p}\left(\Sigma, \mathbb{C}^{n}\right) \rightarrow X_{\mathscr{S}}^{p}\left(\Sigma, \mathbb{C}^{n}\right), \quad u \mapsto \bar{\partial} u+A u,
$$

is bounded and Fredholm of index

ind $\bar{\partial}_{A}=\mu\left(\operatorname{graph} \Phi^{-} C, N^{*} W_{0}\right)-\mu\left(\operatorname{graph} \Phi^{+} C, N^{*} W_{k}\right)$

$$
-\frac{1}{2} \sum_{j=1}^{k}\left(\operatorname{dim} W_{j-1}+\operatorname{dim} W_{j}-2 \operatorname{dim} W_{j-1} \cap W_{j}\right) .
$$

Proof Given $u: \Sigma \rightarrow \mathbb{C}^{n}$ define $\tilde{u}: \Sigma \rightarrow \mathbb{C}^{2 n}$ by

$$
\tilde{u}(z):=(u(z / 2), \bar{u}(\bar{z} / 2+i)) .
$$


The map $u \mapsto \tilde{u}$ determines a linear isomorphism

$$
F: X_{\mathscr{Y}, W}^{1, p}\left(\Sigma, \mathbb{C}^{n}\right) \stackrel{\cong}{\longrightarrow} X_{\mathscr{Y}^{\prime}, W, W^{\prime}}^{1, p}\left(\Sigma, \mathbb{C}^{2 n}\right),
$$

where $\mathscr{S}^{\prime}=\left\{2 s_{1}, \ldots, 2 s_{k}, 2 s_{1}+i, \ldots, 2 s_{k}+i\right\}, \mathscr{W}^{\prime}$ is the $(k+1)$-tuple $\left(\Delta_{\mathbb{R}^{n}}, \ldots, \Delta_{\mathbb{R}^{n}}\right)$, and we have used the identity

$$
N^{*} \Delta_{\mathbb{R}^{n}}=\operatorname{graph} C=\left\{(w, \bar{w}) \mid w \in \mathbb{C}^{n}\right\} .
$$

The map $v \mapsto \widetilde{v} / 2$ determines an isomorphism

$$
G: X_{\varphi}^{p}\left(\Sigma, \mathbb{C}^{n}\right) \stackrel{\cong}{\longrightarrow} X_{\varphi^{\prime}}^{p}\left(\Sigma, \mathbb{C}^{2 n}\right) .
$$

The composition $G \circ \bar{\partial}_{A} \circ F^{-1}$ is the operator

$$
\bar{\partial}_{\tilde{A}}: X_{\mathscr{S}^{\prime}, W^{\prime}, W^{\prime}}^{1, p}\left(\Sigma, \mathbb{C}^{2 n}\right) \longrightarrow X_{\mathscr{S}^{\prime}}^{p}\left(\Sigma, \mathbb{C}^{2 n}\right), \quad u \mapsto \bar{\partial} u+\tilde{A} u,
$$

where

$$
\tilde{A}(z):=\frac{1}{2}(A(z / 2) \oplus C A(\bar{z} / 2+i) C) .
$$

Since

$$
\widetilde{A}( \pm \infty, t)=\frac{1}{2}(A( \pm \infty, t / 2) \oplus C A( \pm \infty, 1-t / 2) C),
$$

we easily see that the solutions $\tilde{\Phi}^{ \pm}$of

$$
\frac{d}{d t} \tilde{\Phi}^{ \pm}(t)=i \tilde{A}( \pm \infty, t) \tilde{\Phi}^{ \pm}(t), \quad \tilde{\Phi}^{ \pm}(0)=I,
$$

are given by

$$
\tilde{\Phi}^{ \pm}(t)=\Phi^{ \pm}(t / 2) \oplus C \Phi^{ \pm}(1-t / 2) \Phi(1)^{-1} C .
$$

The above formula implies

$$
\widetilde{\Phi}^{ \pm}(t)^{-1} N^{*} \Delta_{\mathbb{R}^{n}}=\operatorname{graph} C \Phi^{ \pm}(1) \Phi^{ \pm}(1-t / 2)^{-1} \Phi^{ \pm}(t / 2) .
$$

For $t=1$ we get

$$
\begin{aligned}
& \widetilde{\Phi}^{-}(1) N^{*} W_{0} \cap N^{*} \Delta_{\mathbb{R}^{n}}=\widetilde{\Phi}^{-}(1)\left[N^{*} W_{0} \cap \operatorname{graph} C \Phi^{-}(1)\right]=(0), \\
& \widetilde{\Phi}^{+}(1) N^{*} W_{k} \cap N^{*} \Delta_{\mathbb{R}^{n}}=\widetilde{\Phi}^{+}(1)\left[N^{*} W_{k} \cap \operatorname{graph} C \Phi^{+}(1)\right]=(0),
\end{aligned}
$$

so the transversality hypotheses of Theorem 5.9 are fulfilled. By this theorem, the operator $\bar{\partial}_{A}=G^{-1} \circ \bar{\partial}_{\tilde{A}^{\circ}}^{\circ} F$ is Fredholm of index

(5-48) ind $\bar{\partial}_{A}=\operatorname{ind} \bar{\partial}_{\tilde{A}}=\mu\left(\widetilde{\Phi}^{-} N^{*} W_{0}, N^{*} \Delta_{\mathbb{R}^{n}}\right)-\mu\left(\widetilde{\Phi}^{+} N^{*} W_{k}, N^{*} \Delta_{\mathbb{R}^{n}}\right)$

$$
-\frac{1}{2} \sum_{j=1}^{k}\left(\operatorname{dim} W_{j-1}+\operatorname{dim} W_{j}-2 \operatorname{dim} W_{j-1} \cap W_{j}\right) \text {. }
$$


The symplectic paths $t \mapsto \Phi^{ \pm}(1) \Phi^{ \pm}(1-t / 2)^{-1} \Phi^{ \pm}(t / 2)$ and $t \mapsto \Phi^{ \pm}(t)$ are homotopic by means of the symplectic homotopy

$$
(\lambda, t) \mapsto \Phi^{ \pm}(1) \Phi^{ \pm}\left(\frac{1+\lambda}{2}-\frac{1-\lambda}{2} t\right)^{-1} \Phi^{ \pm}\left(\frac{1+\lambda}{2} t\right),
$$

which fixes the end-points $I$ and $\Phi^{ \pm}(1)$. By the symplectic invariance and the homotopy invariance of the Maslov index we deduce from (5-47) that

$$
\begin{aligned}
\mu\left(\tilde{\Phi}^{-} N^{*} W_{0}, N^{*} \Delta_{\mathbb{R}^{n}}\right) & =\mu\left(N^{*} W_{0}, \tilde{\Phi}^{-}(\cdot)^{-1} N^{*} \Delta_{\mathbb{R}^{n}}\right) \\
& =\mu\left(N^{*} W_{0}, \operatorname{graph} C \Phi^{-}(1) \Phi^{-}(1-\cdot / 2)^{-1} \Phi^{-}(\cdot / 2)\right) \\
& =\mu\left(N^{*} W_{0}, \operatorname{graph} C \Phi^{-}\right)=\mu\left(\operatorname{graph} \Phi^{-} C, N^{*} W_{0}\right)
\end{aligned}
$$

where the lest equality is obtained by applying the antisymplectic involution $C$ to both arguments. Similarly,

$$
\mu\left(\widetilde{\Phi}^{+} N^{*} W_{k}, N^{*} \Delta_{\mathbb{R}^{n}}\right)=\mu\left(\operatorname{graph} \Phi^{+} C, N^{*} W_{k}\right)
$$

The conclusion follows from (5-48), (5-49), and (5-50).

In the case of the right half-strip $\Sigma^{+}$, we fix an integer $k \geq 0$, real numbers

$$
0=s_{0}<s_{1}<\cdots<s_{k}<s_{k+1}=+\infty,
$$

a linear subspace $V_{0} \subset \mathbb{R}^{n}$ and a $(k+1)$-tuple ${ }^{W}=\left(W_{0}, \ldots, W_{k}\right)$ of linear subspaces of $\mathbb{R}^{n} \times \mathbb{R}^{n}$, such that $W_{0}$ and $V_{0} \times V_{0}$ are partially orthogonal, and so are $W_{j-1}$ and $W_{j}$, for every $j=1, \ldots, k$. Set $\mathscr{S}=\left\{s_{1}, \ldots, s_{k}, s_{1}+i, \ldots, s_{k}+i\right\}$, and let $X_{\mathscr{S}, V_{0}, \mathscr{W}}^{1, p}\left(\Sigma^{+}, \mathbb{C}^{n}\right)$ be the completion of the space of maps $u \in C_{\mathscr{Y}, c}^{\infty}\left(\Sigma^{+}, \mathbb{C}^{n}\right)$ such that

$u(i t) \in V_{0} \forall t \in[0,1], \quad(u(s), \bar{u}(s+i)) \in N^{*} W_{j}, \quad \forall s \in\left[s_{j}, s_{j+1}\right], \quad j=0, \ldots, k$,

with respect to the norm $\|u\|_{X^{1, p}\left(\Sigma^{+}\right)}$.

Let $A \in C^{0}\left([0,+\infty] \times[0,1], \mathrm{L}\left(\mathbb{R}^{2 n}, \mathbb{R}^{2 n}\right)\right)$ be such that $A(+\infty, t) \in \operatorname{Sym}(2 n, \mathbb{R})$ for every $t \in[0,1]$, and let $\Phi^{+}:[0,1] \rightarrow \operatorname{Sp}(2 n)$ be the solution of the linear Hamiltonian systems

Then we have:

$$
\frac{d}{d t} \Phi^{+}(t)=i A(+\infty, t) \Phi^{+}(t), \quad \Phi^{+}(0)=I
$$

Theorem 5.24 Assume that graph $C \Phi^{+}(1) \cap N^{*} W_{k}=(0)$. Then for every $p \in$ ] $1,+\infty[$ the $\mathbb{R}$-linear operator

$$
\bar{\partial}_{A}: X_{\mathscr{S}, V_{0}, W}^{1, p}\left(\Sigma^{+}, \mathbb{C}^{n}\right) \rightarrow X_{\mathscr{S}}^{p}\left(\Sigma^{+}, \mathbb{C}^{n}\right), \quad u \mapsto \bar{\partial} u+A u
$$


is bounded and Fredholm of index

$$
\begin{array}{r}
\operatorname{ind} \bar{\partial}_{A}=\frac{n}{2}-\mu\left(\operatorname{graph} \Phi^{+} C, N^{*} W_{k}\right)-\frac{1}{2}\left(\operatorname{dim} W_{0}+2 \operatorname{dim} V_{0}-2 \operatorname{dim} W_{0} \cap\left(V_{0} \times V_{0}\right)\right) \\
-\frac{1}{2} \sum_{j=1}^{k}\left(\operatorname{dim} W_{j-1}+\operatorname{dim} W_{j}-2 \operatorname{dim} W_{j-1} \cap W_{j}\right) .
\end{array}
$$

Proof By the same argument used in the proof of Theorem 5.23, the operator $\bar{\partial}_{A}$ is Fredholm and has the same index as the operator

$$
\bar{\partial}_{\tilde{A}}: X_{\mathscr{S}^{\prime}, V_{0} \times V_{0}, W_{, W^{\prime}}}^{1, p}\left(\Sigma^{+}, \mathbb{C}^{2 n}\right) \rightarrow X_{\mathscr{S}^{\prime}}^{p}\left(\Sigma^{+}, \mathbb{C}^{2 n}\right), \quad u \mapsto \bar{\partial} u+\tilde{A} u,
$$

where $\mathscr{G}^{\prime}=\left\{2 s_{1}, \ldots, 2 s_{k}, 2 s_{1}+i, \ldots, 2 s_{k}+i\right\}, \mathcal{W}^{\prime}$ is the $(k+1)$-tuple $\left(\Delta_{\mathbb{R}^{n}}, \ldots, \Delta_{\mathbb{R}^{n}}\right)$, and

$$
\tilde{A}(z):=\frac{1}{2}(A(z / 2) \oplus C A(\bar{z} / 2+i) C) .
$$

By Theorem 5.21 and by (5-50), the index of this operator is

$$
\begin{aligned}
\text { ind } \bar{\partial} \tilde{A}= & n-\mu\left(\operatorname{graph} \Phi^{+} C, N^{*} W_{k}\right) \\
& -\frac{1}{2}\left(\operatorname{dim} \Delta_{\mathbb{R}^{n}}+\operatorname{dim} V_{0} \times V_{0}-2 \operatorname{dim} \Delta_{\mathbb{R}^{n}} \cap\left(V_{0} \times V_{0}\right)\right) \\
& -\frac{1}{2}\left(\operatorname{dim} W_{0}+\operatorname{dim} V_{0} \times V_{0}-2 \operatorname{dim} W_{0} \cap\left(V_{0} \times V_{0}\right)\right) \\
& -\frac{1}{2} \sum_{j=1}^{k}\left(\operatorname{dim} W_{j-1}+\operatorname{dim} W_{j}-2 \operatorname{dim} W_{j-1} \cap W_{j}\right) \\
= & n-\mu\left(\operatorname{graph} \Phi^{+} C, N^{*} W_{k}\right)-\frac{n}{2} \\
& -\frac{1}{2}\left(\operatorname{dim} W_{0}+2 \operatorname{dim} V_{0}-2 \operatorname{dim} W_{0} \cap\left(V_{0} \times V_{0}\right)\right) \\
& -\frac{1}{2} \sum_{j=1}^{k}\left(\operatorname{dim} W_{j-1}+\operatorname{dim} W_{j}-2 \operatorname{dim} W_{j-1} \cap W_{j}\right) .
\end{aligned}
$$

The desired formula follows.

In the case of the left half-strip $\Sigma^{-}$, let $k, V_{0}, \mathcal{W}$ be as above, and let $\mathscr{Y}=\left\{s_{1}, \ldots, s_{k}\right.$, $\left.s_{1}+i, \ldots, s_{k}+i\right\}$ with

$$
0=s_{0}>s_{1}>\cdots>s_{k}>s_{k+1}=-\infty
$$


Let $X_{\mathscr{Y}, V_{0}, \mathscr{W}}^{1, p}\left(\Sigma^{-}, \mathbb{C}^{n}\right)$ the completion of the space of all maps $u \in C_{\mathscr{S}_{, c}}^{\infty}\left(\Sigma^{-}, \mathbb{C}^{n}\right)$ such that

$u(i t) \in V_{0}, \quad \forall t \in[0,1], \quad(u(s), \bar{u}(s+i)) \in N^{*} W_{j}, \quad \forall s \in\left[s_{j+1}, s_{j}\right], \quad j=0, \ldots, k$, with respect to the norm $\|u\|_{X^{1, p}\left(\Sigma^{-}\right)}$.

Let $A \in C^{0}\left([-\infty, 0] \times[0,1], \mathrm{L}\left(\mathbb{R}^{2 n}, \mathbb{R}^{2 n}\right)\right)$ be such that $A(-\infty, t)$ is symmetric for every $t \in[0,1]$, and let $\Phi^{-}:[0,1] \rightarrow \mathrm{Sp}(2 n)$ be the solution of the linear Hamiltonian systems

$$
\frac{d}{d t} \Phi^{-}(t)=i A(-\infty, t) \Phi^{+}(t), \quad \Phi^{-}(0)=I .
$$

Then we have:

Theorem 5.25 Assume that graph $C \Phi^{-}(1) \cap N^{*} W_{k}=(0)$. Then for every $p \in$ ] $1,+\infty[$ the $\mathbb{R}$-linear operator

$$
\bar{\partial}_{A}: X_{\mathscr{S}, V_{0}, \mathscr{W}}^{1, p}\left(\Sigma^{-}, \mathbb{C}^{n}\right) \rightarrow X_{\mathscr{\varphi}}^{p}\left(\Sigma^{-}, \mathbb{C}^{n}\right), \quad u \mapsto \bar{\partial} u+A u,
$$

is bounded and Fredholm of index

$$
\begin{aligned}
\text { ind } \bar{\partial}_{A}= & \frac{n}{2}+\mu\left(\operatorname{graph} \Phi^{-} C, N^{*} W_{k}\right) \\
& -\frac{1}{2}\left(\operatorname{dim} W_{0}+2 \operatorname{dim} V_{0}-2 \operatorname{dim} W_{0} \cap\left(V_{0} \times V_{0}\right)\right) \\
& -\frac{1}{2} \sum_{j=1}^{k}\left(\operatorname{dim} W_{j-1}+\operatorname{dim} W_{j}-2 \operatorname{dim} W_{j-1} \cap W_{j}\right) .
\end{aligned}
$$

\subsection{Coherent orientations}

As noticed in [4, Section 1.4], the problem of giving coherent orientations for the spaces of maps arising in Floer homology on cotangent bundles is somehow simpler than in the case of a general symplectic manifolds, treated in [25] for periodic orbits and in [29] for more general Lagrangian boundary conditions. This fact remains true if we deal with Cauchy-Riemann type operators on strips and half-strips with jumping conormal boundary conditions. We briefly discuss this issue in the general case of nonlocal boundary conditions on the strip, the case of the half-strip being similar (see [4, Section 3.2]).

We recall that the space Fred $(E, F)$ of Fredholm linear operators from the real Banach space $E$ to the real Banach space $F$ is the base space of a smooth real nontrivial line bundle $\operatorname{det}(\operatorname{Fred}(E, F))$, with fibers

$$
\operatorname{det}(A):=\Lambda^{\max }(\operatorname{ker} A) \otimes\left(\Lambda^{\max }(\operatorname{coker} A)\right)^{*}, \quad \forall A \in \operatorname{Fred}(E, F),
$$


where $\Lambda^{\max }(V)$ denotes the component of top degree in the exterior algebra of the finite-dimensional vector space $V$ (see Quillen [40]).

Let us recall the setting from Section 5.8. We fix the data $k \geq 0, \mathscr{S}=\left\{s_{1}, \ldots, s_{k}\right.$, $\left.s_{1}+i, \ldots, s_{k}+i\right\}$, with $s_{1}<\cdots<s_{k}$, and $\mathcal{W}=\left(W_{0}, \ldots, W_{k}\right)$, where $W_{0}, \ldots, W_{k}$ are linear subspaces of $\mathbb{R}^{n} \times \mathbb{R}^{n}$, such that $W_{j-1}$ is partially orthogonal to $W_{j}$, for $j=1, \ldots, k$. Let $A^{ \pm}:[0,1] \rightarrow \operatorname{Sym}\left(\mathbb{R}^{n}\right)$ be continuous paths of symmetric matrices such that the linear problems

$$
\left\{\begin{array} { l } 
{ w ^ { \prime } ( t ) = i A ^ { - } ( t ) w ( t ) , } \\
{ ( w ( 0 ) , C w ( 1 ) ) \in N ^ { * } W _ { 0 } , }
\end{array} \quad \left\{\begin{array}{l}
w^{\prime}(t)=i A^{+}(t) w(t), \\
(w(0), C w(1)) \in N^{*} W_{k},
\end{array}\right.\right.
$$

have only the trivial solution $w=0$. Such paths are referred to as nondegenerate paths (with respect to $W_{0}$ and $W_{k}$, respectively). Fix some $p>1$, and let $\mathscr{D}_{\mathscr{G}, W}\left(A^{-}, A^{+}\right)$ be the space of operators of the form

$$
\bar{\partial}_{A}: X_{\mathscr{S}, \mathscr{W}}^{1, p}\left(\Sigma, \mathbb{C}^{n}\right) \rightarrow X_{\mathscr{S}}^{p}\left(\Sigma, \mathbb{C}^{n}\right), \quad u \mapsto \bar{\partial} u+A u,
$$

where $A \in C^{0}\left(\overline{\mathbb{R}} \times[0,1], \mathrm{L}\left(\mathbb{R}^{2 n}, \mathbb{R}^{2 n}\right)\right)$ is such that $A( \pm \infty, t)=A^{ \pm}(t)$ for every $t \in[0,1]$. By Theorem 5.23,

$$
\mathscr{D}_{\mathscr{S}, \mathscr{W}}\left(A^{-}, A^{+}\right) \subset \operatorname{Fred}\left(X_{\mathscr{Y}, \mathscr{W}}^{1, p}\left(\Sigma, \mathbb{C}^{n}\right), X_{\mathscr{\varphi}}^{p}\left(\Sigma, \mathbb{C}^{n}\right)\right) .
$$

It is actually a convex subset, therefore the restriction of the determinant bundle to $\mathscr{D}_{\mathscr{S}, W}\left(A^{-}, A^{+}\right)$- that we denote by $\operatorname{det}\left(\mathscr{D}_{\mathscr{S}, W}\left(A^{-}, A^{+}\right)\right)-$is trivial.

Let $\mathcal{S}$ be the family of all subsets of $\Sigma$ consisting of exactly $k$ pairs of opposite boundary points. It is a $k$-dimensional manifold, diffeomorphic to an open subsets of $\mathbb{R}^{k}$. An orientation of $\operatorname{det}\left(\mathscr{D}_{\mathscr{S}, \mathscr{W}}\left(A^{-}, A^{+}\right)\right)$for a given $\mathscr{S}$ in $\mathcal{S}$ uniquely determines an orientation for all choices of $\mathscr{S}^{\prime} \in \mathcal{S}$. Indeed, the disjoint unions

$$
\bigsqcup_{\mathscr{S} \in \mathcal{S}} X_{\mathscr{S}, \mathscr{W}}^{1, p}\left(\Sigma, \mathbb{C}^{n}\right), \quad \bigsqcup_{\mathscr{S} \in \mathcal{S}} X_{\mathscr{S}}^{p}\left(\Sigma, \mathbb{C}^{n}\right),
$$

define locally trivial Banach bundles over $\mathcal{S}$, and the operators $\bar{\partial}_{A}$ define a Fredholm bundle-morphism between them. Since $\mathcal{S}$ is connected and simply connected, an orientation of the determinant space of this operator between the fibers of a given point $\mathscr{S}$ induces an orientation of the determinant spaces of the operators over each $\mathscr{S}^{\prime} \in \mathcal{S}$.

The space of all Fredholm bundle-morphisms between the above Banach bundles induced by operators of the form $\bar{\partial}_{A}$ with fixed asymptotic paths $A^{-}$and $A^{+}$is denoted by $\mathscr{D}_{\mathscr{W}}\left(A^{-}, A^{+}\right)$. An orientation of the determinant bundle over this space of Fredholm bundle-morphisms is denoted by $o_{W}\left(A^{-}, A^{+}\right)$. 
Let $\mathscr{W}=\left(W_{0}, \ldots, W_{k}\right), \mathscr{W}^{\prime}=\left(W_{k}, \ldots, W_{k+k^{\prime}}\right)$ be vectors consisting of consecutively partially orthogonal linear subspaces of $\mathbb{R}^{n} \times \mathbb{R}^{n}$, and set

$$
\mathscr{W} \# W^{\prime}:=\left(W_{0}, \ldots, W_{k+k^{\prime}}\right) .
$$

Let $A_{0}, A_{1}, A_{2}$ be nondegenerate paths with respect to $W_{0}, W_{k}$, and $W_{k+k^{\prime}}$, respectively. Then orientations $o_{W}\left(A_{0}, A_{1}\right)$ and $o_{W^{\prime}}\left(A_{1}, A_{2}\right)$ of $\operatorname{det}\left(\mathscr{D}_{W}\left(A_{0}, A_{1}\right)\right)$ and $\operatorname{det}\left(\mathscr{D}_{W^{\prime}}\left(A_{1}, A_{2}\right)\right)$, respectively, determine in a canonical way a glued orientation

$$
o_{W}\left(A_{0}, A_{1}\right) \# o_{W^{\prime}}\left(A_{1}, A_{2}\right)
$$

of $\operatorname{det}\left(\mathscr{D}_{\mathscr{W} \mathbb{W}^{\prime}}\left(A_{0}, A_{2}\right)\right)$. The construction is analogous to the one described in [25, Section 3]. This way of gluing orientations is associative. A coherent orientation is a set of orientations $o_{W}\left(A^{-}, A^{+}\right)$for each choice of compatible data such that

$$
o_{W \# W^{\prime}}\left(A_{0}, A_{1}\right)=o_{W}\left(A_{0}, A_{1}\right) \# o_{W^{\prime}}\left(A_{1}, A_{2}\right),
$$

whenever the latter glued orientation is well-defined. The proof of the existence of a coherent orientation is analogous to the proof of Theorem 12 in [25].

The choice of such a coherent orientation in this linear setting determines orientations for all the nonlinear objects we are interested in, and such orientations are compatible with gluing. As mentioned above, the fact that we are dealing with the cotangent bundle of an oriented manifold makes the step from the linear setting to the nonlinear one easier. The reason is that we can fix once for all special symplectic trivializations of the bundle $x^{*}\left(T T^{*} M\right)$, for every solution $x$ of our Hamiltonian problem. In fact, one starts by fixing an orthogonal and orientation preserving trivialization of $(\pi \circ x)^{*}(T M)$, and then considers the induced unitary trivialization of $x^{*}\left(T T^{*} M\right)$. Let $u$ be an element in some space $M(x, y)$, consisting of the solutions of a Floer equation on the strip $\Sigma$ which are asymptotic to two Hamiltonian orbits $x$ and $y$ and satisfy suitable jumping conormal boundary conditions. Then we can find a unitary trivialization of $u^{*}\left(T T^{*} M\right)$ which converges to the given unitary trivializations of $x^{*}\left(T T^{*} M\right)$ and $y^{*}\left(T T^{*} M\right)$. We may use such a trivialization to linearize the problem, producing a Fredholm operator in $\mathscr{D}_{\mathscr{S}, W}\left(A^{-}, A^{+}\right)$. Here $A^{-}, A^{+}$are determined by the fixed unitary trivializations of $x^{*}\left(T T^{*} M\right)$ and $y^{*}\left(T T^{*} M\right)$. The orientation of the determinant bundle over $\mathscr{D}_{\mathscr{S}, \mathscr{W}}\left(A^{-}, A^{+}\right)$then induces an orientation of the tangent space of $\mathcal{M}(x, y)$ at $u$, that is an orientation of $M(x, y)$. See [4, Section 1.4] for more details.

When the manifold $M$ is not orientable, one cannot fix once for all trivializations along the Hamiltonian orbits, and the construction of coherent orientations requires understanding the effect of changing the trivialization, as in [25, Lemma 15]. The Floer complex and the pair-of-pants product are still well-defined over integer coefficients, whereas the Chas-Sullivan loop product requires $\mathbb{Z}_{2}$ coefficients. 


\subsection{Nonlinear consequences}

Let us derive the nonlinear consequences of Theorems 5.23, 5.24 and 5.25. Let $Q$ be a finite dimensional Riemannian manifold, and let $R_{0}, \ldots, R_{k}$ be submanifolds of $Q \times Q$, such that $R_{j-1}$ is partially orthogonal to $R_{j}$, for every $j=1, \ldots, k$ (with respect to the product metric on $Q \times Q$ ). Partial orthogonality implies that the dimension of $R_{j-1} \cap R_{j}$ is locally constant, and we assume such a dimension is actually constant. Let $k \geq 0$ be an integer, and let us fix numbers

$$
-\infty=s_{0}<s_{1}<\cdots<s_{k}<s_{k+1}=+\infty \text {. }
$$

We recall from Section 3.1 that if $H \in C^{\infty}\left([0,1], T^{*} Q\right)$ is a Hamiltonian, the symbol $\mathscr{P}^{R}(H)$ denotes the set of all the Hamiltonian orbits $x:[0,1] \rightarrow T^{*} Q$ which satisfy the boundary condition $(x(0), \mathscr{C} x(1)) \in N^{*} R$, where $\mathscr{C}$ is the antisymplectic involution on $T^{*} Q$ which maps $(q, p)$ into $(q,-p)$. When $x \in \mathscr{P}^{R}(H)$ is nondegenerate, its Maslov index $\mu^{R}(x)$ is defined in (3-5).

We recall that a space $\mathcal{M}$ is said to have virtual dimension $d$, where $d \in \mathbb{Z}$, or briefly

$$
\operatorname{virdim} \mu=d,
$$

if $M$ can be seen as the set of zeroes of a smooth section of some Banach bundle, whose fiberwise derivative is Fredholm of index $d$. When such a section is transverse to the zero-section, the implicit function theorem implies that either $\mathcal{M}$ is empty, or $\mathcal{M}$ is a smooth manifold of dimension $d \geq 0$.

We start by considering the case of the full strip $\Sigma=\{z \in \mathbb{C} \mid 0 \leq \operatorname{Im} z \leq 1\}$ :

Corollary 5.26 Let $x \in \mathscr{P}^{R_{0}}(H)$ and $y \in \mathscr{P}^{R_{k}}(H)$ be nondegenerate. Then the set $M(x, y)$ of maps $u: \Sigma \rightarrow T^{*} Q$ which solve the equation $\bar{\partial}_{J, H}(u)=0$ with boundary conditions

$$
(u(s), \mathscr{C} u(s+i)) \in N^{*} R_{j}, \quad \forall s \in\left[s_{j}, s_{j+1}\right],
$$

and asymptotic conditions

$$
\lim _{s \rightarrow-\infty} u(s+i t)=x(t), \quad \lim _{s \rightarrow+\infty} u(s+i t)=y(t),
$$

has virtual dimension

$$
\operatorname{virdim} M(x, y)=\mu^{R_{0}}(x)-\mu^{R_{k}}(y)-\sum_{j=1}^{k}\left(\operatorname{dim} R_{j-1}-\operatorname{dim} R_{j-1} \cap R_{j}\right) .
$$


Proof Standard arguments in Floer theory allow us to see $M(x, y)$ as the set of zeroes of a smooth section of a suitable Banach bundle, the base of which is a Banach manifold modeled on $X_{\mathscr{S}, \mathscr{W}}^{1, p}$, and the fibers are Banach spaces of sections of class $X_{\mathscr{Y}}^{p}$, where $p>2$. The fiberwise derivative of such a section at $u \in M(x, y)$ is conjugated to a linear operator $\bar{\partial}_{A}$ of the form considered in Theorem 5.23, where the linear subspaces $W_{j}$ of $\mathbb{R}^{n} \times \mathbb{R}^{n}$ are local models for the submanifolds $R_{j}$, and where

$$
\begin{aligned}
& \mu\left(\operatorname{graph} \Phi^{-} C, N^{*} W_{0}\right)=\mu^{R_{0}}(x)-\frac{1}{2}\left(\operatorname{dim} R_{0}-\operatorname{dim} Q\right), \\
& \mu\left(\operatorname{graph} \Phi^{+} C, N^{*} W_{k}\right)=\mu^{R_{k}}(y)-\frac{1}{2}\left(\operatorname{dim} R_{k}-\operatorname{dim} Q\right),
\end{aligned}
$$

by the definition (3-5) of the Maslov index. By Theorem 5.23, such an operator is Fredholm of index

$$
\begin{aligned}
\text { ind } \bar{\partial}_{A}=\mu^{R_{0}}(x)-\frac{1}{2}\left(\operatorname{dim} R_{0}-\operatorname{dim} Q\right)-\mu^{R_{k}}(y)+\frac{1}{2}\left(\operatorname{dim} R_{k}-\operatorname{dim} Q\right) \\
\qquad-\frac{1}{2} \sum_{j=1}^{k}\left(\operatorname{dim} R_{j-1}+\operatorname{dim} R_{j}-2 \operatorname{dim} R_{j-1} \cap R_{j}\right) .
\end{aligned}
$$

After simplification, this formula reduces to

$$
\text { ind } \bar{\partial}_{A}=\mu^{R_{0}}(x)-\mu^{R_{k}}(y)-\sum_{j=1}^{k}\left(\operatorname{dim} R_{j-1}-\operatorname{dim} R_{j-1} \cap R_{j}\right),
$$

as claimed.

Remark 5.27 By elliptic regularity, the maps $u \in M(x, y)$ are smooth up to the boundary on $\Sigma \backslash\left\{s_{1}, s_{1}+i, \ldots, s_{k}, s_{k}+i\right\}$. By Schwarz reflection, the maps

$$
\zeta \mapsto u\left(s_{j}+\zeta^{2}\right) \quad \text { and } \quad \zeta \mapsto u\left(s_{j}+i-\zeta^{2}\right)
$$

are smooth up to the boundary in a neighborhood of zero in the upper right quadrant $\mathrm{Cl}\left(\mathbb{H}^{+}\right)=\{\zeta \in \mathbb{C} \mid \operatorname{Re} \zeta \geq 0, \operatorname{Im} z \geq 0\}$. Analogous regularity results hold for the maps which appear in the following two corollaries.

Remark 5.28 In this paper, all the pairs of submanifolds we need to consider are partially orthogonal. However, it might be useful to have a generalization of Corollary 5.26 to the situation where the submanifolds $R_{j-1}$ and $R_{j}$ are only assumed to have a clean intersection. An easy way to deal with such a situation is the following. First, we transform the nonlocal boundary problem for $u: \Sigma \rightarrow T^{*} Q$ into a local one, by considering the maps $v: \Sigma \rightarrow T^{*}(Q \times Q)$ defined by

$$
v(z):=(\mathscr{C u}((i-z) / 2), u((i+z) / 2)) .
$$


Then $v$ solves a Cauchy-Riemann type equation, together with local boundary conditions

$$
v(s) \in N^{*} \Delta_{Q}, \forall s \in \mathbb{R}, \quad v(s+i) \in N^{*} R_{j}, \forall s \in\left[s_{j}, s_{j+1}\right] .
$$

Let $g_{s}$ be a smooth 1-parameter family of metrics on $Q \times Q$ such that $R_{j-1}$ is partially orthogonal to $R_{j}$ with respect to the metric $g_{s_{j}}$ (when the sets $R_{j-1} \cap R_{j}$ are pairwise disjoint, the family $g_{s}$ can be chosen to be independent of $s$ ). Then the analogue of Corollary 5.26 holds, where the perturbed Cauchy-Riemann operator $\bar{\partial}_{J, H}$ is the one associated to the $s$-dependent family of Levi-Civita almost complex structures $J_{s}$ on $T^{*}(Q \times Q)$ induced by $g_{s}$. The same considerations apply to the next two corollaries.

In the case of the right half-strip $\Sigma^{+}=\{z \in \mathbb{C} \mid \operatorname{Re} z \geq 0,0 \leq \operatorname{Im} z \leq 1\}$, we fix the numbers

$$
0=s_{0}<s_{1}<\cdots<s_{k}<s_{k+1}=+\infty,
$$

and we have the following consequence of Theorem 5.24:

Corollary 5.29 Let $x \in \mathscr{P}^{R_{k}}(H)$ be nondegenerate and let $\gamma \in W^{1,2}([0,1], Q)$ be a curve such that $(\gamma(0), \gamma(1)) \in R_{0}$. Then the set $\mu(\gamma, x)$ of maps $u: \Sigma^{+} \rightarrow T^{*} Q$ which solve the equation $\bar{\partial}_{J, H}(u)=0$ with boundary conditions

$$
(u(s), \mathscr{b} u(s+i)) \in N^{*} R_{j}, \forall s \in\left[s_{j}, s_{j+1}\right], \quad u(i t) \in T_{\gamma(t)}^{*} Q, \forall t \in[0,1],
$$

and the asymptotic condition

$$
\lim _{s \rightarrow+\infty} u(s+i t)=x(t)
$$

has virtual dimension

$$
\operatorname{virdim} \mu(\gamma, x)=-\mu^{R_{k}}(x)-\sum_{j=1}^{k}\left(\operatorname{dim} R_{j-1}-\operatorname{dim} R_{j-1} \cap R_{j}\right) .
$$

Proof Arguing as in the proof of Corollary 5.26, we find that $M(\gamma, x)$ has virtual dimension equal to the Fredholm index of an operator $\bar{\partial}_{A}$ of the form considered in Theorem 5.24, with $V_{0}=(0)$ and $W_{j}$ a local model for $R_{j}$ (see [4, Section 3.1] for more details on how to deal with this kind of boundary data). 
By Theorem 5.24 and (3-5), we have

$$
\text { ind } \begin{aligned}
\bar{\partial}_{A}= & \frac{1}{2} \operatorname{dim} Q-\mu\left(\operatorname{graph} \Phi^{+} C, N^{*} W_{k}\right)-\frac{1}{2} \operatorname{dim} R_{0} \\
& -\frac{1}{2} \sum_{j=1}^{k}\left(\operatorname{dim} R_{j-1}+\operatorname{dim} R_{j}-2 \operatorname{dim} R_{j-1} \cap R_{j}\right) \\
= & -\mu^{R_{k}}(x)-\sum_{j=1}^{k}\left(\operatorname{dim} R_{j-1}-\operatorname{dim} R_{j-1} \cap R_{j}\right),
\end{aligned}
$$

concluding the proof.

In the case of the left half-strip $\Sigma^{-}=\{z \in \mathbb{C} \mid \operatorname{Re} z \leq 0,0 \leq \operatorname{Im} z \leq 1\}$, we fix the numbers

$$
0=s_{0}>s_{1}>\cdots>s_{k}>s_{k+1}=-\infty .
$$

One could easily derive the analogue of Corollary 5.29 from Theorem 5.25. Instead, we prefer to derive the following variant, where $\mathbb{O}_{Q}$ denotes image of the zero-section in $T^{*} Q$ :

Corollary 5.30 Let $x \in \mathscr{P}^{R_{k}}(H)$ be nondegenerate. Then the set $\mathcal{M}(x)$ of maps $u: \Sigma^{-} \rightarrow T^{*} Q$ which solve the equation $\bar{\partial}_{J, H}(u)=0$ with boundary conditions

$$
(u(s), \mathscr{C} u(s+i)) \in N^{*} R_{j}, \quad \forall s \in\left[s_{j+1}, s_{j}\right], \quad u(i t) \in \mathbb{O}_{Q}, \quad \forall t \in[0,1],
$$

and the asymptotic condition

$$
\lim _{s \rightarrow-\infty} u(s+i t)=x(t)
$$

has virtual dimension

$$
\operatorname{virdim} M(x)=\mu^{R_{k}}(x)-\sum_{j=1}^{k}\left(\operatorname{dim} R_{j}-\operatorname{dim} R_{j-1} \cap R_{j}\right) .
$$

Proof Arguing as in the proof of Corollary 5.26, we find that $M(x)$ has virtual dimension equal to the Fredholm index of an operator $\bar{\partial}_{A}$ of the form considered in Theorem 5.25, with $V_{0}=\mathbb{R}^{m}, m=\operatorname{dim} Q$, and $W_{j}$ a local model for $R_{j}$. By 
Theorem 5.25 and (3-5), we have

$$
\text { ind } \begin{aligned}
\bar{\partial}_{A}= & \frac{1}{2} \operatorname{dim} Q+\mu\left(\operatorname{graph} \Phi^{-} C, N^{*} W_{k}\right)-\frac{1}{2}\left(2 \operatorname{dim} Q-\operatorname{dim} R_{0}\right) \\
& -\frac{1}{2} \sum_{j=1}^{k}\left(\operatorname{dim} R_{j-1}+\operatorname{dim} R_{j}-2 \operatorname{dim} R_{j-1} \cap R_{j}\right) \\
= & \mu^{R_{k}}(x)-\sum_{j=1}^{k}\left(\operatorname{dim} R_{j}-\operatorname{dim} R_{j-1} \cap R_{j}\right),
\end{aligned}
$$

concluding the proof.

Dimension computations We conclude this section by using the above corollaries to prove that all the spaces of solutions of the Cauchy-Riemann type problems considered in this paper are - generically - smooth manifolds, and to compute their dimension. Together with the results of Section 5.9, we deduce that these manifolds carry coherent orientations which are compatible with gluing. The transversality issues which lead to genericity in the space of the Hamiltonians are standard; see Floer, Hofer and Salamon [26]. Here we compute the virtual dimensions, by making use of the following two lemmas.

Lemma 5.31 Let $R_{1}, R_{2}$ be submanifolds of $Q \times Q$, let $H_{1}, H_{2} \in C^{\infty}\left([0,1] \times T^{*} Q\right)$ be two Hamiltonians, and set

$$
\begin{aligned}
& R:=\left\{\left(q_{1}, q_{2}, q_{3}, q_{4}\right) \in Q^{4} \mid\left(q_{3}, q_{1}\right) \in R_{1},\left(q_{2}, q_{4}\right) \in R_{2}\right\} \cong R_{1} \times R_{2}, \\
& K \in C^{\infty}\left([0,1] \times T^{*} Q^{2}\right), \quad K\left(t, x_{1}, x_{2}\right):=H_{1}\left(1-t, \mathscr{b} x_{1}\right)+H_{2}\left(t, x_{2}\right) .
\end{aligned}
$$

Then the curves $x_{1}, x_{2} \in C^{\infty}\left([0,1], T^{*} Q\right)$ belong to $\mathscr{P} R_{1}\left(H_{1}\right)$ and $\mathscr{P} R_{2}\left(H_{2}\right)$, respectively, if and only if the curve

$$
x:[0,1] \rightarrow T^{*}(Q \times Q)=T^{*} Q \times T^{*} Q, \quad x(t):=\left(\mathscr{b} x_{1}(1-t), x_{2}(t)\right),
$$

belongs to $\mathscr{P}^{R}(K)$. Furthermore,

$$
\mu^{R}(x)=\mu^{R_{1}}\left(x_{1}\right)+\mu^{R_{2}}\left(x_{2}\right) .
$$

Proof It is easy to check that $x_{1}$ and $x_{2}$ are orbits of the Hamiltonian vector fields associated to $H_{1}$ and $H_{2}$ if and only if $y$ is an orbit of the vector field associated to $K$. Moreover,

$$
(y(0), \mathscr{b} y(1))=\left(\mathscr{b} x_{1}(1), x_{2}(0), x_{1}(0), \mathscr{b} x_{2}(1)\right)
$$


belongs to $N^{*} R$ if and only if $\left(x_{1}(0), \mathscr{C} x_{1}(1)\right)$ belongs to $N^{*} R_{1}$ and $\left(x_{2}(0), \mathscr{C} x_{2}(1)\right)$ belongs to $N^{*} R_{2}$. We just have to check the identity involving the Maslov indices.

Let $j$ be either 1 or 2 . Let $G_{H_{j}}:[0,1] \rightarrow \operatorname{Sp}(2 m), m=\operatorname{dim} Q$, be the symplectic path obtained by conjugating the differential of the Hamiltonian flow of $H_{j}$ along the orbit $x_{j}$ by a vertical-preserving trivialization $\Psi_{j}$ of $x_{j}^{*}\left(T T^{*} Q\right)$ such that

$$
\left(\Psi_{j}(0) \times C \Psi_{j}(1) D^{\mathscr{C}}\left(\mathscr{b} x_{j}(1)\right)\right) T_{\left(x_{j}(0), \mathscr{b} x_{j}(1)\right)} N^{*} R_{j}=N^{*} W_{j},
$$

where $W_{j}$ is a linear subspace of $\mathbb{R}^{m} \times \mathbb{R}^{m}$. Then, by the definition (3-5) of the Maslov index,

$$
\mu^{R_{j}}\left(x_{j}\right)=\mu\left(\operatorname{graph} G_{H_{j}} C, N^{*} W_{j}\right)+\frac{1}{2}\left(\operatorname{dim} R_{j}-\operatorname{dim} Q_{j}\right) .
$$

If $G_{K}:[0,1] \rightarrow \mathrm{Sp}(4 m)$ is the symplectic path obtained by conjugating the differential of the Hamiltonian flow of $K$ along the orbit $y$ by the trivialization induced in the obvious way by $\Psi_{1}$ and $\Psi_{2}$, we have

$$
\begin{aligned}
& G_{K}(t)=C G_{H_{1}}(1-t) G_{H_{1}}(1)^{-1} C \times G_{H_{2}}(t), \\
& \mu^{R}(y)=\mu\left(\operatorname{graph} G_{K} C, N^{*} W\right)+\frac{1}{2}\left(\operatorname{dim} R-\operatorname{dim} Q^{2}\right),
\end{aligned}
$$

where $\quad W:=\left\{\left(\xi_{1}, \xi_{2}, \xi_{3}, \xi_{4}\right) \mid\left(\xi_{3}, \xi_{1}\right) \in W_{1},\left(\xi_{2}, \xi_{4}\right) \in W_{2}\right\}$.

Since $\operatorname{dim} R=\operatorname{dim} Q_{1}+\operatorname{dim} Q_{2}$, we must show that

(5-53) $\mu\left(\right.$ graph $\left.G_{K} C, N^{*} W\right)=\mu\left(\operatorname{graph} G_{H_{1}} C, N^{*} W_{1}\right)+\mu\left(\operatorname{graph} G_{H_{2}} C, N^{*} W_{2}\right)$.

The linear mapping

$$
T: \mathbb{R}^{4 m} \rightarrow \mathbb{R}^{4 m}, \quad\left(\xi_{1}, \xi_{2}, \xi_{3}, \xi_{4}\right) \mapsto\left(\xi_{3}, \xi_{1}, \xi_{2}, \xi_{4}\right),
$$

maps $W$ onto $W_{1} \times W_{2}$, hence the symplectic automorphism $T \oplus T^{*}$ maps $N^{*} W$ onto $N^{*} W_{1} \times N^{*} W_{2}$. Moreover,

$$
\left(T \oplus T^{*}\right) \operatorname{graph} G_{K}(t) C=\left(\operatorname{graph} G_{H_{1}}(1) G_{H_{1}}(1-t)^{-1} C\right) \times\left(\operatorname{graph} G_{H_{2}}(t) C\right) .
$$

By the symplectic invariance and the additivity of the Maslov index,

$$
\begin{aligned}
& \mu\left(\operatorname{graph} G_{K} C, N^{*} W\right)=\mu\left(\left(T \oplus T^{*}\right) \operatorname{graph} G_{K}(t) C,\left(T \oplus T^{*}\right) N^{*} W\right) \\
& \quad=\mu\left(\operatorname{graph} G_{H_{1}}(1) G_{H_{1}}(1-\cdot)^{-1} C, N^{*} W_{1}\right)+\mu\left(\operatorname{graph} G_{H_{2}} C, N^{*} W_{2}\right) .
\end{aligned}
$$

The symplectic path $t \mapsto G_{H_{1}}(1) G_{H_{1}}(1-t)^{-1}$ is homotopic within the symplectic group to the path $G_{H_{1}}$, by the homotopy

$$
(\lambda, t) \mapsto G_{H_{1}}(t+\lambda(1-t)) G_{H_{1}}(\lambda(1-t))^{-1},
$$


which fixes the end points $I$ and $G_{H_{1}}(1)$. By the homotopy invariance of the Maslov index,

$$
\mu\left(\operatorname{graph} G_{H_{1}}(1) G_{H_{1}}(1-\cdot)^{-1} C, N^{*} W_{1}\right)=\mu\left(\operatorname{graph} G_{H_{1}} C, N^{*} W_{1}\right),
$$

so (5-53) follows from (5-54).

Lemma 5.32 Let $R$ be a submanifold of $Q \times Q$, let $H \in C^{\infty}\left([0,1] \times T^{*} Q\right)$, and define $K \in C^{\infty}\left([0,1] \times T^{*} Q^{2}\right)$ by

$$
K\left(t, x_{1}, x_{2}\right):=\frac{1}{2} H\left(\frac{1-t}{2}, \mathscr{b} x_{1}\right)+\frac{1}{2} H\left(\frac{1+t}{2}, x_{2}\right) .
$$

Then the curve $x:[0,1] \rightarrow T^{*} Q$ belongs to $\mathscr{P}^{R}(H)$ if and only if the curve $y:[0,1] \rightarrow$ $T^{*} Q^{2}$, defined by

$$
y(t):=\left(\mathscr{b} x\left(\frac{1-t}{2}\right), x\left(\frac{1+t}{2}\right)\right),
$$

belongs to $\mathscr{P}^{\Delta_{Q} \times R}(K)$. Furthermore,

$$
\mu^{R}(x)=\mu^{\Delta_{Q} \times R}(y) .
$$

Proof A simple computation shows that $x$ is an orbit of the Hamiltonian vector field induced by $H$ if and only if $y$ is an orbit of the one induced by $K$. Moreover,

$$
(y(0), \mathscr{b} y(1))=(\mathscr{b} x(1 / 2), x(1 / 2), \mathscr{b} x(0), x(1)) .
$$

so, by using the fact that conormals are $\mathscr{C}$-invariant, we deduce that $x \in \mathscr{P}^{R}(H)$ if and only if $y \in \mathscr{P}^{\Delta_{Q} \times R}(K)$. Let $G_{H}:[0,1] \rightarrow \operatorname{Sp}(2 m), m=\operatorname{dim} Q$, be the symplectic path obtained by conjugating the differential of the Hamiltonian flow of $H$ along the orbit $x$ by a vertical-preserving trivialization $\Psi$ of $x^{*}\left(T T^{*} Q\right)$ such that

$$
\left(\Psi(0) \times C \Psi(1) D_{\mathscr{C}}(\mathscr{C} x(1))\right) T_{(x(0), \mathscr{b} x(1))} N^{*} R=N^{*} W,
$$

where $W$ is a linear subspace of $\mathbb{R}^{m} \times \mathbb{R}^{m}$. Then, by the definition (3-5) of the Maslov index,

$$
\mu^{R}(x)=\mu\left(\operatorname{graph} G_{H} C, N^{*} W\right)+\frac{1}{2}(\operatorname{dim} R-\operatorname{dim} Q) .
$$

If $G_{K}:[0,1] \rightarrow \mathrm{Sp}(4 m)$ is the symplectic path obtained by conjugating the differential of the Hamiltonian flow of $K$ along the orbit $y$ by the trivialization induced in the obvious way by $\Psi$, we have

$$
G_{K}(t)=\left(C G_{H}\left(\frac{1-t}{2}\right) G_{H}\left(\frac{1}{2}\right)^{-1} C\right) \times\left(G_{H}\left(\frac{1+t}{2}\right) G_{H}\left(\frac{1}{2}\right)^{-1}\right) .
$$


Moreover, using also (5-3),

$$
\begin{aligned}
\mu^{\Delta_{Q} \times R}(y) & =\mu\left(\operatorname{graph} G_{K} C, N^{*}\left(\Delta_{\mathbb{R}^{m}} \times W\right)\right)+\frac{1}{2}\left(\operatorname{dim} R \times \Delta_{Q}-\operatorname{dim} Q \times Q\right) \\
& =\mu\left(\operatorname{graph} G_{K} C, N^{*} \Delta_{\mathbb{R}^{m}} \times N^{*} W\right)+\frac{1}{2}(\operatorname{dim} R-\operatorname{dim} Q) \\
& =\mu\left(G_{K} N_{\Delta_{\mathbb{R}}}^{*}, N^{*} W\right)+\frac{1}{2}(\operatorname{dim} R-\operatorname{dim} Q) .
\end{aligned}
$$

Since $N^{*} \Delta_{\mathbb{R}^{m}}=\operatorname{graph} C$, there holds

$$
G_{K}(t) N_{\Delta_{\mathbb{R}} m}^{*}=\operatorname{graph} \Gamma(t) C,
$$

where $\Gamma:[0,1] \rightarrow \operatorname{Sp}(2 m)$ is the path

$$
\Gamma(t):=G_{H}\left(\frac{1+t}{2}\right) G_{H}\left(\frac{1-t}{2}\right)^{-1} .
$$

The symplectic path $\Gamma$ is homotopic to the symplectic path $G_{H}$ by the symplectic homotopy

$$
(\lambda, t) \mapsto G_{H}\left(t+\frac{\lambda}{2}(1-t)\right) G_{H}\left(\frac{\lambda}{2}(1-t)\right)^{-1},
$$

which fixes the end points $\Gamma(0)=G_{H}(0)=I$ and $\Gamma(1)=G_{H}(1)$. By the homotopy invariance of the Maslov index,

$$
\mu\left(G_{K} N_{\Delta_{\mathbb{R}^{m}}}^{*}, N^{*} W\right)=\mu\left(\operatorname{graph} \Gamma C, N^{*} W\right)=\mu\left(\operatorname{graph} G_{H} C, N^{*} W\right),
$$

and the conclusion follows from the above formulas for $\mu^{R}(x)$ and $\mu^{\Delta_{Q} \times R}(y)$.

We are finally ready to compute the virtual dimensions of the spaces of maps introduced in this paper.

The space $\mu_{\Upsilon}^{\Lambda}$ Let us study the space of solutions $\mu_{\Upsilon}^{\Lambda}\left(x_{1}, x_{2} ; y\right)$, where $x_{1} \in$ $\mathscr{P}^{\Lambda}\left(H_{1}\right), x_{2} \in \mathscr{P}^{\Lambda}\left(H_{2}\right)$, and $y \in \mathscr{P}^{\Lambda}\left(H_{1} \# H_{2}\right)$ (see Section 3.3). It is a space of solutions of the Floer equation on the pair-of-pants Riemann surface $\Sigma_{\Upsilon}^{\Lambda}$. The pair-ofpants Riemann surface $\Sigma_{\Upsilon}^{\Lambda}$ is described in Section 3.2 as the quotient of the disjoint union of two strips $\mathbb{R} \cup[-1,0]$ and $\mathbb{R} \times[0,1]$ with respect to the identifications

$$
\begin{aligned}
& (s,-1) \sim(s, 0-), \quad(s, 0+) \sim(s, 1) \\
& \forall s \leq 0, \\
& (s,-1) \sim(s, 1), \quad(s, 0-) \sim(s, 0+) \\
& \forall s \geq 0 \text {. }
\end{aligned}
$$

The space $\mu_{\Upsilon}^{\Lambda}\left(x_{1}, x_{2} ; y\right)$ consists of maps

$$
u: \Sigma_{\Upsilon}^{\Lambda} \rightarrow T^{*} M
$$


solving the Floer equation $\bar{\partial}_{J, H}(u)=0$, with asymptotics

$$
\lim _{s \rightarrow-\infty} u(s, t-1)=x_{1}(t), \quad \lim _{s \rightarrow-\infty} u(s, t)=x_{2}(t), \quad \lim _{s \rightarrow+\infty} u(s, 2 t-1)=y(t) .
$$

We can associate to a map $u: \Sigma_{\Upsilon}^{\Lambda} \rightarrow T^{*} M$ the map $v: \Sigma \rightarrow T^{*} M^{2}$ by setting

$$
v(z):=(\mathscr{b} u(\bar{z}), u(z)) .
$$

The identifications (5-55) on the left-hand side of the domain of $u$ are translated into the fact that $v(s+i t)$ is 1 periodic in $t$ for $s \leq 0$, or equivalently into the nonlocal boundary condition

$$
(v(s), \mathscr{C} v(s+i)) \in N^{*} \Delta_{M^{2}}, \quad \forall s \leq 0,
$$

where $\Delta_{M^{2}}$ denotes the diagonal in $M^{4}=M^{2} \times M^{2}$. The identifications (5-56) on the right-hand side of the domain of $u$ are translated into the local boundary conditions

$$
v(s) \in N^{*} \Delta_{M}, \quad v(s+i) \in N^{*} \Delta_{M}, \quad \forall s \geq 0 .
$$

The map $u$ solves the Floer equation $\bar{\partial}_{J, H}(u)=0$ if and only if $v$ solves the Floer equation $\bar{\partial}_{J, K}(v)=0$, where $K$ is the Hamiltonian defined in (5-52). The asymptotic conditions for $u$ are equivalent to

$$
\begin{aligned}
& x(t):=\lim _{s \rightarrow-\infty} v(s+i t)=\left(\mathscr{b} x_{1}(1-t), x_{2}(t)\right), \\
& z(t):=\lim _{s \rightarrow+\infty} v(s+i t)=(\mathscr{b} y((1-t) / 2), y((1+t) / 2)) .
\end{aligned}
$$

We conclude that $\mu_{\Upsilon}^{\Lambda}\left(x_{1}, x_{2} ; y\right)$ can be identified with the space of maps $\mathcal{M}(x, z)$ of Corollary 5.26, where the underlying manifold is $Q=M \times M$, the Hamiltonian is $K$, the boundary conditions have a single jump at $s_{1}=0$ and are given by the following partially orthogonal submanifolds of $Q^{2}=M^{4}$ :

$$
R_{0}=\Delta_{M^{2}}, \quad R_{1}=\Delta_{M} \times \Delta_{M} .
$$

By Corollary 5.26,

$$
\begin{aligned}
\operatorname{virdim} \mu_{\Upsilon}^{\Lambda} & \left(x_{1}, x_{2} ; y\right) \\
& =\mu^{\Delta_{M^{2}}}(x)-\mu^{\Delta_{M} \times \Delta_{M}}(z)-\left(\operatorname{dim} \Delta_{M^{2}}-\operatorname{dim} \Delta_{M^{2}} \cap\left(\Delta_{M} \times \Delta_{M}\right)\right) \\
& =\mu^{\Delta_{M^{2}}}(x)-\mu^{\Delta_{M} \times \Delta_{M}}(z)-n .
\end{aligned}
$$

By Lemma 5.31,

$$
\mu^{\Delta_{M^{2}}}(x)=\mu^{\Delta_{M}}\left(x_{1}\right)+\mu^{\Delta_{M}}\left(x_{2}\right)=\mu^{\Lambda}\left(x_{1}\right)+\mu^{\Lambda}\left(x_{2}\right) .
$$


By Lemma 5.32, applied to the manifold $Q=M$, to the Hamiltonian $H_{1} \# H_{2}$, and to the submanifold $R=\Delta_{M}$,

$$
\mu^{\Delta_{M} \times \Delta_{M}}(z)=\mu^{\Delta_{M}}(y)=\mu^{\Lambda}(y) .
$$

We conclude that $\mu_{\Upsilon}^{\Lambda}\left(x_{1}, x_{2} ; y\right)$ has virtual dimension

$$
\operatorname{virdim} \mu_{\Upsilon}^{\Lambda}\left(x_{1}, x_{2} ; y\right)=\mu^{\Lambda}\left(x_{1}\right)+\mu^{\Lambda}\left(x_{2}\right)-\mu^{\Lambda}(y)-n
$$

proving the part of Proposition 3.4 which concerns the space $\mu_{\Upsilon}^{\Lambda}$.

The space $\mu_{\Upsilon}^{\Omega}$ Let us consider the space $\mu_{\Upsilon}^{\Omega}\left(x_{1}, x_{2} ; y\right)$, where $x_{1} \in \mathscr{P}^{\Omega}\left(H_{1}\right), x_{2} \in$ $\mathscr{P}^{\Omega}\left(H_{2}\right)$, and $y \in \mathscr{P}^{\Omega}\left(H_{1} \# H_{2}\right)$ (see Section 3.3). This is the space of solutions $u$ of the Floer equation on the Riemann surface with boundary $\Sigma_{\Upsilon}^{\Omega}$, described as a strip with a slit in Section 3.2, which take values in $T_{q_{0}}^{*} M$ on the boundary, and converge to the orbits $x_{1}, x_{2}, y$ on the three ends. By defining the map $v: \Sigma \rightarrow T^{*} M^{2}$ as in (5-57) and the curves $x$ and $z$ as in (5-60), we see that the space $\mu_{\Upsilon}^{\Omega}\left(x_{1}, x_{2} ; y\right)$ is in one-to-one correspondence with the space $M(x, z)$ of Corollary 5.26, where $Q=M^{2}$, the Hamiltonian is the function $K$ defined in (5-52), the boundary conditions jump at $s_{1}=0$ and are given by the following partially orthogonal submanifolds of $Q^{2}=M^{4}$ :

$$
R_{0}=\left\{\left(q_{0}, q_{0}, q_{0}, q_{0}\right)\right\}, \quad R_{1}=\Delta_{M} \times\left\{\left(q_{0}, q_{0}\right)\right\} .
$$

By Corollary 5.26,

$$
\operatorname{virdim} \mu_{\Upsilon}^{\Omega}\left(x_{1}, x_{2} ; y\right)=\mu^{\left(q_{0}, q_{0}, q_{0}, q_{0}\right)}(x)-\mu^{\Delta_{M} \times\left(q_{0}, q_{0}\right)}(z) .
$$

By Lemma 5.31,

$$
\mu^{\left(q_{0}, q_{0}, q_{0}, q_{0}\right)}(x)=\mu^{\left(q_{0}, q_{0}\right)}\left(x_{1}\right)+\mu^{\left(q_{0}, q_{0}\right)}\left(x_{2}\right)=\mu^{\Omega}\left(x_{1}\right)+\mu^{\Omega}\left(x_{2}\right) .
$$

By Lemma 5.32,

$$
\mu^{\Delta_{M} \times\left(q_{0}, q_{0}\right)}(z)=\mu^{\left(q_{0}, q_{0}\right)}(y)=\mu^{\Omega}(y) .
$$

Therefore, $\mu_{\Upsilon}^{\Omega}\left(x_{1}, x_{2} ; y\right)$ has virtual dimension

$$
\operatorname{virdim} \mu_{\Upsilon}^{\Omega}\left(x_{1}, x_{2} ; y\right)=\mu^{\Omega}\left(x_{1}\right)+\mu^{\Omega}\left(x_{2}\right)-\mu^{\Omega}(y) .
$$

This concludes the proof of Proposition 3.4.

The space $\mu_{E}$ Let $\left(x_{1}, x_{2}\right) \in \mathscr{P} \Lambda\left(H_{1}\right) \times \mathscr{P} \Lambda\left(H_{2}\right)$ and $y \in \mathscr{P}^{\Theta}\left(H_{1} \oplus H_{2}\right)$ (see Section 3.4). We set $Q=M \times M$, we define the Hamiltonian $K \in C^{\infty}\left([0,1] \times T^{*} Q\right)$ by (5-52), and the $T^{*} Q$-valued curves $x$ and $z$ by

$$
x(t):=\left(\mathscr{C} x_{1}(1-t), x_{2}(t)\right), \quad z(t):=\left(\mathscr{b} y_{1}(1-t), y_{2}(t)\right),
$$


where $y_{1}$ and $y_{2}$ are the components of $y$. Then $x \in \mathscr{P}^{\Delta_{M}}(K), z \in \mathscr{P}_{M}^{(4)}(K)$, and one easily checks that

$$
\mu^{\Delta_{M}^{(4)}}(z)=\mu^{\Delta_{M}^{(4)}}(y)=\mu^{\Theta}(y),
$$

where the Maslov index of $z$ refers to the Hamiltonian $K$, and the Maslov index of $y$ to the Hamiltonian $H_{1} \oplus H_{2}$. The space of solutions $M_{E}\left(x_{1}, x_{2} ; y\right)$ is in one-toone correspondence with the space $M(x, z)$ of Corollary 5.26, where the boundary conditions switch at $s_{1}=0$ and are given by the submanifolds

$$
R_{0}=\Delta_{M^{2}}, \quad R_{1}=\Delta_{M}^{(4)}
$$

Hence, using also (5-61),

$$
\begin{aligned}
& \operatorname{virdim} \mu_{E}\left(x_{1}, x_{2} ; y\right)=\mu^{\Delta_{M^{2}}}(x)-\mu^{\Delta_{M}^{(4)}}(z)-\left(\operatorname{dim} \Delta_{M^{2}}-\operatorname{dim} \Delta_{M^{2}} \cap \Delta_{M}^{(4)}\right) \\
& =\mu^{\Lambda}\left(x_{1}\right)+\mu^{\Lambda}\left(x_{2}\right)-\mu^{\Theta}(y)-n \text {. }
\end{aligned}
$$

This proves the part of Proposition 3.6 about $\mu_{E}$.

The space $M_{G} \quad$ Let $y \in \mathscr{P}^{\Theta}\left(H_{1} \oplus H_{2}\right)$ and $z \in \mathscr{P}^{\Lambda}\left(H_{1} \# H_{2}\right)$ (see Section 3.4). We set $Q=M \times M$, and

$$
\begin{aligned}
x:[0,1] \rightarrow T^{*} Q, & x(t):=\left(\mathscr{b} y_{1}(1-t), y_{2}(t)\right), \\
w:[0,1] \rightarrow T^{*} Q, & w(t):=(\mathscr{b} z((1-t) / 2), z((1+t) / 2)) .
\end{aligned}
$$

As in the case of $\mu_{E}$, the curve $x$ belongs to $\mathscr{P} \Delta_{M}^{(4)}(K)$, where the Hamiltonian $K$ is defined by (5-52), and

$$
\mu^{\Delta_{M}^{(4)}}(x)=\mu^{\Delta_{M}^{(4)}}(y)=\mu^{\Theta}(y) .
$$

On the other hand, by Lemma 5.32, $w$ belongs to $\mathscr{P}^{\Delta_{M} \times \Delta_{M}}(K)$ and

$$
\mu^{\Delta_{M} \times \Delta_{M}}(w)=\mu^{\Delta_{M}}(z)=\mu^{\Lambda}(z) .
$$

Then Corollary 5.26 implies that

$$
\begin{aligned}
& \operatorname{virdim} \mu_{G}(y, z)=\mu^{\Delta_{M}^{(4)}}(x)-\mu^{\Delta_{M} \times \Delta_{M}}(w)-\left(\operatorname{dim} \Delta_{M}^{(4)}-\operatorname{dim} \Delta_{M}^{(4)} \cap\left(\Delta_{M} \times \Delta_{M}\right)\right) \\
& =\mu^{\Theta}(y)-\mu^{\Lambda}(z) \text {. }
\end{aligned}
$$

This concludes the proof of Proposition 3.6. 
The space $\mu_{G E}^{\Upsilon}$ Let $x_{1} \in \mathscr{P}^{\Lambda}\left(H_{1}\right), x_{2} \in \mathscr{P} \Lambda\left(H_{2}\right)$, and $z \in \mathscr{P} \Lambda\left(H_{1} \# H_{2}\right)$ (see the proof of Theorem 3.8). The space $\mu_{G E}^{\Upsilon}\left(x_{1}, x_{2} ; z\right)$ is the set of pairs $(\alpha, u)$ where $\alpha$ is a real positive parameter and $u$ is a solution of the Floer equation on the Riemann surface $\Sigma_{G E}^{\Upsilon}(\alpha)$ with asymptotics $x_{1}, x_{2}, z$, and suitable nonlocal boundary conditions. Let $Q=M^{2}, K$ be as in (5-52), $x$ be as in (5-60), and

$$
y(t):=(\mathscr{C} z((1-t) / 2), z((1+t) / 2)),
$$

so that $x \in \mathscr{P}^{\Delta_{M}}(K)$ and $y \in \mathscr{P}^{\Delta_{M} \times \Delta_{M}}(K)$. For fixed $\alpha>0$, the set $\mu_{G E}^{\Upsilon}\left(x_{1}, x_{2} ; z\right)$ is in one-to-one correspondence with the space $M(x, y)$ of Corollary 5.26, where the boundary conditions jump at $s_{1}=0$ and $s_{2}=\alpha$, and are given by

$$
R_{0}=\Delta_{M^{2}}, \quad R_{1}=\Delta_{M}^{(4)}, \quad R_{2}=\Delta_{M} \times \Delta_{M} .
$$

Considering also the parameter $\alpha$, we deduce that $\mu_{G E}^{\Upsilon}\left(x_{1}, x_{2} ; z\right)$ has virtual dimension

$$
\begin{aligned}
& \operatorname{virdim} M_{G E}^{\Upsilon}\left(x_{1}, x_{2} ; z\right) \\
&=1+\operatorname{virdim} \mu(x, y) \\
&=1+\mu^{\Delta_{M}}(x)-\mu^{\Delta_{M} \times \Delta_{M}}(y) \\
&-\left(\operatorname{dim} \Delta_{M^{2}}-\operatorname{dim} \Delta_{M^{2}} \cap \Delta_{M}^{(4)}+\operatorname{dim} \Delta_{M}^{(4)}-\operatorname{dim} \Delta_{M}^{(4)} \cap\left(\Delta_{M} \times \Delta_{M}\right)\right) \\
&= 1+\mu^{\Lambda}\left(x_{1}\right)+\mu^{\Lambda}\left(x_{2}\right)-\mu^{\Lambda}(z)-n .
\end{aligned}
$$

In the last identity we have used also Lemma 5.31 and Lemma 5.32. This proves Proposition 3.9.

The spaces $\mu_{\mathrm{C}}$ and $\mu_{\mathrm{Ev}}$. Let $f$ be a Morse function on $M$, let $x$ be a critical point of $f$, and let $y \in \mathscr{P}^{\Lambda}(H)$. Given $q \in M$, let

$$
\begin{array}{r}
\widetilde{M}_{C}(q, y):=\left\{u \in C ^ { \infty } \left(\left[0,+\infty\left[\times \mathbb{T}, T^{*} M\right) \mid \bar{\partial}_{J, H}(u)=0, \pi \circ u(0, t) \equiv q \forall t \in \mathbb{T},\right.\right.\right. \\
\left.\lim _{s \rightarrow+\infty} u(s, t)=y(t)\right\} .
\end{array}
$$

For a fixed $q \in M$, the space $\widetilde{M}_{\mathrm{C}}(q, y)$ coincides with $M(q, y)$ from Corollary 5.29, where $k=0$ and $R_{0}=\Delta_{M}$. By Corollary 5.29, $\widetilde{M}_{\mathrm{C}}(q, y)$ has virtual dimension $-\mu^{\Delta_{M}}(y)=-\mu^{\Lambda}(y)$. Therefore, the space

has virtual dimension

$$
\mu_{\mathrm{C}}(x, y)=\bigcup_{q \in W^{u}(x)} \widetilde{M}_{C}(q, y),
$$

$$
\operatorname{virdim} \mu_{\mathrm{C}}(x, y)=\operatorname{dim} W^{u}(x)-\mu^{\Lambda}(y)=i(x)-\mu^{\Lambda}(y),
$$

proving the first part of Proposition 3.11. 
The space of maps

$$
\begin{array}{r}
\left.\widetilde{M}_{\mathrm{Ev}}(y):=\left\{u \in C^{\infty}(]-\infty, 0\right] \times \mathbb{T}, T^{*} M\right) \mid \bar{\partial}_{J, H}(u)=0, u(0, t) \in \mathbb{O}_{M} \forall t \in \mathbb{T}, \\
\left.\lim _{s \rightarrow-\infty} u(s, t)=y(t)\right\}
\end{array}
$$

can be identified with $M(y)$ from Corollary 5.30, where the boundary condition has no jumps and is given by $R_{0}=\Delta_{M}$. Hence

$$
\operatorname{virdim} \widetilde{M}_{\mathrm{Ev}}(y)=\mu^{\Delta_{M}}(y)=\mu^{\Lambda}(y) .
$$

Therefore, given $x$ a critical point of the Morse function $f$ on $M$, the space

$$
\mu_{\mathrm{Ev}}(y, x)=\left\{u \in \widetilde{\mathcal{M}}_{\mathrm{Ev}}(y) \mid u(0,0) \in W^{s}(x)\right\}
$$

has virtual dimension

$$
\operatorname{virdim} \mu_{\mathrm{Ev}}(y, x)=\mu^{\Lambda}(y)-\operatorname{codim} W^{s}(x)=\mu^{\Lambda}(y)-i(x) .
$$

This concludes the proof of Proposition 3.11.

The space $M_{I !}$ Let $x \in \mathscr{P}^{\Lambda}(H)$ and $y \in \mathscr{P}^{\Omega}(H)$ (see Section 3.5). The set $M_{I !}(x, y)$ is immediately seen to be in one-to-one correspondence with the space $M(x, y)$ of Corollary 5.26, where the boundary conditions jump at $s_{1}=0$ from $R_{0}=\Delta_{M}$ to $R_{1}=\left\{\left(q_{0}, q_{0}\right)\right\}$. Therefore,

$$
\begin{aligned}
\operatorname{virdim} \mathcal{M}_{I_{!}}(x, y) & =\mu^{\Delta_{M}}(x)-\mu^{\left(q_{0}, q_{0}\right)}(y)-\left(\operatorname{dim} \Delta_{M}-\operatorname{dim} \Delta_{M} \cap\left\{\left(q_{0}, q_{0}\right)\right\}\right) \\
& =\mu^{\Lambda}(x)-\mu^{\Omega}(y)-n .
\end{aligned}
$$

This proves Proposition 3.13.

The space $\mu_{\Upsilon}^{K}$ Let $\gamma_{1} \in \mathscr{P}^{\Omega}\left(L_{1}\right), \gamma_{2} \in \mathscr{P}^{\Omega}\left(L_{2}\right)$, and $x \in \mathscr{P}^{\Omega}\left(H_{1} \# H_{2}\right)$ (see Section 4.2). The space $\mu_{\Upsilon}^{K}\left(\gamma_{1}, \gamma_{2} ; x\right)$ consists of pairs $(\alpha, u)$ where $\alpha$ is a positive number and $u(s, t)$ is a solution of the Floer equation on the Riemann surface $\Sigma_{\Upsilon}^{K}(\alpha)$, which is asymptotic to $x$ for $s \rightarrow+\infty$, lies above some element in the unstable manifold of $\gamma_{1}$ (resp. $\gamma_{2}$ ) for $s=0$ and $-1 \leq t \leq 0^{-}$(resp. $0^{+} \leq t \leq 1$ ), and lies above $q_{0}$ at the other boundary points. Let us fix the two curves $q_{1}$ and $q_{2}$ in the unstable manifolds of $\gamma_{1}$ and $\gamma_{2}$ and the positive number $\alpha$. Set $Q=M^{2}$, let $K$ be the Hamiltonian on $[0,1] \times T^{*} Q$ defined by (5-52), and let $y:[0,1] \rightarrow T^{*} Q, \gamma:[0,1] \rightarrow Q$ be the curves

$$
y(t):=\left(\mathscr{b} x\left(\frac{1-t}{2}\right), x\left(\frac{1+t}{2}\right)\right), \quad \gamma(t):=\left(q_{1}(1-t), q_{2}(t)\right) .
$$


Lemma 5.32 implies that $y \in \mathscr{P}^{\Delta_{M} \times\left(q_{0}, q_{0}\right)}(K)$ and that

$$
\mu^{\Delta_{M} \times\left(q_{0}, q_{0}\right)}(y)=\mu^{\left(q_{0}, q_{0}\right)}(x)=\mu^{\Omega}(x) .
$$

Then the set of elements $(\alpha, u)$ in $\mathcal{M}_{\Upsilon}^{K}\left(\gamma_{1}, \gamma_{2} ; x\right)$ which lie above $q_{1}$ and $q_{2}$ for $s=0$ is in one-to-one correspondence with the space $M(\gamma, y)$ of Corollary 5.29, where the boundary conditions have a jump at $s_{1}=\alpha$ and are defined by

$$
R_{0}=\left\{\left(q_{0}, q_{0}, q_{0}, q_{0}\right)\right\}, \quad R_{1}=\Delta_{M} \times\left\{\left(q_{0}, q_{0}\right)\right\} .
$$

Such a space has virtual dimension

$$
\operatorname{virdim} M(\gamma, y)=-\mu^{\Delta_{M} \times\left(q_{0}, q_{0}\right)}(y)=-\mu^{\Omega}(x) .
$$

Letting the elements $q_{1}$ and $q_{2}$ of the unstable manifolds of $\gamma_{1}$ and $\gamma_{2}$ vary, we increase the virtual dimension by $i^{\Omega}\left(\gamma_{1} ; L_{1}\right)+i^{\Omega}\left(\gamma_{2} ; L_{2}\right)$. Letting also $\alpha$ vary we further increase the virtual dimension by 1 , and we find the formula

$$
\operatorname{virdim} \mu_{\Upsilon}^{K}\left(\gamma_{1}, \gamma_{2} ; x\right)=i^{\Omega}\left(\gamma_{1} ; L_{1}\right)+i^{\Omega}\left(\gamma_{2} ; L_{2}\right)-\mu^{\Omega}\left(x ; H_{1} \# H_{2}\right)+1 .
$$

This proves Proposition 4.2.

The space $\mu_{\alpha_{0}}^{K}$ Let $\gamma_{1} \in \mathscr{P} \Lambda\left(L_{1}\right), \gamma_{2} \in \mathscr{P} \Lambda\left(L_{2}\right)$, and $x \in \mathscr{P} \Theta\left(H_{1} \oplus H_{2}\right)$ (see Section 4.4). The space $\mu_{\alpha_{0}}^{K}\left(\gamma_{1}, \gamma_{2} ; x\right)$ consists of solutions $u=\left(u_{1}, u_{2}\right)$ of the Floer equation on the Riemann surface $\Sigma_{\alpha_{0}}^{K}$, which is asymptotic to $x$ for $s \rightarrow+\infty, u_{1}$ and $u_{2}$ lie above some elements $q_{1}$ and $q_{2}$ in the unstable manifolds of $\gamma_{1}$ and $\gamma_{2}$ for $s=0$, and $u$ satisfies the figure- 8 boundary condition for $s \geq \alpha_{0}$. Set $Q=M^{2}$, let $K$ be the Hamiltonian defined by (5-52), and let $y$ and $\gamma$ be as in (5-62). Then $y$ belongs to $\mathscr{P} \Delta_{M}^{(4)}(K)$, and

$$
\mu^{\Delta_{M}^{(4)}}(y)=\mu^{\Delta_{M}^{(4)}}(x)=\mu^{\Theta}(x) .
$$

The space of $u_{0} \in M_{\alpha_{0}}^{K}\left(\gamma_{1}, \gamma_{2} ; x\right)$ which lie above $q_{1}$ and $q_{2}$ for $s=0$ is in one-toone correspondence with the space $M(\gamma, y)$ of Corollary 5.29, where the boundary conditions jump at $s_{1}=\alpha_{0}$ and are given by

$$
R_{0}=\Delta_{M^{2}}, \quad R_{1}=\Delta_{M}^{(4)} .
$$

Such a space has virtual dimension

$$
\operatorname{virdim} \mu(\gamma, y)=-\mu^{\Delta_{M}^{(4)}}(y)-\left(\operatorname{dim} \Delta_{M^{2}}-\operatorname{dim} \Delta_{M^{2}} \cap \Delta_{M}^{(4)}\right)=-\mu^{\Theta}(x)-n .
$$

Letting the elements $q_{1}$ and $q_{2}$ of the unstable manifolds of $\gamma_{1}$ and $\gamma_{2}$ vary, we increase the virtual dimension by $i^{\Lambda}\left(\gamma_{1} ; L_{1}\right)+i^{\Lambda}\left(\gamma_{2} ; L_{2}\right)$, and we find the formula

$$
\operatorname{virdim} \mu_{\alpha_{0}}^{K}\left(\gamma_{1}, \gamma_{2} ; x\right)=i^{\Lambda}\left(\gamma_{1} ; L_{1}\right)+i^{\Lambda}\left(\gamma_{2} ; L_{2}\right)-\mu^{\Theta}(x)-n
$$

This proves Proposition 4.5. 
The space $M_{G}^{K} \quad$ Let $\gamma \in \mathscr{P}^{\Theta}\left(L_{1} \oplus L_{2}\right)$ and $x \in \mathscr{P}^{\Lambda}\left(H_{1} \# H_{2}\right)$ (see Section 4.5). The space $\mu_{G}^{K}(\gamma, x)$ consists of pairs $(\alpha, u)$ where $\alpha$ is a positive number and $u(s, t)$ is a solution of the Floer equation on the Riemann surface $\Sigma_{G}^{K}(\alpha)$, which is asymptotic to $x$ for $s \rightarrow+\infty$, lies above some element $q=\left(q_{1}, q_{2}\right)$ in the unstable manifold of $\gamma$ for $s=0$, and satisfies the figure-8 boundary condition for $s \in[0, \alpha]$. Set $Q=M^{2}$, let $K$ be as in (5-52), let $y$ be as in (5-62), and let $\tilde{\gamma}:[0,1] \rightarrow Q$ be the curve

$$
\tilde{\gamma}(t):=\left(q_{1}(1-t), q_{2}(t)\right) .
$$

Then the space of elements $(\alpha, u)$ in $\mu_{G}^{K}(\gamma, x)$ with $u$ above $q$ at $s=0$ is in one-toone correspondence with the space $M(\tilde{\gamma}, y)$ of Corollary 5.29, where the boundary conditions jump at $s_{1}=\alpha$ from $R_{0}=\Delta_{M}^{(4)}$ to $R_{1}=\Delta_{M} \times \Delta_{M}$. Such a space has virtual dimension

$$
\begin{aligned}
\operatorname{virdim} \mu(\tilde{\gamma}, y) & =-\mu^{\Delta_{M} \times \Delta_{M}}(y)-\left(\operatorname{dim} \Delta_{M}^{(4)}-\operatorname{dim} \Delta_{M}^{(4)} \cap\left(\Delta_{M} \times \Delta_{M}\right)\right) \\
& =-\mu^{\Lambda}(x) .
\end{aligned}
$$

where we have used Lemma 5.32. Letting the elements $q$ of the unstable manifold of $\gamma$ vary, we increase the virtual dimension by $i^{\Theta}\left(\gamma ; L_{1} \oplus L_{2}\right)$. Letting also $\alpha$ vary we further increase it by 1 , and we find the formula

$$
\operatorname{virdim} \mu_{G}^{K}(\gamma, x)=i^{\Theta}\left(\gamma ; L_{1} \oplus L_{2}\right)-\mu^{\Lambda}\left(x ; H_{1} \# H_{2}\right)+1 .
$$

This proves Proposition 4.8.

\section{Compactness and cobordism}

The first aim of this section is to explain how compactness and removal of singularities for Cauchy-Riemann problems can be obtained in the framework of cotangent bundles and conormal boundary conditions. The second aim is to prove the three already stated cobordism results (Propositions 3.10, 4.3 and 4.7) which do not follow form standard arguments in Floer theory.

\subsection{Compactness in the case of jumping conormal boundary conditions}

Compactness in the $C_{\mathrm{loc}}^{\infty}$ topology of all the spaces of solutions of the Floer equation considered in this paper can be proved within the following general setting. Let $Q$ be a closed Riemannian manifold, and let $R_{0}, R_{1}, \ldots, R_{k}$ be submanifolds of $Q \times Q$. We assume that there is an isometric embedding $Q \hookrightarrow \mathbb{R}^{N}$ and linear subspaces $V_{0}, V_{1}, \ldots, V_{k}$ of $\mathbb{R}^{N} \times \mathbb{R}^{N}$, such that $V_{j-1}$ is partially orthogonal to $V_{j}$, for every $j=1, \ldots, k$, and

$$
R_{j}=V_{j} \cap(Q \times Q) .
$$


The embedding $Q \hookrightarrow \mathbb{R}^{N}$ induces an embedding $T^{*} Q \hookrightarrow T^{*} \mathbb{R}^{N} \cong \mathbb{R}^{2 N} \cong \mathbb{C}^{N}$. Since the embedding $Q \hookrightarrow \mathbb{R}^{N}$ is isometric, the standard complex structure $J_{0}$ of $\mathbb{R}^{2 N}$ restricts to the metric almost complex structure $J$ on $T^{*} Q$. Let $H \in C^{\infty}\left([0,1] \times T^{*} Q\right)$ be a Hamiltonian satisfying (H1) and (H2). Fix real numbers

$$
-\infty=s_{0}<s_{1}<\cdots<s_{k}<s_{k+1}=+\infty,
$$

and let $u: \Sigma=\{z \in \mathbb{C} \mid 0 \leq \operatorname{Im} z \leq 1\} \rightarrow T^{*} Q$ be a solution of the Floer equation $\bar{\partial}_{J, H}(u)=0$ which satisfies the nonlocal boundary conditions

$$
(u(s), \mathscr{b} u(s+i)) \in N^{*} R_{j} \quad \forall s \in\left[s_{j-1}, s_{j}\right],
$$

for every $j=0, \ldots, k$.

The map $u$ satisfies the energy identity

$$
\begin{aligned}
\int_{a}^{b} \int_{0}^{1}\left|\partial_{s} u(s, t)\right|^{2} d t d s=\mathbb{A}_{H}(u(a, \cdot))-\mathbb{A}_{H}(u(b, \cdot)) \\
\quad+\int_{[a, b]}\left(u(\cdot, 1)^{*} \eta-u(\cdot, 0)^{*} \eta\right)=\mathbb{A}_{H}(u(a, \cdot))-\mathbb{A}_{H}(u(b, \cdot)),
\end{aligned}
$$

for every $a<b$, where the integral over $[a, b]$ vanishes because of the boundary conditions (6-1), thanks to the fact that $\eta \oplus \eta$, that is the Liouville form on $T^{*} Q^{2}$, vanishes on $N^{*} R_{j}$. The following result is proven in [4, Lemma 1.12] (in that lemma different boundary conditions are considered, but the proof makes use only of the energy identity (6-2) coming from those boundary conditions).

Lemma 6.1 For every $a>0$ there exists $c>0$ such that for every solution $u: \Sigma \rightarrow$ $T^{*} Q$ of $\bar{\partial}_{J, H}(u)=0$, with boundary conditions (6-1), and energy bound

$$
\int_{\Sigma}\left|\partial_{s} u(s, t)\right|^{2} d s d t \leq a,
$$

we have the estimates

$$
\|u\|_{L^{2}(I \times] 0,1[)} \leq c|I|^{1 / 2}, \quad\|\nabla u\|_{L^{2}(I \times] 0,1[)} \leq c\left(1+|I|^{1 / 2}\right),
$$

for every interval $I$.

The proof of the following result follows the argument of [4, Theorem 1.14], using the above lemma together with the elliptic estimates of Lemma 5.10. 
Proposition 6.2 For every $a>0$ there exists $c>0$ such that for every solution $u: \Sigma \rightarrow T^{*} Q$ of $\bar{\partial}_{J, H}(u)=0$, with boundary conditions (6-1), and energy bound

$$
\int_{\Sigma}\left|\partial_{s} u(s, t)\right|^{2} d s d t \leq a,
$$

we have the uniform estimate

$$
\|u\|_{L^{\infty}(\mathbb{R} \times] 0,1[D} \leq c .
$$

Proof By using the above embedding, the equation $\bar{\partial}_{J, H}(u)=0$ can be rewritten as

$$
\bar{\partial} u=J_{0} X_{H}(t, u) .
$$

We can pass to local boundary conditions by considering the map $v: \Sigma \rightarrow T^{*} Q^{2} \subset \mathbb{C}^{2 N}$ defined by

$$
v(z):=(\mathscr{b} u((i-z) / 2), u((i+z) / 2))=(\bar{u}((i-z) / 2), u((i+z) / 2)) .
$$

The map $v$ satisfies the boundary conditions

$$
\begin{aligned}
v(s) & \in N^{*} \Delta_{Q} \subset N^{*} \Delta_{\mathbb{R}^{N}}, & & \forall s \in \mathbb{R}, \\
v(s+i) & \in N^{*} R_{j} \subset N^{*} V_{j}, & & \text { if } s \in\left[2 s_{j-1}, 2 s_{j}\right] .
\end{aligned}
$$

Moreover,

$$
\bar{\partial} v(z)=\frac{1}{2}(\overline{\bar{\partial}} u((i-z) / 2), \bar{\partial} u((i+z) / 2)),
$$

so by (6-3) and by the fact that $X_{H}(t, q, p)$ has quadratic growth in $|p|$ by (3-2), there is a constant $c$ such that

$$
|\bar{\partial} v(z)| \leq c\left(1+|v(z)|^{2}\right) .
$$

Let $\chi$ be a smooth function such that $\chi(s)=1$ for $s \in[0,1], \chi(s)=0$ outside $[-1,2]$, and $0 \leq \chi \leq 1$. Given $h \in \mathbb{Z}$ set

$$
w(s+i t):=\chi(s-h) v(s+i t) .
$$

Fix some $p>2$, and consider the norm $\|\cdot\|_{X^{p}}$ introduced in Section 5.3, with $\mathscr{S}=\left\{2 s_{1}, \ldots, 2 s_{k}\right\}$. The map $w$ has compact support and satisfies the boundary conditions (6-4), so by Lemma 5.10 we have the elliptic estimate

$$
\|\nabla w\|_{X^{p}} \leq c_{0}\|w\|_{X^{p}}+c_{1}\|\bar{\partial} w\|_{X^{p}} .
$$

Since

$$
\bar{\partial} w=\chi^{\prime}(s-h) v+\chi(s-h) \bar{\partial} v=\frac{\chi^{\prime}}{\chi}(s-h) w+\chi(s-h) \bar{\partial} v,
$$


we obtain, together with (6-5),

$$
\begin{aligned}
\|\nabla w\|_{X^{p}} & \leq\left(c_{0}+c_{1}\left\|\chi^{\prime} / \chi\right\|_{\infty}\right)\|w\|_{X^{p}}+c_{1}\|\chi(\cdot-h) \bar{\partial} v\|_{X^{p}} \\
& \leq\left(c_{0}+c_{1}\left\|\chi^{\prime} / \chi\right\|_{\infty}\right)\|w\|_{X^{p}}+c_{1} c\left\|\chi(\cdot-h)\left(1+|v|^{2}\right)\right\|_{X^{p}} .
\end{aligned}
$$

Therefore, we have an estimate of the form

$$
\|\nabla w\|_{X^{p}} \leq a\|w\|_{X^{p}}+b\left\|\chi(\cdot-h)\left(1+|v|^{2}\right)\right\|_{X^{p}} .
$$

Since $w$ has support in the set $[h-1, h+2] \times[0,1]$, we can estimate its $X^{p}$ norm in terms of its $X^{1,2}$ norm, by Proposition 5.13. The $X^{1,2}$ norm is equivalent to the $W^{1,2}$ norm, and the latter norm is bounded by Lemma 6.1. We conclude that $\|w\|_{X^{p}}$ is uniformly bounded. Similarly, the $X^{p}$ norm of $\chi(\cdot-h)\left(1+|v|^{2}\right)$ is controlled by its $W^{1,2}$ norm, which is also bounded because of Lemma 6.1. Therefore, (6-6) implies that $w$ is uniformly bounded in $X^{1, p}$. Since $p>2$, we deduce that $w$ is uniformly bounded in $L^{\infty}$. The integer $h$ was arbitrary, hence we conclude that $v$ is uniformly bounded in $L^{\infty}$, and so is $u$.

We conclude this section by discussing how the above result leads to $C_{\text {loc }}^{\infty}$ compactness for the spaces of maps considered in this paper. We consider the model case of $\mu_{\Upsilon}^{\Lambda}\left(x_{1}, x_{2} ; y\right)$, the other cases being analogous.

By the equivalent description of $\mu_{\Upsilon}^{\Lambda}\left(x_{1}, x_{2} ; y\right)$ of Section 5.10 (see in particular (5-57)), the space of maps we are considering fits into the above setting. Indeed, an isometric embedding of $M$ into $\mathbb{R}^{N}$ induces isometric embeddings of $Q=M^{2}$ into $\mathbb{R}^{2 N}$ and of $Q^{2}$ into $\mathbb{R}^{4 N}$, such that $\Delta_{M^{2}}$ and $\Delta_{M} \times \Delta_{M}$ are mapped into $Q^{2} \cap \Delta_{\mathbb{R}^{2 N}}$ and $Q^{2} \cap\left(\Delta_{\mathbb{R}^{N}} \times \Delta_{\mathbb{R}^{N}}\right)$, where the linear subspaces $\Delta_{\mathbb{R}^{2 N}}$ and $\Delta_{\mathbb{R}^{N}} \times \Delta_{\mathbb{R}^{N}}$ are partially orthogonal. Therefore, the energy estimate (3-18) and Proposition 6.2 imply that the elements of $\mu_{\Upsilon}^{\Lambda}\left(x_{1}, x_{2} ; y\right)$ have a uniform $L^{\infty}$ bound.

For the remaining part of the argument leading to the $C_{\text {loc }}^{\infty}$ compactness of $\mu_{\Upsilon}^{\Lambda}\left(x_{1}, x_{2} ; y\right)$ it is more convenient to use the original definition of this solutions space and the smooth structure of $\Sigma_{\Upsilon}^{\Lambda}$. Then the argument is absolutely standard: If by contradiction there is no uniform $C^{1}$ bound, a concentration argument (see eg [32, Theorem 6.8]) produces a nonconstant $J$-holomorphic sphere. However, there are no nonconstant $J$-holomorphic spheres on cotangent bundles, because the symplectic form $\omega$ is exact. This contradiction proves the $C^{1}$ bound. Then the $C^{k}$ bounds for arbitrary $k$ follow from elliptic bootstrap, as in [32, Section 6.4].

Other solutions spaces, such as the space $\mu_{\Upsilon}^{\Omega}$ for the triangle products, involve Riemann surfaces with boundary, and the solutions take value on some conormal subbundle of $T^{*} M$. In this case the concentration argument for proving the $C^{1}$ bound could 
produce a nonconstant $J$-holomorphic disk with boundary on the given conormal subbundle. However, the Liouville one-form vanishes on conormal subbundles, so such $J$-holomorphic disks do not exist. Again we find a contradiction, leading to $C^{1}$ bounds and - by elliptic bootstrap - to $C^{k}$ bounds for every $k$.

\subsection{Removal of singularities}

Removal of singularities results state that isolated singularities of a $J$-holomorphic map with bounded energy can be removed (see for instance [38, Section 4.5]). In Proposition 6.4 below, we prove a result of this sort for corner singularities. The fact that we are dealing with cotangent bundles, which can be isometrically embedded into $\mathbb{C}^{N}$, allows to reduce such a statement to the following easy linear result, where $\mathbb{D}_{r}$ is the open disk of radius $r$ in $\mathbb{C}$, and $\mathbb{H}^{+}$is the upper right quadrant $\{\operatorname{Re} z>0, \operatorname{Im} z>0\}$.

Lemma 6.3 Let $V_{0}$ and $V_{1}$ be partially orthogonal linear subspaces of $\mathbb{R}^{n}$. Let $u: \operatorname{Cl}\left(\mathbb{D}_{1} \cap \mathbb{H}^{+}\right) \backslash\{0\} \rightarrow \mathbb{C}^{n}$ be a smooth map such that

$$
u \in L^{p}\left(\mathbb{D}_{1} \cap \mathbb{H}^{+}, \mathbb{C}^{n}\right), \quad \bar{\partial} u \in L^{p}\left(\mathbb{D}_{1} \cap \mathbb{H}^{+}, \mathbb{C}^{n}\right),
$$

for some $p>2$, and

$$
u(s) \in N^{*} V_{0} \quad \forall s>0, \quad u(i t) \in N^{*} V_{1} \quad \forall t>0 .
$$

Then $u$ extends to a continuous map on $\mathrm{Cl}\left(\mathbb{D}_{1} \cap \mathbb{H}^{+}\right)$.

Proof Since $V_{0}$ and $V_{1}$ are partially orthogonal, by applying twice the Schwarz reflection argument of the proof of Lemma 5.6 we can extend $u$ to a continuous map

$$
u: \mathbb{D}_{1} \backslash\{0\} \rightarrow \mathbb{C}^{n},
$$

which is smooth on $\mathbb{D}_{1} \backslash(\mathbb{R} \cup i \mathbb{R})$, has finite $L^{p}$ norm on $\mathbb{D}_{1}$, and satisfies

$$
\bar{\partial} u \in L^{p}\left(\mathbb{D}_{1}\right) .
$$

Since $p>2$, the $L^{2}$ norm of $u$ on $\mathbb{D}_{1}$ is also finite, and by the conformal change of variables $z=s+i t=e^{\zeta}=e^{\rho+i \theta}$, this norm can be written as

$$
\int_{\mathbb{D}_{1}}|u(z)|^{2} d s d t=\int_{-\infty}^{0} \int_{0}^{2 \pi}\left|u\left(e^{\rho+i \theta}\right)\right|^{2} e^{2 \rho} d \theta d \rho .
$$

The fact that this quantity is finite implies that there is a sequence $\rho_{h} \rightarrow-\infty$ such that, setting $\epsilon_{h}:=e^{\rho_{h}}$, we have

$$
\lim _{h \rightarrow \infty} \epsilon_{h}^{2} \int_{0}^{2 \pi}\left|u\left(\epsilon_{h} e^{i \theta}\right)\right|^{2} d \theta=\lim _{h \rightarrow \infty} e^{2 \rho_{h}} \int_{0}^{2 \pi}\left|u\left(e^{\rho_{h}+i \theta}\right)\right|^{2} d \theta=0 .
$$


If $\varphi \in C_{c}^{\infty}\left(\mathbb{D}_{1}, \mathbb{C}^{N}\right)$, an integration by parts using the Gauss formula leads to

$$
\begin{aligned}
\int_{\mathbb{D}_{1}}\langle u, \partial \varphi\rangle d s d t & =\int_{\mathbb{D}_{\epsilon_{h}}}\langle u, \partial \varphi\rangle d s d t+\int_{\mathbb{D}_{1} \backslash \mathbb{D}_{\epsilon_{h}}}\langle u, \partial \varphi\rangle d s d t \\
& =\int_{\mathbb{D}_{\epsilon_{h}}}\langle u, \partial \varphi\rangle d s d t-\int_{\mathbb{D}_{1} \backslash \mathbb{D}_{\epsilon_{h}}}\langle\bar{\partial} u, \varphi\rangle d s d t+i \int_{\partial \mathbb{D}_{\epsilon_{h}}}\langle u, \varphi\rangle d z
\end{aligned}
$$

Since $u$ and $\bar{\partial} u$ are integrable over $\mathbb{D}_{1}$, the first integral in the latter expression tends to zero, while the second one tends to

$$
-\int_{\mathbb{D}_{1}}\langle\bar{\partial} u, \varphi\rangle d s d t
$$

As for the last integral, we have

$$
\int_{\partial \mathbb{D}_{\epsilon_{h}}}\langle u, \varphi\rangle d z=i \epsilon_{h} \int_{0}^{2 \pi}\left\langle u\left(\epsilon_{h} e^{i \theta}\right), \varphi\left(\epsilon_{h} e^{i \theta}\right)\right\rangle e^{i \theta} d \theta,
$$

so by the Cauchy-Schwarz inequality,

$$
\begin{aligned}
\left|\int_{\partial \mathbb{D}_{\epsilon_{h}}}\langle u, \varphi\rangle d z\right| & \leq \epsilon_{h}\left(\int_{0}^{2 \pi}\left|u\left(\epsilon_{h} e^{i \theta}\right)\right|^{2} d \theta\right)^{1 / 2}\left(\int_{0}^{2 \pi}\left|\varphi\left(\epsilon_{h} e^{i \theta}\right)\right|^{2} d \theta\right)^{1 / 2} \\
& \leq \sqrt{2 \pi} \epsilon_{h}\left(\int_{0}^{2 \pi}\left|u\left(\epsilon_{h} e^{i \theta}\right)\right|^{2} d \theta\right)^{1 / 2}\|\varphi\|_{\infty} .
\end{aligned}
$$

Then (6-7) implies that the latter quantity tends to zero for $h \rightarrow \infty$. Therefore,

$$
\int_{\mathbb{D}_{1}}\langle u, \partial \varphi\rangle d s d t=-\int_{\mathbb{D}_{1}}\langle\bar{\partial} u, \varphi\rangle d s d t
$$

for every test function $\varphi \in C_{c}^{\infty}\left(\mathbb{D}_{1}, \mathbb{C}^{n}\right)$. Since $\bar{\partial} u \in L^{p}$, by the regularity theory of the weak solutions of $\frac{\bar{\partial}}{\partial}$ (see Theorem 5.4 (i)), $u$ belongs to $W^{1, p}\left(\mathbb{D}_{1}, \mathbb{C}^{n}\right)$. Since $p>2$, we conclude that $u$ is continuous at 0 .

Let $R_{0}$ and $R_{1}$ be closed submanifolds of $Q$, and assume that there is an isometric embedding $Q \hookrightarrow \mathbb{R}^{N}$ such that

$$
R_{0}=Q \cap V_{0}, \quad R_{1} \cap V_{1},
$$

where $V_{0}$ and $V_{1}$ are partially orthogonal linear subspaces of $\mathbb{R}^{N}$. 
Proposition 6.4 Suppose $X: \mathbb{D}_{1} \cap \mathbb{H}^{+} \times T^{*} Q \rightarrow T T^{*} Q$ is a smooth vector field such that $X(z, q, p)$ grows at most polynomially in $p$, uniformly in $(z, q)$. Let $u: \operatorname{Cl}\left(\mathbb{D}_{1} \cap \mathbb{H}^{+}\right) \backslash\{0\} \rightarrow T^{*} Q$ be a smooth solution of the equation

$$
\bar{\partial}_{J}(u)(z)=X(z, u(z)) \quad \forall z \in \operatorname{Cl}\left(\mathbb{D}_{1} \cap \mathbb{H}^{+}\right) \backslash\{0\},
$$

such that

$$
u(s) \in N^{*} R_{0} \quad \forall s>0, \quad u(i t) \in N^{*} R_{1} \quad \forall t>0 .
$$

If $u$ has finite energy,

$$
\int_{\mathbb{D}_{1} \cap \mathbb{H}^{+}}|\nabla u|^{2} d s d t<+\infty
$$

then $u$ extends to a continuous map on $\mathrm{Cl}\left(\mathbb{D}_{1} \cap \mathbb{H}^{+}\right)$.

Proof By means of the above isometric embedding, we may regard $u$ as a $\mathbb{C}^{N}$-valued map, satisfying the equation (6-8) with $\bar{\partial}_{J}=\bar{\partial}$, the energy estimate (6-9), and the boundary condition

$$
u(s) \in N^{*} V_{0} \quad \forall s>0, \quad u(i t) \in N^{*} V_{1} \quad \forall t>0 .
$$

By the energy estimate (6-9), $u$ belongs to $L^{p}\left(\mathbb{D}_{1} \cap \mathbb{H}^{+}, \mathbb{C}^{N}\right)$ for every $p<+\infty$ : for instance, this follows from the Poincaré inequality and the Sobolev embedding theorem on $\mathbb{D}_{1}$, after applying a Schwarz reflection twice and after multiplying by a cut-off function vanishing on $\partial \mathbb{D}_{1}$ and equal to 1 on a neighborhood of 0 . The polynomial growth of $X$ then implies that

$$
X(\cdot, u(\cdot)) \in L^{p}\left(\mathbb{D}_{1} \cap \mathbb{H}^{+}, \mathbb{C}^{N}\right) \quad \forall p<+\infty .
$$

Therefore, Lemma 6.3 implies that $u$ extends to a continuous map on $\operatorname{Cl}\left(\mathbb{D}_{1} \cap \mathbb{H}^{+}\right)$.

The corresponding statement for jumping conormal boundary conditions is:

Proposition 6.5 Suppose $X: \mathbb{D}_{1} \cap \mathbb{H} \times T^{*} Q \rightarrow T T^{*} Q$ is a smooth vector field such that $X(z, q, p)$ grows at most polynomially in $p$, uniformly in $(z, q)$. Let $u: \mathrm{Cl}\left(\mathbb{D}_{1} \cap \mathbb{H}\right) \backslash\{0\} \rightarrow T^{*} Q$ be a smooth solution of the equation

$$
\bar{\partial}_{J}(u)(z)=X(z, u(z)) \quad \forall z \in \mathrm{Cl}\left(\mathbb{D}_{1} \cap \mathbb{H}\right) \backslash\{0\},
$$

such that

$$
u(s) \in N^{*} R_{0} \quad \forall s>0, \quad u(s) \in N^{*} R_{1} \quad \forall s<0 .
$$

If $u$ has finite energy,

$$
\int_{\mathbb{D}_{1} \cap \mathbb{H}}|\nabla u|^{2} d s d t<+\infty,
$$

then $u$ extends to a continuous map on the closed half-disk $\mathrm{Cl}\left(\mathbb{D}_{1} \cap \mathbb{H}\right)$. 
Proof The energy is invariant with respect to conformal changes of variable. Therefore, it is enough to apply Proposition 6.4 to the map $v(z)=u\left(z^{2}\right)$, with $z \in \mathbb{D}_{1} \cap \mathbb{H}^{+}$.

\subsection{Proof of Proposition 3.10}

Let $x_{1} \in \mathscr{P}^{\Lambda}\left(H_{1}\right), x_{2} \in \mathscr{P}^{\Lambda}\left(H_{2}\right)$, and $z \in \mathscr{P}^{\Lambda}\left(H_{1} \# H_{2}\right)$ be such that

$$
\mu^{\Lambda}\left(x_{1}\right)+\mu^{\Lambda}\left(x_{2}\right)-\mu^{\Lambda}(z)=n,
$$

so that the manifold $\mu_{G E}^{\Upsilon}\left(x_{1}, x_{2} ; z\right)$ is one-dimensional. By standard arguments, Proposition 3.10 is implied by the following two statements:

(i) For every $y \in \mathscr{P}^{\Theta}\left(H_{1} \oplus H_{2}\right)$ such that

$$
\mu^{\Theta}(y)=\mu^{\Lambda}(z)=\mu^{\Lambda}\left(x_{1}\right)+\mu^{\Lambda}\left(x_{2}\right)-n,
$$

and every pair $\left(u_{1}, u_{2}\right)$ with $u_{1} \in M_{E}\left(x_{1}, x_{2} ; y\right)$ and $u_{2} \in M_{G}(y, z)$, there exists a unique connected component of $\mu_{G E}^{\Upsilon}\left(x_{1}, x_{2} ; z\right)$ containing a curve $\alpha \mapsto\left(\alpha, u_{\alpha}\right)$ which - modulo translations in the $s$ variable - converges to $\left(+\infty, u_{1}\right)$ and to $\left(+\infty, u_{2}\right)$.

(ii) For every $u \in \mathcal{M}_{\Upsilon}^{\Lambda}\left(x_{1}, x_{2} ; z\right)$, there exists a unique connected component of $\mu_{G E}^{\Upsilon}\left(x_{1}, x_{2} ; z\right)$ containing a curve $\alpha \mapsto\left(\alpha, u_{\alpha}\right)$ which converges to $(0, u)$.

The first statement follows from standard gluing arguments. Here we prove the second statement, by reducing it to an implicit function type argument. At first the difficulty consists in the parameter dependence of the underlying domain for the elliptic PDE. By using the special form of the occurring conormal type boundary conditions and a suitable localization argument, we equivalently translate this parameter dependence into a continuous family of elliptic operators with fixed boundary conditions.

If $(\alpha, v) \in M_{G E}^{\Upsilon}\left(x_{1}, x_{2} ; z\right)$, we define the map

$$
u: \Sigma=\{z \in \mathbb{C} \mid 0 \leq \operatorname{Im} z \leq 1\} \rightarrow T^{*} M^{2}, \quad u(z):=(\mathscr{C} v(\bar{z}), v(z)),
$$

and the Hamiltonian $K$ on $\mathbb{T} \times T^{*} M^{2}$ by

$$
K\left(t, x_{1}, x_{2}\right):=H_{1}\left(-t, \mathscr{b} x_{1}\right)+H_{2}\left(t, x_{2}\right) .
$$

By this identification, we can view the space $\mu_{G E}^{\Upsilon}\left(x_{1}, x_{2} ; z\right)$ as the space of pairs $(\alpha, u)$, where $\alpha>0$ and $u: \Sigma \rightarrow T^{*} M^{2}$ solves the Floer equation

$$
\bar{\partial}_{J, K}(u)=0,
$$


with nonlocal boundary conditions

$$
(u(s), \mathscr{b} u(s+i)) \in \begin{cases}N^{*} \Delta_{M^{2}} & \text { if } s \leq 0, \\ N^{*} \Delta_{M}^{(4)} & \text { if } 0 \leq s \leq \alpha, \\ N^{*}\left(\Delta_{M} \times \Delta_{M}\right) & \text { if } s \geq \alpha,\end{cases}
$$

and asymptotics

$$
\begin{aligned}
& \lim _{s \rightarrow-\infty} u(s+i t)=\left(\mathscr{b} x_{1}(-t), x_{2}(t)\right), \\
& \lim _{s \rightarrow+\infty} u(s+i t)=(\mathscr{b} z((1-t) / 2), z((1+t) / 2)) .
\end{aligned}
$$

Similarly, we can view the space $\mu_{\Upsilon}^{\Lambda}\left(x_{1}, x_{2} ; z\right)$ as the space of maps $u: \Sigma \rightarrow T^{*} M^{2}$ solving the equation (6-12) with asymptotics (6-14) and nonlocal boundary conditions

$$
(u(s), \mathscr{C} u(s+i)) \in \begin{cases}N^{*} \Delta_{M^{2}} & \text { if } s \leq 0, \\ N^{*}\left(\Delta_{M} \times \Delta_{M}\right) & \text { if } s \geq 0 .\end{cases}
$$

Compactness We start with the following compactness results, which also clarifies the sense of the convergence in (ii):

Lemma 6.6 Let $\left(\alpha_{h}, u_{h}\right)$ be a sequence in $\mu_{G E}^{\Upsilon}\left(x_{1}, x_{2} ; z\right)$ with $\alpha_{h} \rightarrow 0$. Then there exists $u_{0} \in M_{\Upsilon}^{\Lambda}\left(x_{1}, x_{2} ; z\right)$ such that, up to a subsequence, $u_{h}$ converges to $u_{0}$ in $C_{\text {loc }}^{\infty}(\Sigma \backslash\{0, i\})$, in $C^{\infty}(\Sigma \cap\{|\operatorname{Re} z|>1\})$, and uniformly on $\Sigma$.

Proof Since the sequence of maps $\left(u_{h}\right)$ has uniformly bounded energy, Proposition 6.2 implies a uniform $L^{\infty}$ bound. Then, the usual non-bubbling-off analysis for interior points and boundary points away from the jumps in the boundary condition implies that, modulo subsequence, we have

$$
u_{h} \rightarrow u_{0} \quad \text { in } C_{\mathrm{loc}}^{\infty}\left(\Sigma \backslash\{0, i\}, T^{*} M^{2}\right),
$$

where $u_{0}$ is a smooth solution of equation (6-12) on $\Sigma \backslash\{0, i\}$ with bounded energy and satisfying the boundary conditions (6-15), except possibly at 0 and $i$. By Proposition 6.5, the singularities 0 and $i$ are removable, and $u_{0}$ satisfies the boundary condition also at 0 and $i$. By the index formula (6-11) and transversality, the sequence $u_{h}$ cannot split, so $u_{0}$ satisfies also the asymptotic conditions (6-14), and $u_{h} \rightarrow u_{0}$ in $C^{\infty}(\Sigma \cap\{|\operatorname{Re} z|>1\})$. Therefore, $u_{0}$ belongs to $\mu_{\Upsilon}^{\Lambda}\left(x_{1}, x_{2} ; z\right)$, and there remains to prove that $u_{h} \rightarrow u$ uniformly on $\Sigma$.

We assume by contraposition that $\left(u_{h}\right)$ does not converge uniformly on $\Sigma$. By Ascoli-Arzelà theorem, there must be some blow-up of the gradient. That is, modulo 
subsequence, we can find $z_{h} \in \Sigma$ converging either to 0 or to $i$ such that

$$
R_{h}:=\left|\nabla u_{h}\left(z_{h}\right)\right|=\left\|\nabla u_{h}\right\|_{\infty} \rightarrow \infty .
$$

For sake of simplicity, we only consider the case $z_{h}=\left(s_{h}, 0\right) \rightarrow 0,0<s_{h}<\alpha_{h} \rightarrow 0$. The general case follows along analogous arguments using additional standard bubbling-off arguments. For more details, see eg [32, Section 6.4].

We now have to make a case distinction concerning the behavior of the quantity $0<R_{h} \alpha_{h}<\infty$ :

(a) The case of a diverging subsequence $R_{h_{j}} \alpha_{h_{j}} \rightarrow \infty$ can be handled by conformal rescaling $v_{j}(s, t):=u_{h_{j}}\left(s_{h_{j}}+s / R_{h_{j}}, t / R_{h_{j}}\right)$ which provides us with a finite energy disk with boundary on a single Lagrangian submanifold of conormal type. This has to be constant due to the vanishing of the Liouville 1-form on conormals, contradicting the convergence of $\left|\nabla v_{j}(0)\right|=1$.

(b) The case of convergence of a subsequence $R_{h_{j}} \alpha_{h_{j}} \rightarrow 0$ can be dealt with by rescaling $v_{j}(s, t):=u_{h_{j}}\left(s_{h_{j}}+\alpha_{h_{j}}, \alpha_{h_{j}} t\right)$. Now $v_{k}$ has to converge uniformly on compact subsets towards a constant map, since $\left\|\nabla v_{j}\right\|_{\infty}=\left|\nabla v_{j}(0)\right|=R_{h_{j}} \alpha_{h_{j}} \rightarrow 0$. This in particular implies that $u_{h_{j}}(\cdot, 0)_{\mid\left[0, \alpha_{h_{j}}\right]}$ converges uniformly to a point contradicting the contraposition assumption.

(c) It remains to study the case $R_{h} \alpha_{h} \rightarrow c>0$. Again we rescale $v_{h}(s, t)=$ $u_{h}\left(\alpha_{h} s, \alpha_{h} t\right)$, which now has to converge to a nonconstant $J$-holomorphic map $v$ on the upper half plane. After applying a suitable conformal coordinate change and transforming the nonlocal boundary conditions into local ones, we can view $v$ as a map on the half strip $v: \Sigma^{+} \rightarrow T^{*} M^{4}$, satisfying the boundary conditions

$$
\begin{aligned}
v(i t) & \in N^{*} \Delta_{M}^{(4)} & & \text { for } t \in[0,1], \\
v(s) & \in N^{*}\left(\Delta_{M} \times \Delta_{M}\right) & & \text { for } s \geq 0, \\
v(s+i) & \in N^{*} \Delta_{M^{2}} & & \text { for } s \geq 0 .
\end{aligned}
$$

Applying again the removal of singularities for $s \rightarrow \infty$, we obtain $v$ as a $J$-holomorphic triangle with boundary on three conormals. Hence, $v$ would have to be constant, contradicting again the rescaling procedure.

This shows the uniform convergence of a subsequence of $\left(u_{h}\right)$.

Localization It is convenient to transform the nonlocal boundary conditions (6-13) and (6-15) into local boundary conditions, by the usual method of doubling the space: Given $u: \Sigma \rightarrow T^{*} M^{2}$ we define $\tilde{u}: \Sigma \rightarrow T^{*} M^{4}$ as

$$
\tilde{u}(z):=(u(z / 2), \mathscr{C} u(i+\bar{z} / 2)) .
$$


Then $u$ solves $\bar{\partial}_{J, K}(u)=0$ if and only if $\tilde{u}$ solves the equation

$$
\bar{\partial}_{J, \widetilde{K}}(\widetilde{u}),
$$

with upper boundary condition

$$
\tilde{u}(s+i) \in N^{*} \Delta_{M^{2}} \quad \forall s \in \mathbb{R},
$$

where the Hamiltonian $\tilde{K}:[0,1] \times T^{*} M^{4} \rightarrow \mathbb{R}$ is defined by

$$
\widetilde{K}\left(t, x_{1}, x_{2}, x_{3}, x_{4}\right):=\frac{1}{2} K\left(\frac{t}{2}, x_{1}, x_{2}\right)+\frac{1}{2} K\left(1-\frac{t}{2}, \mathscr{b} x_{3}, \mathscr{b} x_{4}\right) .
$$

Moreover, $u$ satisfies (6-13) if and only if $\tilde{u}$ satisfies

$$
\tilde{u}(s) \in \begin{cases}N^{*} \Delta_{M^{2}} & \text { if } s \leq 0, \\ N^{*} \Delta_{M}^{\Theta} & \text { if } 0 \leq s \leq 2 \alpha, \\ N^{*}\left(\Delta_{M} \times \Delta_{M}\right) & \text { if } s \geq 2 \alpha\end{cases}
$$

whereas $u$ satisfies (6-15) if and only if $\tilde{u}$ satisfies

$$
\tilde{u}(s) \in \begin{cases}N^{*} \Delta_{M^{2}} & \text { if } s \leq 0, \\ N^{*}\left(\Delta_{M} \times \Delta_{M}\right) & \text { if } s \geq 0 .\end{cases}
$$

Finally, the asymptotic condition (6-14) is translated into

$$
\begin{aligned}
& \lim _{s \rightarrow-\infty} \tilde{u}(s+t i)=\left(\mathscr{b} x_{1}(-t / 2), x_{2}(t / 2), x_{1}(t / 2-1), \mathscr{b} x_{2}(1-t / 2)\right), \\
& \lim _{s \rightarrow+\infty} \tilde{u}(s+t i)=(\mathscr{b} z(1 / 2-t / 4), z(1 / 2+t / 4), z(t / 4), \mathscr{b} z(1-t / 4)) .
\end{aligned}
$$

Let $u_{0} \in M_{\Upsilon}^{\Lambda}\left(x_{1}, x_{2} ; z\right)$. We must prove that there exists a unique connected component of $\mathcal{M}_{G E}^{\Upsilon}\left(x_{1}, x_{2} ; z\right)$ containing a curve $\alpha \mapsto\left(\alpha, u_{\alpha}\right)$ which converges to $\left(0, u_{0}\right)$, in the sense of Lemma 6.6.

Let $\tilde{u}_{0}$ be the map from $\Sigma$ to $T^{*} M^{4}$ associated to $u_{0}: \tilde{u}_{0}$ solves (6-16) with boundary conditions (6-17), (6-19), and asymptotic conditions (6-20). Since we are looking for solutions which converge to $\tilde{u}_{0}$ uniformly on $\Sigma$, we may localize the problem and assume that $M=\mathbb{R}^{n}$. More precisely, if the projection of $\tilde{u}_{0}(z)$ onto $M^{4}$ is $\left(q_{1}, q_{2}, q_{3}, q_{4}\right)(z)$, we construct open embeddings

$$
\Sigma \times \mathbb{R}^{n} \rightarrow \Sigma \times M, \quad(z, q) \mapsto\left(z, \varphi_{j}(z, q)\right), \quad j=1, \ldots, 4,
$$

such that $\varphi_{j}(z, 0)=q_{j}(z)$ and $D_{2} \varphi_{j}(z, 0)$ is an isometry, for every $z \in \Sigma$ (for instance, by composing an isometric trivialization of $q_{j}^{*}(T M)$ by the exponential mapping). 
The induced open embeddings

$$
\begin{aligned}
\Sigma \times T^{*} \mathbb{R}^{n} & \rightarrow \Sigma \times T^{*} M, \\
(z, q, p) & \mapsto\left(z, \psi_{j}(z, q, p)\right):=\left(z, \varphi_{j}(z, q),\left(D_{2} \varphi_{j}(z, q)^{*}\right)^{-1} p\right), \quad j=1, \ldots, 4,
\end{aligned}
$$

are the components of the open embedding

$$
\Sigma \times T^{*} \mathbb{R}^{4 n} \rightarrow \Sigma \times T^{*} M^{4}, \quad(z, \xi) \mapsto(z, \psi(z, \xi)):=\left(z, \psi_{1}\left(z, \xi_{1}\right), \ldots, \psi_{4}\left(z, \xi_{4}\right)\right) .
$$

Such an embedding allows us to associate to any $\tilde{u}: \Sigma \rightarrow T^{*} M^{4}$ which is $C^{0}$-close to $\tilde{u}_{0}$ a map $w: \Sigma \rightarrow T^{*} \mathbb{R}^{4 n}=\mathbb{C}^{4 n}$, by setting

$$
\tilde{u}(z)=\psi(z, w(z)) .
$$

Then $\tilde{u}$ solves (6-16) if and only if $w$ solves an equation of the form

$$
\mathscr{D}(w):=\partial_{s} w(z)+J(z, w(z)) \partial_{t} w(z)+G(z, w(z))=0,
$$

where $J$ is an almost complex structure on $\mathbb{C}^{4 n}$ parametrized on $\Sigma$ and such that $J(z, 0)=J_{0}$ for any $z \in \Sigma$, whereas $G: \Sigma \times \mathbb{C}^{4 n} \rightarrow \mathbb{C}^{4 n}$ is such that $G(z, 0)=0$ for any $z \in \Sigma$. Moreover, $\tilde{u}$ solves the asymptotic conditions (6-20) if and only if $w(s, t)$ tends to 0 for $s \rightarrow \pm \infty$. The maps $\psi_{j}(z, \cdot)$ preserve the Liouville form, so they map conormals into conormals. It easily follows that the boundary condition (6-17) on $\tilde{u}$ is translated into

$$
w(s+i) \in N^{*} \Delta_{\mathbb{R}^{2 n}} \quad \forall s \in \mathbb{R} .
$$

Moreover, $\tilde{u}$ satisfies the boundary condition (6-18) if and only if $w$ satisfies

$$
w(s) \in \begin{cases}N^{*} \Delta_{\mathbb{R}^{2 n}} & \text { if } s \leq 0, \\ N^{*} \Delta_{\mathbb{R}^{n}}^{(4)} & \text { if } 0 \leq s \leq 2 \alpha, \\ N^{*}\left(\Delta_{\mathbb{R}^{n}} \times \Delta_{\mathbb{R}^{n}}\right) & \text { if } s \geq 2 \alpha .\end{cases}
$$

Similarly, $\tilde{u}$ satisfies the boundary condition (6-19) if and only if $w$ satisfies

$$
w(s) \in \begin{cases}N^{*} \Delta_{\mathbb{R}^{2 n}} & \text { if } s \leq 0, \\ N^{*}\left(\Delta_{\mathbb{R}^{n}} \times \Delta_{\mathbb{R}^{n}}\right) & \text { if } s \geq 0 .\end{cases}
$$

The element $u_{0} \in \mathcal{M}_{\Upsilon}^{\Lambda}\left(x_{1}, x_{2} ; z\right)$ corresponds to the solution $w_{0}=0$ of (6-21)-(6-24). By using the functional setting introduced in Section 5, we can view the nonlinear operator $\mathscr{D}$ defined in (6-21) as a continuously differentiable operator

$$
\left.\mathscr{D}: X_{\mathscr{Y}, \mathscr{q}, \mathscr{V}^{\prime}}^{1,(\Sigma,}, \mathbb{C}^{4 n}\right) \rightarrow X_{\mathscr{Y}}^{p}\left(\Sigma, \mathbb{C}^{4 n}\right)
$$


where $\mathscr{Y}:=\{0\}, \mathscr{V}:=\left(\Delta_{\mathbb{R}^{2 n}}, \Delta_{\mathbb{R}^{n}} \times \Delta_{\mathbb{R}^{n}}\right), \mathscr{V}^{\prime}:=\left(\Delta_{\mathbb{R}^{2 n}}\right)$, and $p$ is some number larger than 2. Since $J(z, 0)=J_{0}$, the differential of $\mathscr{D}$ at $w_{0}=0$ is a linear operator of the kind studied in Section 5, and by the transversality assumption it is an isomorphism.

Consider the orthogonal decomposition

$$
\mathbb{R}^{4 n}=W_{1} \oplus W_{2} \oplus W_{3} \oplus W_{4},
$$

where

$$
W_{1}:=\Delta_{\mathbb{R}^{n}}^{(4)}=\Delta_{\mathbb{R}^{2 n}} \cap\left(\Delta_{\mathbb{R}^{n}} \times \Delta_{\mathbb{R}^{n}}\right), \quad \Delta_{\mathbb{R}^{2 n}}=W_{1} \oplus W_{2}, \quad \Delta_{\mathbb{R}^{n}} \times \Delta_{\mathbb{R}^{n}}=W_{1} \oplus W_{3},
$$

and denote by $P_{j}$ the orthogonal projection of $\mathbb{C}^{4 n}$ onto $N^{*} W_{j}=W_{j} \oplus i W_{j}^{\perp}$. If $\mathscr{T}_{\alpha}$ is the translation operator mapping some $w: \Sigma \rightarrow \mathbb{C}^{4 n}$ into

$$
\left(\mathscr{T}_{\alpha} w\right)(z):=\left(P_{1} w(z), P_{2} w(z), P_{3} w(z-2 \alpha), P_{4} w(z)\right),
$$

we easily see that $w$ satisfies the boundary conditions (6-24) if and only if $\mathscr{T}_{\alpha} w$ satisfies the boundary conditions (6-23). Therefore, if we define the operator

$$
\mathscr{D}_{\alpha}: X_{\mathscr{S}, \mathscr{V}, \mathscr{V}^{\prime}}^{1, p}\left(\Sigma, \mathbb{C}^{4 n}\right) \rightarrow X_{\mathscr{Y}}^{p}\left(\Sigma, \mathbb{C}^{4 n}\right), \quad \mathscr{D}_{\alpha}=\mathscr{D}_{0} \circ \mathscr{T}_{\alpha},
$$

we have that $w \in X_{\mathscr{Y}, \mathscr{Q}, V^{\prime}}^{1, p}\left(\Sigma, \mathbb{C}^{4 n}\right)$ solves $\mathscr{D}_{\alpha}(w)=0$ if and only if $\mathscr{T}_{\alpha} w$ is a solution of (6-21) satisfying the boundary conditions (6-22) and (6-23). The operator

$$
\left[0,+\infty\left[\times X_{\mathscr{S}, \mathscr{V}, \mathscr{V}^{\prime}}^{1, p}\left(\Sigma, \mathbb{C}^{4 n}\right) \rightarrow X_{\mathscr{Y}}^{p}\left(\Sigma, \mathbb{C}^{4 n}\right), \quad(\alpha, w) \mapsto \mathscr{D}_{\alpha}(w),\right.\right.
$$

is continuous on the product, it is continuously differentiable with respect to the second variable, and this partial differential is continuous on the product. Moreover, $D \mathscr{D}_{0}(0)=D \mathscr{D}(0)$ is an isomorphism, so the parametric inverse mapping theorem implies that there are a number $\alpha_{0}>0$ and a neighborhood $U$ of 0 in $X_{\mathscr{\varphi}, \mathcal{,}, \mathcal{V}^{\prime}}^{1, p}\left(\Sigma, \mathbb{C}^{4 n}\right)$, such that the set of zeroes in $\left[0, \alpha_{0}[\times \mathcal{U}\right.$ of the above operator consists of a continuous curve $\left[0, \alpha_{0}\left[\ni \alpha \rightarrow\left(\alpha, w_{\alpha}\right)\right.\right.$ starting at $w_{0}=0$. Then $\alpha \rightarrow\left(\alpha, \mathscr{T}_{\alpha} w_{\alpha}\right)$ provides us with the unique curve in $\mu_{G E}^{\Upsilon}\left(x_{1}, x_{2} ; z\right)$ converging to $\left(0, u_{0}\right)$. This concludes the proof of Proposition 3.10.

\subsection{Proof of Proposition 4.3}

Fix some $\gamma_{1} \in \mathscr{P}^{\Omega}\left(L_{1}\right), \gamma_{2} \in \mathscr{P}^{\Omega}\left(L_{2}\right)$, and $x \in \mathscr{P}^{\Omega}\left(H_{1} \# H_{2}\right)$ such that

$$
i^{\Omega}\left(\gamma_{1} ; L_{1}\right)+i^{\Omega}\left(\gamma_{2} ; L_{2}\right)-\mu^{\Omega}\left(x ; H_{1} \# H_{2}\right)=0 .
$$

By a standard argument in Floer homology, the claim that $P_{\Upsilon}^{K}$ is a chain homotopy between $K^{\Omega}$ and $\Upsilon^{\Omega} \circ\left(\Phi_{L_{1}}^{\Omega} \otimes \Phi_{L_{2}}^{\Omega}\right)$ is implied by the following statements: 
(i) For every $\left(u_{1}, u_{2}\right) \in \mu_{\Phi}^{\Omega}\left(\gamma_{1}, y_{1}\right) \times \mu_{\Phi}^{\Omega}\left(\gamma_{2}, y_{2}\right)$ and every $u \in \mu_{\Upsilon}^{\Omega}\left(y_{1}, y_{2} ; x\right)$, where $\left(y_{1}, y_{2}\right) \in \mathscr{P}^{\Omega}\left(H_{1}\right) \times \mathscr{P}^{\Omega}\left(H_{2}\right)$ is such that

$$
\mu^{\Omega}\left(y_{1} ; H_{1}\right)+\mu^{\Omega}\left(y_{2} ; H_{2}\right)=\mu^{\Omega}\left(x ; H_{1} \# H_{2}\right),
$$

there exists a unique connected component of $\mu_{\Upsilon}^{K}\left(\gamma_{1}, \gamma_{2} ; x\right)$ containing a curve $\left(\alpha, u_{\alpha}\right)$ such that $\alpha \rightarrow+\infty, u_{\alpha}(\cdot, \cdot-1)$ and $u_{\alpha}$ converge to $u_{1}$ and $u_{2}$, respectively, in $C_{\mathrm{loc}}^{\infty}\left(\left[0,+\infty\left[\times[0,1], T^{*} M\right)\right.\right.$, while $u_{\alpha}(\cdot+\sigma(\alpha), 2 \cdot-1)$ converges to $u$ in $C_{\mathrm{loc}}^{\infty}\left(\mathbb{R} \times[0,1], T^{*} M\right)$, for a suitable function $\sigma$ diverging at $+\infty$.

(ii) For every $u \in \mathcal{M}_{K}^{\Omega}\left(\gamma_{1}, \gamma_{2} ; x\right)$ there exists a unique connected component of $\mu_{\Upsilon}^{K}\left(\gamma_{1}, \gamma_{2} ; x\right)$ containing a curve $\alpha \mapsto\left(\alpha, u_{\alpha}\right)$ which converges to $(0, u)$.

Statement (i) can be proved by the standard gluing arguments in Floer theory. Here we prove statement (ii).

Given $u:\left[0,+\infty\left[\times[-1,1] \rightarrow T^{*} M\right.\right.$, we define the map

$$
\tilde{u}: \Sigma^{+}=\{z \in \mathbb{C} \mid \operatorname{Re} z \geq 0,0 \leq \operatorname{Im} z \leq 1\} \rightarrow T^{*} M^{2}, \quad \tilde{u}(z):=(\mathscr{C} u(\bar{z}), u(z)) .
$$

If we define $\tilde{x}:[0,1] \rightarrow T^{*} M^{2}$ and $\tilde{H} \in C^{\infty}\left([0,1] \times T^{*} M^{2}\right)$ by

$\tilde{x}(t):=(\mathscr{C} x((1-t) / 2), x((1+t) / 2)), \quad \tilde{H}\left(t, x_{1}, x_{2}\right):=H_{1}\left(1-t, \mathscr{C} x_{1}\right)+H_{2}\left(t, x_{2}\right)$,

we see that associating $\tilde{u}$ to $u$ produces a one-to-one correspondence between the space $\mathcal{M}_{K}^{\Omega}\left(\gamma_{1}, \gamma_{2} ; x\right)$ and the space $\widetilde{M}_{K}^{\Omega}\left(\gamma_{1}, \gamma_{2} ; x\right)$, which consists of the maps $\tilde{u}: \Sigma^{+} \rightarrow$ $T^{*} M^{2}$ solving $\bar{\partial}_{J}, \widetilde{H}(\widetilde{u})=0$ with boundary conditions

$$
\begin{array}{rlrl}
\tilde{u}(s) & \in N^{*} \Delta_{M}, & & \forall s \geq 0, \\
\tilde{u}(s+i) & \in T_{q_{0}}^{*} M \times T_{q_{0}}^{*} M, & & \forall s \geq 0, \\
\pi \circ \tilde{u}_{1}(0,1-\cdot) & \in W^{u}\left(\gamma_{1}\right), \quad \pi \circ \tilde{u}_{2}(0, \cdot) \in W^{u}\left(\gamma_{2}\right), & & \\
\lim _{s \rightarrow+\infty} \tilde{u}(s+i t)=\tilde{x}(t) . & &
\end{array}
$$

Similarly, we have a one-to-one correspondence between $\mu_{\Upsilon}^{K}\left(\gamma_{1}, \gamma_{2} ; x\right)$ and the space $\widetilde{M}_{\Upsilon}^{K}\left(\gamma_{1}, \gamma_{2} ; x\right)$ consisting of pairs $(\alpha, \widetilde{u})$ where $\alpha$ is a positive number and $\tilde{u}: \Sigma^{+} \rightarrow$ $T^{*} M^{2}$ is a solution of the problem above, with (6-25) replaced by

$$
\tilde{u}(s) \in T_{q_{0}}^{*} M \times T_{q_{0}}^{*} M, \quad \forall s \in[0, \alpha], \quad \tilde{u}(s) \in N^{*} \Delta_{M}, \quad \forall s \geq \alpha .
$$

Fix some $\widetilde{u}^{0} \in \widetilde{M}_{K}^{\Omega}\left(\gamma_{1}, \gamma_{2} ; x\right)$. Since we are looking for solutions near $\widetilde{u}^{0}$, we can localize the problem as follows. Let $k=i^{\Omega}\left(\gamma_{1} ; L_{1}\right)+i^{\Omega}\left(\gamma_{2} ; L_{2}\right)$. Let $q: \mathbb{R}^{k} \times \Sigma^{+} \rightarrow$ $M^{2}$ be a map such that $q(0, z)=\pi \circ \tilde{u}^{0}(z), \quad \forall z \in \Sigma^{+}, \quad q(\lambda, s+i t) \rightarrow \pi \circ \tilde{x}(t)$ for $s \rightarrow+\infty, \forall \lambda \in \mathbb{R}^{k}$, 
and such that the map

$$
\mathbb{R}^{k} \ni \lambda \mapsto\left(q_{1}(\lambda, 0,1-\cdot), q_{2}(\lambda, 0, \cdot) \in \Omega^{1}\left(M^{2}\right)\right.
$$

is a diffeomorphism onto a neighborhood of $\pi \circ\left(\tilde{x}_{1}(-\cdot), \tilde{x}_{2}\right)$ in $W^{u}\left(\gamma_{1}\right) \times W^{u}\left(\gamma_{2}\right)$. By means of a suitable trivialization of $q^{*}\left(T M^{2}\right)$ and using the usual $W^{1, p}$ Sobolev setting with $p>2$, we can transform the problem of finding $\tilde{u}$ solving $\bar{\partial}_{J}, \widetilde{H}(\widetilde{u})=0$ together with (6-26), (6-27) and (6-28) and being close to $\tilde{u}^{0}$, into the problem of finding pairs $(\lambda, u) \in \mathbb{R}^{k} \times W^{1, p}\left(\Sigma^{+}, T^{*} \mathbb{R}^{2 n}\right)$, solving an equation of the form

$$
\bar{\partial} u(z)+f(\lambda, z, u(z))=0, \quad \forall z \in \Sigma^{+},
$$

with boundary conditions

$$
u(i t) \in N^{*}(0), \quad \forall t \in[0,1], \quad u(s+i) \in N^{*}(0), \quad \forall s \geq 0 .
$$

Then the boundary condition $(6-25)$ is translated into

$$
u(s) \in N^{*} \Delta_{\mathbb{R}^{n}}, \quad \forall s \geq 0,
$$

and the solution $\tilde{u}^{0}$ corresponds to the solution $\lambda=0$ and $u \equiv 0$ of (6-30), (6-31), and (6-32). On the other hand, the problem $\widetilde{\mathcal{M}}_{\Upsilon}^{K}\left(\gamma_{1}, \gamma_{2} ; x\right)$ of finding $\left(\alpha, \widetilde{u}^{\alpha}\right)$ solving $\bar{\partial}_{J, \tilde{H}}\left(\widetilde{u}^{\alpha}\right)=0$ together with (6-26), (6-27), (6-28) and (6-29) corresponds to the problem of finding $(\lambda, u) \in \mathbb{R}^{k} \times W^{1, p}\left(\Sigma^{+}, T^{*} \mathbb{R}^{2 n}\right)$ solving (6-30) with boundary conditions (6-31) and

$$
u(s) \in N^{*}(0), \quad \forall s \in[0, \alpha], \quad u(s) \in N^{*} \Delta_{\mathbb{R}^{n}}, \quad \forall s \geq \alpha .
$$

In order to find a common functional setting, it is convenient to turn the boundary condition (6-33) into (6-32) by means of a suitable conformal change of variables on the half-strip $\Sigma^{+}$.

The holomorphic function $z \mapsto \cos z$ maps the half strip $\{0<\operatorname{Re} z<\pi, \operatorname{Im} z>0\}$ biholomorphically onto the upper half-plane $\mathbb{H}=\{\operatorname{Im} z>0\}$. It is also a homeomorphism between the closure of these domains. We denote by arccos the determination of the arc-cosine which is the inverse of this function. Then the function $z \mapsto(1+\cos (i \pi z)) / 2$ is a biholomorphism from the interior of $\Sigma^{+}$to $\mathbb{H}$, mapping 0 into 1 and $i$ into 0 . Let $\epsilon>0$. If we conjugate the linear automorphism $z \mapsto(1+\epsilon) z$ of $\mathbb{H}$ by the latter biholomorphism, we obtain the map

$$
\varphi_{\epsilon}(z)=\frac{i}{\pi} \arccos ((1+\epsilon) \cos (i \pi z)+\epsilon) .
$$

The map $\varphi_{\epsilon}$ is a homeomorphism of $\Sigma^{+}$onto itself, it is biholomorphic in the interior, it preserves the upper part of the boundary $i+\mathbb{R}^{+}$, while it slides the left part $i[0,1]$ 
and the lower part $\mathbb{R}^{+}$by moving the corner point 0 into the real positive number

$$
\alpha(\epsilon):=-\frac{i}{\pi} \arccos (1+2 \epsilon) .
$$

The function $\epsilon \mapsto \alpha(\epsilon)$ is invertible, and we denote by $\alpha \mapsto \epsilon(\alpha)$ its inverse. Moreover, $\varphi_{\epsilon}$ converges to the identity uniformly on compact subsets of $\Sigma^{+}$for $\epsilon \rightarrow 0$. An explicit computation shows that

$$
\varphi_{\epsilon}^{\prime}-1 \rightarrow 0 \quad \text { in } L^{p}\left(\Sigma^{+}\right), \quad \text { if } 1<p<4 .
$$

If $u: \Sigma^{+} \rightarrow T^{*} \mathbb{R}^{2 n}$ and $\alpha>0$, we define

$$
v(z):=u\left(\varphi_{\epsilon(\alpha)}(z)\right) .
$$

Since $\varphi_{\epsilon}$ is holomorphic, $\bar{\partial}\left(u \circ \varphi_{\epsilon}\right)=\varphi_{\epsilon}^{\prime} \cdot \bar{\partial} u \circ \varphi_{\epsilon}$. Therefore, $u$ solves the equation (6-30) if and only if $v$ solves the equation

$$
\bar{\partial} v(z)+\varphi_{\epsilon(\alpha)}^{\prime}(z) f\left(\lambda, \varphi_{\epsilon(\alpha)}(z), v(z)\right)=0 .
$$

Given $2<p<4$, we set

$$
\begin{aligned}
W_{*}^{1, p}\left(\Sigma^{+}, T^{*} \mathbb{R}^{2 n}\right)=\left\{v \in W^{1, p}\left(\Sigma^{+}, T^{*} \mathbb{R}^{2 n}\right) \mid v(s, 0) \in N^{*} \Delta_{\mathbb{R}^{n}} \forall s \geq 0,\right. \\
\left.v(s, 1) \in N^{*}(0) \forall s \geq 0, v(0, t) \in N^{*}(0) \forall t \in[0,1]\right\},
\end{aligned}
$$

and we consider the operator

$$
\begin{aligned}
& F:\left[0,+\infty\left[\times \mathbb{R}^{k} \times W_{*}^{1, p}\left(\Sigma^{+}, T^{*} \mathbb{R}^{2 n}\right) \rightarrow L^{p}\left(\Sigma^{+}, T^{*} \mathbb{R}^{2 n}\right),\right.\right. \\
& F(\alpha, \lambda, v)=\bar{\partial} v+\varphi_{\epsilon(\alpha)}^{\prime} f\left(\lambda, \varphi_{\epsilon(\alpha)}(\cdot), v\right),
\end{aligned}
$$

where $\varphi_{0}=$ id. The problem of finding $(\alpha, \tilde{u})$ in $\widetilde{M}_{\Upsilon}^{K}\left(\gamma_{1}, \gamma_{2} ; x\right)$ with $\tilde{u}$ close to $\tilde{u}^{0}$ is equivalent to finding zeroes of the operator $F$ of the form $(\alpha, \lambda, v)$ with $\alpha>0$. By (6-34), the operator $F$ is continuous, and its differential $D_{(\lambda, v)} F$ with respect to the variables $(\lambda, v)$ is continuous. The transversality assumption that $\widetilde{u}^{0}$ is a nondegenerate solution of problem $\widetilde{M}_{K}^{\Omega}\left(\gamma_{1}, \gamma_{2} ; x\right)$ is translated into the fact that $D_{(\lambda, v)} F(0,0,0)$ is an isomorphism. Then the parametric inverse mapping theorem implies that there is a unique curve $\alpha \mapsto(\lambda(\alpha), v(\alpha)), 0<\alpha<\alpha_{0}$, converging to $(0,0)$ for $\alpha \rightarrow 0$, and such that $(\lambda(\alpha), v(\alpha))$ is the unique zero of $F(\alpha, \cdot, \cdot)$ in a neighborhood of $(0,0)$. This concludes the proof of statement (ii).

\subsection{Proof of Proposition 4.7}

The setting We recall the setting of Section 4.4. Let $\gamma_{1} \in \mathscr{P} \Lambda\left(L_{1}\right), \gamma_{2} \in \mathscr{P} \Lambda\left(L_{2}\right)$, and $x \in \mathscr{P}^{\Theta}\left(H_{1} \oplus H_{2}\right)$. If $\alpha \geq 0$, then $\mu_{\alpha}^{K}\left(\gamma_{1}, \gamma_{2} ; x\right)$ is the space of solutions 
$u: \Sigma^{+}=\{z \in \mathbb{C} \mid \operatorname{Re} z \geq 0,0 \leq \operatorname{Im} z \leq 1\} \rightarrow T^{*} M^{2}$ of the equation

$$
\bar{\partial}_{J, H_{1} \oplus H_{2}}(u)=0,
$$

satisfying the boundary conditions

$$
\begin{array}{ccc}
\pi \circ u(\cdot i) \in W^{u}\left(\left(\gamma_{1}, \gamma_{2}\right) ; X_{L_{1} \oplus L_{2}}^{\Lambda}\right), & \\
(u(s), \mathscr{b} u(s+i)) \in N^{*} \Delta_{M^{2}} & \text { if } 0 \leq s<\alpha, \\
(u(s), \mathscr{b} u(s+i)) \in N^{*} \Delta_{M}^{(4)} & \text { if } s \geq \alpha, \\
\lim _{s \rightarrow+\infty} u(s+t i)=x(t) . &
\end{array}
$$

The energy of a solution $u \in \mathcal{M}_{\alpha}^{K}\left(\gamma_{1}, \gamma_{2} ; x\right)$ is uniformly bounded:

$$
E(u):=\int_{\Sigma^{+}}\left|\partial_{s} u\right|^{2} d s d t \leq \mathbb{S}_{L_{1}}\left(\gamma_{1}\right)+\mathbb{S}_{L_{2}}\left(\gamma_{2}\right)-\mathbb{A}_{H_{1} \oplus H_{2}}(x) .
$$

Let $\alpha_{0}>0$. For a generic choice of $L_{1}, L_{2}$, and $X_{L_{1} \oplus L_{2}}^{\Lambda}$, both $\mu_{0}^{K}\left(\gamma_{1}, \gamma_{2} ; x\right)$ and $\mu_{\alpha_{0}}^{K}\left(\gamma_{1}, \gamma_{2} ; x\right)$ are smooth oriented manifolds of dimension

$$
i^{\Lambda}\left(\gamma_{1}, L_{1}\right)+i^{\Lambda}\left(\gamma_{2}, L_{2}\right)-\mu^{\Theta}(x)-n,
$$

for every $\gamma_{1} \in \mathscr{P}^{\Lambda}\left(L_{1}\right), \gamma_{2} \in \mathscr{P}^{\Lambda}\left(L_{2}\right), x \in \mathscr{P}^{\Theta}\left(H_{1} \oplus H_{2}\right)$ (see Proposition 4.5). The usual counting process defines the chain maps

$$
K_{0}^{\Lambda}, K_{\alpha_{0}}^{\Lambda}:\left(M\left(\mathbb{S}_{L_{1}}^{\Lambda}\right) \otimes M\left(\mathbb{S}_{L_{2}}^{\Lambda}\right)\right)_{*} \longrightarrow F_{*-n}^{\Theta}\left(H_{1} \oplus H_{2}\right),
$$

and we wish to prove that $K_{0}^{\Lambda} \otimes K_{\alpha_{0}}^{\Lambda}$ is chain homotopic to $K_{\alpha_{0}}^{\Lambda} \otimes K_{0}^{\Lambda}$. Since $K_{\alpha_{0}}^{\Lambda}$ is chain homotopic to $K_{\alpha_{1}}^{\Lambda}$ for $\left.\alpha_{0}, \alpha_{1} \in\right] 0,+\infty$, we may as well assume that $\alpha_{0}$ is small. Moreover, since the chain maps $K_{0}^{\Lambda}$ and $K_{\alpha_{0}}^{\Lambda}$ preserve the filtrations of the Morse and Floer complexes given by the action sublevels

$$
\mathbb{S}_{L_{1}}^{\Lambda}\left(\gamma_{1}\right)+\mathbb{S}_{L_{2}}^{\Lambda}\left(\gamma_{2}\right) \leq A, \quad \mathbb{A}_{H_{1} \oplus H_{2}}(x) \leq A,
$$

we can work with the subcomplexes corresponding to a fixed (but arbitrary) action bound $A$. We also fix our generic data in such a way that transversality holds for the problem $\mu_{0}^{K}$.

The union of all the spaces of solutions $\mu_{0}^{K}\left(\gamma_{1}, \gamma_{2} ; x\right)$ where $\gamma_{1} \in \mathscr{P}^{\Lambda}\left(L_{1}\right), \gamma_{2} \in$ $\mathscr{P}^{\Lambda}\left(L_{2}\right)$, and $x \in \mathscr{P}^{\Theta}\left(H_{1} \oplus H_{2}\right)$ satisfy the index identity

$$
i^{\Lambda}\left(\gamma_{1}\right)+i^{\Lambda}\left(\gamma_{2}\right)-\mu^{\Theta}(x)=n,
$$

and the action estimates

$$
\mathbb{S}_{L_{1}}\left(\gamma_{1}\right)+\mathbb{S}_{L_{2}}\left(\gamma_{2}\right) \leq A, \quad \mathbb{A}_{H_{1} \oplus H_{2}}(x) \leq A,
$$


is a compact zero-dimensional manifold, hence a finite set. We denote by 2 the finite subset of $M$ consisting of the points $q \in M$ such that $\pi \circ u(0)=\pi \circ u(i)=(q, q)$ for some $u$ in the above finite set. We fix a positive number $\delta$ such that for every $q \in 2$ the ball $B_{\delta}(q)$ is diffeomorphic to $\mathbb{R}^{n}$, and

$$
B_{\delta}(q) \cap B_{\delta}\left(q^{\prime}\right)=\varnothing, \quad \forall q, q^{\prime} \in \mathcal{2}, q \neq q^{\prime} .
$$

Since real Grassmannians of a given dimension are connected, we can find a smooth path of $3 n$-dimensional linear subspaces of $\Delta_{\mathbb{R}^{2 n}} \times \Delta_{\mathbb{R}^{2 n}} \subset \mathbb{R}^{8 n}$ connecting

$$
\Delta_{\mathbb{R}^{n}}^{(4)} \times \Delta_{\mathbb{R}^{2 n}} \text { to } \Delta_{\mathbb{R}^{2 n}} \times \Delta_{\mathbb{R}^{n}}^{(4)},
$$

and containing the intersection of these two spaces, that is $\Delta_{\mathbb{R}^{n}}^{(4)} \times \Delta_{\mathbb{R}^{n}}^{(4)}$. Therefore, for every pair of points $q, q^{\prime} \in 2$ we can find a smooth isotopy of embeddings

$$
\varphi:[0,1] \times \mathbb{R}^{3 n} \rightarrow B_{\delta}(q)^{4} \times B_{\delta}\left(q^{\prime}\right)^{4} \subset M^{8},
$$

such that, setting $V_{q, q^{\prime}}^{\lambda}:=\varphi\left(\{\lambda\} \times \mathbb{R}^{3 n}\right)$, we have:

(i) $V_{q, q^{\prime}}^{\lambda}$ is relatively closed in $B_{\delta}(q)^{4} \times B_{\delta}\left(q^{\prime}\right)^{4}$.

(ii) $\left(\Delta_{M}^{(4)} \times \Delta_{M}^{(4)}\right) \cap\left(B_{\delta}(q)^{4} \times B_{\delta}\left(q^{\prime}\right)^{4}\right) \subset V_{q, q^{\prime}}^{\lambda}$ for every $\lambda \in[0,1]$.

(iii) $V_{q, q^{\prime}}^{\lambda} \subset \Delta_{M^{2}} \times \Delta_{M^{2}}$ for every $\lambda \in[0,1]$.

(iv) $V_{q, q^{\prime}}^{0}=\left(\Delta_{M}^{(4)} \times \Delta_{M^{2}}\right) \cap\left(B_{\delta}(q)^{4} \times B_{\delta}\left(q^{\prime}\right)^{4}\right)$.

(v) $V_{q, q^{\prime}}^{1}=\left(\Delta_{M^{2}} \times \Delta_{M}^{(4)}\right) \cap\left(B_{\delta}(q)^{4} \times B_{\delta}\left(q^{\prime}\right)^{4}\right)$.

We define $V^{\lambda} \subset M^{8}$ to be the union of $V_{q, q^{\prime}}^{\lambda}$ over all pairs $\left(q, q^{\prime}\right) \in 2 \times 2$. By (6-42), $V^{\lambda}$ is an isotopy of (noncompact) $3 n$-dimensional submanifolds of $M^{8}$.

Let $\gamma_{1}, \gamma_{3} \in \mathscr{P}^{\Lambda}\left(L_{1}\right), \gamma_{2}, \gamma_{4} \in \mathscr{P}^{\Lambda}\left(L_{2}\right)$, and $x_{1}, x_{2} \in \mathscr{P}^{\Theta}\left(H_{1} \oplus H_{2}\right)$ satisfy the index identity

$$
i^{\Lambda}\left(\gamma_{1}\right)+i^{\Lambda}\left(\gamma_{2}\right)+i^{\Lambda}\left(\gamma_{3}\right)+i^{\Lambda}\left(\gamma_{4}\right)-\mu^{\Theta}\left(x_{1}\right)-\mu^{\Theta}\left(x_{2}\right)=2 n,
$$

and the action bounds

$$
\begin{array}{r}
\mathbb{S}_{L_{1}}\left(\gamma_{1}\right)+\mathbb{S}_{L_{2}}\left(\gamma_{2}\right)+\mathbb{S}_{L_{1}}\left(\gamma_{3}\right)+\mathbb{S}_{L_{2}}\left(\gamma_{4}\right) \leq A, \\
\mathbb{A}_{H_{1} \oplus H_{2}}\left(x_{1}\right)+\mathbb{A}_{H_{1} \oplus H_{2}}\left(x_{2}\right) \leq A .
\end{array}
$$

Given $\alpha>0$, we define

$$
\mu_{\alpha}^{P}\left(\gamma_{1}, \gamma_{2}, \gamma_{3}, \gamma_{4} ; x_{1}, x_{2}\right)
$$

to be the set of pairs $(\lambda, u)$ where $\lambda \in[0,1]$ and $u:\left[0,+\infty\left[\times[0,1] \rightarrow T^{*} M^{4}\right.\right.$ is a solution of the equation

$$
\bar{\partial}_{J, H_{1} \oplus H_{2} \oplus H_{1} \oplus H_{2}}(u)=0,
$$


satisfying the boundary conditions

$$
\begin{array}{clrl}
\pi \circ u(0, \cdot) \in W^{u}\left(\gamma_{1}\right) \times W^{u}\left(\gamma_{2}\right) \times W^{u}\left(\gamma_{3}\right) \times W^{u}\left(\gamma_{4}\right), & \\
(u(s), \mathscr{C} u(s+i)) \in N^{*} V^{\lambda} & & \text { if } 0 \leq s \leq \alpha, \\
(u(s), \mathscr{b} u(s+i)) \in N^{*}\left(\Delta_{M}^{(4)} \times \Delta_{M}^{(4)}\right) & & \text { if } s \geq \alpha, \\
\lim _{s \rightarrow+\infty} u(s+t i)=\left(x_{1}(t), x_{2}(t)\right) . &
\end{array}
$$

By property (ii) above, the submanifold $V^{\lambda}$ is partially orthogonal to $\Delta_{M}^{(4)} \times \Delta_{M}^{(4)}$ for every $\lambda \in[0,1]$, so the results of Section 5 provide us with elliptic estimates for solutions of the above problem.

Notice that if $(0, u)$ belongs to $\mu_{\alpha}^{P}\left(\gamma_{1}, \gamma_{2}, \gamma_{3}, \gamma_{4} ; x_{1}, x_{2}\right)$, then writing $u=\left(u_{1}, u_{2}\right)$ where $u_{1}$ and $u_{2}$ take values into $T^{*} M^{2}$, we have

$$
u_{1} \in M_{0}^{K}\left(\gamma_{1}, \gamma_{2} ; x_{1}\right), \quad u_{2} \in \mathcal{M}_{\alpha}^{K}\left(\gamma_{3}, \gamma_{4} ; x_{2}\right) .
$$

If transversality holds, we deduce the index estimates

$$
i^{\Lambda}\left(\gamma_{1}\right)+i^{\Lambda}\left(\gamma_{2}\right)-\mu^{\Theta}\left(x_{1}\right) \geq n, \quad i^{\Lambda}\left(\gamma_{3}\right)+i^{\Lambda}\left(\gamma_{4}\right)-\mu^{\Theta}\left(x_{2}\right) \geq n .
$$

But then (6-43) implies that the above inequalities are indeed identities. Similarly, if $(1, u)$ belongs to $\mu_{\alpha}^{P}\left(\gamma_{1}, \gamma_{2}, \gamma_{3}, \gamma_{4} ; x_{1}, x_{2}\right)$, we deduce that

$$
u_{1} \in M_{\alpha}^{K}\left(\gamma_{1}, \gamma_{2} ; x_{1}\right), \quad u_{2} \in M_{0}^{K}\left(\gamma_{3}, \gamma_{4} ; x_{2}\right)
$$

and

$$
i^{\Lambda}\left(\gamma_{1}\right)+i^{\Lambda}\left(\gamma_{2}\right)-\mu^{\Theta}\left(x_{1}\right)=n, \quad i^{\Lambda}\left(\gamma_{3}\right)+i^{\Lambda}\left(\gamma_{4}\right)-\mu^{\Theta}\left(x_{2}\right)=n .
$$

Conversely, we would like to show that pairs of solutions in $\mu_{0}^{K} \times \mu_{\alpha}^{K}$ (or $\mu_{\alpha}^{K} \times \mu_{0}^{K}$ ) correspond to elements of $\mu_{\alpha}^{P}$ of the form $(0, u)$ (or $(1, u)$ ), at least if $\alpha$ is small. This fact follows from the following localization result:

Lemma 6.7 There exists a positive number $\alpha(A)$ such that for every $\alpha \in] 0, \alpha(A)]$, for every $\gamma_{1}, \gamma_{3} \in \mathscr{P}^{\Lambda}\left(L_{1}\right), \gamma_{2}, \gamma_{4} \in \mathscr{P}^{\Lambda}\left(L_{2}\right), x_{1}, x_{2} \in \mathscr{P}^{\Theta}\left(H_{1} \oplus H_{2}\right)$ satisfying (6-43) and (6-44) and for every $(\lambda, u) \in \mathcal{M}_{\alpha}^{P}\left(\gamma_{1}, \gamma_{2}, \gamma_{3}, \gamma_{4} ; x_{1}, x_{2}\right)$ there holds

$$
\pi \circ u([0, \alpha])=\pi \circ u([0, \alpha]+i) \subset B_{\delta / 2}(q)^{2} \times B_{\delta / 2}\left(q^{\prime}\right)^{2},
$$

for suitable $q, q^{\prime} \in 2$.

Proof By contradiction, we assume that there are an infinitesimal sequence of positive numbers $\left(\alpha_{h}\right)$ and elements $\left(\lambda_{h}, u_{h}\right) \in \mathcal{M}_{\alpha_{h}}^{P}\left(\gamma_{1}, \gamma_{2}, \gamma_{3}, \gamma_{4} ; x_{1}, x_{2}\right)$ where $\gamma_{1}, \gamma_{2}, \gamma_{3}$, $\gamma_{4}, x_{1}, x_{2}$ satisfy (6-43), (6-44) and

$$
\pi \circ u_{h}\left(\left[0, \alpha_{h}\right]\right)=\pi \circ u_{h}\left(\left[0, \alpha_{h}\right]+i\right) \not \subset
$$$$
\bigcup B_{\delta / 2}(q)^{2} \times B_{\delta / 2}\left(q^{\prime}\right)^{2}
$$ 
Claim 1 Up to a subsequence, $c_{h}(t):=\pi \circ u_{h}(0, t)$ converges to some $c$ in the space $W^{1,2}\left([0,1], M^{4}\right)$ such that $c(0)=c(1)=\left(q, q, q^{\prime}, q^{\prime}\right)$ with $\left(q, q^{\prime}\right) \in 2 \times 2$, and $u_{h}$ converges to some $u \in M_{0}^{K}\left(\gamma_{1}, \gamma_{2} ; x_{1}\right) \times \mu_{0}^{K}\left(\gamma_{3}, \gamma_{4} ; x_{2}\right)$ such that $\pi \circ u(t i)=c(t)$ in $C_{\mathrm{loc}}^{\infty}(] 0,+\infty\left[\times[0,1], T^{*} M^{4}\right)$ and uniformly on compact subsets of $[0,+\infty[\times[0,1] \backslash$ $\{(0,0),(0,1)\}$.

By (6-46), $c_{h}$ is an element of $W^{u}\left(\gamma_{1}\right) \times W^{u}\left(\gamma_{2}\right) \times W^{u}\left(\gamma_{3}\right) \times W^{u}\left(\gamma_{4}\right)$. The latter space is precompact in $W^{1,2}\left([0,1], M^{4}\right)$. By the argument of breaking gradient flow lines, up to a subsequence we may assume that $\left(c_{h}\right)$ converges in $W^{1,2}$ to a curve $c$ belonging to $W^{u}\left(\tilde{\gamma}_{1}\right) \times W^{u}\left(\tilde{\gamma}_{2}\right) \times W^{u}\left(\tilde{\gamma}_{3}\right) \times W^{u}\left(\tilde{\gamma}_{4}\right)$, for some $\tilde{\gamma}_{1}, \tilde{\gamma}_{3} \in \mathscr{P}^{\Lambda}\left(L_{1}\right)$, $\tilde{\gamma}_{2}, \tilde{\gamma}_{4} \in \mathscr{P}^{\Lambda}\left(L_{2}\right)$ such that

(6-51) either $\sum_{j=1}^{4} i^{\Lambda}\left(\tilde{\gamma}_{j}\right)<\sum_{j=1}^{4} i^{\Lambda}\left(\gamma_{j}\right)$ or $\quad\left(\tilde{\gamma}_{1}, \tilde{\gamma}_{2}, \tilde{\gamma}_{3}, \tilde{\gamma}_{4}\right)=\left(\gamma_{1}, \gamma_{2}, \gamma_{3}, \gamma_{4}\right)$.

Similarly, the upper bound (6-41) on the energy $E\left(u_{h}\right)$ implies that $\left(u_{h}\right)$ converges to some $u$ in $C_{\text {loc }}^{\infty}(] 0,+\infty[\times[0,1])$, using the $L^{\infty}$ estimates of Section 6 , the standard argument excluding bubbling off of spheres and disks, and elliptic bootstrap. The same arguments, together with the $W^{1,2}$ convergence of $\left(c_{h}\right)$ to $c$, imply that $\left(u_{h}\right)$ converges to $u$ uniformly on compact subsets of $[0,+\infty[\times[0,1] \backslash\{(0,0),(0,1)\}$ (actually, the $W^{1,2}$ convergence of the boundary data implies $W^{3 / 2,2}$ convergence near the portion of the boundary $\{0\} \times] 0,1[$ ). In particular,

$$
\begin{gathered}
(u(s), \mathscr{C} u(s+i)) \in N^{*}\left(\Delta_{M}^{(4)} \times \Delta_{M}^{(4)}\right) \quad \forall s>0, \\
\pi \circ u(0, t)=\pi \circ u(1, t)=c(t) \quad \forall t \in] 0,1[.
\end{gathered}
$$

The limit $u$ satisfies Equation (6-45), and

$$
\lim _{s \rightarrow+\infty} u(s+t i)=\left(\tilde{x}_{1}(t), \tilde{x}_{2}(t)\right),
$$

with $\left(\tilde{x}_{1}, \tilde{x}_{2}\right) \in \mathscr{P P}^{\Theta}\left(H_{1} \oplus H_{2}\right) \times \mathscr{P}^{\Theta}\left(H_{1} \oplus H_{2}\right)$ such that

(6-54) either $\mu^{\Theta}\left(\tilde{x}_{1}\right)+\mu^{\Theta}\left(\tilde{x}_{2}\right)>\mu^{\Theta}\left(x_{1}\right)+\mu^{\Theta}\left(x_{2}\right)$ or $\left(\tilde{x}_{1}, \tilde{x}_{2}\right)=\left(x_{1}, x_{2}\right)$,

by the argument of breaking Floer trajectories. Due to finite energy,

$$
\begin{aligned}
E(u) & \leq \liminf _{h \rightarrow+\infty} E\left(u_{h}\right) \\
& \leq \mathbb{S}_{L_{1}}\left(\gamma_{1}\right)+\mathbb{S}_{L_{2}}\left(\gamma_{2}\right)+\mathbb{S}_{L_{1}}\left(\gamma_{3}\right)+\mathbb{S}_{L_{2}}\left(\gamma_{4}\right)-\mathbb{A}_{H_{1} \oplus H_{2}}\left(x_{1}\right)-\mathbb{A}_{H_{1} \oplus H_{2}}\left(x_{2}\right),
\end{aligned}
$$

we find by removal singularities (Proposition 6.4) a continuous extension of $u$ to the corner points 0 and $i$. By (6-52) and (6-53) we have

$$
(u(0), \mathscr{b} u(i)) \in N^{*}\left(\Delta_{M}^{(4)} \times \Delta_{M}^{(4)}\right), \quad \pi \circ u(0)=\pi \circ u(i)=c(0)=c(1) .
$$


It follows that, setting $c=\left(c_{1}, c_{2}, c_{3}, c_{4}\right)$, there holds $c_{1}(0)=c_{1}(1)=c_{2}(0)=c_{2}(1)$ and $c_{3}(0)=c_{3}(1)=c_{4}(0)=c_{4}(1)$. So $\left(c_{1}, c_{2}\right)$ and $\left(c_{3}, c_{4}\right)$ describe two figure-8 loops, and $u$ belongs to $\mu_{0}^{K}\left(\tilde{\gamma}_{1}, \tilde{\gamma}_{2} ; \tilde{x}_{1}\right) \times \mu_{0}^{K}\left(\tilde{\gamma}_{3}, \tilde{\gamma}_{4} ; \tilde{x}_{2}\right)$. In particular, the latter space in not empty, so

$$
i^{\Lambda}\left(\tilde{\gamma}_{1}\right)+i^{\Lambda}\left(\tilde{\gamma}_{2}\right)-\mu^{\Theta}\left(\tilde{x}_{1}\right) \geq n, \quad i^{\Lambda}\left(\tilde{\gamma}_{3}\right)+i^{\Lambda}\left(\tilde{\gamma}_{4}\right)-\mu^{\Theta}\left(\tilde{x}_{2}\right) \geq n .
$$

Together with (6-43), this implies

$$
\sum_{j=1}^{4} i^{\Lambda}\left(\tilde{\gamma}_{j}\right)-\sum_{j=1}^{2} \mu^{\Theta}\left(\tilde{x}_{j}\right) \geq 2 n=\sum_{j=1}^{4} i^{\Lambda}\left(\gamma_{j}\right)-\sum_{j=1}^{2} \mu^{\Theta}\left(x_{j}\right) .
$$

Comparing the above inequality with (6-51) and (6-54), we deduce that

$$
\left(\tilde{\gamma}_{1}, \tilde{\gamma}_{2}, \tilde{\gamma}_{3}, \tilde{\gamma}_{4}\right)=\left(\gamma_{1}, \gamma_{2}, \gamma_{3}, \gamma_{4}\right), \quad\left(\tilde{x}_{1}, \tilde{x}_{2}\right)=\left(x_{1}, x_{2}\right) .
$$

Therefore, $u$ belongs to $\mu_{0}^{K}\left(\gamma_{1}, \gamma_{2} ; x_{1}\right) \times \mu_{0}^{K}\left(\gamma_{3}, \gamma_{4} ; x_{2}\right)$ and

$$
c(0)=c(1)=\left(q, q, q^{\prime}, q^{\prime}\right),
$$

for some pair $\left(q, q^{\prime}\right) \in 2 \times 2$, concluding the proof of Claim 1 .

Claim 2 Up to a subsequence, the sequences of maps

$$
v_{h}^{0}(z):=u_{h}\left(\alpha_{h} z\right), \quad v_{h}^{1}(z):=u_{h}\left(i+\alpha_{h} \bar{z}\right),
$$

converge to constant maps $v^{0}, v^{1}$ uniformly on compact subsets of $[0,+\infty[\times[0,+\infty[$. Moreover, $\pi\left(v^{0}\right)=\pi\left(v^{1}\right)=\left(q, q, q^{\prime}, q^{\prime}\right)$ for some pair $\left(q, q^{\prime}\right) \in 2 \times 2$.

It is convenient to replace the nonlocal boundary conditions (6-47), (6-48) by local ones. We define the sequence of maps

$$
w_{h}:\left[0,+\infty\left[\times\left[0,1 / \alpha_{h}\right] \rightarrow T^{*} M^{8}, \quad w_{h}(z):=\left(v_{h}^{0}(z), \mathscr{C} v_{h}^{1}(z)\right) .\right.\right.
$$

Then $w_{h}$ satisfies the local boundary conditions

$$
\begin{aligned}
& w_{h}(s)=\left(u_{h}\left(\alpha_{h} s\right), \mathscr{C} u_{h}\left(\alpha_{h} s+i\right)\right) \in \begin{cases}N^{*} V^{\lambda_{h}} & \text { if } 0 \leq s \leq 1, \\
N^{*}\left(\Delta_{M}^{(4)} \times \Delta_{M}^{(4)}\right) & \text { if } s \geq 1,\end{cases} \\
& \text { (6-56) } \pi \circ w_{h}(t i)=\left(\pi \circ u_{h}\left(0, \alpha_{h} t\right), \pi \circ u_{h}\left(0,\left(1-\alpha_{h}\right) t\right)\right)=\left(c_{h}\left(\alpha_{h} t\right), c_{h}\left(1-\alpha_{h} t\right)\right) \text {. }
\end{aligned}
$$

Up to a subsequence, we may assume that $\left(\lambda_{h}\right)$ converges to some $\lambda \in[0,1]$. The maps $w_{h}$ solve a Floer equation of the form

$$
\bar{\partial}_{J}\left(w_{h}\right)=\alpha_{h} J X_{K},
$$


for a suitable time-dependent Hamiltonian $K$ on $T^{*} M^{8}$. Since we have applied a conformal rescaling, the energy of $w_{h}$ is uniformly bounded, so $\left(w_{h}\right)$ converges up to a subsequence to some $J$-holomorphic map $w$ : $\left[0,+\infty\left[\times\left[0,+\infty\left[\rightarrow T^{*} M^{8}\right.\right.\right.\right.$ uniformly on compact subsets of $\left[0,+\infty\left[\times\left[0,+\infty\left[\right.\right.\right.\right.$. More precisely, we have $C_{\mathrm{loc}}^{\infty}(] 0,+\infty[\times[0,+\infty[)$ convergence once the domain $[0,+\infty[\times[0,+\infty[$ is transformed by a conformal mapping turning the portion near the boundary point $(1,0)$ into a neighborhood of $(0,0)$ in the upper-right quarter $\left.\mathbb{H}^{+}=\right] 0,+\infty[\times] 0,+\infty\left[\right.$, and we have $W^{3 / 2,2}$ convergence near the piece of the boundary $\{0\} \times] 0,+\infty[$. The $J$-holomorphic map $w$ has finite energy, so by removal singularities it has a continuous extension at $\infty$ (again, by Proposition 6.4 together with a suitable conformal change of variables). By (6-55) and (6-56) it satisfies the boundary conditions

$$
\begin{gathered}
w(s) \in \begin{cases}N^{*} V^{\lambda} & \text { if } 0 \leq s \leq 1, \\
N^{*}\left(\Delta_{M}^{(4)} \times \Delta_{M}^{(4)}\right) & \text { if } s \geq 1,\end{cases} \\
\pi \circ w(0, t)=(c(0), c(1))=(c(0), c(1)) \text { for } t \geq 0 .
\end{gathered}
$$

Since the boundary conditions are of conormal type and the Liouville one-form $\eta$ vanishes on conormals, we have

$\int_{\mathbb{H}^{+}}|\nabla w|^{2} d s d t=\int_{\mathbb{H}^{+}} w^{*}(\omega)=\int_{\mathbb{H}^{+}} w^{*}(d \eta)=\int_{\mathbb{H}^{+}} d w^{*}(\eta)=\int_{\partial \mathbb{H}^{+}} w^{*}(\eta)=0$, so $w$ is constant. By (6-57) and (6-58), $w$ belongs to $N^{*} V^{\lambda} \cap N^{*}\left(\Delta_{M}^{(4)} \times \Delta_{M}^{(4)}\right) \cap$ $T_{(c(0), c(0))}^{*} M^{8}$. In particular, $\pi(w)=(c(0), c(0))$, and since $c(0)=\left(q, q, q^{\prime}, q^{\prime}\right)$ with $\left(q, q^{\prime}\right) \in 2$, so Claim 2 follows.

Claim 2 contradicts (6-50), proving the lemma.

Remark 6.8 If $\left(u_{h}\right)$ is the sequence of solutions considered in the proof of the above lemma, we cannot conclude that $\left(u_{h}\right)$ converges uniformly in a neighborhood of the corner points $(0,0)$ and $(0,1)$. Indeed, generically this will not happen: otherwise the limit $u$ would be an element of $\mu_{0}^{K}\left(\gamma_{1}, \gamma_{2} ; x_{1}\right) \times \mu_{0}^{K}\left(\gamma_{3}, \gamma_{4} ; x_{2}\right)$ satisfying the extra condition $u(0)=u(i)$, and this is a problem of Fredholm index $-2 n$, for which generically there are no solutions.

We can now built the required chain homotopy between $K_{0}^{\Lambda} \otimes K_{\alpha_{0}}^{\Lambda}$ and $K_{\alpha_{0}}^{\Lambda} \otimes K_{0}^{\Lambda}$. We fix some $\left.\left.\alpha_{0} \in\right] 0, \alpha(A)\right]$, and we choose the generic data $L_{1}, L_{2}, X_{L_{1}}^{\Lambda}, X_{L_{2}}^{\Lambda}$ in such a way that transversality holds for the problems $\mu_{0}^{K}, \mu_{\alpha_{0}}^{K}$, and $\mu_{\alpha_{0}}^{P}$, and such that there are no elements of the form $(0, u)$ or $(1, u)$ in the zero-dimensional components of $\mu_{\alpha_{0}}^{P}$. 
Let $\gamma_{1}, \gamma_{3} \in \mathscr{P}^{\Lambda}\left(L_{1}\right), \gamma_{2}, \gamma_{4} \in \mathscr{P}^{\Lambda}\left(L_{2}\right), x_{1}, x_{2} \in \mathscr{P}^{\Theta}\left(H_{1} \oplus H_{2}\right)$ be Hamiltonian orbits satisfying the index identity (6-43) and the action bounds (6-44). Consider the one-dimensional manifold $\mu_{\alpha_{0}}^{P}\left(\gamma_{1}, \gamma_{2}, \gamma_{3}, \gamma_{4} ; x_{1}, x_{2}\right)$, and let

$$
\bar{M}_{\alpha_{0}}^{P}\left(\gamma_{1}, \gamma_{2}, \gamma_{3}, \gamma_{4} ; x_{1}, x_{2}\right)
$$

be the one-dimensional manifold with boundary obtained by attaching in the usual way elements $(\lambda, u)$ of the zero-dimensional spaces of solutions $\mu_{\alpha_{0}}^{P}$ (necessarily, with $0<\lambda<1$ ). The submanifold $V^{\lambda}$ is not compact, but its intersection with the closure of $B_{\delta / 2}(q)^{4} \times B_{\delta / 2}\left(q^{\prime}\right)^{4}, q, q^{\prime} \in 2$, is compact, so the localization Lemma 6.7 implies that $\bar{M}_{\alpha_{0}}^{P}\left(\gamma_{1}, \gamma_{2}, \gamma_{3}, \gamma_{4} ; x_{1}, x_{2}\right)$ is compact, and its intersections with $\{\lambda=0\}$ and $\{\lambda=1\}$ are

(6-59) $\mu_{\alpha_{0}}^{P}\left(\gamma_{1}, \gamma_{2}, \gamma_{3}, \gamma_{4} ; x_{1}, x_{2}\right) \cap\{\lambda=0\}=\mu_{0}^{K}\left(\gamma_{1}, \gamma_{2} ; x_{1}\right) \times \mu_{\alpha_{0}}^{K}\left(\gamma_{3}, \gamma_{4} ; x_{2}\right)$,

(6-60) $\mu_{\alpha_{0}}^{P}\left(\gamma_{1}, \gamma_{2}, \gamma_{3}, \gamma_{4} ; x_{1}, x_{2}\right) \cap\{\lambda=1\}=\mu_{\alpha_{0}}^{K}\left(\gamma_{1}, \gamma_{2} ; x_{1}\right) \times \mu_{0}^{K}\left(\gamma_{3}, \gamma_{4} ; x_{2}\right)$.

We denote by $M^{A}$ the subcomplex of

$$
M\left(\mathbb{S}_{L_{1}}^{\Lambda}\right) \otimes M\left(\mathbb{S}_{L_{2}}^{\Lambda}\right) \otimes M\left(\mathbb{S}_{L_{1}}^{\Lambda}\right) \otimes M\left(\mathbb{S}_{L_{2}}^{\Lambda}\right)
$$

spanned by generators $\gamma_{1} \otimes \gamma_{2} \otimes \gamma_{3} \otimes \gamma_{4}$ with

$$
\mathbb{S}_{L_{1}}\left(\gamma_{1}\right)+\mathbb{S}_{L_{2}}\left(\gamma_{2}\right)+\mathbb{S}_{L_{1}}\left(\gamma_{3}\right)+\mathbb{S}_{L_{2}}\left(\gamma_{4}\right) \leq A \text {. }
$$

Similarly, we denote by $F^{A}$ the subcomplex of

$$
F^{\Theta}\left(H_{1} \oplus H_{2}\right) \otimes F^{\Theta}\left(H_{1} \oplus H_{2}\right)
$$

spanned by generators $x_{1} \otimes x_{2}$ with

$$
\mathbb{A}_{H_{1} \oplus H_{2}}\left(x_{1}\right)+\mathbb{A}_{H_{1} \oplus H_{2}}\left(x_{2}\right) \leq A .
$$

We define a homomorphism

$$
P: M_{*}^{A} \rightarrow F_{*-2 n+1}^{A}
$$

by counting the elements of $\mu_{\alpha_{0}}^{P}\left(\gamma_{1}, \gamma_{2}, \gamma_{3}, \gamma_{4} ; x_{1}, x_{2}\right)$ in the zero-dimensional case:

$$
i^{\Lambda}\left(\gamma_{1}\right)+i^{\Lambda}\left(\gamma_{2}\right)+i^{\Lambda}\left(\gamma_{3}\right)+i^{\Lambda}\left(\gamma_{4}\right)-\mu^{\Theta}\left(x_{1}\right)-\mu^{\Theta}\left(x_{2}\right)=2 n-1 .
$$

Using the identities (6-59) and (6-60) we see that $P$ is a chain homotopy between the restrictions of $K_{0}^{\Lambda} \otimes K_{\alpha_{0}}^{\Lambda}$ and $K_{\alpha_{0}}^{\Lambda} \otimes K_{\alpha}^{\Lambda}$ to the above subcomplexes. This concludes the proof of Proposition 4.7. 
Remark 6.9 As mentioned in the introduction, the homotopy and localization argument used in the proof of Proposition 4.7 is inspired by an idea of H Hofer's. One could try to apply this idea directly to the proof that the pair-of-pants homomorphism $\Upsilon^{\Lambda}$ corresponds to the Morse theoretical interpretation of the loop product (see Proposition 2.6), without passing from the factorizations through the figure- 8 Floer and Morse complexes. This would involve showing that a Floer problem for $u$ : $\Sigma^{+} \rightarrow T^{*} M^{2}$ satisfying the boundary conditions

$$
(u(s), \mathscr{C} u(s+i)) \in N^{*}\left(\Delta_{M} \times \Delta_{M}\right) \text { for } s \geq 0,
$$

is homotopic to a Floer problem for $u$ satisfying

$$
\begin{array}{ll}
(u(s), \mathscr{C} u(s+i)) \in N^{*} \Delta_{M \times M} & \text { for } 0 \leq s \leq \alpha, \\
(u(s), \mathscr{C} u(s+i)) \in N^{*}\left(\Delta_{M} \times \Delta_{M}\right) & \text { for } s \geq \alpha .
\end{array}
$$

Here the trick of doubling the dimensions by using the algebraic Lemma 4.6 would not be needed, because the manifolds $\Delta_{M \times M}$ and $\Delta_{M} \times \Delta_{M}$ have the same dimension. However, a local isotopy between such manifolds cannot be partially orthogonal to $\Delta_{M} \times \Delta_{M}$, so the elliptic estimates of Section 5 would not be available and one would face compactness problems.

\section{Appendix: Morse constructions}

The aim of the first section of this appendix is to recall the construction of the Morse complex for functions defined on an infinite-dimensional Hilbert manifold. See Abbondandolo and Majer [1] for detailed proofs.

Many operations in singular homology can be read on the Morse complex (see for instance Schwarz [45], Fukaya [27; 28], Betz and Cohen [7], Viterbo [51]). Here we are interested only in functoriality, in the exterior homology product, and in the Umkehr map. The corresponding constructions - still in our infinite dimensional setting - are outlined in the subsequent sections.

\section{A.1 The Morse complex}

Let $\mathcal{M}$ be a (possibly infinite-dimensional) Hilbert manifold, that is, a Hausdorff paracompact topological space which is locally homeomorphic to a real Hilbert space and admits an atlas whose transition maps are smooth. If $X$ is a smooth vector field on $\mathcal{M}$ and $x \in \mathcal{M}$ is a singular point for $X$, ie $X(x)=0$, the Jacobian of $X$ at $x$ is a well-defined bounded operator on $T_{x} \mathcal{M}$, that we denote by $\nabla X(x)$. The singular point $x$ is said to be hyperbolic if the spectrum of $\nabla X(x)$ is disjoint from $i \mathbb{R}$, and 
the Morse index $i(x)$ of $x$ is the (possibly infinite) dimension of the $\nabla X(x)$-invariant closed linear subspace of $T_{x} \mathcal{M}$ associated to the part of the spectrum with positive real part. Hyperbolic singular points are isolated within the set of singular points. The vector field $X$ is said to be Morse if all its singular points are hyperbolic.

A real function $f \in C^{1}(\mathcal{M})$ is said to be a Lyapunov function for $X$ if $D f(p)[X(p)]<0$ for every $p \in \mathcal{M}$ which is not a singular point of $X$. If $X$ is Morse, then the set of singular points of $X$ coincides with the set of critical points of $f$, that we denote by $\operatorname{crit}(f)$ (in general, $\operatorname{crit}(f)$ is contained in the set of singular points of $X$ ). If $(t, p) \mapsto \phi(t, p)$ denotes the flow of the vector field $X$, the stable and unstable manifolds of the hyperbolic singular point $x$ are the subsets

$$
\begin{aligned}
& W^{s}(x ; X):=\{p \in \mathcal{M} \mid \phi(t, p) \rightarrow x \text { for } t \rightarrow+\infty\}, \\
& W^{u}(x ; X):=\{p \in \mathcal{M} \mid \phi(t, p) \rightarrow x \text { for } t \rightarrow-\infty\} .
\end{aligned}
$$

We just write $W^{s}(x)$ and $W^{u}(x)$ when the vector field $X$ is clear from the context. They are always injectively immersed smooth submanifolds of $\mathcal{M}$, and if $X$ admits a Lyapunov function $f$ and $i(x)<+\infty$ they are actually embedded submanifolds (if $i(x)=+\infty$, the same is true under a nondegeneracy condition on $f$; see Theorem 1.20 in [1]). Their tangent spaces at $x$ are the $\nabla X(x)$-invariant subspaces determined by the negative part (for the stable manifold) and the positive part (for the unstable manifold) of the spectrum of $\nabla X(x)$. In particular, $\operatorname{dim} W^{u}(x)=i(x)$. The Morse vector field $X$ is said to satisfy the Morse-Smale condition if for every pair of singular points $x, y$, the unstable manifold of $x$ is transverse to the stable manifold of $y$.

A Palais-Smale sequence for the pair $(X, f)$ is a sequence $\left(p_{h}\right) \subset \mathcal{M}$ such that $f\left(p_{h}\right)$ is bounded and $D f\left(p_{h}\right)\left[X\left(p_{h}\right)\right]$ is infinitesimal. The pair $(X, f)$ is said to satisfy the Palais-Smale condition if every Palais-Smale sequence has a converging subsequence.

Let $\mathcal{F}(\mathcal{M})$ be the set of all $C^{1}$ real functions $f$ on $\mathcal{M}$ which are bounded from below and for which there exists a smooth vector field $X$ on $\mathcal{M}$ such that:

(X1) $f$ is a Lyapunov function for $X$.

(X2) $X$ is Morse and all its singular points (ie the critical points of $f$ ) have finite Morse index.

(X3) The pair $(X, f)$ satisfies the Palais-Smale condition.

(X4) $X$ is forward complete (ie the flow $\phi(t, p)$ is defined for every $t \geq 0$ ).

Such a vector field is called a (negative) pseudo-gradient for $f$. The Morse index $i(x)$ of a critical point $x$ of $f$ does not depend on the choice of the pseudo-gradient $X$, and if $f$ has a nondegenerate Gateaux second differential $d^{2} f(x)$ at the critical point $x$, 
$i(x)$ coincides with the standard Morse index $i(x ; f)$, that is the dimension of a maximal linear subspace of $T_{x} \mathcal{M}$ on which $d^{2} f(x)$ is negative definite. The set of critical points of $f$ of Morse index $k$ is denoted by $\operatorname{crit}_{k}(f)$.

If $f$ is a smooth Morse function, bounded from below, and it satisfies the Palais-Smale condition with respect to some complete Riemannian metric $g$ on $\mathcal{M}$ (in the usual sense; see eg Chang [8]), then $-\operatorname{grad}_{g} f$ satisfies (X1)-(X4), so $f \in \mathcal{F}(\mathcal{M})$. However, it is useful to have a theory which allows also for nonregular functions $f$, as the example of the Lagrangian action functional introduced in Section 2.2 shows.

If $f \in \mathcal{F}(\mathcal{M})$, it is possible to perturb the smooth vector field $X$ generically, in such a way that (X1)-(X4) still hold, and:

(X5) $X$ satisfies the Morse-Smale condition.

In this paper, genericity for a pseudo-gradient $X$ is meant in a suitable complete metric space of vector fields which coincide with the original one up to order one at critical points, endowed with an adapted Whitney metric such that all the vector fields whose distance from the original one is less than 1 still satisfy (X1)-(X4). We shall not specify such metric space any further (see Abbondandolo and Majer [1] for more details).

Let us fix a real number $a$. Since $f$ is bounded from below, (X2) and (X3) imply that $f$ has finitely many critical points in the sublevel $\{f<a\}$. Using also (X4) and (X5), one can find open neighborhoods $\mathcal{U}(x)$ of each critical point $x$ in $\{f<a\}$ such that, if we set

$$
\mathcal{M}_{k}^{a}:=\bigcup_{\substack{x \in \operatorname{crit}(f) \\ f(x)<a \\ i(x) \leq k}} \phi([0,+\infty[\times \mathcal{U}(x)), \quad \forall k \in \mathbb{N} \cup\{\infty\},
$$

the (eventually constant) sequence $\left\{\mathcal{M}_{k}^{a}\right\}_{k \in \mathbb{N}}$ is a cellular filtration of $\mathcal{M}_{\infty}^{a}$. More precisely, if $M_{k}^{a}(f)$ denotes the free abelian group generated by the critical points in $\{f<a\}$ of Morse index $k$, one has

$$
H_{j}\left(\mathcal{M}_{k}^{a}, \mathcal{M}_{k-1}^{a}\right) \cong \begin{cases}M_{k}^{a}(f) & \text { if } j=k \\ 0 & \text { if } j \neq k\end{cases}
$$

and this isomorphism is uniquely determined by the choice of an orientation of each unstable manifold $W^{u}(x)$ of $x \in \operatorname{crit}_{k}(f) \cap\{f<a\}$. Moreover, $\mathcal{M}_{\infty}^{a}$ is a deformation retract of the sublevel $\{f<a\}$. Then the cellular complex of the cellular filtration $\left\{\mathcal{M}_{k}^{a}\right\}_{k \in \mathbb{N}}$ induces the structure of a chain complex on the graded group $M_{*}^{a}(f)$, whose homology is isomorphic to the singular homology of $\mathcal{M}_{\infty}^{a}$, hence to the singular homology of $\{f<a\}$. This chain complex is called the Morse complex of $(X, f)$ on 
the sublevel $\{f<a\}$. If we choose different neighborhoods $\mathcal{U}(x)$, we obtain the same chain complex. Actually, the boundary homomorphism

$$
\partial_{k}: M_{k}^{a}(f) \rightarrow M_{k-1}^{a}(f)
$$

can be expressed in terms of the standard generators of $M_{k}^{a}(f)$, that is the critical points $x$ such that $f(x)<a$ and $i(x)=k$, as

$$
\partial_{k} x=\sum_{\substack{y \in \operatorname{crit}(f) \\ i(y)=k-1}} n_{\partial}(x, y) y,
$$

where $n_{\partial}$ is defined as follows. The chosen orientation of each unstable manifold $W^{u}(x)$ induces a co-orientation of each stable manifold. By (X5), each intersection $W^{u}(x) \cap W^{s}(y)$ is a transverse intersection of an oriented submanifold of dimension $i(x)$ and a co-oriented submanifold of codimension $i(y)$. So $W^{u}(x) \cap W^{s}(y)$ is an oriented submanifold of dimension $i(x)-i(y)$. When $i(x)-i(y)=1, W^{u}(x) \cap W^{s}(y)$ consists of finitely many flow orbits, and $n_{\partial}(x, y)$ is the number of those orbits on which the direction of $X$ agrees with the orientation, minus the number of the other ones.

The Morse complex of $(X, f)$ on $\mathcal{M}$ is a chain complex on the graded free abelian group $M_{*}(f)$ generated by all the critical points of $f$, and it is defined either by taking a direct limit of $\left\{M_{*}^{a}(f), \partial_{*}\right\}$ for $a \uparrow \infty$, or - equivalently - by formula (A-1), which does not depend on $a$. In order to use the vector field $X$ to construct a cellular filtration of the whole $\mathcal{M}$, one would need that every critical point $x$ does not belong to the closure of the union of all the unstable manifolds of critical points $y$ of Morse index $i(y) \leq i(x)$. The latter fact is implied by the Morse-Smale condition when there is a finite number of critical points of any given index, but it mail fail in the general case. This is why we work with sublevels and we define the Morse complex on $\mathcal{M}$ as a direct limit. Since the homology functor commutes with direct limits, the first definition implies that the homology of $\left\{M_{*}(f), \partial_{*}\right\}$ is isomorphic to the singular homology of $\mathcal{M}$.

If we change the orientations of the unstable manifolds, we get isomorphic Morse complexes. The same is true if we choose a different pseudo-gradient vector field $X$ satisfying (X1)-(X5) with respect to the same $f$. Therefore, the Morse complex of $f$ is well-defined up to isomorphism, for every $f \in \mathcal{F}(\mathcal{M})$.

It is also useful to consider the following relative version of the Morse complex. Let $\mathcal{A}$ be an open subset of the Hilbert manifold $\mathcal{M}$. Let $\mathcal{F}(\mathcal{M}, \mathcal{A})$ be the set of all $C^{1}$ real functions $f$ on $\mathcal{M}$ which are bounded from below on $\mathcal{M} \backslash \mathcal{A}$ and for which there is a smooth vector field $X$ on $\mathcal{M}$ such that: 
$\left(\mathrm{X} 1^{\prime}\right) \quad f$ is a Lyapunov function for $X$ on $\mathcal{M} \backslash \overline{\mathcal{A}}$.

$\left(\mathrm{X} 2^{\prime}\right) \quad X$ is Morse on $\mathcal{M} \backslash \overline{\mathcal{A}}$ and all its singular points in this open set have finite Morse index.

$\left(\mathrm{X}^{\prime}\right)$ The pair $(X, f)$ satisfies the Palais-Smale condition on $\mathcal{M} \backslash \overline{\mathcal{A}}$.

$\left(\mathrm{X} 4^{\prime}\right) \mathcal{A}$ is positively invariant with respect to the flow of $X$ (ie $p \in \mathcal{A}$ implies $\phi(t, p) \in \mathcal{A}$ for every $t \geq 0$ for which the flow is defined), and $X$ is forward complete relative to $\mathcal{A}$ (ie if $\phi(t, p)$ is not defined for all $t \geq 0$, then $\phi(t, p) \in \mathcal{A}$ for $t$ large enough).

In particular, $\left(\mathrm{X}^{\prime}\right)$ implies that $X$ has no singular points on the boundary of $\mathcal{A}$, hence $f$ has no critical points on the boundary of $\mathcal{A}$. In fact, a sequence $\left(p_{h}\right) \subset \mathcal{M} \backslash \overline{\mathcal{A}}$ which converges to a singular point of $X$ on the boundary of $\mathcal{A}$ would be a Palais-Smale sequence which does not converge in $\mathcal{M} \backslash \overline{\mathcal{A}}$. By a generic perturbation, we may assume that $X$ satisfies also:

$\left(\mathrm{X}^{\prime}\right) \quad X$ satisfies the Morse-Smale condition on $\mathcal{M} \backslash \overline{\mathcal{A}}$.

Then the relative Morse complex of $(X, f)$ on $(\mathcal{M}, \mathcal{A})$ is constructed as before, but taking into account only the critical points of $f$ in $\mathcal{M} \backslash \overline{\mathcal{A}}$. The homology of this chain complex is isomorphic to the relative singular homology $H_{*}(\mathcal{M}, \mathcal{A})$. As before, the isomorphism class of the Morse complex does not depend on the pseudo-gradient $X$, but only on the function $f$.

\section{A.2 Functoriality}

Let $\varphi: \mathcal{M}_{1} \rightarrow \mathcal{M}_{2}$ be a smooth map between Hilbert manifolds. Let $f_{1} \in \mathcal{F}\left(\mathcal{M}_{1}\right)$ and $f_{2} \in \mathcal{F}\left(\mathcal{M}_{2}\right)$, and let $X_{1}$ and $X_{2}$ be corresponding Morse-Smale pseudo-gradients, ie smooth vector fields satisfying (X1)-(X5). We denote by $\phi^{1}$ and $\phi^{2}$ the corresponding flows. We assume that:

Each $y \in \operatorname{crit}\left(f_{2}\right)$ is a regular value of $\varphi$.

$$
x \in \operatorname{crit}\left(f_{1}\right), \varphi(x) \in \operatorname{crit}\left(f_{2}\right) \quad \Longrightarrow \quad i\left(x ; f_{1}\right) \geq i\left(\varphi(x) ; f_{2}\right) .
$$

The set of critical points of $f_{2}$ is discrete, and in many cases (for instance, if $\varphi$ is a Fredholm map) the set of regular values of $\varphi$ is generic (ie, it is a countable intersection of open and dense sets), by Sard-Smale theorem [48]. In such a situation, condition (A-2) can be achieved by arbitrary small (in several senses) perturbations of $\varphi$ or of $f_{2}$. Also condition (A-3) can be achieved by an arbitrary small perturbation of $\varphi$ or of $f_{2}$, simply by requiring that the image of the set of critical points of $f_{1}$ by $\varphi$ does not meet the set of critical point of $f_{2}$. 
By (A-2) and (A-3), up to the perturbation of the vector field $X_{1}$ and $X_{2}$, we may assume that

(A-4) $\quad \forall x \in \operatorname{crit}\left(f_{1}\right), \forall y \in \operatorname{crit}\left(f_{2}\right),\left.\quad \varphi\right|_{W^{u}\left(x ; X_{1}\right)}$ is transverse to $W^{s}\left(y ; X_{2}\right)$.

Indeed, by (A-2) and (A-3) one can perturb $X_{1}$ in such a way that if $p \in W^{u}\left(x ; X_{1}\right)$ and $\varphi(p)$ is a critical point of $f_{2}$ then rank $\left.D \varphi(p)\right|_{T_{p} W^{u}(x)} \geq i\left(\varphi(p) ; f_{2}\right)$. The possibility of perturbing $X_{2}$ so that (A-4) holds is now a consequence of the following fact: if $W$ is a finite dimensional manifold and $\psi: W \rightarrow \mathcal{M}_{2}$ is a smooth map such that for every $p \in W$ with $\psi(p) \in \operatorname{crit}\left(f_{2}\right)$ there holds rank $D \psi(p) \geq i\left(\varphi(p) ; f_{2}\right)$, then the set of pseudo-gradient vector fields $X_{2}$ for $f_{2}$ on $\mathcal{M}_{2}$ such that the map $\psi$ is transverse to the stable manifold of every critical point of $f_{2}$ is generic.

The transversality condition (A-4) ensures that if $x \in \operatorname{crit}\left(f_{1}\right)$ and $y \in \operatorname{crit}\left(f_{2}\right)$, then

$$
W(x, y):=W^{u}\left(x ; X_{1}\right) \cap \varphi^{-1}\left(W^{s}\left(y ; X_{2}\right)\right)
$$

is a submanifold of dimension $i\left(x ; f_{1}\right)-i\left(y ; f_{2}\right)$. If $W^{u}\left(x ; X_{1}\right)$ is oriented and the normal bundle of $W^{s}\left(y ; X_{2}\right)$ in $\mathcal{M}_{2}$ is oriented, the manifold $W(x, y)$ carries a canonical orientation. In particular, if $i\left(x ; f_{1}\right)=i\left(y ; f_{2}\right), W(x, y)$ is a discrete set, each of whose point carries an orientation sign \pm 1 . The transversality condition (A-4) and the fact that $W^{u}\left(x ; X_{1}\right)$ has compact closure in $\mathcal{M}_{1}$ imply that the discrete set $W(x, y)$ is also compact, so it is a finite set and we denote by $n_{\varphi}(x, y) \in \mathbb{Z}$ the algebraic sum of the corresponding orientation signs. We can then define the homomorphism

$$
M_{k} \varphi: M_{k}\left(f_{1}\right) \rightarrow M_{k}\left(f_{2}\right), \quad\left(M_{k} \varphi\right) x=\sum_{y \in \text { crit }_{k}\left(f_{2}\right)} n_{\varphi}(x, y) y,
$$

for every $x \in \operatorname{crit}_{k}\left(f_{1}\right)$.

We claim that $M_{*} \varphi$ is a chain map from the Morse complex of $\left(f_{1}, X_{1}\right)$ to the Morse complex of $\left(f_{2}, X_{2}\right)$, and that the corresponding homomorphism in homology coincides - via the isomorphism described in Appendix A.1 - with the homomorphism $\varphi_{*}: H_{*}\left(\mathcal{M}_{1}\right) \rightarrow H_{*}\left(\mathcal{M}_{2}\right)$.

Indeed, let us fix a real number $a_{1}$, and let $a_{2}$ be larger than the maximum of $f_{2}$ on the image by $\varphi$ of the union of all unstable manifolds of critical points of $f_{1}$ in $\left\{f<a_{1}\right\}$ (the latter is a compact set). Then we can find open neighborhoods $\mathcal{U}_{i}(x), i=1,2$, of each critical point $x \in \operatorname{crit}\left(f_{i}\right)$, such that the sequence of open sets

$$
\mathcal{M}_{i, k}^{a_{i}}=\bigcup_{\substack{x \in \operatorname{crit}\left(f_{i}\right) \\ f_{i}(x)<a_{i} \\ i\left(x ; f_{i}\right) \leq k}} \phi^{i}\left(\left[0,+\infty\left[\times \mathcal{U}_{i}(x)\right), \quad k \in \mathbb{N} \cup\{\infty\}, i=1,2,\right.\right.
$$


is a cellular filtration of $\mathcal{M}_{i, \infty}^{a_{i}}$, which is a deformation retract of the sublevel

$$
\left\{p \in \mathcal{M}_{i} \mid f_{i}(p)<a_{i}\right\},
$$

and

$$
\varphi\left(\mathcal{M}_{1, \infty}^{a_{1}}\right) \subset\left\{p \in \mathcal{M}_{2} \mid f_{2}(p)<a_{2}\right\} .
$$

By the transversality assumption (A-4), if $p$ belongs to $W^{u}\left(x ; X_{1}\right)$ with $f_{1}(x)<a_{1}$, then $\varphi(p)$ belongs to the stable manifold of some critical point $y \in \operatorname{crit}\left(f_{2}\right)$ with $i\left(y ; f_{2}\right) \leq i\left(x ; f_{1}\right)$ and $f_{2}(y)<a_{2}$. A standard compactness-transversality argument shows that, up to the replacement of the neighborhoods $\mathcal{U}_{1}(x), x \in \operatorname{crit}\left(f_{1}\right)$, by smaller ones, we may assume that

$$
\begin{array}{r}
p \in \mathcal{M}_{1, k}^{a_{1}} \Longrightarrow \varphi(p) \in W^{s}\left(y ; X_{2}\right) \text { for some } y \in \operatorname{crit}\left(f_{2}\right) \text { with } i\left(y ; f_{2}\right) \leq k \\
\text { and } f_{2}(y)<a_{2}
\end{array}
$$

Since the set $\mathcal{M}_{2, k}^{a_{2}}$ is a $\phi^{2}$-positively invariant open neighborhood of the set of the critical points of $f_{2}$ in $\left\{f_{2}<a_{2}\right\}$ whose Morse index does not exceed $k$, it is easy to find a continuous function $t_{0}: \mathcal{M}_{1, \infty}^{a_{1}} \rightarrow[0,+\infty[$ such that

$$
p \in \mathcal{M}_{1, k}^{a_{1}} \quad \Longrightarrow \quad \phi^{2}\left(t_{0}(p), \varphi(p)\right) \in \mathcal{M}_{2, k}^{a_{2}}, \quad \forall k \in \mathbb{N} .
$$

Thus, $\psi(p):=\phi^{2}\left(t_{0}(p), \varphi(p)\right)$ is a cellular map from $\left\{\mathcal{M}_{1, k}^{a_{1}}\right\}_{k \in \mathbb{N}}$ to $\left\{\mathcal{M}_{2, k}^{a_{2}}\right\}_{k \in \mathbb{N}}$, and it is easy to check that the induced cellular homomorphism

$$
\psi_{*}: H_{*}\left(\mathcal{M}_{1, *}^{a_{1}}, \mathcal{M}_{1, *-1}^{a_{1}}\right) \rightarrow H_{*}\left(\mathcal{M}_{2, *}^{a_{2}}, \mathcal{M}_{2, *-1}^{a_{2}}\right)
$$

coincides with the restriction $M_{*} \varphi: M_{*}^{a_{1}}\left(f_{1}\right) \rightarrow M_{*}^{a_{2}}\left(f_{2}\right)$, once we identify the group $H_{k}\left(\mathcal{M}_{i, k}^{a_{i}}, \mathcal{M}_{i, k-1}^{a_{i}}\right)$ with $M_{k}^{a_{i}}\left(f_{i}\right)$, by taking the orientations of the unstable manifolds into account. Then, everything follows from the naturality of cellular homology, from the fact that the inclusions $j_{i}: \mathcal{M}_{i, \infty}^{a_{i}} \hookrightarrow\left\{p \in \mathcal{M}_{i} \mid f\left(p_{i}\right)<a_{i}\right\}$ are homotopy equivalences, from the fact that $j_{2} \circ \psi$ is homotopic to $\varphi \circ j_{1}$, and by taking a direct limit for $a_{1} \uparrow+\infty$.

The above construction has an obvious extension to the case of a smooth map $\varphi$ between two pairs $\left(\mathcal{M}_{1}, \mathcal{A}_{1}\right)$ and $\left(\mathcal{M}_{2}, \mathcal{A}_{2}\right)$, where $\mathcal{A}_{i}$ is an open subset of $\mathcal{M}_{i}$, for $i=1,2$.

Remark A.1 We recall that if two chain maps between free chain complexes induce the same homomorphism in homology, they are chain homotopic. So from the functoriality of singular homology, we deduce that $M_{*} \varphi \circ M_{*} \psi$ and $M_{*} \varphi \circ \psi$ are chain homotopic. Actually, a chain homotopy between these two chain maps could be constructed in a direct way. 
Remark A.2 Consider the following particular but important case: $\mathcal{M}_{1}=\mathcal{M}_{2}$ and $\varphi=\mathrm{id}$. Then (A-2) holds automatically, and (A-3) means asking that every common critical point $x$ of $f_{1}$ and $f_{2}$ must satisfy $i\left(x ; f_{1}\right) \geq i\left(x ; f_{2}\right)$. In this case, the above construction produces a chain map from $M_{*}\left(f_{1}\right)$ to $M_{*}\left(f_{2}\right)$ which induces the identity map in homology (after the identification with singular homology).

Remark A.3 For future reference, let us stress the fact that if it is already known that $p \in W^{u}\left(x ; X_{1}\right)$ and $\varphi(p) \in \operatorname{crit}\left(f_{2}\right)$ imply $\left.\operatorname{rank} D \varphi(p)\right|_{T_{p} W^{u}\left(x ; X_{1}\right)} \geq i\left(\varphi(p) ; f_{2}\right)$, then condition (A-2) is redundant, condition (A-3) holds automatically, and there is no need of perturbing the vector field $X_{1}$ on $\mathcal{M}_{1}$.

\section{A.3 The exterior homology product}

Let $\mathcal{M}_{1}, \mathcal{M}_{2}$ be Hilbert manifolds, let $f_{1} \in \mathcal{F}\left(\mathcal{M}_{1}\right), f_{2} \in \mathcal{F}\left(\mathcal{M}_{2}\right)$, and let $X_{1}$ and $X_{2}$ be corresponding Morse-Smale pseudo-gradients. If we denote by $f_{1} \oplus f_{2}$ the function on $\mathcal{M}_{1} \times \mathcal{M}_{2}$,

$$
f_{1} \oplus f_{2}\left(p_{1}, p_{2}\right):=f_{1}\left(p_{1}\right)+f_{2}\left(p_{2}\right),
$$

we see that $f_{1} \oplus f_{2}$ belongs to $\mathcal{F}\left(\mathcal{M}_{1} \times \mathcal{M}_{2}\right)$, and

$$
X_{1} \oplus X_{2}\left(p_{1}, p_{2}\right):=\left(X_{1}\left(p_{1}\right), X_{2}\left(p_{2}\right)\right),
$$

satisfies $(\mathrm{X} 1)-(\mathrm{X} 5)$ with respect to $f_{1} \oplus f_{2}$. Moreover,

hence

$$
\operatorname{crit}_{\ell}\left(f_{1} \oplus f_{2}\right)=\bigcup_{j+k=\ell} \operatorname{crit}_{j}\left(f_{1}\right) \times \operatorname{crit}_{k}\left(f_{2}\right),
$$

$$
M_{\ell}\left(f_{1} \oplus f_{2}\right)=\bigoplus_{j+k=\ell} M_{j}\left(f_{1}\right) \otimes M_{k}\left(f_{2}\right) .
$$

If we fix orientations for the unstable manifold of each critical point of $f_{1}, f_{2}$, and we endow the unstable manifold of each $\left(x_{1}, x_{2}\right) \in \operatorname{crit}\left(f_{1} \oplus f_{2}\right)$,

$$
W^{u}\left(\left(x_{1}, x_{2}\right)\right)=W^{u}\left(x_{1}\right) \times W^{u}\left(x_{2}\right),
$$

with the product orientation, we see that the boundary homomorphism in the Morse complex of $\left(f_{1} \oplus f_{2}, X_{1} \oplus X_{2}\right)$ is given by

$$
\partial\left(x_{1}, x_{2}\right)=\left(\partial x_{1}, x_{2}\right)+(-1)^{i\left(x_{1}\right)}\left(x_{1}, \partial x_{2}\right), \quad \forall x_{i} \in \operatorname{crit}\left(f_{i}\right), i=1,2 .
$$

We conclude that the Morse complex of $\left(f_{1} \oplus f_{2}, X_{1} \oplus X_{2}\right)$ is the tensor product of the Morse complexes of $\left(f_{1}, X_{1}\right)$ and $\left(f_{2}, X_{2}\right)$. So, using the natural homomorphism 
from the tensor product of the homology of two chain complexes to the homology of the tensor product of the two complexes, we obtain the homomorphism

$$
H_{j} M\left(f_{1}\right) \otimes H_{k} M\left(f_{2}\right) \rightarrow H_{j+k} M\left(f_{1} \oplus f_{2}\right) .
$$

We claim that this homomorphism corresponds to the exterior product homomorphism

$$
H_{j}\left(\mathcal{M}_{1}\right) \otimes H_{k}\left(\mathcal{M}_{2}\right) \stackrel{\times}{\longrightarrow} H_{j+k}\left(\mathcal{M}_{1} \times \mathcal{M}_{2}\right),
$$

via the isomorphism between Morse homology and singular homology described in Appendix A.1.

Indeed, the cellular filtration in $\left\{f_{1}<a_{1}\right\} \times\left\{f_{2}<a_{2}\right\}$ can be chosen to be generated by small product neighborhoods of the critical points,

$$
\begin{aligned}
\mathcal{W}_{\ell}^{a_{1}, a_{2}}= & \bigcup_{\substack{\left(x_{1}, x_{2}\right) \in \operatorname{crit}\left(f_{1} \oplus f_{2}\right) \\
f_{1}\left(x_{1}\right)<a_{1}, f_{2}\left(x_{2}\right)<a_{2} \\
i\left(x_{1}\right)+i\left(x_{2}\right)=\ell}} \phi^{1}\left(\left[0,+\infty\left[\times \mathcal{U}_{1}\left(x_{1}\right)\right) \times \phi^{2}\left(\left[0,+\infty\left[\times \mathcal{U}_{2}\left(x_{2}\right)\right)\right.\right.\right.\right. \\
= & \bigcup_{j+k=\ell} \mathcal{M}_{1, j}^{a_{1}} \times \mathcal{M}_{2, k}^{a_{2}} .
\end{aligned}
$$

By excision and by the Künneth theorem, together with the fact that we are dealing with free abelian groups, one easily obtains that

$$
H_{\ell}\left(\mathcal{W}_{\ell}^{a_{1}, a_{2}}, \mathcal{W}_{\ell-1}^{a_{1}, a_{2}}\right) \cong \bigoplus_{j+k=\ell} H_{j}\left(\mathcal{M}_{1, j}^{a_{1}}, \mathcal{M}_{1, j-1}^{a_{1}}\right) \otimes H_{k}\left(\mathcal{M}_{2, k}^{a_{2}}, \mathcal{M}_{2, k-1}^{a_{2}}\right),
$$

and that the boundary homomorphism of the cellular filtration $\mathcal{W}_{*}^{a_{1}, a_{2}}$ is the tensor product of the boundary homomorphisms of the cellular filtrations $\mathcal{M}_{1, *}^{a_{1}}$ and $\mathcal{M}_{2, *}^{a_{2}}$. Passing to homology, we find that (A-5) corresponds to the exterior homology product

$$
H_{j}\left(\mathcal{M}_{1, \infty}^{a_{1}}\right) \otimes H_{k}\left(\mathcal{M}_{2, \infty}^{a_{2}}\right) \stackrel{\times}{\longrightarrow} H_{j+k}\left(\mathcal{M}_{1, \infty}^{a_{1}} \times \mathcal{M}_{2, \infty}^{a_{2}}\right)=H_{j+k}\left(\mathcal{W}_{\infty}^{a_{1}, a_{2}}\right),
$$

by the usual identification of the cellular complex to the Morse complex induced by a choice of orientations for the unstable manifolds. But using the fact that the inclusion $\mathcal{M}_{1, \infty}^{a_{1}} \hookrightarrow\left\{f_{1}<a_{1}\right\}$ and $\mathcal{M}_{2, \infty}^{a_{2}} \hookrightarrow\left\{f_{2}<a_{2}\right\}$ are homotopy equivalences, and by considering a direct limit for $a_{1}, a_{2} \uparrow+\infty$, we conclude that (A-5) corresponds to $(\mathrm{A}-6)$.

\section{A.4 Intersection products}

Let $\mathcal{M}_{0}$ be a Hilbert manifold, and let $\pi: \mathcal{E} \rightarrow \mathcal{M}_{0}$ be a smooth rank- $n$ oriented real vector bundle over $\mathcal{M}_{0}$. It is easy to describe the Thom isomorphism

$$
\tau: H_{k}\left(\mathcal{E}, \mathcal{E} \backslash \mathcal{M}_{0}\right) \stackrel{\cong}{\longrightarrow} H_{k-n}\left(\mathcal{M}_{0}\right), \quad \alpha \mapsto \tau_{\mathcal{E}} \cap \alpha,
$$


in a Morse theoretical way $\left(\tau_{\mathcal{E}} \in H^{n}\left(\mathcal{E}, \mathcal{E} \backslash \mathcal{M}_{0}\right)\right.$ denotes the Thom class of the vector bundle $\mathcal{E}$ ).

Indeed, let $f_{0} \in \mathcal{F}\left(\mathcal{M}_{0}\right)$ and let $X_{0}$ be a Morse-Smale pseudo-gradient for $f_{0}$. The choice of a Riemannian structure on the vector bundle $\mathcal{E}$ determines the smooth function

$$
f_{1}(\xi):=f_{0}(\pi(\xi))-|\xi|^{2}, \quad \forall \xi \in \mathcal{E} .
$$

The choice of a connection on $\mathcal{E}$ induces the horizontal-vertical splitting and the isomorphism

$$
T_{\xi} \mathcal{E}=T_{\xi}^{h} \mathcal{E} \oplus T_{\xi}^{v} \mathcal{E} \cong T_{\pi(\xi)} \mathcal{M}_{0} \oplus \pi^{-1}(\pi(\xi)) .
$$

By the above identifications, we can define the tangent vector field $X_{1}$ on the total space $\mathcal{E}$ by

$$
X_{1}(\xi):=\left(X_{0}(\pi(\xi)), \xi\right)
$$

It is readily seen that $\left(f_{1}, X_{1}\right)$ satisfies conditions $\left(\mathrm{X} 1^{\prime}\right)-\left(\mathrm{X} 5^{\prime}\right)$ of Appendix A.1 on the pair $\left(\mathcal{E}, \mathcal{E} \backslash \overline{\mathcal{U}_{1}}\right), \mathcal{U}_{1}$ being the set of vectors $\xi$ in the total space $\mathcal{E}$ with $|\xi|<1$. Therefore, the homology of the relative Morse complex of $\left(f_{1}, X_{1}\right)$ on $\left(\mathcal{E}, \mathcal{E} \backslash \overline{\mathcal{U}_{1}}\right)$ is isomorphic to the singular homology of the pair $\left(\mathcal{E}, \mathcal{E} \backslash \overline{\mathcal{U}_{1}}\right)$, that is to the singular homology of $\left(\mathcal{E}, \mathcal{E} \backslash \mathcal{M}_{0}\right)$. Actually, the critical points of $f_{1}$ are contained in the zero-section of $\mathcal{E}$, and if we identify such a zero section with $\mathcal{M}_{0}$, we have

$\operatorname{crit}_{k}\left(f_{1}\right)=\operatorname{crit}_{k-n}\left(f_{0}\right), \quad T_{x} W^{u}\left(x ; X_{1}\right)=T_{x} W^{u}\left(x ; X_{0}\right) \oplus \pi^{-1}(x), \quad \forall x \in \operatorname{crit}\left(f_{0}\right)$,

so the orientation of the vector bundle $\mathcal{E}$ allows to associate an orientation of $W^{u}\left(x ; X_{1}\right)$ to each orientation of $W^{u}\left(x ; X_{0}\right)$. Then the relative Morse complex of $\left(f_{1}, X_{1}\right)$ on $\left(\mathcal{E}, \mathcal{E} \backslash \overline{\mathcal{U}_{1}}\right)$ is obtained from the Morse complex of $\left(f_{0}, X_{0}\right)$ on $\mathcal{M}_{0}$ by a $-n$-shift in the grading:

$$
M_{k}\left(f_{1}, X_{1}\right)=M_{k-n}\left(f_{0}, X_{0}\right),
$$

and one can show that the isomorphism $\tau$ - read on the Morse complexes by the isomorphisms described in Appendix A.1 - is induced by the identity mapping

$$
M_{k}\left(f_{1}, X_{1}\right) \stackrel{\text { id }}{\longrightarrow} M_{k-n}\left(f_{0}, X_{0}\right) .
$$

See Cohen and Schwarz [18] for more details.

Consider now the general case of a closed embedding $e: \mathcal{M}_{0} \hookrightarrow \mathcal{M}$, assumed to be of codimension $n$ and co-oriented. The above description of the Thom isomorphism associated to the normal bundle $N \mathcal{M}_{0}$ of $\mathcal{M}_{0}$ and the discussion about functoriality of Appendix A.2 provide us with a Morse theoretical description of the Umkehr map

$$
e_{!}: H_{k}(\mathcal{M}) \rightarrow H_{k-n}\left(\mathcal{M}_{0}\right) \text {. }
$$


It is actually useful to identify an open neighborhood of $\mathcal{M}_{0}$ with $N \mathcal{M}_{0}$ by the tubular neighborhood theorem, to consider again the open unit ball $\mathcal{U}_{1}$ around the zero section of $N \mathcal{M}_{0}$, and to see the Umkehr map as the composition

$$
H_{j}(\mathcal{M}) \stackrel{i_{*}}{\longrightarrow} H_{j}\left(\mathcal{M}, \mathcal{M} \backslash \overline{\mathcal{U}_{1}}\right) \cong H_{j}\left(N \mathcal{M}_{0}, N \mathcal{M}_{0} \backslash \mathcal{M}_{0}\right) \stackrel{\tau}{\longrightarrow} H_{j-n}\left(\mathcal{M}_{0}\right),
$$

the map $i: \mathcal{M} \hookrightarrow\left(\mathcal{M}, \mathcal{M} \backslash \overline{\mathcal{U}_{1}}\right)$ being the inclusion. Let $f_{0}, X_{0}, f_{1}, X_{1}$ be as above. We use the symbols $f_{1}$ and $X_{1}$ also to denote arbitrary extensions of $f_{1}$ and $X_{1}$ to the whole $\mathcal{M}$. Let $f \in \mathcal{F}(\mathcal{M})$, and let $X$ be a Morse-Smale pseudo-gradient for $f$. Since we would like to achieve transversality by perturbing $X$ and $X_{0}$, but keeping $X_{1}$ of product-type near $\mathcal{M}_{0}$, we need the condition

$$
x \in \operatorname{crit}(f) \cap \mathcal{M}_{0} \quad \Longrightarrow \quad i(x ; f) \geq n,
$$

which implies that up to perturbing $X$ we may assume that the unstable manifold of each critical point of $f$ is transversal to $\mathcal{M}_{0}$. Assumption (A-2) is automatically satisfied by the triplet $\left(i, f, f_{1}\right)$, while (A-3) is equivalent to asking that

$$
x \in \operatorname{crit}(f) \cap \operatorname{crit}\left(f_{0}\right) \quad \Longrightarrow \quad i(x ; f) \geq i\left(x ; f_{0}\right)+n .
$$

Conditions (A-7) and (A-8) are implied by the generic assumption $\operatorname{crit}(f) \cap \mathcal{M}_{0}=\varnothing$. By the arguments of Appendix A.2 applied to the map $i$ (in particular, condition (A-4)), we see that up to perturbing $X$ and $X_{0}$ (keeping $X_{1}$ of product-type near $\mathcal{M}_{0}$ ), we may assume that for every $x \in \operatorname{crit}(f), y \in \operatorname{crit}\left(f_{0}\right)$, the intersection

$$
W^{u}(x ; X) \cap W^{s}\left(y ; X_{1}\right)=W^{u}(x ; X) \cap W^{s}\left(y ; X_{0}\right)
$$

is transverse in $\mathcal{M}$, hence it is a submanifold of dimension $i(x ; f)-i\left(y ; f_{0}\right)-n$. If we fix an orientation of the unstable manifold of each critical point of $f$ and $f_{0}$, these intersections are canonically oriented. Compactness and transversality imply that when $i\left(y ; f_{0}\right)=i(x ; f)-n$, this intersection is a finite set of points, each of which comes with an orientation sign \pm 1 . Denoting by $n_{e_{!}}(x, y)$ the algebraic sum of these signs, we conclude that the homomorphism

$$
M_{k}(f) \rightarrow M_{k-n}\left(f_{0}\right), \quad x \mapsto \sum_{\substack{y \in \operatorname{crit}\left(f_{0}\right) \\ i\left(y ; f_{0}\right)=k-n}} n_{e !}(x, y) y, \quad \forall x \in \operatorname{crit}_{k}(f),
$$

is a chain map of degree $-n$, and that it induces the Umkehr map $e$ ! in homology.

Let us conclude this section by describing the Morse theoretical interpretation of the intersection product in homology. See Betz and Cohen [7] for the Morse theoretical description of more general cohomology operations. Let $M$ be a finite-dimensional 
oriented manifold, and consider the diagonal embedding $e: \Delta_{M} \hookrightarrow M \times M$, which is $n-$ codimensional and co-oriented. The intersection product is defined by the composition

$$
H_{j}(M) \otimes H_{k}(M) \stackrel{\times}{\longrightarrow} H_{j+k}(M \times M) \stackrel{e_{!}}{\longrightarrow} H_{j+k-n}\left(\Delta_{M}\right) \cong H_{j+k-n}(M),
$$

and it is denoted by

$$
\text { •: } H_{j}(M) \otimes H_{k}(M) \longrightarrow H_{j+k-n}(M) \text {. }
$$

The above description of $e_{\text {! }}$ and the description of the exterior homology product $x$ given in Appendix A.3 immediately yield the following description of $\bullet$. Let $f_{i} \in$ $\mathcal{F}(M), i=1,2,3$, and let $X_{i}$ be corresponding Morse-Smale pseudo-gradients (here we could assume that the $f_{i}$ 's are smooth Morse functions, and that $X_{i}=-\operatorname{grad}_{g} f_{i}$ with respect to three suitable complete metrics $g_{i}$ on $M$ ). The nondegeneracy conditions (A-7) and (A-8), which are used to represent $e_{!}$, are now

$$
x \in \operatorname{crit}\left(f_{1}\right) \cap \operatorname{crit}\left(f_{2}\right) \Longrightarrow i\left(x ; f_{1}\right)+i\left(x ; f_{2}\right) \geq n,
$$

$(\mathrm{A}-10) \quad x \in \operatorname{crit}\left(f_{1}\right) \cap \operatorname{crit}\left(f_{2}\right) \cap \operatorname{crit}\left(f_{3}\right) \Longrightarrow i\left(x ; f_{1}\right)+i\left(x ; f_{2}\right) \geq i\left(x ; f_{3}\right)+n$.

These conditions are implied for instance by the generic assumption that $f_{1}$ and $f_{2}$ do not have common critical points. We can now perturb the vector fields $X_{1}, X_{2}$ and $X_{3}$ in such a way that for every triplet $x_{i} \in \operatorname{crit}\left(f_{i}\right), i=1,2,3$, the intersection

$$
W^{u}\left(\left(x_{1}, x_{2}\right) ; X_{1} \oplus X_{2}\right) \cap a\left(W^{s}\left(x_{3} ; X_{3}\right)\right),
$$

where $a: M \rightarrow M \times M$ is the map $a(p)=(p, p)$, is transverse in $M \times M$, hence it is an oriented submanifold of $\Delta_{M}$ of dimension $i\left(x_{1} ; f_{1}\right)+i\left(x_{2} ; f_{2}\right)-i\left(x_{3} ; f_{3}\right)-n$. By compactness and transversality, when $i\left(x_{3} ; f_{3}\right)=i\left(x_{1} ; f_{1}\right)+i\left(x_{2} ; f_{2}\right)-n$, this intersection, which can also be written as

$$
\left\{(p, p) \in W^{u}\left(x_{1} ; X_{1}\right) \times W^{u}\left(x_{2} ; X_{2}\right) \mid p \in W^{s}\left(x_{3} ; X_{3}\right)\right\},
$$

is a finite set of points, each of which comes with an orientation sign \pm 1 . Denoting by $n_{\bullet}\left(x_{1}, x_{2} ; x_{3}\right)$ the algebraic sum of these signs, we conclude that the homomorphism

$$
\begin{aligned}
M_{j}\left(f_{1}, g_{1}\right) \otimes M_{k}\left(f_{2}, g_{2}\right) & \rightarrow M_{j+k-n}\left(f_{3}, g_{3}\right), \\
x_{1} \otimes x_{2} & \mapsto \sum_{\substack{x_{3} \in \operatorname{crit}\left(f_{3}\right) \\
i\left(x_{3} ; f_{3}\right)=j+k-n}} n_{\bullet}\left(x_{1}, x_{2} ; x_{3}\right) x_{3},
\end{aligned}
$$

where $x_{1} \in \operatorname{crit}_{j}\left(f_{1}\right), x_{2} \in \operatorname{crit}_{k}\left(f_{2}\right)$, is a chain map of degree $-n$ from $M\left(f_{1}\right) \otimes M\left(f_{2}\right)$ to $M\left(f_{3}\right)$, and that it induces the intersection product $\bullet$ in homology. 


\section{References}

[1] A Abbondandolo, P Majer, Lectures on the Morse complex for infinite-dimensional manifolds, from: "Morse theoretic methods in nonlinear analysis and in symplectic topology", (P Biran, O Cornea, F Lalonde, editors), NATO Sci. Ser. II Math. Phys. Chem. 217, Springer, Dordrecht (2006) 1-74 MR2276948

[2] A Abbondandolo, A Portaluri, M Schwarz, The homology of path spaces and Floer homology with conormal boundary conditions, J. Fixed Point Theory Appl. 4 (2008) 263-293 MR2465553

[3] A Abbondandolo, M Schwarz, Notes on Floer homology and loop space homology, from: "Morse theoretic methods in nonlinear analysis and in symplectic topology", (P Biran, O Cornea, F Lalonde, editors), NATO Sci. Ser. II Math. Phys. Chem. 217, Springer, Dordrecht (2006) 75-108 MR2276949

[4] A Abbondandolo, M Schwarz, On the Floer homology of cotangent bundles, Comm. Pure Appl. Math. 59 (2006) 254-316 MR2190223

[5] A Abbondandolo, M Schwarz, A smooth pseudo-gradient for the Lagrangian action functional, Adv. Nonlinear Stud. 9 (2009) 597-623 MR2560122

[6] N A Baas, R L Cohen, A Ramírez, The topology of the category of open and closed strings, from: "Recent developments in algebraic topology", (A Ádem, J González, G Pastor, editors), Contemp. Math. 407, Amer. Math. Soc. (2006) 11-26 MR2248970

[7] M Betz, R L Cohen, Graph moduli spaces and cohomology operations, Turkish J. Math. 18 (1994) 23-41 MR1270436

[8] K-c Chang, Infinite-dimensional Morse theory and multiple solution problems, Progress in Nonlinear Differential Equations and their Applications 6, Birkhäuser, Boston (1993) MR1196690

[9] M Chas, D Sullivan, String topology arXiv:math/9911159

[10] M Chas, D Sullivan, Closed string operators in topology leading to Lie bialgebras and higher string algebra, from: "The legacy of Niels Henrik Abel", Springer, Berlin (2004) 771-784 MR2077595

[11] K Cieliebak, T Ekholm, J Latschev, Compactness for holomorphic curves with switching Lagrangian boundary conditions, to appear in J. Symplectic Geom. arXiv: 0903. 2200

[12] K Cieliebak, J Latschev, The role of string topology in symplectic field theory, from: "New perspectives and challenges in symplectic field theory", (M Abreu, F Lalonde, L Polterovich, editors), CRM Proc. Lecture Notes 49, Amer. Math. Soc. (2009) 113-146 MR2555935

[13] R L Cohen, Morse theory, graphs, and string topology, from: "Morse theoretic methods in nonlinear analysis and in symplectic topology", (P Biran, O Cornea, F Lalonde, editors), NATO Sci. Ser. II Math. Phys. Chem. 217, Springer, Dordrecht (2006) 149184 MR2276951 
[14] R L Cohen, K Hess, A A Voronov, String topology and cyclic homology, Advanced Courses in Math. CRM Barcelona, Birkhäuser Verlag, Basel (2006) MR2251006 Lectures from the Summer School held in Almería, September 16-20, 2003

[15] R L Cohen, J D S Jones, A homotopy theoretic realization of string topology, Math. Ann. 324 (2002) 773-798 MR1942249

[16] R L Cohen, J D S Jones, J Yan, The loop homology algebra of spheres and projective spaces, from: "Categorical decomposition techniques in algebraic topology (Isle of Skye, 2001)”, (G Arone, J Hubbuck, R Levi, M Weiss, editors), Progr. Math. 215, Birkhäuser, Basel (2004) 77-92 MR2039760

[17] R L Cohen, J R Klein, D Sullivan, The homotopy invariance of the string topology loop product and string bracket, J. Topol. 1 (2008) 391-408 MR2399136

[18] R L Cohen, M Schwarz, A Morse theoretic description of string topology, from: "New perspectives and challenges in symplectic field theory", (M Abreu, F Lalonde, L Polterovich, editors), CRM Proc. Lecture Notes 49, Amer. Math. Soc. (2009) 147-172 MR2555936

[19] T tom Dieck, K H Kamps, D Puppe, Homotopietheorie, Lecture Notes in Math. 157, Springer, Berlin (1970) MR0407833

[20] J J Duistermaat, On the Morse index in variational calculus, Advances in Math. 21 (1976) 173-195 MR0649277

[21] A Floer, A relative Morse index for the symplectic action, Comm. Pure Appl. Math. 41 (1988) 393-407 MR933228

[22] A Floer, The unregularized gradient flow of the symplectic action, Comm. Pure Appl. Math. 41 (1988) 775-813 MR948771

[23] A Floer, Symplectic fixed points and holomorphic spheres, Comm. Math. Phys. 120 (1989) 575-611 MR987770

[24] A Floer, Witten's complex and infinite-dimensional Morse theory, J. Differential Geom. 30 (1989) 207-221 MR1001276

[25] A Floer, H Hofer, Coherent orientations for periodic orbit problems in symplectic geometry, Math. Z. 212 (1993) 13-38 MR1200162

[26] A Floer, H Hofer, D Salamon, Transversality in elliptic Morse theory for the symplectic action, Duke Math. J. 80 (1995) 251-292 MR1360618

[27] K Fukaya, Morse homotopy, $A^{\infty}$-category, and Floer homologies, from: "Proceedings of GARC Workshop on Geometry and Topology '93”, (H-J Kim, editor), Lecture Notes Ser. 18, Seoul Nat. Univ. (1993) 1-102 MR1270931

[28] K Fukaya, Morse homotopy and its quantization, from: "Geometric topology (Athens, GA, 1993)”, (W H Kazez, editor), AMS/IP Stud. Adv. Math. 2, Amer. Math. Soc. (1997) 409-440 MR1470740

[29] K Fukaya, Y-G Oh, H Ohta, K Ono, Lagrangian intersection Floer theory: anomaly and obstruction. Parts I-II, AMS/IP Studies in Adv. Math. 46, Amer. Math. Soc. (2009) MR2553465 
[30] K Fukaya, K Ono, Arnold conjecture and Gromov-Witten invariant, Topology 38 (1999) 933-1048 MR1688434

[31] H Hofer, D A Salamon, Floer homology and Novikov rings, from: "The Floer memorial volume", (H Hofer, C H Taubes, A Weinstein, E Zehnder, editors), Progr. Math. 133, Birkhäuser, Basel (1995) 483-524 MR1362838

[32] H Hofer, E Zehnder, Symplectic invariants and Hamiltonian dynamics, Birkhäuser Adv. Texts, Birkhäuser Verlag, Basel (1994) MR1306732

[33] L Hörmander, Fourier integral operators. I, Acta Math. 127 (1971) 79-183 MR0388463

[34] S Ivashkovich, V Shevchishin, Reflection principle and J-complex curves with boundary on totally real immersions, Commun. Contemp. Math. 4 (2002) 65-106 MR1890078

[35] S Lang, Fundamentals of differential geometry, Graduate Texts in Math. 191, Springer, New York (1999) MR1666820

[36] G Liu, G Tian, Floer homology and Arnold conjecture, J. Differential Geom. 49 (1998) 1-74 MR1642105

[37] G Liu, G Tian, On the equivalence of multiplicative structures in Floer homology and quantum homology, Acta Math. Sin. (Engl. Ser.) 15 (1999) 53-80 MR1701133

[38] D McDuff, D Salamon, J-holomorphic curves and symplectic topology, Amer. Math. Soc. Coll. Publ. 52, Amer. Math. Soc. (2004) MR2045629

[39] S Piunikhin, D Salamon, M Schwarz, Symplectic Floer-Donaldson theory and quantum cohomology, from: "Contact and symplectic geometry (Cambridge, 1994)", (C B Thomas, editor), Publ. Newton Inst. 8, Cambridge Univ. Press (1996) 171-200 MR1432464

[40] D Quillen, Determinants of Cauchy-Riemann operators on Riemann surfaces, Funktsional. Anal. i Prilozhen. 19 (1985) 37-41, 96 MR783704

[41] A Ramírez, Open-closed string topology via fat graphs arXiv:math/0606512

[42] J Robbin, D Salamon, The Maslov index for paths, Topology 32 (1993) 827-844 MR1241874

[43] J Robbin, D Salamon, The spectral flow and the Maslov index, Bull. London Math. Soc. 27 (1995) 1-33 MR1331677

[44] D A Salamon, J Weber, Floer homology and the heat flow, Geom. Funct. Anal. 16 (2006) 1050-1138 MR2276534

[45] M Schwarz, Morse homology, Progress in Math. 111, Birkhäuser Verlag, Basel (1993) MR1239174

[46] M Schwarz, Cohomology operations from $S^{1}$-cobordisms in Floer homology, $\mathrm{PhD}$ thesis, Swiss Federal Inst. of Techn. Zürich (1995)

[47] P Seidel, A biased view of symplectic cohomology, from: "Current developments in mathematics, 2006”, (D Jenison, B Mazur, T Mrowka, W Schmid, R Stanley, S-T Yau, editors), Int. Press, Somerville, MA (2008) 211-253 MR2459307 
[48] S Smale, An infinite dimensional version of Sard's theorem, Amer. J. Math. 87 (1965) 861-866 MR0185604

[49] D Sullivan, Open and closed string field theory interpreted in classical algebraic topology, from: "Topology, geometry and quantum field theory”, (U Tillmann, editor), London Math. Soc. Lecture Note Ser. 308, Cambridge Univ. Press (2004) 344-357 MR2079379

[50] D Sullivan, String topology background and present state, from: "Current developments in mathematics, 2005”, (D Jenison, B Mazur, T Mrowka, W Schmid, R Stanley, S-T Yau, editors), Int. Press, Somerville, MA (2007) 41-88 MR2459297

[51] C Viterbo, The cup-product on the Thom-Smale-Witten complex, and Floer cohomology, from: "The Floer memorial volume", (H Hofer, C H Taubes, A Weinstein, E Zehnder, editors), Progr. Math. 133, Birkhäuser, Basel (1995) 609-625 MR1362844

[52] C Viterbo, Functors and computations in Floer homology with applications II, Preprint (2003) Available at http://www.math.polytechnique.fr/cmat/viterbo/ FCFH.II.2003.pdf

[53] J Weber, Perturbed closed geodesics are periodic orbits: index and transversality, Math. Z. 241 (2002) 45-82 MR1930985

[54] J Weber, Three approaches towards Floer homology of cotangent bundles, J. Symplectic Geom. 3 (2005) 671-701 MR2235858 Conference on Symplectic Topology

[55] K Wehrheim, C T Woodward, Floer cohomology and geometric composition of Lagrangian correspondences arXiv:0905.1368

[56] K Wehrheim, C T Woodward, Functoriality for Lagrangian correspondences in Floer theory, to appear in Quantum Topol. arXiv:0708.2851

[57] K Wehrheim, C T Woodward, Pseudoholomorphic quilts arXiv:0905.1369

[58] K Wehrheim, C T Woodward, Quilted Floer cohomology, Geom. Topol. 14 (2010) 833-902 MR2602853

Dipartimento di Matematica, Universita di Pisa

Largo Bruno Pontecorvo 5, 56127 Pisa, Italy

Universität Leipzig, Mathematisches Institut

Postfach 1009 20, D-04009 Leipzig, Germany

abbondandolo@dm.unipi.it, mschwarz@math.uni-leipzig.de

http://www.dm.unipi.it/ abbondandolo/,

http://www . math.uni-leipzig.de/ schwarz/

Proposed: Yasha Eliashberg

Seconded: Leonid Polterovich, Ralph Cohen
Received: 6 February 2009

Revised: 6 December 2009 